\title{
Pseudoparticle approach to 1D integrable quantum models
}

\author{
J.M.P. Carmelo ${ }^{\mathrm{a}, \mathrm{b}, \mathrm{c}, *}$, P.D. Sacramento ${ }^{\mathrm{d}, \mathrm{c}}$ \\ a Department of Physics, University of Minho, Campus Gualtar, P-4710-057 Braga, Portugal \\ b Center of Physics of University of Minho and University of Porto, P-4169-007 Oporto, Portugal \\ c Beijing Computational Science Research Center, Beijing 100193, China \\ ' Departamento de Fisica and CeFEMA, Instituto Superior Técnico, Universidade de Lisboa, Av. Rovisco Pais, P-1049-001 Lisboa, Portugal
}

\section{A R T I C L E I N F O}

\section{Article history:}

Accepted 7 June 2018

Available online 19 June 2018

Editor: D.K. Campbell

\section{A B S T R A C T}

Over the last three decades a large number of experimental studies on several quasi onedimensional (1D) metals and quasi 1D Mott-Hubbard insulators have produced evidence for distinct spectral features identified with charge-only and spin-only fractionalized particles. They can be also observed in ultra-cold atomic $1 \mathrm{D}$ optical lattices and quantum wires. 1D exactly solvable models provide nontrivial tests of the approaches for these systems relying on field theories. Different schemes such as the pseudofermion dynamical theory (PDT) and the mobile quantum impurity model (MQIM) have revealed that the 1D correlated models high-energy physics is qualitatively different from that of a lowenergy Tomonaga-Luttinger liquid (TLL). This includes the momentum dependence of the exponents that control the one- and two-particle dynamical correlation functions near their spectra edges and in the vicinity of one-particle singular spectral features.

On the one hand, the low-energy charge-only and spin-only fractionalized particles are usually identified with holons and spinons, respectively. On the other hand, "particlelike" representations in terms of pseudoparticles, related PDT pseudofermions, and MQIM particles are suitable for the description of both the low-energy TLL physics and highenergy spectral and dynamical properties of 1D correlated systems.

The main goal of this review is to revisit the usefulness of pseudoparticle and PDT pseudofermion representations for the study of both static and high-energy spectral and dynamical properties of the 1D Lieb-Liniger Bose gas, spin-1/2 isotropic Heisenberg chain, and 1D Hubbard model. Moreover, the relation between the PDT and the MQIM is clarified. The fractionalized particles and related composite pseudoparticles/pseudofermions emerging within such non-perturbative 1D correlated systems are qualitatively different from the Fermi-liquid quasiparticles. In contrast to the holons and spinons, the relation to the electron creation and annihilation operators of the operators associated with the 1D Hubbard model three fractionalized particles is uniquely defined. The occupancy configurations of such fractionalized particles generate all energy and momentum eigenstates of that model. Both the static and dynamical properties of the three models under review are shown to be controlled at all energy scales by pseudofermion phase shifts associated with only zero-momentum forward scattering. The corresponding microscopic processes are much simpler than those of the underlying particles non-perturbative interactions.

(c) 2018 Elsevier B.V. All rights reserved.

\section{Contents}

1. Introduction...

* Corresponding author at: Department of Physics, University of Minho, Campus Gualtar, P-4710-057 Braga, Portugal.

E-mail address: carmelo@fisica.uminho.pt (J.M.P. Carmelo). 


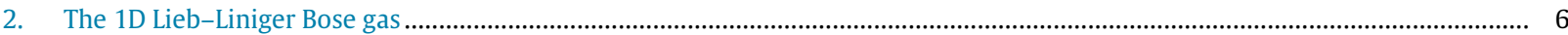

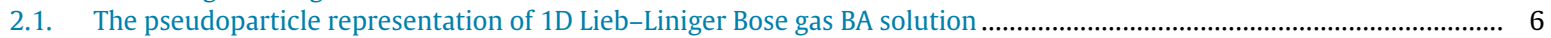

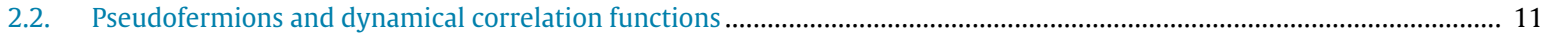

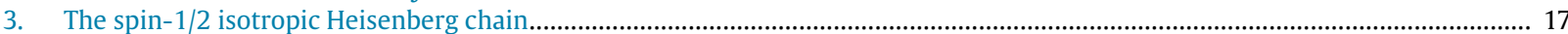

3.1. The model physics: fractional excitations, spin ordering, and magnetism................................................................ 17

3.2. A functional representation of the spin-1/2 XXX chain Bethe-ansatz solution .............................................................. 18

3.3. The $n$-pseudoparticles representation of the spin-1/2 XXX chain Bethe-ansatz roots and its relation to the paired physical spins $1 / 2$

3.4. Relation to the physical spins $1 / 2$ of the holes in the TBA quantum numbers distributions ....... 20

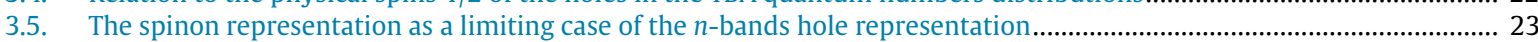

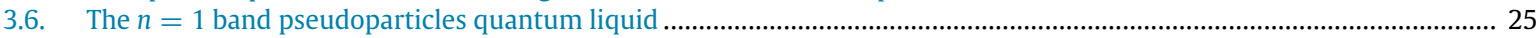

3.7. The spin-1/2 XXX chain longitudinal and transverse dynamical structure factors in the vicinity of their lower thresholds 29

3.8. Outlook of the relation between the model physical spins- $1 / 2$ and the $n$ pseudoparticles $/ n$ pseudofermions and corresponding $n$-band holes.....

4. The 1D Hubbard model: emergent fractionalized particles from rotated electrons

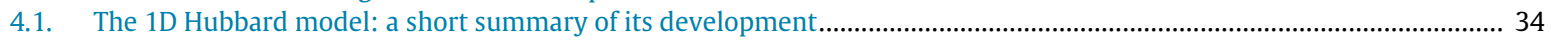

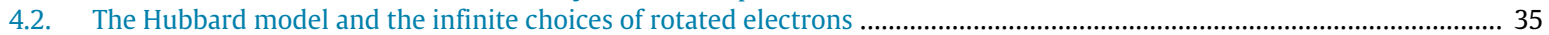

4.3. The Hubbard model BA uniquely defined rotated electrons and corresponding $c$ pseudoparticle, rotated spin, and rotated $\eta$-spin operators.....

4.4. Unpaired and paired spins and $\eta$-spins ...

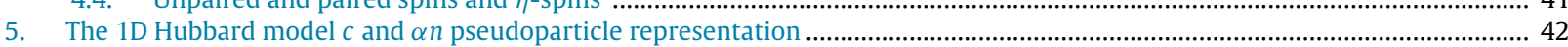

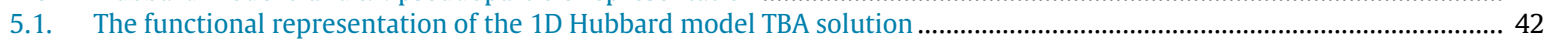

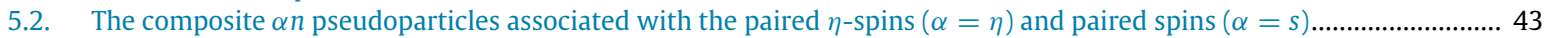

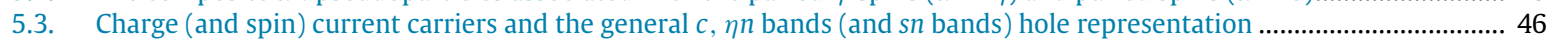

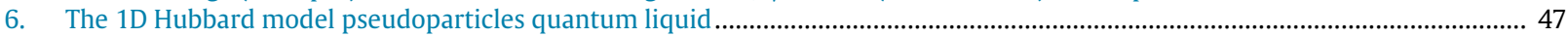

6.1. The $c$ and $\alpha n$ pseudoparticle quantum liquid I: The general energy functional and its energy scales ...............................47

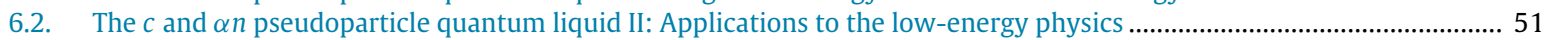

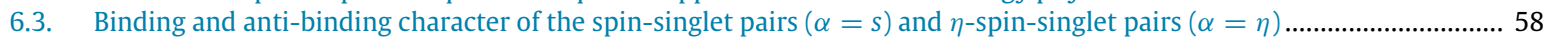

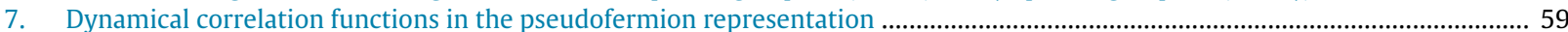

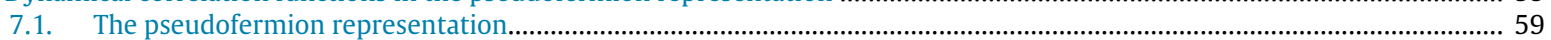

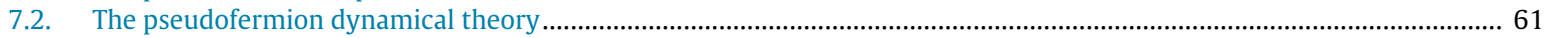

7.3. PDT applications to the high-energy spectral properties of the 1D Hubbard model in a magnetic field ............................66

7.4. Relation between the pseudofermion dynamical theory and the mobile quantum impurity model ...................................6 68

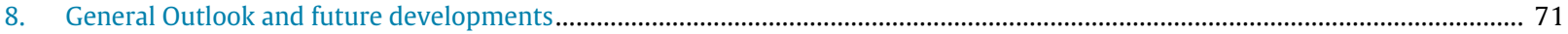

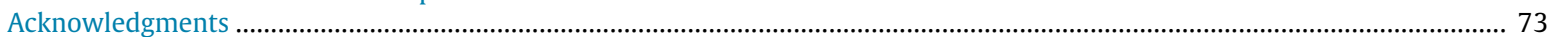

Appendix A. $\quad$ Equality of the Bose gas edge singularities exponents as derived by the PDT and MIM ....................................... 73

Appendix B. Some additional results on the spin-1/2 XXX chain and 1D Hubbard model TBA solutions .................................. 75

Appendix C. Number of state representations of the spin-1/2 XXX chain and 1D Hubbard model symmetries ....................... 77

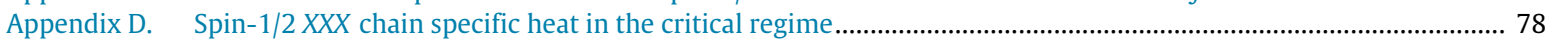

Appendix E. Spin-1/2 XXX chain current expression, susceptibility, and zero-temperature stiffness ………........................... 79

Appendix F. The 1D Hubbard model three fractionalized effective lattices ............................................................................... 81

Appendix G. The 1D Hubbard model s1 pseudoparticle operator representation ................................................................... 81

Appendix H. Integral equations that define the 1D Hubbard model pseudofermion rapidity phase shifts ................................ 82

Appendix I. $\quad \delta S_{\alpha}=\mp n \alpha$-multiplet elementary processes associated with creation and annihilation of one $\alpha n$ pseudoparticle in the spin density $m \rightarrow n_{e}$ limit.....

Appendix J. Derivation of the $\beta=c, s 1$ pseudofermion spectral function within the TL and example of momentum-

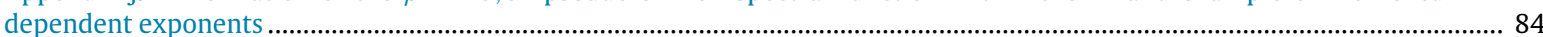

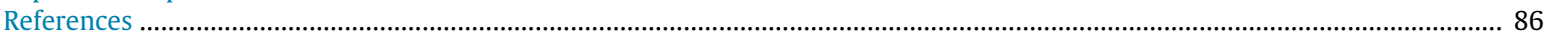

\section{Introduction}

Within quantum physics the isolated electrons are split into smaller components, earning them the designation of a fundamental particle. However this does not necessarily apply when several electrons are brought together. Also the collective behavior of interacting bosonic particles may not be described in terms of that of isolated bosons.

On the one hand, most of the current understanding of many-particle quantum systems involves the concept of a quasiparticle. In three dimensions, starting from free constituents obeying either fermionic or bosonic statistics, one builds a many-particle ground state as either a Fermi sea or a Bose-Einstein condensate. Interactions then adiabatically deform the ground state into a Fermi liquid with well-defined electron-like excitations, or a condensate state with Bogoliubov-like modes, respectively. In both cases, these well-defined excitations are conveniently described as quasiparticles. Moreover, they reveal themselves via sharp lines in dynamical correlation functions, indicative of free-particle-like coherently propagating modes.

On the other hand, one-dimensional (1D) interacting systems are characterized by a breakdown of the basic Fermi liquid quasiparticle picture. Indeed, no quasiparticles occur when the electrons range of motion is restricted to a single 
spatial dimension [1,2]. In a 1D chain correlated electrons rather split into basic fractionalized charge-only and spin-only particles [2-4]. These fractionalized particles can move with different speeds and even in different directions in the 1D many-electron system. Electrons in that system have this ability because they behave like waves. When excited, such waves can split into multiple waves, each carrying different characteristics of the electron. This occurs because collective modes take over. Indeed, applying perturbations does not create single Fermi-liquid quasiparticles. It rather originates an energy continuum of excitations described by exotic fractionalized particles. The latter emerge within 1D many-particle systems. However, they cannot exist independently outside such systems. Moreover, they are not adiabatically connected to free particles. Hence they must be described using a different language.

In electronic systems and spin chains these characteristic fractionalized-particle continua of excitations have been observed [5-8]. Realizations of 1D quantum liquids are numerous. They can take the form of, for example, quasi-1D materials. Over the last three decades, many angle-resolved photoemission spectroscopy (ARPES) studies on several quasi1D metals and quasi-1D Mott-Hubbard insulators have indeed revealed separate charge and spin spectral features. This includes the ARPES spectra of the compound $\mathrm{K}_{0.3} \mathrm{MoO}_{3}$ and other quasi-1D materials [9], quasi-1D organic metals (Bechgaard salts) (TMTSF) ${ }_{2} \mathrm{PF}_{6}$, (TMTSF) ${ }_{2} \mathrm{ClO}_{4}$ (where TMTSF is tetramethyltetraselenafulvalene), and (TMTTF) ${ }_{2} \mathrm{PF}_{6}$ (where TMTTF is tetramethyltetratiafulvalene) [10], quasi-1D metal $\mathrm{Li}_{0.9} \mathrm{Mo}_{6} \mathrm{O}_{17}$ [11,12], quasi-1D organic conductor tetrathiafulvalene tetracyanoquinodimethane (TTF-TCNQ) [13-16], quasi-1D Mott-Hubbard insulators $\mathrm{SrCuO}_{2}$ and $\mathrm{Sr}_{2} \mathrm{CuO}_{3}$ [5,17-20], $\mathrm{NaV}_{2} \mathrm{O}_{5}$ [21], $\mathrm{Na}_{0.96} \mathrm{~V}_{2} \mathrm{O}_{5}$ [22], $\mathrm{SrCuO}_{2}$ and $\mathrm{V}_{6} \mathrm{O}_{13}$ [23], doped quasi-1D Mott-Hubbard insulator $\mathrm{Sr}_{2} \mathrm{CuO}_{3+\delta}$ [24], $1 \mathrm{D}$ metallic surface state on an anisotropic $\mathrm{InSb}(001)$ surface covered with $\mathrm{Bi}$ [25], and metallic 1D line defects in transition dichalcogenides such as $\mathrm{MoSe}_{2}[26]$.

Moreover, similar charge-like and spin-like spectral features were seen as well by electron energy-loss spectroscopic studies on quasi-1D metals and other low-dimensional materials [27]. They were also seen in high-resolution resonant inelastic X-ray scattering on the quasi-1D Mott-Hubbard insulator $\mathrm{Sr}_{2} \mathrm{CuO}_{3}$ [6,7]. The high-resolution resonant inelastic $\mathrm{X}$-ray scattering experiments on $\mathrm{CaCu}_{2} \mathrm{O}_{3}$ reported in Ref. [8] reveal that the orbital hopping in that compound can select different degrees of dimensionality. A spin-orbital fractionalization along the leg direction $x$ through the $x z$ orbital channel was observed as in a 1D system. The mode separation in 1D correlated bosonic and fermionic models and corresponding fractionalized particles are also observed in 1D trapped ultracold atomic gases and ultra-cold atoms on 1D optical lattices [28-38] and quantum wires [39].

The non-perturbative nature of 1D correlated systems prevents their study by conventional perturbative many-body techniques. Nonetheless, some of these systems are exactly solvable by the Bethe ansatz (BA). This method was developed by Bethe in 1931 [40]. He applied it to the spin-1/2 isotropic Heisenberg chain [41]. The BA turned out to be not only useful for that model, but also a very powerful method for a wide range of integrable models. This applies both within and outside the scope of condensed matter physics. The BA provides the exact energy eigenvalues and some thermodynamic quantities. Combined with bosonization [2-4,42-44] or the conformal-invariance associated with the spectra finite-size corrections $[45,46]$, the BA allows the computation of low-energy physics quantities. This has revealed that 1D correlated models share common low-energy properties associated with the universal class of the Tomonaga-Luttinger liquid (TLL) $[44,47,48]$.

One of the main challenges in the study of the 1D correlated systems properties is the calculation of dynamical correlation functions. Indeed, it has been difficult to apply the BA to the derivation of high-energy dynamical correlation functions. (In this review "high energy" means excitation energy values beyond those of the low-energy TLL validity.) The high-energy dynamical correlation functions of some integrable models with spectral gap [49] and spin lattice systems [50] can be studied by the form-factor approach. However, form factors of more complex integrable systems such as the 1D Hubbard model remains an unsolved problem.

The pseudofermion dynamical theory (PDT) has allowed to access that model high-energy dynamical correlation functions beyond the low-energy TLL limit [51]. The theory relies on a suitable pseudofermion representation of the 1D Hubbard model BA solution. Shortly after the PDT was introduced, novel approaches that rely on a mobile quantum impurity model (MQIM) method have been developed to tackle the high-energy physics of both non-integrable and integrable 1D correlated quantum problems [52-57]. The exponents characterizing the dynamical correlation functions singularities have been found by both such schemes to be functions of momenta. They differ significantly from the predictions of the linear TLL theory [13,51-64].

There are several methods and representations for the study of some of the quantum problems reviewed in this paper. The charge-only and spin-only fractionalized particles that emerge in 1D correlated electronic systems [1,2] are within them usually identified with holons and spinons, respectively [65-67]. The conventional holons and spinons have been constructed inherently to be associated with the charge and spin elementary excitations of integrable electronic models, respectively. Moreover, spinons are used to describe the elementary excitations of spin chains. Holons and spinons are defined in terms of the deviation of the charge and spin BA distributions, respectively, from their ground-state value [40,68-79]. This general definition was implemented for the spin excitations of the spin-1/2 XXX chain in Ref. [68]. For the charge and spin excitations of the 1D Hubbard model it was used in Refs. [69,71,74]. A corresponding preliminary example of a spin- $1 / 2$ spinon is the spin-1/2 color spinor introduced in Ref. [76] for the solvable 1D Gross-Neveu model [77]. Its spectrum is associated with one "hole" emerging under a transition from a spin-singlet ground state to an excited energy eigenstate, in a sequence of BA spin quantum numbers. The spin-1/2 spin waves introduced in Ref. [78] for the spin-1/2 XXX chain have a similar definition.

The "particle-like" representations in terms of the pseudoparticles and related pseudofermions discussed in this paper have a uniquely defined yet non-perturbative relation to the models physical particles. (In this review "physical particles" 
refer to the bosons, spins $1 / 2$, and electrons associated with the operators in the models Hamiltonian usual expressions.) The term "pseudoparticle" appeared early in the literature of the Hubbard model [80,81]. More recently it has been used for particles other than those reviewed here [82]. The latter are the pseudoparticles that emerge within 1D integrable models [83,84]. This includes in models with Abelian global $U(1)$ symmetry [61,85], spin- $1 / 2$ chains with a single non-Abelian global SU(2) symmetry [62,86-88], and more complex electronic models [89-105]. In spite of the non-perturbative oneparticle properties of integrable 1D correlated systems, the description of their two-particle static properties is within the pseudoparticle representation very similar to that of a Fermi liquid. Indeed, it is controlled by Landau parameters associated with pseudoparticle residual interactions $f$ functions [89-93].

The PDT pseudofermions are generated from the pseudoparticles under a unitary transformation. It is such that the pseudofermion energy spectrum lacks the pseudoparticle $f$ functions term. As a result, within the thermodynamic limit (TL) the pseudofermions spectrum has no energy interaction terms [64]. Under the transitions from a ground state to one- or two-particle excited states, the pseudofermions scatter off those created or annihilated under the transition. Under such scattering events, the pseudofermions merely acquire a phase shift. Such pseudofermion zero-momentum forward-scattering processes control both the low-energy and high-energy dynamical correlation functions of integrable 1D correlated models $[51,63,64]$. This renders the pseudofermion representation particularly suitable to the study of highenergy dynamical correlation functions.

The pseudoparticle and related pseudofermion representations are here discussed within the constructs of three prominent 1D correlated systems: The 1D Bose gas with two-body repulsive interaction [106-113], the spin-1/2 isotropic Heisenberg chain [40,72,78,114,115], and the repulsive 1D Hubbard model [69-71,73,116-130]. One of the motivations of this review is the physical interest of the pseudoparticle and pseudofermion representations of such low-dimensional correlated systems, which is not purely theoretical. The studies of this review refer to the TL within which the imaginary part of the complex rapidities in the BA equations of models with non-Abelian global symmetries simplify [72,73]. The BA complex rapidities string deviations [131,132] from such ideal strings do not affect in that limit the properties reviewed here.

One of the first applications of the BA to models with Abelian $U(1)$ symmetry was the study of a continuum problem of bosons interacting by a two-body $\delta$-function potential with interaction parameter $c$. Now it is known as 1D Lieb-Liniger Bose gas [106-113]. The model properties depend on the ratio $c / n_{b}$. Here $n_{b}$ is the boson density. As a field theory, this is the repulsive quantum nonlinear Schrödinger model [111]. Although the original quantum problem is given in terms of bosons, the occupancies of model BA distribution have a Pauli-like character, being only zero or one. The model can be simulated in systems of ultra-cold bosonic atomic 1D optical lattices [28,30,31]. Its charge dynamical structure factor can be probed in experimental Bragg spectra of such ultra-cold atoms [33,34]. The static properties of the 1D Lieb-Liniger Bose gas revisited in this review are shown to be naturally described by fermionic-like pseudoparticles with no internal degrees of freedom $[61,85]$. The high-energy one- and two-boson dynamical correlation functions spectral weights are derived within the related pseudofermion representation. They are controlled by pseudofermion phase shifts.

The spin-1/2 isotropic Heisenberg chain [41] is a $1 \mathrm{D}$ model of spins $1 / 2$ with a coupling constant $J$. It was the first quantum system ever to be solved by the BA in 1931 [40]. This spin-chain model remains of great interest due to its underlying richness. In crystals where there is some 1D anisotropy, the model spin chains actually appear and describe the dominant physical behavior [133,134]. Several crystals are known to realize a 1D spin chain described by the spin-1/2 isotropic Heisenberg model. Examples are $\mathrm{KCuF}_{3}, \mathrm{Sr}_{2} \mathrm{CuO}_{3}$ and $\mathrm{CuPzN}$, which have been probed by neutron scattering [135-141]. High-resolution resonant inelastic X-ray scattering revealed the model spectrum in $\mathrm{TiOCl}$ [134] and $\mathrm{La}_{2} \mathrm{CuO}_{4}$ [142]. The spin- $1 / 2 \mathrm{XXX}$ chain can as well be prepared in a 1D ultra-cold atomic trap [32]. The static properties of the spin-1/2 XXX chain discussed in this paper are discussed by use of a representation in terms of spin-neutral composite pseudoparticles [62]. Their constituents are spin-singlet pairs of the model physical spins $1 / 2$. The spin currents of the energy eigenstates are also revisited. It is shown in terms of an exact spin current expression that the elementary currents associated with the model conventional spinons describe the translational degrees of freedom of the model physical spins $1 / 2$ in multiplet configurations. However, such spinons are shown not contain the spin internal degrees of freedom of the latter spins. The model spin dynamical correlation functions are also discussed. They are found to be controlled by the scattering phase shifts of spin-neutral composite pseudofermions.

The general Hubbard model was originally introduced as a toy model to study d-electrons in transition metals [116,117]. It features electrons that can hop between nearest-neighbor lattice sites due to the finite hopping integral $t$. Its sites represent atoms, that are arranged in an ordered, crystalline pattern of well-defined geometry. When two electrons of opposite spin projection are on the same site, they have to pay the energy $U$ due to their mutual repulsion. This introduces additional electronic correlations beyond those of a statistical nature due to the Pauli principle. The model properties depend on the ratio $U / t$. In this paper the parameter $u \equiv U / 4 t$ is often used.

One of the few rigorous results for the Hubbard model on any bipartite lattice refers to its global symmetry. It is well known that on such lattices the model Hamiltonian has two global $S U(2)$ symmetries [143-146]. Consistently, in the early nineties of the past century it was found that for $u \neq 0$ the Hubbard model on a bipartite lattice has at least a $S O(4)=[S U(2) \otimes S U(2)] / Z_{2}$ symmetry. It contains the $\eta$-spin and spin $S U(2)$ symmetries $[144,145]$. More recently it was found in Ref. [147] that for $u \neq 0$ and on any bipartite lattice its global symmetry is actually larger and given by $[S O(4) \otimes U(1)] / Z_{2}=[S U(2) \otimes S U(2) \otimes U(1)] / Z_{2}^{2}$. (This is equivalent to $S O(3) \otimes S O(3) \otimes U(1)$.) The $S U(2)$ and $U(2)=S U(2) \otimes U(1)$ symmetries in the model $[S U(2) \otimes S U(2) \otimes U(1)] / Z_{2}^{2}$ global symmetry refer to the spin and charge degrees of freedom, 
respectively. The charge $U(2)=S U(2) \otimes U(1)$ symmetry includes a $S U(2) \eta$-spin symmetry and a $U(1)$ lattice hidden symmetry beyond $S O(4)$. The latter symmetry is called c-lattice $U(1)$ symmetry in this review. It is indeed associated with the lattice degrees of freedom.

The Hubbard chain is the simplest condensed-matter toy model for the description of the role of correlations in the exotic properties of quasi-1D materials [13-16,148]. It is an important correlated electronic system whose BA solution was first derived by the coordinate BA $[69,70]$. This has followed a similar solution for a related continuous model with repulsive $\delta$-function interaction [149]. Its BA solution was also reached by the inverse-scattering method [120-127]. The non-perturbative relation of the pseudoparticles and related pseudofermions to the electrons involves in the case of the 1D Hubbard model an electron-rotated-electron unitary transformation. It is performed by the exact BA solution. The wellknown $U / t \rightarrow \infty N_{e}$-electron wave functions factorization [4,119,150-163] gives rise under such a unitary transformation to a finite- $U / t$ factorization of the $N_{e}$-rotated-electron wave functions. The corresponding electron-rotated-electron unitary operator is uniquely defined in terms of the matrix elements between the model energy and momentum eigenstates.

The static properties of the 1D Hubbard model discussed in this review are shown to be described by a quantum liquid of several pseudoparticle branches. This includes $c$ pseudoparticles without internal degrees of freedom, spin-neutral composite pseudoparticles, and a third type of $\eta$-spin-neutral composite pseudoparticles. The constituents of the latter are $\eta$-spin-singlet pairs of $\eta$-spin-1/2 fractionalized particles $[51,63,64,105]$. The model one- and two-electron dynamical correlation functions are in this paper reviewed in the suitable pseudofermion representation. Within it such functions spectral weights are controlled by pseudofermion scattering phase shifts [13,51,58,59,63,64].

Concerning new developments, the fact that fractionalized particles are observed in quantum wires [39] renders them candidates for technological applications. Quantum wires are widely used to connect quantum "dots". They may in the future form the basis of quantum computers. Thus the further understanding of the properties of these fractionalized particles may be important for such quantum technologies. It could as well help to develop more complete theories of superconductivity and conduction in low-dimensional condensed-matter systems.

Another new development is the study of the effects of electron finite-range interactions. In the case of 1D, the most physically interesting problems with such interactions are described by non-integrable models. This would be an important new development, since such effects occur in actual low-dimensional systems. For instance, the exponent that controls the suppression of the density of states of most quasi-1D metals [14,15] and metallic 1D line defects in transition dichalcogenides [26] is larger than $1 / 8$. This is a unmistakable signature of electron finite-range interactions [3].

The MQIM was developed to tackle the high-energy physics of both non-integrable and integrable 1D correlated quantum problems [52,53]. In this review the relation between the PDT pseudofermions and the MQIM particles is clarified. The extension of the MQIM to non-integrable 1D correlated electronic systems with long-range interactions is a complex problem. The universality in the vicinity of high-energy one-electron spectral functions singular lines found within the MQIM in Ref. [52] has been preliminarily used to construct a finite-range renormalized model generated from the 1D Hubbard model by a transformation, upon gently turning on suitable potentials [26].

In general, units of both Planck constant $\hbar$ and lattice constant $a$ are used in this paper. In the figures $u$ and $U$ stand for $U / 4 t$ and $U / t$, respectively. If not stated otherwise, the word state refers in this review to an energy and momentum eigenstate. Concerning the general layout of what will be discussed in the following sections, the paper is organized as follows.

In Section 2 the use of a pseudoparticle representation for the 1D Lieb-Liniger Bose gas is addressed. This includes the study of the model static and low-temperature quantities within that representation. A related pseudofermion representation is used to study the high-energy behavior of the one-boson spectral function and charge dynamical structure factor near their spectra edges.

The representation of the spin-1/2 isotropic Heisenberg chain in terms of $n$-pseudoparticles is the topic addressed in Section 3. Here $n=1, \ldots, \infty$ refers to the number of singlet pairs of the model physical spins $1 / 2$ that are bound within a composite $n$-pseudoparticle. The use of such a representation simplifies the study and derivation of the model static and low-temperature quantities. Moreover, a related pseudofermion representation is used in that section to compute the spin longitudinal and transverse dynamical structure factors in the vicinity of their spectra lower thresholds. The relation of the dynamical structure factors peaks to the inelastic neutron scattering experiments on actual spin-chain compounds is discussed. The spinon representation of the model is also addressed. A related extended BA $n$-bands hole representation valid for the model in its full Hilbert space is discussed. Combining the $n$-pseudoparticle and $n$-bands hole representations provides valuable physical information on the processes that control the model spin currents. The relation of both the $n$-pseudoparticles and $n$-band holes to the model physical spins $1 / 2$ is also clarified.

In Section 4 the 1D Hubbard model is introduced. Out of the infinite choices of rotated electrons that follow from its global $[S U(2) \otimes S U(2) \otimes U(1)] / Z_{2}^{2}$ symmetry, those that emerge from the specific electron-rotated-electron unitary transformation performed by the BA solution are considered. The latter rotated electrons degrees of freedom separation is shown to lead to three types of fractionalized particles: The $c$ pseudoparticles without internal degrees of freedom, the rotated spins $1 / 2$, and the rotated $\eta$-spins $1 / 2$.

As in the case of the spin- $1 / 2 X X X$ chain, there emerge within the many-particle system composite sn pseudoparticles that have $n=1, \ldots, \infty$ neutral pairs of rotated spins $1 / 2$ bound within them. This issue is revisited in Section 5 . Additional composite $\eta n$ pseudoparticles that have $n=1, \ldots, \infty$ neutral pairs of rotated $\eta$-spins $1 / 2$ bound within them emerge within the system as well. The relation of the different pseudoparticle types and corresponding $n$-band holes to the rotated electrons and electrons is discussed and clarified. 
In Section 6 the 1D Hubbard model $c$ and $\alpha n$ pseudoparticle quantum liquid is the issue under review. Several physical quantities are derived within the framework of such a quantum liquid by methods that resemble those used in Fermi-liquid theory. The internal configurations of the spin-singlet pairs and $\eta$-spin-singlet pairs are found to have a binding and antibinding character, respectively.

The dynamical correlation functions within the pseudofermion representation is the general issue addressed in Section 7. The PDT version suitable to the 1D Hubbard model is shortly reviewed. Its simplified version applicable to the 1D Lieb-Liniger Bose gas and spin-1/2 XXX chain is also discussed. The effects of varying the spin density on the 1D Hubbard model spectral properties are shortly revisited. Finally, the simpler case of the 1D Bose gas dynamical correlation functions is used to clarify the relation between the PDT and the MQIM approaches.

\section{The 1D Lieb-Liniger Bose gas}

As a first example of application of the pseudoparticle representation of the BA, we consider the 1D Bose gas with twobody repulsive contact interaction. It was introduced in 1963 by Lieb and Liniger [106,107]. The model particles satisfies Bose-Einstein statistics. It is the simplest example of BA solution. Its Hamiltonian describes particles interacting with each other via a two-body potential. The energy and momentum eigenfunctions and eigenvalues can be calculated exactly by the BA. This integrable model helped to shape the understanding of quantum integrability [108-112]. It represents the nonrelativistic limit of several integrable field theories [164-166]. The results of Yang and Yang reported in Ref. [110] were a significant step toward a deeper understanding of the physics of the Lieb-Liniger gas. Indeed, they presented for the first time a grand canonical description of the model in equilibrium.

The model seemed to be only of academic interest until with the sophisticated experimental techniques developed in late XX and XXI st century, it became possible to produce this kind of gas using real bosonic ultra-cold atoms as particles. Hence in addition to being a paradigmatic example of a system of interacting bosons on the continuum, it became as well experimentally relevant for the physics of elongated clouds of cold atoms with contact interactions [34,167-170].

As the temperature is lowered, a uniform gas of bosons in three dimensions will undergo a transition to a Bose-Einstein condensate (BEC). In 1D, low-energy fluctuations prevent long-range order. For trapped gases, the situation changes. Three regimes become possible in $1 \mathrm{D}$ : true condensate, quasi-condensate, and a strongly interacting regime, with BEC limited to extremely small interaction between particles [31].

Trapped 1D gases are now accessible experimentally in all regimes [28,30]. The most challenging to obtain is the strongly interacting case. It can survive without fast decay due to a reduced three-body recombination rate, consequence of fermionization. A natural starting point for the theoretical description of 1D atomic gases in this last regime is indeed provided by bosons with delta-function interaction. In that case the fermionization refers to the Pauli-like zero and one allowed occupancies of the BA quantum numbers. As mentioned above, the charge dynamical structure factor of that 1D Lieb-Liniger Bose gas can be probed in experimental Bragg spectra of ultra-cold atoms on optical lattices [28,33,34].

\subsection{The pseudoparticle representation of $1 D$ Lieb-Liniger Bose gas BA solution}

The Hamiltonian of the 1D Bose gas with two-body repulsive interaction is in units of $\hbar=1$ and bare mass $m=1 / 2$ given by,

$$
\hat{H}=-\sum_{j=1}^{N} \frac{\partial^{2}}{\partial x_{j}^{2}}+2 c \sum_{j^{\prime}>j} \delta\left(x_{j}-x_{j^{\prime}}\right)-\mu N_{b} .
$$

Here and throughout this review, $\delta(x)$ denotes the usual Dirac delta-function distribution, $x_{j}$ is the position of the $j$ th particle, $c>0$ gives the strength of the repulsive interaction, and $\mu$ is the chemical potential. This Hamiltonian describes a set of $N_{b}$ particles with bosonic statistics. All properties of the 1D Lieb-Liniger Bose gas depend on the ratio $c / n_{b}$. Here $n_{b}$ is the particle density $n_{b}=N_{b} / L$. The limit of infinite repulsion, $c \rightarrow \infty$, is often called the Tonks-Girardeau limit [171,172].

The Bethe ansatz method is named after the work by Hans Bethe. Bethe found the energy eigenfunctions and spectrum of the 1D spin-1/2 isotropic Heisenberg model [40]. The 1D Lieb-Liniger Bose gas was the next model solved by coordinate BA more than 30 years later. Since it is the simplest example of BA, the BA is introduced here for that model. We start by considering the case of only two bosons, $N_{b}=2$. The wave function is assumed to be a symmetrized product of plane waves of both bosons,

$$
\begin{aligned}
\psi\left(x_{1}, x_{2}\right) & =\psi_{1}\left(x_{1}, x_{2}\right) \text { for } x_{1}>x_{2} \\
& =\psi_{2}\left(x_{1}, x_{2}\right) \text { for } x_{2}>x_{1} .
\end{aligned}
$$

The functions $\psi_{1}\left(x_{1}, x_{2}\right)$ and $\psi_{2}\left(x_{1}, x_{2}\right)$ are expressed as what is called BA wave functions,

$$
\begin{aligned}
& \psi_{1}\left(x_{1}, x_{2}\right)=A_{12}(I) e^{i\left(k_{1} x_{1}+k_{2} x_{2}\right)}+A_{21}(I) e^{i\left(k_{2} x_{1}+k_{1} x_{2}\right)} \text { for } x_{1}>x_{2} \\
& \psi_{2}\left(x_{1}, x_{2}\right)=A_{12}(I I) e^{i\left(k_{2} x_{1}+k_{1} x_{2}\right)}+A_{21}(I I) e^{i\left(k_{1} x_{1}+k_{2} x_{2}\right)} \text { for } x_{2}>x_{1} .
\end{aligned}
$$

By using Bose statistics, $\psi_{2}\left(x_{2}, x_{1}\right)=\psi_{1}\left(x_{1}, x_{2}\right)$, one finds that the amplitudes in these equations obey the relations,

$$
A_{12}(I)=A_{12}(I I) \equiv A_{12} \text { and } A_{21}(I)=A_{21}(I I) \equiv A_{21} \text {. }
$$


A key feature associated with the BA wave function for this model is that it is continuous at $x_{1}=x_{2}$ whereas its derivative is discontinuous. The second derivative discontinuity reads,

$$
\left.\left(-\frac{\partial^{2}}{\partial x_{1}^{2}}-\frac{\partial^{2}}{\partial x_{2}^{2}}\right) \psi\right|_{x_{2}<x_{1}}-\left.\left(-\frac{\partial^{2}}{\partial x_{1}^{2}}-\frac{\partial^{2}}{\partial x_{2}^{2}}\right) \psi\right|_{x_{1}<x_{2}}=\left.2 c \psi\right|_{x_{1}=x_{2}} .
$$

It then follows from the corresponding continuity and discontinuity conditions that the amplitudes $A_{12}$ and $A_{21}$ satisfy the following relation,

$$
\frac{A_{12}}{A_{21}}=\frac{k_{1}-k_{2}+i c}{k_{1}-k_{2}-i c} \equiv S_{12} .
$$

$S_{12}$ is here the scattering amplitude. It corresponds to an interchange of the regions $x_{1}<x_{2}$ and $x_{1}>x_{2}$ that actually refers to an interchange of the particles.

The system is considered to be on a ring with length $L$. The wave function must then satisfy the boundary condition,

$$
\psi\left(x_{1}+L, x_{2}\right)=\psi\left(x_{1}, x_{2}\right) \text { for } x_{1}<x_{2} .
$$

Its use leads to,

$$
e^{i k_{j} L}=\prod_{l \neq j} S_{l j}=\prod_{l \neq j} \frac{k_{j}-k_{l}+i c}{k_{j}-k_{l}-i c} \text { for } j=1, \ldots, N_{b} .
$$

This is the BA equation that here was derived for $N_{b}=2$. However, it turns out that it is valid for any $N_{b}$. The corresponding $N_{b}$-boson BA wave function is then found to be given by,

$$
\psi\left(x_{1}, \ldots, x_{N_{b}}\right)=\sum_{\iota j \in S_{N_{b}}}^{N_{b} !} A_{\iota} e^{i \sum_{j=1}^{N_{b}} k_{l_{j}} x_{j}} .
$$

Here $S_{N_{b}}$ is the permutation group of $N_{b}$ elements, $\iota$ is one of the elements of $S_{N_{b}}$, and the amplitude reads,

$$
A_{\iota}=(-1)^{\iota} \prod_{j>l}^{N_{b}}\left(k_{l_{j}}-k_{\iota l}-i c \operatorname{sgn}\left\{x_{j}-x_{l}\right\}\right) \text {. }
$$

(Further details on the $N_{b}$-boson BA solution can be found in Ref. [112].)

By taking the logarithm of Eq. (2), one arrives to the following form for the BA equations of the 1D Lieb-Liniger Bose gas [85,106,107],

$$
q_{j}=k_{j}+\frac{2}{L} \sum_{l=1}^{N} \arctan \left(\frac{k_{j}-k_{l}}{c}\right) \text { where } q_{j}=\frac{2 \pi}{L} I_{j} \text { and } j=1, \ldots, \infty .
$$

The $l=1, \ldots, N_{b}$ summation in this equation runs over the subset of occupied $q_{l}$ quantum numbers out of the full $j=1, \ldots, \infty$ set $\left\{q_{j}\right\}$ and corresponding subset of rapidities $k_{l}$. The different occupancy configurations of the related $j=1, \ldots, \infty$ quantum numbers $I_{j}$ in this equation (defined modulo $L$ ) generate all the model energy and momentum eigenstates. Such numbers are successive integers or half-odd integers according to the boundary conditions,

$$
\begin{aligned}
I_{j} & =0, \pm 1, \pm 2, \ldots, \pm \infty \text { for } N_{b} \text { odd } \\
& = \pm \frac{1}{2}, \pm \frac{3}{2}, \ldots, \pm \infty \text { for } N_{b} \text { even. }
\end{aligned}
$$

As confirmed in the following, the corresponding quantities $q_{j}=(2 \pi / L) I_{j}$ in Eq. (3) play the role of discrete momentum values. The quantum numbers, Eq. (4), are successive integers or half-odd integers. The discrete momentum values $q_{j}=$ $(2 \pi / L) I_{j}$ have though the usual spacing for any energy and momentum eigenstate,

$$
q_{j+1}-q_{j}=\frac{2 \pi}{L} \text { where } j=1, \ldots, \infty \text {. }
$$

The set of $j=1, \ldots, \infty$ quantities $k_{j}$ on the right-hand side of Eq. (3) are the BA real momentum rapidities mentioned above. The term rapidity was first used by L. Hulthén in 1938 [173] to parametrize the roots of the spin-1/2 isotropic Heisenberg chain. For it and other models whose BA solution is more complex than that of the present 1D Bose gas, rapidity is actually an analogy with relativistic kinematics, in the relative motion problem. In that case a velocity may become nonadditive due to a transformation, as the Lorentz transformation. One then introduces related suitable alternative additive parameters, called rapidities.

The actual quantum numbers whose occupancy configurations generate the model energy eigenstates are not the momentum rapidities. They are rather the set of numbers $\left\{q_{j}\right\}=\left\{(2 \pi / L) I_{j}\right\}$. The corresponding set $j=1, \ldots, \infty$ of numbers $\left\{I_{j}\right\}$ is given in Eq. (4). Each energy eigenstate is defined by the subset $l=1, \ldots, N_{b}$ of numbers $\left\{q_{l}\right\}=\left\{(2 \pi / L) I_{l}\right\}$ that are occupied. 
The BA equations, Eq. (3), define for each specific subset $l=1, \ldots, N_{b}$ of occupied quantum numbers $\left\{q_{l}\right\}=\left\{(2 \pi / L) I_{l}\right\}$ the corresponding related set $j=1, \ldots, \infty$ of real momentum rapidities $k_{j}$ for the energy eigenstate under consideration. The physical meaning of these equations is thus directly related to that of such momentum rapidities, $k_{j}=k\left(q_{j}\right)$. For instance, the energy eigenvalues depend on the occupancy configurations of the subset $l=1, \ldots, N_{b}$ of quantum numbers $\left\{q_{l}\right\}$ and thus $\left\{I_{l}\right\}$ through them.

The momentum rapidities, $k_{j}=k\left(q_{j}\right)$, and thus the BA equations, Eq. (3), that define them, contain important physical information beyond the mere dependence of the energy eigenvalues on the subset $l=1, \ldots, N_{b}$ of occupied numbers $\left\{q_{l}\right\}$. For instance and as further discussed below in Section 2.2, one finds from straightforward manipulations of these equations that the momentum rapidities $k_{j}=k\left(q_{j}\right)$ of energy eigenstates whose subset $l=1, \ldots, N_{b}$ of occupied numbers $\left\{q_{l}\right\}=\left\{(2 \pi / L) I_{l}\right\}$ differs from that of a ground state in only the occupancies of $N_{\mathrm{ex}} \ll N_{b}$ such numbers have a simple form. For such states, $N_{\mathrm{ex}} / N_{b} \rightarrow 0$ for $N_{b} \rightarrow \infty$. Indeed, their momentum rapidities are within the TL expressed in terms of the corresponding ground-state momentum rapidity $k_{0}\left(q_{j}\right)$ as $k\left(q_{j}\right)=k_{0}\left(q_{j}+2 \pi \Phi\left(q_{j}\right) / L\right)$. Here $2 \pi \Phi\left(q_{j}\right)=\sum_{l=1}^{N e x}\left(\alpha_{l}\right) 2 \pi \Phi\left(q_{j}, q_{l}\right)$ is a dressed phase shift. The quantity $\alpha_{l}$ reads $\alpha_{l}=-1$ and $\alpha_{l}=+1$ when the occupancy of the number $q_{l}$ changes relative to the ground state from occupied to unoccupied and vice versa, respectively. Furthermore, the important two-parameter dressed phase shifts $2 \pi \Phi\left(q_{j}, q_{l}\right)$ are the solution of coupled equations that are directly extracted from the BA equations, Eq. (3). form,

Here we use the functional representation of Ref. [85]. Within it, the energy and momentum eigenvalues are of the general

$$
E=\sum_{l=1}^{N_{b}} k_{l}^{2}-\mu N_{b}=\sum_{j=1}^{\infty} N\left(q_{j}\right) k^{2}\left(q_{j}\right)-\mu N_{b} \text { and } P=\sum_{l=1}^{N_{b}} k_{l}=\sum_{j=1}^{\infty} N\left(q_{j}\right) k\left(q_{j}\right)=\sum_{j=1}^{\infty} N\left(q_{j}\right) q_{j},
$$

respectively. The distribution function $N\left(q_{j}\right)$ appearing here is such that $N\left(q_{j}\right)=1$ and $N\left(q_{j}\right)=0$ for occupied and unoccupied $q_{j}$ values, respectively. We associate one pseudoparticle with each of the $N_{b}$ occupied momentum values $q_{j}$ of such a distribution. That discrete momentum variable, Eq. (3), has the range $q_{j} \in[-\infty, \infty]$. The equality between the two last terms of the momentum expression in Eq. (6) is confirmed by suitable manipulations of the BA equation, Eq. (3).

Each energy eigenstate has specific values for the distribution function $N\left(q_{j}\right)$. The $j=1, \ldots, \infty$ rapidity momentum values $k_{j}$ on the right-hand side of Eq. (3) are for each state functions of the $j=1, \ldots, \infty$ momentum values $q_{j}$, Eq. (3), $k_{j}=k\left(q_{j}\right)$. The pseudoparticles of the 1D Lieb-Liniger Bose gas have no internal structure. Within an alternative "holon" representation, the holons would be associated with the unoccupied momentum values $q_{j}$. However, for the present model there is no advantage in considering such a representation.

For a ground state with $N_{b}$ bosons, the pseudoparticle momentum distribution function is of the form [85],

$$
N^{0}\left(q_{j}\right)=\theta\left(q_{F}-\left|q_{j}\right|\right) \text { where } q_{F}=\pi\left(n_{b}-\frac{1}{L}\right) \approx \pi n_{b} \text { and } q_{F}^{\iota}=\iota \frac{2 \pi}{L} N_{b, \iota} \text { for } \iota= \pm .
$$

The distribution $\theta(x)$ reads in this review $\theta(x)=1$ for $x>0$ and $\theta(x)=0$ for $x \leq 0$. Moreover, $q_{F}$ is in Eq. (7) the pseudoparticle Fermi momentum. Within the TL, one can use the number $N_{b, \iota}$ of $\iota=-1$ left and $\iota=+1$ right pseudoparticles, with the ground-state left and right Fermi momentum value $q_{F}^{l}$ as given in Eq. (7). (In that limit, the $N_{b}$-odd occupancy of the $q_{j}=0$ momentum refers to a $1 / L$ correction to the numbers of left and right pseudoparticles that can be ignored.)

The excited states momentum distribution functions can be written as,

$$
N\left(q_{j}\right)=N^{0}\left(q_{j}\right)+\delta N\left(q_{j}\right) .
$$

Here $N^{0}\left(q_{j}\right)$ is the ground state momentum distribution function, Eq. (7), and $\delta N\left(q_{j}\right)$ is the corresponding momentum distribution function deviation. Under transitions from the $N_{b}$-boson ground state to $N_{b}+\delta N_{b}$-boson excited states for which $\delta N_{b}$ is an even number, the deviations, Eq. (8), can have the values $0,+1$, and -1 . Under transitions to $N_{b}+\delta N_{b}$-boson excited states for which $\delta N_{b}$ is an odd number, one must account for the overall $\pm \pi / L$ shifts in the set of $j=1, \ldots, \infty$ discrete momentum values $q_{j}$, Eq. (3). This effect is due to the corresponding quantum numbers $I_{j}$ being successive half-odd integers for $N_{b}$ even and integers for $N_{b}$ odd, as reported in Eq. (4). We denote the corresponding overall shifts by $(2 \pi / L) \Phi^{0}$ where the parameter $\Phi^{0}$ reads,

$$
\Phi^{0}=0 \text { for } \delta N_{b} \text { even and } \Phi^{0}= \pm \frac{1}{2} \text { for } \delta N_{b} \text { odd . }
$$

For $\delta N_{b}$ odd one must then add the term $\pm 1 / 2$ to the above $\delta N\left(q_{j}\right)$ values $0,+1$, or -1 .

In spite of the bosonic nature of the present quantum problem, the BA quantum numbers, Eq. (4), and corresponding discrete momentum values, Eq. (3), have Pauli-like occupancies zero and one, respectively. Due to such a fermionization, the pseudoparticles that carry these momentum values are not simple "dressed bosons". Indeed, addition or removal of one boson of vanishing energy to and from the ground state involves two pseudoparticle excitations that cannot be decomposed: (a) addition or removal, respectively, of one pseudoparticle of momentum $\pm q_{F} \approx \pm \pi n_{b}$ at the ground-state Fermi points and (b) a collective excitation of all remaining pseudoparticles, each contributing with a small fraction $\mp \pi / L$ or $\pm \pi / L$ to the momentum of the added or removed boson, respectively. This latter excitation results from the pseudoparticle discrete momentum values, Eq. (3), shake up. This effect is associated with the transition between the two set of quantum number 
values in Eq. (4) upon changing $N_{b}$ by one. Although in the present TL each fraction $\mp \pi / L$ or $\pm \pi / L$ is vanishing small, if we multiply by the number of $\approx N_{b}$ pseudoparticles of the Fermi sea this gives $\mp q_{F}$ or $\pm q_{F}$, respectively. Such a collective excitation occurs upon any transition between two arbitrary states differing in the number of bosons by an odd number.

It is useful to classify the general deviations $\delta N\left(q_{j}\right)$ into deviations $\delta N^{F}\left(q_{j}\right)$ and $\delta N^{N F}\left(q_{j}\right)$ for which the quantity $q_{F}-\left|q_{j}\right|$ vanishes and remains finite within the TL, respectively. A particle subspace (PS) is spanned by a ground state and the set of all states generated from it by a finite number of pseudoparticle processes such that $\sum_{j=1}^{\infty}\left|\delta N^{N F}\left(q_{j}\right)\right| / L \rightarrow 0$ as $L \rightarrow \infty$. The transitions from the ground state to its PS excited states include the shake-up effects. Those are associated with the overall momentum shifts $(2 \pi / L) \Phi^{0}$ of all the system pseudoparticles.

In the following we consider the pseudoparticle quantum liquid described by the present model Hamiltonian in the PS of a ground state with arbitrary value of the boson density $n_{b}$. The excitation energy $\delta E=E_{n}-E_{0}$ of the corresponding PS states is up to $\mathcal{O}(1 / L)$ given by [85],

$$
\delta E=\sum_{j=1}^{\infty} \varepsilon\left(q_{j}\right) \delta N\left(q_{j}\right)+\frac{1}{L} \sum_{j=1}^{\infty} \sum_{j^{\prime}=1}^{\infty} \frac{1}{2} f\left(q_{j}, q_{j^{\prime}}\right) \delta N\left(q_{j}\right) \delta N\left(q_{j^{\prime}}\right) .
$$

The only restriction to the applicability of the pseudoparticle energy functional, Eq. (10), is that associated with the PS definition, i.e. $\lim _{L \rightarrow \infty} \sum_{j=1}^{L}\left|\delta N^{N F}\left(q_{j}\right)\right| / L \rightarrow 0$.

The pseudoparticle dispersion $\varepsilon\left(q_{j}\right)$ in the term of first order in the deviations is plotted in Fig. 1 as a function of the momentum $q_{j}$ for several densities $n_{b}$ and interaction $c$ values. It is of the form,

$$
\varepsilon\left(q_{j}\right)=\varepsilon^{0}\left(q_{j}\right)-\mu \text { where } \varepsilon^{0}\left(q_{j}\right)=\left(k_{0}\left(q_{j}\right)\right)^{2}+2 \int_{-Q}^{Q} d k k \bar{\Phi}\left(k, k_{0}\left(q_{j}\right)\right) \text { and } Q= \pm k_{0}\left( \pm q_{F}\right) .
$$

Here $\pm Q$ are the Fermi rapidity momenta, the ground-state momentum rapidity function $k_{0}\left(q_{j}\right)$ is the solution of the BA equation, Eq. (3), for the ground-state distribution, Eq. (7), and $\Phi\left(q_{j}, q_{j^{\prime}}\right)$ is a dressed phase shift in units of $2 \pi$. Its physical meaning is clarified below in Section 2.2. It can be written as,

$$
\Phi\left(q_{j}, q_{j^{\prime}}\right)=\bar{\Phi}\left(k_{0}\left(q_{j}\right), k_{0}\left(q_{j^{\prime}}\right)\right) .
$$

The related momentum-rapidity phase shift $\bar{\Phi}\left(k, k^{\prime}\right)$ in units of $2 \pi$ appearing here obeys the integral equation,

$$
\bar{\Phi}\left(k, k^{\prime}\right)=-\frac{1}{\pi} \arctan \left(\frac{k-k^{\prime}}{c}\right)+\frac{1}{\pi c} \int_{-Q}^{Q} d k^{\prime \prime} \frac{\bar{\Phi}\left(k^{\prime \prime}, k^{\prime}\right)}{1+\left(\frac{k-k^{\prime \prime}}{c}\right)^{2}} .
$$

The chemical potential $\mu$ on the right-hand side of Eq. (11) and the energy bandwidth of the ground-state occupied pseudoparticle sea can be written as,

$$
\begin{aligned}
\mu & =\varepsilon^{0}\left(q_{F}\right)=Q^{2}+2 \int_{-Q}^{Q} d k k \bar{\Phi}(k, Q) \text { and } \\
W_{F} & =\varepsilon^{0}\left(q_{F}\right)-\varepsilon^{0}(0)=Q^{2}+2 \int_{-Q}^{Q} d k k(\bar{\Phi}(k, Q)-\bar{\Phi}(k, 0)),
\end{aligned}
$$

respectively. Hence $\varepsilon\left( \pm q_{F}\right)=0$.

The $f$ functions in the term of second order in the momentum distribution function deviations on the right-hand side of Eq. (10) are given by,

$$
f\left(q_{j}, q_{j^{\prime}}\right)=v\left(q_{j}\right) 2 \pi \Phi\left(q_{j}, q_{j^{\prime}}\right)+v\left(q_{j^{\prime}}\right) 2 \pi \Phi\left(q_{j^{\prime}}, q_{j}\right)+\frac{v}{2 \pi} \sum_{\imath= \pm} 2 \pi \Phi\left(\iota q_{F}, q_{j}\right) 2 \pi \Phi\left(\iota q_{F}, q_{j^{\prime}}\right),
$$

where $f\left(q, q^{\prime}\right)=f\left(-q,-q^{\prime}\right)$. The pseudoparticle group velocities in this expression read $v\left(q_{j}\right)=\left.v(q)\right|_{q=q_{j}}$ where,

$$
v(q)=\frac{d \varepsilon(q)}{d q} \text { and } v=v\left(q_{F}\right)
$$

and $v=v\left(q_{F}\right)$ is the (pseudoparticle) Fermi velocity.

As the form of the expressions in Eqs. (10)-(16) indicates, for the model in a PS the present representation refers to a quantum liquid whose pseudoparticles have residual zero-momentum forward-scattering interactions. Those are associated with the energy terms of second order in the deviations in the general energy spectrum, Eq. (10). The difference relative to the zero-momentum forward-scattering interactions of Fermi-liquid quasiparticles is that the latter is valid only in the limit of vanishing excitation energy. Indeed, due to the model integrability, the pseudoparticles zero-momentum forward-scattering interactions rather refer to all energy scales. Hence the pseudoparticle lifetime is infinite. Furthermore, the pseudoparticle occupancy configurations generate all energy and momentum eigenstates from the boson vacuum. On the contrary, in a Fermi liquid the states generated by quasiparticle occupancy configurations are energy eigenstates only in the limit of vanishing excitation energy. 

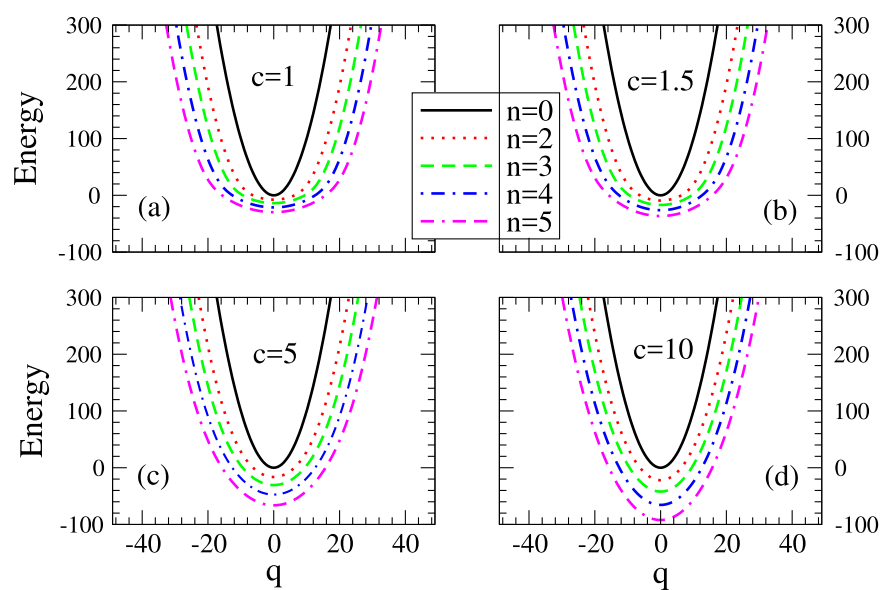

Fig. 1. The pseudoparticle energy dispersion $\varepsilon(q)$, Eq. (11), plotted as a function of the continuous momentum $q$ associated in the TL to $q_{j}$ such that $q_{j+1}-q_{j}=2 \pi / L$ for several densities $n_{b}$ and interaction $c$ values. (In the figures $n_{b}$ is denoted by $n$.)

Within two-boson excitations, the functions $f\left(q_{j}, q_{j^{\prime}}\right)$ play a role similar to that of the $f$ functions in Fermi-liquid theory. The following "renormalized" Fermi velocities determine the low-energy expressions of several physical quantities [85],

$$
v^{i}=v+\frac{1}{2 \pi} \sum_{\iota= \pm}(\iota)^{i} f\left(q_{F}, \iota q_{F}\right)=v\left(\xi^{i}\right)^{2} \text { where } i=0,1 .
$$

The $f$ functions, Eq. (15), in Eq. (17) involve the dressed phase shifts $2 \pi \Phi\left(q_{j}, q_{j^{\prime}}\right)$, Eq. (12). Their connection to the BA equation, Eq. (13), occurs through the momentum rapidity function $k_{j}=k\left(q_{j}\right)$ of PS excited states with general distributions $N\left(q_{j}\right)=N^{0}\left(q_{j}\right)+\delta N\left(q_{j}\right)$, Eq. (8). Their use in the BA equations leads to solutions of the form $k\left(q_{j}\right)=$ $k_{0}\left(q_{j}+[1 / L] \sum_{j^{\prime}} \delta N\left(q_{j^{\prime}}\right) 2 \pi \Phi\left(q_{j}, q_{j^{\prime}}\right)\right)$. The quantity $\Phi\left(q_{j}, q_{j^{\prime}}\right)$ appearing here is related to the rapidity phase shift $\bar{\Phi}\left(k, k^{\prime}\right)$, Eq. (12). The latter obeys the integral equation, Eq. (3). It emerges directly from suitable manipulations of the BA equation.

The $i=0$ and $i=1$ quantities $v^{i} / v=1+(1 / 2 \pi v) \sum_{l= \pm}(\iota)^{i} f\left(q_{F}, \iota q_{F}\right)$ play the role of symmetric charge and antisymmetric current Landau parameters, respectively. The related parameters $\xi^{0}$ and $\xi^{1}$ in Eq. (17) are the following simple symmetric and antisymmetric combinations, respectively, of dressed phase shifts in units of $2 \pi$ at the Fermi points,

$$
\xi^{i}=1+\Phi\left(q_{F}, q_{F}\right)+(-1)^{i} \Phi\left(q_{F},-q_{F}\right) \text { where } i=0,1 .
$$

(Here in $\Phi\left(q_{F}, q_{F}\right)$ and in other phase shifts of this review whose two momenta are the same, $\left(q_{F}, q_{F}\right)$ refers to the TL in which $\left(q_{F}, q_{F} \pm 2 \pi / L\right)$ is for simplicity written as $\left(q_{F}, q_{F}\right)$.) Manipulations of Eqs. (12), (13), and (18) reveal that such parameters are related as $\xi^{1}=1 / \xi^{0}$ and that $\xi^{1}=\xi^{1}(Q)$. Here $\xi^{1}(k)$ is the solution of the integral equation $\xi^{1}(k)=$ $1+(c / \pi) \int_{-O}^{Q} d k^{\prime} \xi^{1}\left(k^{\prime}\right) /\left[c^{2}+\left(k-k^{\prime}\right)^{2}\right]$ where $\pm Q=k_{0}\left( \pm q_{F}\right)$ and $q_{F}=\pi n_{b}$. Its solution is mathematically simplest in the $n_{b} / c \ll 1$ and $n_{b} / c \gg 1$ limits. This leads to the following limiting expressions, which, as all the model quantities, depend only on the ratio $n_{b} / c$,

$$
\xi^{1}=1 / \xi^{0} \approx 1+\frac{2 n_{b}}{c} \text { for } n_{b} / c \ll 1 \text { and } \xi^{1}=1 / \xi^{0} \approx \sqrt{\pi \sqrt{\frac{n_{b}}{c}}}=\pi^{1 / 2}\left(\frac{n_{b}}{c}\right)^{1 / 4} \text { for } n_{b} / c \gg 1 .
$$

The related "renormalized" velocities $v_{0}$ and $v_{1}$, Eq. (17), and the Fermi velocity $v=v\left(q_{F}\right)$, Eq. (16), are given by the following expressions and obey the following relations,

$$
v_{0}=\frac{v^{2}}{2 \pi n_{b}} \text { and } v_{1}=2 \pi n_{b} \text { where } v=2 \pi n_{b}\left(\xi^{0}\right)^{2}=\frac{2 \pi n_{b}}{\left(\xi^{1}\right)^{2}} .
$$

The velocities $v, v_{0}$, and $v_{1}$ are plotted in Fig. 2 as a function of the density $n_{b}$ for $c=1$.

The usual TLL parameter $K_{0}$ can be written as $K_{0}=\left(\xi^{1}\right)^{2}=\left(1 / \xi^{0}\right)^{2}$. This is consistent with at low energy the pseudoparticles referring to a representation of the universal TLL. Its results can be reached by other approaches $[2,57]$, as for instance conformal field theory [174-176]. When, as in Eq. (17), the two momenta of the $f$ functions, Eq. (15), are at the Fermi points, they read $f\left(q_{F}, \pm q_{F}\right)=\pi\left[v^{0}-v \pm\left(v^{1}-v\right)\right]$. This signals the emergence of the low-energy TLL physics.

The system compressibility is controlled by excited states. Their momentum distribution function deviations are given by [85],

$$
\delta N(q)=\delta\left(q_{F}-|q|\right) \delta q_{F} .
$$



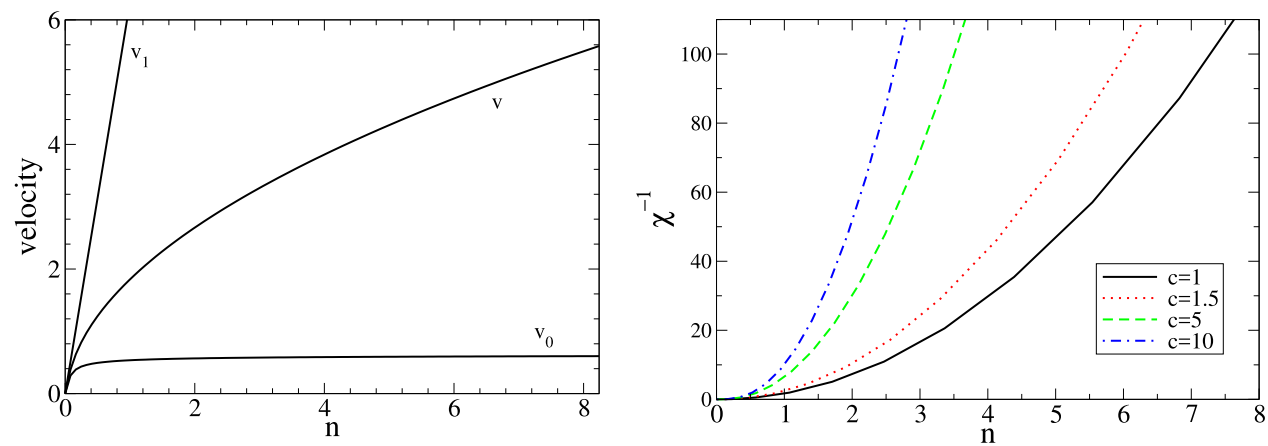

Fig. 2. The group velocity and the two "renormalized" Fermi velocities, Eqs. (17) and (20), (left panel) and the inverse compressibility $\chi^{-1}$, Eq. (22), (right panel) as a function of the density $n_{b}$ for $c=1$ and several interaction $c$ values, respectively. (In the figures $n_{b}$ is denoted by $n$.)

Source: The plots of $v, v_{0}$ and $v_{1}$ (left) were produced using data from Fig. 3 of Ref. [85].

From their use in Eqs. (10) and (14), one readily finds that $\partial \mu\left(n_{b}\right) / \partial n_{b}=-v_{0} \pi$ where $v_{0}$ is defined in Eq. (17). The compressibility is then easily found to read,

$$
\chi=-\frac{1}{n_{b}^{2}} \frac{1}{\partial \mu\left(n_{b}\right) / \partial n_{b}}=\frac{1}{\pi n_{b}^{2}} \frac{1}{v_{0}} .
$$

The inverse compressibility $\chi^{-1}$ is plotted in Fig. 2 as a function of the density $n_{b}$ for several interaction $c$ values.

An expression for the low-temperature entropy is derived by means of simple combinatorial arguments that rely on the allowed occupancies of the discrete momentum values $q_{j}$, Eq. (3), being only zero and one. The result is,

$$
S=-2 \sum_{j=1}^{\infty}\left(N\left(q_{j}\right) \ln \left[N\left(q_{j}\right)\right]+N^{h}\left(q_{j}\right) \ln \left[N^{h}\left(q_{j}\right)\right]\right) .
$$

Here $N^{h}\left(q_{j}\right) \equiv 1-N\left(q_{j}\right)$ and $N\left(q_{j}\right)$ is a Fermi-Dirac distribution. Its temperature-dependent BA energy dispersion $\varepsilon\left(q_{j}\right)$ is obtained in a self-consistent way, as in a Fermi liquid. In the present low-temperature limit we can use in it the $T=0$ energy dispersion, Eq. (11). Although the original particles are bosons, in connection to and due to the BA quantum numbers $I_{j}$ allowed occupancies, Eq. (4), the pseudoparticles obey a Pauli-like occupancy of their momentum values $q_{j}=(2 \pi / L) I_{j}$. Hence their thermal momentum distribution function deviation $\delta N\left(q_{j}\right)$ is given by,

$$
\delta N\left(q_{j}\right)=\frac{1}{1+e^{\varepsilon\left(q_{j}\right) / k_{B} T}}-N^{0}\left(q_{j}\right) .
$$

From the use in the energy functional, Eq. (10), of Eqs. (23) and (24), provided one accounts for that $\varepsilon\left( \pm q_{F}\right)=0$, it is straightforward to obtain the well known TLL low-temperature specific-heat leading order term [174-176],

$$
c_{V}=\frac{L k_{B} \pi}{3 v}\left(k_{B} T\right) .
$$

The charge conductivity real part has the general form $\sigma(\omega)=2 \pi D \delta(\omega)+\sigma_{\text {reg }}(\omega)$. The charge stiffness or Drude weight $D$ characterizes here the response to a static field. $\sigma_{\text {reg }}(\omega)$ describes the absorption of light of frequency $\omega$. Both such quantities can be expressed in terms of the charge current operator [177]. Since the system has translational invariance, for this model that operator commutes with the Hamiltonian. As a result, the real conductivity spectrum has no incoherent part. From the conductivity sum rule one then finds,

$$
\operatorname{Re} \sigma(\omega)=2 \pi D \delta(\omega) \text { where } 2 \pi D=v^{1}=2 \pi n_{b} .
$$

\subsection{Pseudofermions and dynamical correlation functions}

It useful for the study of the dynamical correlation functions of the 1D Lieb-Liniger Bose gas and other integrable models to provide some basic information on dynamical correlation functions of general many-body quantum systems. Time correlation functions of dynamical variables play an important role in the description of such systems. It is well known that transport coefficients and the cross sections for scattering of physical particles are directly related to time correlation functions. The simplest dynamical properties are the linear response to an external perturbation. Most macroscopic measurements are in the linear regime. This is because macroscopic perturbations are very small on the scale of microscopic forces. 
The fluctuation-dissipation theorem states that at zero and finite temperature the linear response of the system to a time-varying perturbation is the same as and is connected to fluctuations, respectively, that naturally occur in statistical equilibrium $[178,179]$. This means that the response of a system in thermodynamic equilibrium to a small applied force is connected to its response to a spontaneous fluctuation. Therefore, the theorem connects the linear response relaxation of a system from a prepared non-equilibrium state to its statistical fluctuation properties in equilibrium. So there are two basic ways to calculate the response: either waiting for the system to fluctuate by itself or applying a perturbation and see what happens. The second is often referred to as the Kubo method. (In the present case of 1D integrable models, see Ref. [180].)

Within quantum field theory, the operators become functions of space and time, $\hat{O}(x, t)$. Consider two operators $\hat{A}(x, t)$ and $\hat{B}(x, t)$ and that the basic dynamical correlation functions,

$$
\chi\left(x, t ; x^{\prime} t^{\prime}\right)=\left\langle\hat{A}(x, t) \hat{B}\left(x^{\prime}, t^{\prime}\right)\right\rangle,
$$

where

$$
\hat{A}(x, t)=e^{i \hat{H} t} \hat{A}(x) e^{-i \hat{H} t} \text { and } \hat{B}(x, t)=e^{i \hat{H} t} \hat{B}(x) e^{-i \hat{H} t},
$$

are written within the Heisenberg representation. Here $\hat{H}$ is the Hamiltonian of the model under consideration and $\langle\ldots\rangle$ stands for the thermal average for finite temperatures $T>0$ and the ground-state expectation value at $T=0$.

The dynamical correlation functions, Eq. (27), are the building blocks of several physically relevant dynamical correlation functions. This includes, for instance, the dynamical correlation functions,

$$
\begin{aligned}
\chi_{\text {ret }}\left(x, t ; x^{\prime} t^{\prime}\right) & =-i \theta\left(t-t^{\prime}\right)\left\langle\left[\hat{A}(x, t) \hat{B}\left(x^{\prime}, t^{\prime}\right) \pm \hat{B}\left(x^{\prime}, t^{\prime}\right) \hat{A}(x, t)\right]\right\rangle \text { and } \\
\chi^{\prime \prime}\left(x, t ; x^{\prime} t^{\prime}\right) & =\left\langle\left[\hat{A}(x, t), \hat{B}\left(x^{\prime}, t^{\prime}\right)\right]\right\rangle,
\end{aligned}
$$

where in the retarded correlation function $\chi_{\text {ret }}\left(x, t ; x^{\prime} t^{\prime}\right)$ expression + and - refers to fermions and bosons, respectively. In most cases of physical interest, the correlation function $\chi^{\prime \prime}\left(x, t ; x^{\prime} t^{\prime}\right)$ plays the role of the quantity that describes absorption or dissipation. The related retarded correlation function $\chi_{\mathrm{ret}}\left(x, t ; x^{\prime} t^{\prime}\right)$ is characterized by the presence of the step function $\theta\left(t-t^{\prime}\right)$. Usually, when thinking about scattering amplitudes, one works with time-ordered (Feynman) correlation functions. Those are relevant for building perturbation theory.

In most cases zero temperature is considered in this review, so that $\langle\ldots\rangle$ means ground-state expectation value $\langle G S|\ldots| G S\rangle$. The systems considered in it exhibit within the TL translational invariance in both space and time. Hence the dynamical correlation functions depend only on the differences $x-x^{\prime}$ and $t-t^{\prime}$, respectively. One then defines the dynamical correlation functions in momentum and frequency space by the Fourier transforms,

$$
\tilde{\chi}(k, \omega)=\int d x \int d t e^{-i(k x-\omega t)} \chi(x, t ; 0,0) .
$$

In the problems under consideration in this review one has that $\hat{A}(x, t)=\hat{O}(x, t)$ and $\hat{B}(x, t)=\hat{O}^{\dagger}(x, t)$ in Eqs. (27) and (29), so that for instance,

$$
\begin{aligned}
\chi_{\text {ret }}\left(x, t ; x^{\prime} t^{\prime}\right) & =-i \theta\left(t-t^{\prime}\right)\left\langle\left[\hat{O}(x, t) \hat{O}^{\dagger}\left(x^{\prime}, t^{\prime}\right) \pm \hat{O}^{\dagger}\left(x^{\prime}, t^{\prime}\right) \hat{O}(x, t)\right]\right\rangle \text { and } \\
\tilde{\chi}_{\text {ret }}(k, \omega) & =\int d x \int d t e^{-i(k x-\omega t)} \chi_{\text {ret }}(x, t ; 0,0) .
\end{aligned}
$$

It is useful to express the dynamical correlation function $\tilde{\chi}_{\text {ret }}(k, \omega)$ in this equation in a Lehmann representation. At zero temperature it reads,

$$
\begin{aligned}
\tilde{\chi}_{\text {ret }}(k, \omega) & =\sum_{v} \frac{|\langle\nu|\hat{O}(k)| G S\rangle|^{2}}{\omega-\left(E_{v}-E_{G S}\right)+i \delta} \text { so that } \\
-\frac{1}{\pi} \operatorname{Im} \tilde{\chi}_{\mathrm{ret}}(k, \omega) & =\sum_{\nu}|\langle\nu|\hat{O}(k)| G S\rangle|^{2} \delta\left(\omega-\left(E_{v}-E_{G S}\right)\right),
\end{aligned}
$$

where $\delta$ is as usual an infinitesimal number, $\sum_{\nu}$ is a sum that runs over energy eigenstates, and,

$$
\hat{O}(k)=\int d x e^{-i k x} \hat{O}(x) .
$$

Correlation functions are important quantities in many branches of physics. For instance, in the framework of the physics of ultracold quantum gases, they provide valuable information about the quantum many-body wave function beyond the simple density profile [113]. The charge dynamical structure factor of a 1D Lieb-Liniger Bose gas is an example of a dynamical correlation function that can be probed in experimental Bragg spectra of ultra-cold atoms on optical lattices [33,34].

There is an alternative fermionic representation for the 1D Lieb-Liniger Bose gas in terms of new entities called pseudofermions. These emerge naturally from the pseudoparticles in a given PS. Such an alternative representation is particularly suitable for the study of the dynamical correlation functions of the integrable models reviewed in this paper. 
Their emergence from the pseudoparticles involves an important property of the momentum rapidity functions $k_{j}=k\left(q_{j}\right)$ of the excited states of any PS. Manipulations of the BA equations, Eq. (3), that involve the use of distributions of form $N\left(q_{j}\right)=N^{0}\left(q_{j}\right)+\delta N\left(q_{j}\right)$, Eq. (8), and the expansion of these equations in such deviations, reveals that the excited-state functions $k_{j}=k\left(q_{j}\right)$ can be exactly expressed in terms of the corresponding ground-state rapidity function $k_{j}^{0}=k_{0}\left(q_{j}\right)$ as follows,

$$
k\left(q_{j}\right)=k_{0}\left(\bar{q}_{j}\right) \text { for } j=1, \ldots, \infty .
$$

Here,

$$
\bar{q}_{j}=q_{j}+\frac{2 \pi \Phi\left(q_{j}\right)}{L} \text { for } j=1, \ldots, \infty \text { where } 2 \pi \Phi\left(q_{j}\right)=\sum_{j^{\prime}=1}^{\infty} \delta N\left(q_{j^{\prime}}\right) 2 \pi \Phi\left(q_{j}, q_{j^{\prime}}\right) .
$$

The pseudofermion representation associates each occupied discrete canonical momentum value $\bar{q}_{j}$, Eq. (33), with one pseudofermion. The quantity $2 \pi \Phi\left(q_{j}\right)$ is below confirmed to be a scattering pseudofermion phase shift. It is such that $2 \pi \Phi\left(q_{j+1}\right)-2 \pi \Phi\left(q_{j}\right)=\mathcal{O}(1 / L)$. Hence the spacing of the discrete canonical momentum values is to leading $\mathcal{O}(1 / L)$ order the usual one,

$$
\bar{q}_{j+1}-\bar{q}_{j}=\frac{2 \pi}{L}+\mathcal{O}\left(1 / L^{2}\right) \text { for } j=1, \ldots, \infty .
$$

However, a similar equality does not hold for the discrete canonical momentum values separation for $l$ values such that $l / N_{b}$ is finite as $N_{b} \rightarrow \infty, \bar{q}_{j+l}-\bar{q}_{j} \neq l(2 \pi / L)$. (It does though for $l=\infty$.)

Since $2 \pi \Phi\left(q_{j+1}\right) / L-2 \pi \Phi\left(q_{j}\right) / L$ is of $\mathcal{O}\left(1 / L^{2}\right)$ order there is no level crossing. By this it is meant that the two sets $\left\{q_{j}\right\}$ of pseudoparticle momenta and $\left\{\bar{q}_{j}\right\}$ of pseudofermion canonical momenta are similarly ordered for $j=1, \ldots, \infty$. For each excited state there is then a pseudoparticle-pseudofermion unitary transformation associated with the one-to-one correspondence between $q_{j}$ and $\bar{q}_{j}=q_{j}+2 \pi \Phi\left(q_{j}\right) / L$. The equality, $\bar{q}_{j}=q_{j}$, holds for the PS ground state, so that for it pseudoparticles and pseudofermions are identical particles.

A key property of the pseudofermion representation, which renders it the most appropriate for the study of dynamical correlation functions, is that upon expressing the energy functional, Eq. (10), in terms of the discrete canonical momentum values $\bar{q}_{j}=\bar{q}\left(q_{j}\right)$, Eq. (33), it simplifies up to $\mathcal{O}(1 / L)$ order to,

$$
\delta E=\sum_{j=1}^{\infty} \varepsilon\left(\bar{q}_{j}\right) \delta \mathcal{N}\left(\bar{q}_{j}\right)
$$

The transformation from $q_{j}$ to $\bar{q}_{j}=q_{j}+2 \pi \Phi\left(q_{j}\right) / L$ absorbs the energy interacting term in Eq. (10). The pseudofermion canonical momentum distribution $\mathcal{N}\left(\bar{q}_{j}\right)$ in Eq. (35) is defined as $\mathcal{N}\left(\bar{q}_{j}\right)=N\left(q_{j}\right)$ where $\bar{q}_{j}=\bar{q}\left(q_{j}\right)$ for $j=1, \ldots, \infty$. The pseudofermion energy dispersion $\varepsilon\left(\bar{q}_{j}\right)$ has exactly the same form as that given in Eq. (11) with the momentum $q_{j}$ replaced by the corresponding canonical momentum, $\bar{q}_{j}=\bar{q}\left(q_{j}\right)$.

In contrast to the equivalent energy functional, Eq. (10), that given in Eq. (35) has no energy interaction terms of secondorder in the deviations $\delta \mathcal{N}\left(\bar{q}_{j}\right)$. Indeed by expanding the canonical momentum $\bar{q}_{j}$ around $q_{j}$ in Eq. (35) and considering all energy contributions up to $\mathcal{O}(1 / L)$ order, one recovers after some lengthy yet straightforward algebra the energy functional, Eq. (151), which includes terms of second order in the deviations $\delta N\left(q_{j}\right)$. Their absence from the corresponding energy spectrum, Eq. (35), is a consequence of the scattering phase shift functional $2 \pi \Phi\left(q_{j}\right)$, Eq. (33), being incorporated in the pseudofermions canonical momentum, Eq. (33).

Physically, the quantity $\pm 2 \pi \Phi\left(q_{j}, q_{j^{\prime}}\right)$, Eq. (12), is the phase shift of a pseudofermion of ground-state momentum $q_{j}$. It is acquired upon it scattering off a pseudofermion created $(+)$ or annihilated $(-)$ at a momentum $q_{j^{\prime}}$ under a transition from the ground state to a PS excited state. The momentum $q_{j^{\prime}}$ refers to that state canonical momentum $\bar{q}_{j^{\prime}}=q_{j^{\prime}}+2 \pi \Phi\left(q_{j^{\prime}}\right) / L$. Hence $2 \pi \Phi\left(q_{j}\right)=\sum_{j^{\prime}=1}^{\infty} \delta N\left(q_{j^{\prime}}\right) 2 \pi \Phi\left(q_{j}, q_{j^{\prime}}\right)$, Eq. (33), is the overall scattering phase shift acquired by such a pseudofermion upon scattering off all pseudofermions created or annihilated under that transition.

The $\iota= \pm$ pseudoparticle Fermi points given in Eq. (7) for a ground state also exist for the excited states that span the corresponding PS. Here we denote the ground-state numbers of right $(\iota=+)$ and left $(\iota=-)$ pseudoparticles by $N_{b, \iota}^{0}$. Hence the Fermi points in Eq. (7) read $q_{F}^{l}=\iota \frac{2 \pi}{L} N_{b, \iota}^{0}$. The PS excited states pseudoparticle Fermi points are of the form $q_{F}^{\iota}=\iota \frac{2 \pi}{L} N_{b, \iota}^{0}+\delta q_{F}^{\iota}$ where $\delta q_{F}^{\iota}=[2 \pi / L] \delta N_{b, \iota}^{F}$ and $\delta N_{b, \iota}=\delta N_{b, \iota}^{F, 0}+\Phi^{0}$ for $\iota= \pm$. The deviation $\delta N_{b, \iota}^{F, 0}$ in this equation refers to the number of pseudoparticles (and pseudofermions) at the $\iota= \pm$ Fermi points. It results from the creation or annihilation pseudoparticle processes and $\Phi^{0}$ is the non-scattering phase shift, Eq. (9), in units of $2 \pi$.

Two quantities that play a key role within the pseudofermion representation of the present model are the $\iota= \pm$ pseudofermion Fermi points deviations,

$$
\delta \bar{q}_{F}^{\iota}=\delta q_{F}^{\iota}+\frac{2 \pi \Phi\left(q_{F}^{\iota}\right)}{L} \text { for } \iota= \pm .
$$

Specifically, the square of such deviations $\delta \bar{q}_{F}^{\iota}$ in units of $2 \pi / L$, which is denoted by $2 \Delta^{\iota} \equiv\left(\delta \bar{q}_{F}^{\iota} /[2 \pi / L]\right)^{2}$, corresponds to two important $\iota= \pm$ fluctuations functionals of the theory. Within the PDT they control the one- and two-particle matrix 
elements quantum overlaps. From manipulations that rely on the form of the phase shift $2 \pi \Phi\left(q_{j}\right)$ in the pseudoparticlemomentum pseudofermion-canonical-momentum transformation $q_{j} \rightarrow q_{j}+2 \pi \Phi\left(q_{j}\right) / L$, Eq. (33), one finds in the TL that,

$$
2 \Delta^{\iota}\left(\left\{q_{j^{\prime}}\right\}\right) \equiv\left(\frac{\delta \bar{q}_{F}^{\iota}}{2 \pi / L}\right)^{2}=\left(\xi^{1} \delta J_{b}^{F}+\iota \frac{\delta N_{b}^{F}}{2 \xi^{1}}+\sum_{j^{\prime}=1}^{\infty} \delta N^{N F}\left(q_{j^{\prime}}\right) \Phi\left(\iota q_{F}, q_{j^{\prime}}\right)\right)^{2} \text { for } \iota= \pm .
$$

The summation $\sum_{j^{\prime}=1}^{\infty} \delta N^{N F}\left(q_{j^{\prime}}\right)$ runs here over a set of finite $j^{\prime}=1, \ldots, N_{b}^{N F}$ values where $N_{b}^{N F}=\sum_{j^{\prime}=1}^{\infty}\left|\delta N^{N F}\left(q_{j^{\prime}}\right)\right|$. Hence $2 \Delta^{l}\left(\left\{q_{j^{\prime}}\right\}\right)$ depends on a corresponding set of finite $q_{1}, \ldots, q_{N^{N F}}$ momentum values.

On the one hand, the deviations $\delta N_{b}^{F}=\sum_{l= \pm} \delta N_{b, \iota}^{F}$ and $\delta J_{b}^{F}=\frac{1}{2} \sum_{\iota= \pm}(\iota) \delta N_{b, l}^{F}$ in Eq. (37) refer to the low-energy part of the excitations. On the other hand, within the TL the high-energy contributions are associated with the deviation $\delta N^{N F}\left(q_{j^{\prime}}\right)$ as defined above. The pseudofermion creation or annihilation at and in the vicinity of the Fermi points is rather accounted for by the deviations $\delta N_{b}^{F}$ and $\delta J_{b}^{F}$.

For low-energy PS excited states for which $\delta N^{N F}\left(q_{j^{\prime}}\right)=0$ for all $q_{j^{\prime}}$ values away from the $\iota= \pm$ pseudofermion Fermi points, the fluctuation functionals, Eq. (37), become the $\iota= \pm$ fields conformal dimensions of a conformal field theory,

$$
2 \Delta_{0}^{\iota} \equiv\left(\frac{\delta \bar{q}_{F}^{\iota}}{2 \pi / L}\right)^{2}=\left(\xi^{1} \delta J^{F}+\iota \frac{\delta N^{F}}{2 \xi^{1}}\right)^{2} \text { for } \iota= \pm .
$$

In the low-energy limit the model can be mapped into a conformal field theory [174-176]. As given in Eq. (18), within the pseudofermion representation the low-energy parameter $\xi^{1}=1 / \xi^{0}$ naturally emerges from the pseudofermion phase shifts at the Fermi points. $\xi^{1}=1 / \xi^{0}$ is actually the dressed charge of the conformal field theory [174-176]. Furthermore, since the usual low-energy TLL parameter $K_{0}[2,57]$ merely reads $K_{0}=\left(\xi^{1}\right)^{2}$.

As shortly reported in Section 7.2, the PDT introduced in Refs. [13,16,51,58,59,63,64,181] for the 1D Hubbard model has been extended to simpler integrable models such as the present 1D Lieb-Liniger Bose gas [61] and the spin-1/2 XXX chain [62]. The PDT is associated with the pseudofermion representation of such models. One of the goals of this review is to clarify the relation between the PDT and the MQIM methods [52,53,57]. For simplicity, below in Section 7.4 and Appendix A the present 1D Lieb-Liniger Bose gas is used to address that problem. The basic relation is qualitatively similar for the more complex models also reviewed in this paper. The relation of the 1D Lieb-Liniger Bose gas PDT to the MQIM of Ref. [57] allows the expression of the general PDT $\iota= \pm$ pseudofermion Fermi points fluctuations functionals, Eq. (37), in terms of the MQIM shift function $F_{B}\left(k \mid k^{\prime}\right)$ defined in Eqs. (7) and (8) of Ref. [57]. In that reference it is called $F_{B}(v \mid \mu)$ whereas here its variables $v$ and $\mu$ are replaced by our notation for the momentum rapidities, $k$ and $k^{\prime}$, respectively. Furthermore, the corresponding limiting values $\pm q$ are replaced by our notation $\pm Q$ for them, Eq. (11). From the use of the relation $\Phi\left(\iota q_{F}, q_{j^{\prime}}\right)=\frac{\xi^{1}}{2}-F_{B}\left(\iota k_{0}\left(q_{F}\right), k_{0}\left(q_{j^{\prime}}\right)\right)$, Eq. (A.9) of Appendix A, one readily finds that,

$$
2 \Delta^{\iota}\left(\left\{q_{j^{\prime}}\right\}\right)=\left(\xi^{1}\left(\delta J_{b}^{F}+\frac{\delta N_{b}^{N F}}{2}\right)+\iota \frac{\delta N_{b}^{F}}{2 \xi^{1}}-\sum_{j^{\prime}=1}^{\infty} \delta N^{N F}\left(q_{j^{\prime}}\right) F_{B}\left(\iota Q \mid k_{0}\left(q_{j^{\prime}}\right)\right)\right)^{2} \text { for } \iota= \pm,
$$

where $\delta N_{b}^{N F}=\sum_{j^{\prime}=1}^{\infty} \delta N^{N F}\left(q_{j^{\prime}}\right) \leq N_{b}^{N F}=\sum_{j^{\prime}=1}^{\infty}\left|\delta N^{N F}\left(q_{j^{\prime}}\right)\right|$.

By considering low-energy excited states for which $\delta N^{N F}\left(q_{j^{\prime}}\right)=0$ for all $q_{j^{\prime}}$ values away from the $\iota= \pm$ pseudofermion Fermi points, the functionals, Eqs. (37) and (39), acquire the simplified form, Eq. (38). Hence both the PDT and the MQIM naturally contain the present model low-energy conformal field theory. In the case of the PDT, the link of the functionals to the conformal dimensions in the correlation functions obtained from the BA [174-176] can be understood as described in the following.

The property that the excitation energy spectrum, Eq. (35), has no pseudofermion energy interactions simplifies the expression of the dynamical correlation functions in terms of pseudofermion spectral functions. The lack of energy interactions achieved under the pseudoparticle-momentum pseudofermion-canonical-momentum transformation $q_{j} \rightarrow$ $q_{j}+2 \pi \Phi\left(q_{j}\right) / L$, Eq. (33), has though a price: The usual integer or half-integer dynamical correlation functions exponents of non-interacting and Fermi-liquid like quantum systems are replaced by the interaction and momentum dependent exponents whose expressions involve the $\iota= \pm$ functional dimensions, Eqs. (37) and (39).

The pseudofermion representation involves a mere unitary transformation under which the integer or half-integer BA quantum numbers $I_{j}$ in $q_{j}=\frac{2 \pi}{L} I_{j}$, Eqs. (3) and (4), are shifted to $I_{j} \rightarrow \bar{I}_{j}$ where $\bar{I}_{j}=I_{j}+\Phi\left(q_{j}\right)$. The pseudofermion phase shift $\Phi\left(q_{j}\right)$ in units of $2 \pi$ appearing here, Eq. (33), is in general both interaction and momentum dependent. The dynamical correlation functions usual integer or half-integer dimensions are mapped under the transformation associated with such a shift, $I_{j} \rightarrow I_{j}+\Phi\left(q_{j}\right)$, onto the exotic interaction and momentum dependent functionals, Eqs. (37) and (39).

This is why the exponents that control the dynamical correlation functions line shape in the vicinity of well-defined types of $(k, \omega)$-plane singular spectral features are interaction and momentum dependent functionals. In the low-energy limit, the general $\iota= \pm$ functional dimensions $2 \Delta^{\prime}\left(\left\{q_{j^{\prime}}\right\}\right)$ in Eqs. (37) and (39) where $j^{\prime}=1, \ldots, N_{b}^{N F}$ lose their momentum dependence. Indeed, they refer to excited states for which $N_{b}^{N F}=0$. However they remain being interaction dependent since they become the $\iota= \pm$ fields conformal dimensions $2 \Delta_{0}^{\iota}$, Eq. (38). 
In the case of the more complex 1D Hubbard model, the PDT contains as well the conformal field theory as a limiting behavior. (For further technical information on the link of the generalized dimensions, Eqs. (37) and (39), to the conformal dimensions in the correlation functions also obtained from the BA and how the PDT leads in the low-energy limit to exactly the same correlation functions as conformal-field theory, see Ref. [181].)

The generalized functional dimensions in Eqs. (37) and (39) correspond to an important step beyond conformal-field theory. They apply actually both at low and high energy. Hence one can learn from the pseudofermion representation new insights beyond the model low-energy physics. Indeed, the additional deviations $\sum_{j^{\prime}=1}^{\infty} \delta N^{N F}\left(q_{j^{\prime}}\right) \Phi\left(\iota q_{F}, q_{j^{\prime}}\right)$ in Eq. (37) actually control the high-energy regime of dynamical correlation functions. The corresponding generalized dimensions, Eqs. (37) and (39), are confirmed in the following and in Sections 7.2 and 7.4 to play an important role in the dynamical correlation functions spectral weight distributions. For instance, the interaction and momentum dependent exponents that control the dynamical correlation functions line shape in the vicinity of well-defined types of $(k, \omega)$-plane singular spectral features are within the PDT a superposition of the such generalized dimensions. This refers to the vicinity of these functions $(k, \omega)$-plane lower or upper thresholds. In the case of one-particle spectral functions, this applies as well near a particular type of singular features called within the PDT branch lines.

Within the PDT the dynamical correlation functions are written in terms of pseudofermion spectral functions. Such functions spectral weights can be expressed as Slater determinants written in terms of anticommutators of pseudofermion operators. (For simplicity, in the case of the present model we do not introduce here the corresponding pseudofermion operator algebra.) The Slater determinants are written in terms of anticommutators of pseudofermion operators. Their expressions involve the overall phase-shift functional,

$$
2 \pi \Phi^{T}\left(q_{j}\right)=2 \pi \Phi^{0}+2 \pi \Phi\left(q_{j}\right)=2 \pi \Phi^{0}+\sum_{j^{\prime}=1}^{\infty} \delta N\left(q_{j^{\prime}}\right) 2 \pi \Phi\left(q_{j}, q_{j^{\prime}}\right) .
$$

The corresponding dynamical correlation functions one- and two-boson spectral weights are written in terms of such anticommutators. Their dependence on the phase-shift functional $2 \pi \Phi^{T}\left(q_{j}\right)$ is the mechanism through which the shake up effects occurring in the pseudofermion canonical momentum band under the transitions to the excited states lead to the Anderson's orthogonality catastrophes [182]. Such a shake up refers to the change from the ground-state momentum values $q_{j}^{0}$ to the excited states canonical momentum values $q_{j}^{0}+2 \pi \Phi^{T}\left(q_{j}\right) / L=q_{j}+2 \pi \Phi\left(q_{j}\right) / L$. (Here $q_{j}=q_{j}^{0}+2 \pi \Phi^{0} / L$ are the pseudoparticle momentum values of the excited state.)

The different nature of the dynamical correlation functions of the pseudofermion quantum liquid relative to those of a Fermi liquid originates from these Anderson's orthogonality catastrophes. Those are associated with finite contributions to the one- and two-boson spectral weight distributions from a large number of low-energy and small-momentum particlehole processes in the pseudofermion band. The form of the functional dimensions, Eq. (37) and (39), results from these contributions [61].

In the following we provide the momentum dependent exponents obtained from the PDT. Those control the line shape of the one-boson addition and removal spectral functions and two-boson charge dynamical structure factor near their thresholds. These dynamical correlation functions for the boson problem were studied first by the MQIM [57]. Recently the use of the PDT reached exactly the same spectra and momentum dependent exponents for such functions [61]. (In Ref. [57] analytical expressions for these exponents were derived yet their momentum dependence has not been plotted.)

The one-boson removal and addition spectral functions are given by [57,61],

$$
S^{B}(k, \omega) \equiv B(k, \omega)=\sum_{f}\left|\left\langle f, N_{b}-1\left|\hat{\Psi}_{k}\right| G S, N_{b}\right\rangle\right|^{2} \delta\left(\omega-\omega^{B}(k)\right),
$$

and

$$
S^{A}(k, \omega) \equiv A(k, \omega)=\sum_{f}\left|\left\langle f, N_{b}+1\left|\hat{\Psi}_{k}^{\dagger}\right| G S, N_{b}\right\rangle\right|^{2} \delta\left(\omega-\omega^{A}(k)\right),
$$

respectively. Here $\omega^{B}(k)=E_{G S}^{N_{b}}-E_{f}^{N_{b}-1}, \omega^{A}(k)=E_{f}^{N_{b}+1}-E_{G S}^{N_{b}}, E_{n}$ is the excited states energy, $E_{0}$ that of the ground state, and $\hat{\Psi}_{k}^{\dagger}$ and $\hat{\Psi}_{k}$ are boson creation and annihilation operators. Furthermore, the charge dynamical structure factor reads [57,61,183],

$$
S^{D}(k, \omega) \equiv S(k, \omega)=\sum_{f}\left|\left\langle f\left|\hat{\rho}_{k}\right| G S\right\rangle\right|^{2} \delta\left(\omega-\omega^{D}(k)\right),
$$

where $\omega^{D}(k)=E_{f}-E_{G S}$ and $\hat{\rho}_{k}$ is the Fourier transform of the local density operator $\hat{\rho}_{x}$. As mentioned above, the charge dynamical structure factor, Eq. (43), can be probed in ultra-cold atom systems through low-momentum Bragg excitations [33,34],

The $(k, \omega)$-plane lower $\left(c_{\tau}=-1\right)$ or upper $\left(c_{\tau}=1\right)$ thresholds of the energy spectra of the above dynamical spectral functions have for the momentum range $k \in\left[0,2 \pi n_{b}\right]$ the general form,

$$
\omega^{\tau}=c_{\tau} \varepsilon\left(q_{F}-k\right) \text { where } k=q_{F}-q \in\left[0,2 \pi n_{b}\right] .
$$



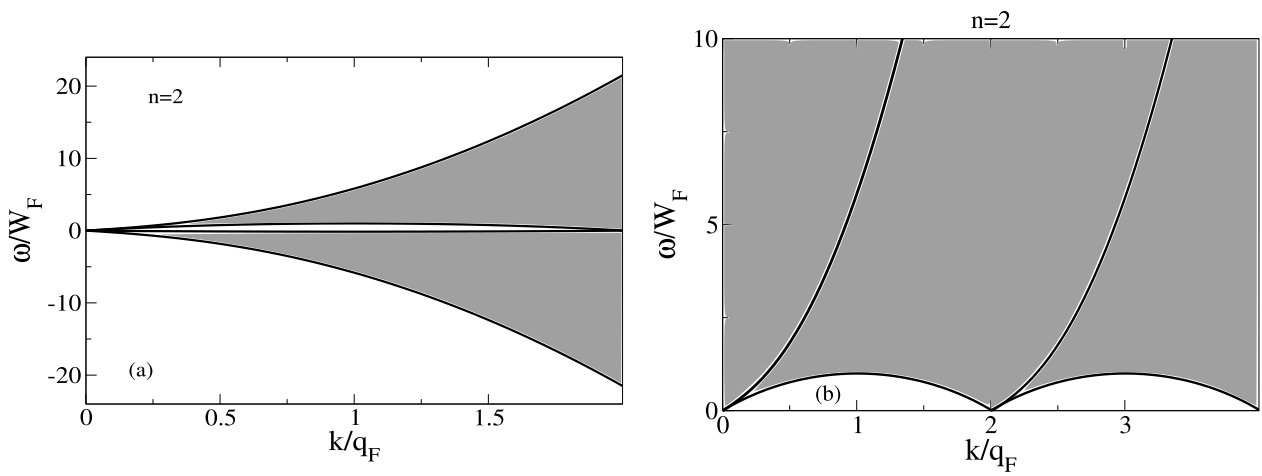

Fig. 3. The spectra, Eq. (44), of the excitations associated with the dominant contributions to the one-boson removal $(\omega<0)$ and addition $(\omega>0)$ spectral function (left panel) and charge dynamical structure factor (right panel) for $c=1$ and density $n_{b}=2$. In the case of the latter factor, the spectrum edge for $k / q_{F} \in[2,4]$ and the weight above it associated with the next-order excitations has also been included. The momentum scale $q_{F}$ and energy scale $W_{F}$ are the pseudoparticle Fermi momentum, Eq. (7), and energy bandwidth of the ground-state occupied Fermi sea, Eq. (14), respectively. (As in Ref. [61], $n_{b}$ is denoted by $n$ in the figures.)

Source: From Ref. [61].

Here $\varepsilon(q)$ is the energy dispersion, Eq. (11). The index $\tau$ reads $\tau=B$ for the one-boson removal spectral function, $\tau=A$ for the one-boson addition spectral function, and $\tau=D$ for the two-boson dynamical structure factor. The coefficient $c_{\tau}$ is given by $c_{\tau}=1$ for $\tau=B$ and $c_{\tau}=-1$ for $\tau=A$, D. The spectra, Eq. (44), are shown in Fig. 3 for $c=1$ and density $n_{b}=2$.

For small energy deviations $\left(\omega-\omega^{\tau}(k)\right)>0$ in the vicinity of the $(k, \omega)$-plane lower $\left(c_{\tau}=-1\right)$ or upper $\left(c_{\tau}=1\right)$ thresholds, the PDT leads to the following exact line shape [61],

$$
S^{\tau}(k, \omega)=C^{\tau}\left(\omega-\omega^{\tau}(k)\right)^{\xi_{\tau}(k)} \text { for } k \in\left[0,2 \pi n_{b}\right] \text { where } \tau=B, A, D .
$$

Here $C^{\tau}$ is a coefficient whose value remains unchanged in the range of small energy deviations $\left(\omega-\omega^{\tau}(k)\right)>0$ for which this expression is valid. The momentum dependent exponents are given by,

$$
\xi_{\tau}(k)=-1+\sum_{\iota= \pm} 2 \Delta_{\tau}^{\iota}=-1+\sum_{\iota= \pm}\left(\frac{\xi^{1}}{2}+\iota \frac{b_{\tau}}{\xi^{1}}-\Phi\left(\iota q_{F}, q_{F}-k\right)\right)^{2} .
$$

The $\iota= \pm$ functional dimensions in this expression, $2 \Delta_{\tau}^{\iota}=\left(\delta \bar{q}_{F}^{\iota} /(2 \pi / L)\right)^{2}$, are those given in Eq. (37) for the excited states specific to each of the $\tau=B, A, D$ dynamical correlation functions, $b_{\tau}=0$ for $\tau=B, b_{\tau}=1$ for $\tau=A$, and $b_{\tau}=1 / 2$ for $\tau=D$. The one-boson removal upper threshold and addition lower threshold are in Fig. 3 the two boundary lines above and below the $\omega=0$ axis, respectively.

The spectral feature in Eqs. (44)-(46) is called a branch line [61]. It is generated by elementary processes where only one pseudofermion is created (and annihilated) outside the Fermi points. When in the $(k, \omega)$ plane there is no spectral weight above (lower threshold) or below (upper threshold) that line, the corresponding dynamical correlation function analytical expression is exact. If above or below it there is a very small amount of spectral weight, it is an approximation.

The latter approximation is valid for one-particle spectral functions whose spectral-weight distribution is not plateaulike. In contrast, this is the general case for two-particle dynamical correlation functions. However, in the case of the 1D LiebLiniger Bose gas charge dynamical structure factor, the spectral weight between the spectrum edge line for $k / q_{F} \in[2,4]$ in Fig. 3 and the rising branch line in that figure starting at $k / q_{F}=2$ vanishes in the $n_{b} / c \rightarrow \infty$ limit [61]. Hence we consider here the charge dynamical structure factor for very large $n_{b} / c$ values in the vicinity of that branch line, which has the form,

$$
\omega^{D}=\varepsilon\left(k-q_{F}\right) \text { where } k=q+q_{F} \in\left[2 \pi n_{b}, \infty\right] \text { for } q \in\left[\pi n_{b}, \infty\right] .
$$

For small $\left(\omega-\omega^{D}(k)\right)>0$ values near it, an approximation for the dynamical structure factor is,

$$
S^{D}(k, \omega) \approx C^{D}\left(\omega-\omega^{D}(k)\right)^{\xi_{D}(k)} \text { for } k \in\left[2 \pi n_{b}, \infty\right] .
$$

The momentum dependent exponent is here of the form,

$$
\xi_{D}(k)=-1+\sum_{\iota= \pm}\left(\frac{\xi^{1}}{2}-\frac{\iota}{2 \xi^{1}}+\Phi\left(\iota q_{F}, k-q_{F}\right)\right)^{2} .
$$

The two $\tau=B, A$ one-boson spectral function exponents $\xi_{\tau}(k)$, Eq. (46), and the dynamical structure factor exponent $\xi_{D}(k)$, Eq. (49), are plotted in Fig. 4 as a function of the momentum $k$ in units of $q_{F}=\pi n_{b}$ for interaction $c=1$ and several densities $n_{b}$ values. For $k / q_{F}>2$ the latter exponent is an approximation. For the ranges $k / q_{F} \in\left[0, k^{*} / q_{F}\right]$ (where $k^{*}$ is such that $\xi_{B}\left(k^{*}\right)=0$ ) in Fig. 4 for which the exponent $\xi_{B}(k)$ is negative, there are upper threshold singularity cusps in the one-boson removal spectral function $S^{B}(k, \omega)$, Eq. (41). 

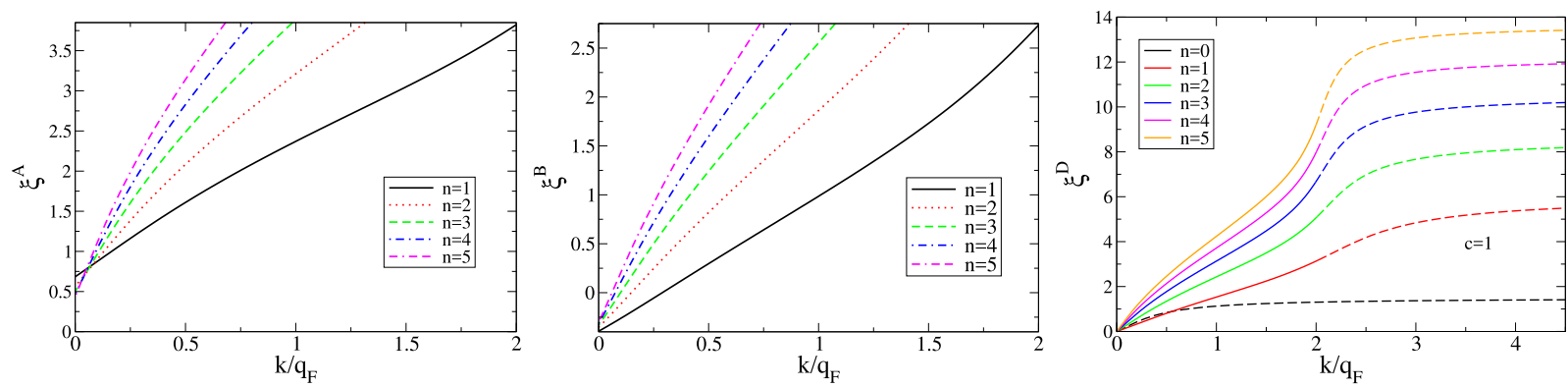

Fig. 4. The exponents, Eq. (46), $\xi_{A}(k)$ of the one-boson addition spectral function, $\xi_{B}(k)$ of the one-boson removal spectral function, and $\xi_{D}(k)$ of the charge dynamical structure factor for $c=1$ plotted as a function of the momentum in units of $q_{F}$ for the density $n_{b}$ values given in the figure panels. (The momentum scale $q_{F}$ and energy scale $W_{F}$ are those of Fig. 3.) The latter exponent is exact for $k / q_{F}<2$ (solid lines) and an approximation for $k / q_{F}>2$ (dashed lines). For $k / q_{F}>2$ it is a better approximation for the values $c=1$ and $n_{b}=5$ for which $n_{b} / c$ is largest. Only $\xi_{B}(k)$ has negative values for a limited momentum range associated with upper threshold singularity cusps in the one-boson removal spectral function. (As in Ref. [61], $n_{b}$ is denoted by $n$ in the figures.)

Source: From Ref. [61].

\section{The spin-1/2 isotropic Heisenberg chain}

The spin- $1 / 2 X X X$ chain is again a quantum problem of interest. It is a paradigmatic example of an integrable strongly correlated system that is experimentally relevant for the description of magnetic properties of spin-chain materials $[133,134,136,184,185]$. At zero magnetic field the model contains antiferromagnetic correlations for exchange integral $J>0$. Those have been observed in dynamical quantities measured in inelastic neutron scattering experiments on spin-chain compounds [136]. It can also be prepared in a 1D ultra-cold atomic trap [32].

Here we consider the spin-1/2 XXX chain in the TL. In that limit the complex rapidities in the BA equations simplify in terms of the ideal strings of Ref. [72]. (The deviations from such ideal strings [131] do not affect in the TL the properties of the model revisited in this section.)

The Hamiltonian of the general spin-1/2 Heisenberg chain with exchange integral $J>0$ and anisotropy parameter $\Delta \geq 0$ in a magnetic field $H$ reads,

$$
\begin{aligned}
& \hat{H}_{\Delta}=J \sum_{j=1}^{L}\left(\sum_{\tau=x, y} \hat{S}_{j}^{\tau} \hat{S}_{j+1}^{\tau}+\Delta \hat{S}_{j}^{z} \hat{S}_{j+1}^{z}\right)+2 \mu_{B} H \sum_{j=1}^{L} \hat{S}_{j}^{z} \\
& \text { where } \hat{S}^{\tau}=\sum_{j=1}^{L} \hat{S}_{j}^{\tau} \text { and } \hat{S}^{ \pm}=\sum_{j=1}^{L} \hat{S}_{j}^{ \pm} \text {with } \tau=x, y, z .
\end{aligned}
$$

Here $\hat{\vec{S}}_{j}$ and $\hat{S}^{\tau}$ are the spin-1/2 operators at site $j=1, \ldots, L$ with components $\hat{S}_{j}^{x, y, z}$ and related total spin operators, respectively, $\hat{S}_{j}^{ \pm}=\hat{S}_{j}^{x} \pm i \hat{S}_{j}^{y}$, and $\mu_{B}$ is the Bohr magneton.

At the isotropic point, $\Delta=1$, the model contains antiferromagnetic correlations that have been observed in dynamical quantities measured in experiments on spin-chain compounds [133,136,184,185],

$$
\hat{H}=J \sum_{j=1}^{L} \hat{\vec{S}}_{j} \cdot \hat{\vec{S}}_{j+1}+2 \mu_{B} H \sum_{j=1}^{L} \hat{S}_{j}^{z} .
$$

Another observable of interest for our study of this model is the $z$ component of the spin current operator,

$$
\hat{J}^{z}=-i J \sum_{j=1}^{L}\left(\hat{S}_{j}^{+} \hat{S}_{j+1}^{-}-\hat{S}_{j+1}^{+} \hat{S}_{j}^{-}\right) .
$$

\subsection{The model physics: fractional excitations, spin ordering, and magnetism}

To address the phenomena brought about by quantum magnetic correlations, it is instructive to consider systems where the charge degrees of freedom are frozen and only spin excitations remain. Such systems are usually described by spinonly models. They are realized, for example, in Mott insulators where magnetic interactions between the local moments of localized electrons are mediated by virtual exchange processes between neighboring electrons. One can describe the magnetic correlations through models of localized quantum spins embedded on lattices. At zero magnetic field local 
moment antiferromagnetic phases frequently occur in the arena of strongly correlated electron systems. The phenomenology displayed by the antiferromagnetic Hamiltonian depends sensitively on the geometry of the underlying lattice.

On the one hand, on a bipartite lattice of dimension $d>1$, such as the square lattice, i.e. one in which the neighbors of one sub-lattice A belong to the other sub-lattice B, the zero-field ground state of the spin-1/2 isotropic Heisenberg antiferromagnet is close to a staggered spin configuration. It is known as a Néel state whose neighboring spins are antiparallel. The linear spin-wave theory for antiferromagnets [186] relies onto an expansion that is valid provided that $\frac{1}{N} \sum_{k}\left\langle n_{k}\right\rangle \ll 1$. Here $n_{k}$ is the occupation number of the spin-wave state of momentum $k$. For the square lattice one finds $\frac{1}{N} \sum_{k}\left\langle n_{k}\right\rangle \approx 0.197$, which is indeed a rather small number. The elementary excitations above the zero-field antiferromagnetic ground state are then spin waves of two flavors, due to the two sub-lattices. The elementary particle of a spin wave is called a magnon. It carries a spin equal to one.

On the other hand, for the spin chain, the lattice is trivially bipartite. However, in the 1D case one rather finds that $\frac{1}{N} \sum_{k}\left\langle n_{k}\right\rangle \rightarrow \infty$ at zero magnetic field [187]. This is due to the long-wavelength modes. Indeed, it is well known that the zero-field ground state of the spin chain fails to develop long-range antiferromagnetic order. As a result, in 1D there is no zero-field linear spin-wave theory small expansion parameter. This is why the perturbative expansion around an ordered zero-field ground state is incorrect for the spin-1/2 XXX chain.

The zero magnetic field spin order and associated magnetism of that spin chain is thus of a different type. Fortunately, in spite of its non-perturbative nature and lack of zero-field ground-state long-range order, one can extract important information on that quantum problem physics from its exact BA solution. Rather than spin waves and magnons, the elementary excitations of the zero-field spin-1/2 XXX chain, which correspond to well-defined BA quantum numbers distributions, are often associated with phenomenological nontrivially interacting spin-1/2 particles called spinons. Their energy dispersion relation is extracted from the BA $[66,67,75,78,79]$. (The phenomenological/empirical nature of the spinons stems from their precise operational relation to the model physical spins not being defined.)

Consider some $S U(2)$-symmetric Hamiltonian, in more than one spatial dimension, made out of spin- $1 / 2$ particles, which is in a ground state that spontaneously breaks the $S U(2)$ symmetry. If one flips a single spin, a magnon is created, not a spinon. Spinons that emerge in spin chains are much weirder. Since any local spin operator changes an integer amount of spin, one cannot create a single spinon with a local operator. Hence spinons are examples of fractionalized particles: They can only arise as part of a physical disturbance.

The spin-1/2 XXX chain whose Hamiltonian is given in Eq. (51) is a paradigmatic example of both a spin chain and an integrable strongly correlated quantum many-body system. In this paper we review the related pseudoparticle and pseudofermion representations of its exact BA solution. Such a solution refers explicitly to the lowest-weight states (LWSs) or highest-weight states (HWSs) of the $S U(2)$ algebra for which $S=-S^{z}$ and $S=S^{z}$, respectively. Here (and in the following) the spin and spin projection of the spin chain, Eq. (51), energy eigenstates have been denoted by $S$ and $S^{z}=-\left(L_{\uparrow}-L_{\downarrow}\right) / 2$, respectively. $L_{\sigma}$ such that $\sum_{\sigma=\uparrow, \downarrow} L_{\sigma}=L$ denotes the number of physical spins of projection $\sigma=\uparrow, \downarrow$. (The sign choice in the expression $S^{z}=-\left(L_{\uparrow}-L_{\downarrow}\right) / 2$ is the same as in Ref. [72], for which $L_{\uparrow} \geq L_{\downarrow}$ for a LWS.) The pseudoparticle and pseudofermion representations also applies to the extended Hilbert space spanned by both the LWSs and corresponding multiplet spin $S U(2)$ towers of states.

The BA gives direct access to the energy levels of an integrable system. This allows the computation of many equilibrium quantities. The ground state energy of the spin-1/2 XXX chain was derived analytically within the TL in the early stages of its studies [173]. However, it was not until the 1960s and early 1970s that its excitation spectrum was computed [68] and its thermodynamic properties derived [72,188-192]. Equilibrium quantities are, nonetheless, not sufficient to completely characterize the physics of correlated models.

The computation of dynamical quantities requires knowledge of matrix elements of spin operators between energy eigenstates. This goes beyond the information that can be extracted directly from the BA. At zero field some of such states are described by groups of real and complex BA rapidities. The spin dynamical structure factors are objects that motivated partially by experimental work have been extensively studied in the case of zero magnetic field [114,193-198]. In Section 3.7 we thus revisit the spin dynamical structure factors of the spin-1/2 XXX chain for the less studied case of a finite magnetic field.

For a finite magnetic field $H>0$ the Hamiltonian term $2 \mu_{B} H \sum_{j=1}^{L} \hat{S}_{j}^{z}$ in Eq. (51) does not commute with the global spin $S U(2)$ symmetry off-diagonal generators. It thus lowers the model global symmetry to $U(1)$. However, for all spin density values $m=-2 S^{z} / L \in[-1,1]$ and corresponding fields $h \in\left[-H_{c}, H_{c}\right]$ the $S$-fixed subspaces dimensions, and thus the number of energy and momentum eigenstates that span them, exactly equals the number of fixed- $S$ representations of the $S U$ (2) symmetry group. Here $\pm H_{c}= \pm J / \mu_{B}$ are the critical fields for fully polarized ferromagnetism. Hence the $H=0$ model non-Abelian global $S U(2)$ symmetry controls and determines the states spectrum structure for all $m$ and $H$ values.

\subsection{A functional representation of the spin-1/2 XXX chain Bethe-ansatz solution}

The model Hamiltonian, Eq. (51), is solvable by the BA. The corresponding general BA equation is of the form [40,72],

$$
2 \arctan \left(\Lambda_{j}\right)=q_{j}+\frac{1}{L} \sum_{\alpha \neq j} 2 \arctan \left(\frac{\Lambda_{j}-\Lambda_{\alpha}}{2}\right) \text { where } \bmod 2 \pi .
$$


Here the $\alpha=1, \ldots,(L-2 S) / 2$ summation is over the subset of occupied $q_{\alpha}$ quantum numbers out of the full set,

$$
q_{j}=\frac{2 \pi}{L} I_{j} \text { for } j=1, \ldots, I_{S} \text { where } I_{S}=(L+2 S) / 2 .
$$

The different occupancy configurations of the related quantum numbers $I_{j}$ (defined modulo $L$ ) such that $j=1, \ldots, I_{S}$ generate different energy and momentum eigenstates. The latter are successive integers or half-odd integers according to the boundary conditions,

$$
\begin{aligned}
I_{j} & =0, \pm 1, \ldots, \pm \frac{I_{S}-1}{2} \text { for } I_{S} \text { odd } \\
& = \pm 1 / 2, \pm 3 / 2, \ldots, \pm \frac{I_{S}-1}{2} \text { for } I_{S} \text { even } .
\end{aligned}
$$

The BA equation, Eq. (53), explicitly refers to the LWSs. However, relying on the model spin $S U(2)$ symmetry one can extend its exact solution to the non-LWSs. That global $S U(2)$ spin symmetry imposes that the energy and momentum eigenstates refer to state representations of the group $S U(2)$. Consistently, the LWSs and the non-LWSs generated from them used in our analysis are energy and momentum eigenstates. They are as well eigenstates of $(\hat{\vec{S}})^{2}$ and $\hat{S}^{z}$ with eigenvalues $S(S+1)$ and $S^{z}$, respectively. We thus denote all $2^{L}$ energy and momentum eigenstates by $\left|l_{\mathrm{r}}, S, S^{z}\right\rangle$. Here $l_{\mathrm{r}}$ stands for all quantum numbers other than $S$ and $S^{z}$ needed to specify a state, $\left|l_{\mathrm{r}}, S, S^{z}\right\rangle$. The non-LWSs are generated from the corresponding $n_{s}=S+S^{z}=0$ LWS $\left|l_{\mathrm{r}}, S,-S\right\rangle$ as,

$$
\left|l_{\mathrm{r}}, S, S^{z}\right\rangle=\frac{1}{\sqrt{\mathcal{C}}}\left(\hat{S}^{+}\right)^{n_{S}}\left|l_{\mathrm{r}}, S,-S\right\rangle \text { where } \mathcal{C}=\left(n_{s} !\right) \prod_{j=1}^{n_{S}}(2 S+1-j) \text { for } n_{s}=1, \ldots, 2 S .
$$

The BA wave functions of the LWSs $\left|l_{\mathrm{r}}, S,-S\right\rangle$ formally vanish when two rapidities $\Lambda_{j}$ and $\Lambda_{j^{\prime}}$ become equal (Fermi-like statistics.) This property suggests that simply choosing $\alpha=1, \ldots,(L-2 S) / 2$ distinct quantum numbers $q_{\alpha}$ among the set of $j=1, \ldots, I_{S}$ allowed quantum numbers $q_{j}$, which gives a dimension $\left(\begin{array}{c}(L+2 S) / 2 \\ (L-2 S) / 2\end{array}\right)$, would allow the reconstruction of all $2^{L}$ states that span the model Hilbert space.

However such an expectation is misleading. Indeed due to the model non-Abelian global spin $S U(2)$ symmetry and in contrast to the simpler $U(1)$ symmetry 1D Lieb-Liniger Bose gas, only some of the solutions to the general BA equation, Eq. (53), are obtained in terms of real rapidities $\Lambda_{j}$. The model non-Abelian symmetry gives rise to new internal degrees of freedom absent from that Bose gas. Those bring about new BA roots that involve groups of complex rapidities [40,72]. In the context of our study, the term thermodynamic Bethe ansatz (TBA) refers to the form obtained in Ref. [72] for the BA equations in the TL. Within the TBA, the needed set of real and complex rapidities have the general form,

$$
\Lambda_{j}^{n, l}=\Lambda_{j}^{n}+i(n+1-2 l) \text { such that } \Lambda_{j}^{n, l}=\left(\Lambda_{j}^{n, n+1-l}\right)^{*} \text { where } l=1, \ldots, n,
$$

$j=1, \ldots, L_{n}$ with $n=1, \ldots, \infty$, and the number $L_{n} \geq N_{n}$ is defined below. As confirmed in the following, the extra solutions and corresponding states associated with the quantum numbers in the complex rapidities, Eq. (57), ensure that in each $S$-fixed subspace the number of such states equals that of state representations of the model global spin $S U(2)$ symmetry.

For $n=1$ the rapidity, Eq. (57), is real and otherwise its imaginary part is finite. The rapidities are roots of Eq. (53). In Eq. (57) they are partitioned in a configuration of strings, where a $n$-string is a group of $n$ rapidities with the same real part $\Lambda_{j}^{n}$. The number $n$ is often called the string length and the real part of the set of $n$ rapidities, $\Lambda_{j}^{n}$, is called the string center [1].

After some algebra, the use of rapidities of the form given in Eq. (57) in the general BA equation, Eq. (53), leads to a number $n=1, \ldots,(L-2 S) / 2$ of TBA equations. In general we consider that $n=1, \ldots, \infty$ in the TL, which is correct provided that $\left(1-L_{s}\right)$ is finite. Within the momentum-distribution functional notation used in this review, the TBA equations read $[87,88]$,

$$
q_{j}=k_{j}^{n}-\frac{1}{L} \sum_{\left(n^{\prime}, j^{\prime}\right) \neq(n, j)} N_{n^{\prime}}\left(q_{j^{\prime}}\right) \Theta_{n n^{\prime}}\left(\Lambda_{j}^{n}-\Lambda_{j^{\prime}}^{n^{\prime}}\right) .
$$

Here $\Theta_{n n^{\prime}}(x)$ is an odd function of $x$ given in Eq. (B.1) of Appendix B where $n, n^{\prime}=1, \ldots, \infty$. In that equation and throughout this review $\delta_{n, n^{\prime}}$ is the usual Kronecker symbol.

The solutions of the TBA equations, Eq. (58), define the rapidities real part, $\Lambda_{j}^{n}$. In these $n=1, \ldots, \infty$ equations,

$$
q_{j}=\frac{2 \pi}{L} I_{j}^{n} \in\left[q_{n}^{-}, q_{n}^{+}\right] \text {where } j=1, \ldots, L_{n} \text { and } q_{n}^{ \pm}= \pm \frac{\pi}{L}\left(L_{n}-1\right),
$$

are the momentum values of a $n$-band associated with the set of $N_{n} n$-strings with the same $n$ value. The quantum numbers $I_{j}^{n}$ are successive integers or half-odd integers according to the boundary conditions,

$$
\begin{aligned}
I_{j}^{n} & =0, \pm 1, \ldots, \pm \frac{L_{n}-1}{2} \text { for } L_{n} \text { odd } \\
& = \pm 1 / 2, \pm 3 / 2, \ldots, \pm \frac{L_{n}-1}{2} \text { for } L_{n} \text { even } .
\end{aligned}
$$


The distribution function $N_{n}\left(q_{j}\right)$ in Eq. (58) is such that $N_{n}\left(q_{j}\right)=1$ and $N_{n}\left(q_{j}\right)=0$ for "occupied" and "unoccupied" $q_{j}$ values, respectively. Indeed, the $q_{j}$ values, Eq. (59), have the separation, $q_{j+1}-q_{j}=2 \pi / L$, and only occupancies zero and one. For each fixed $n$ there is a number $L_{n}$ of $q_{j}$ values given by $[72,87,88]$,

$$
L_{n}=N_{n}+N_{n}^{h} \text { where } N_{n}^{h}=2 S+N_{n}^{h, 0} \text { and } N_{n}^{h, 0}=\sum_{n^{\prime}=n+1}^{\infty} 2\left(n^{\prime}-n\right) N_{n^{\prime}} .
$$

For consistency with the notation used for the 1D Lieb-Liniger Bose gas, here we have called $N_{n}$ where $n=1, \ldots, \infty$ the quantum numbers named $M_{n}$ in the TBA studies of Ref. [72]. Otherwise we tend to use the notations and formalism of that reference. $N_{n}$ and $N_{n}^{h}$ are in Eq. (61) the numbers of $q_{j}$ values that are occupied and unoccupied, respectively, and $N_{n}^{h, 0}$ is the latter number in the case of a $S=0$ energy and momentum eigenstate. Often an index $\alpha=1, \ldots, N_{n}$ is used to label the subset of occupied quantum numbers $I_{\alpha}^{n}$ of an energy and momentum eigenstate [72,87,88]. Moreover, in Eq. (58),

$$
k_{j}^{n} \equiv k^{n}\left(q_{j}\right)=2 \arctan \left(\frac{\Lambda_{j}^{n}}{n}\right) .
$$

(The relation of the $n=1$ rapidity momentum $k_{j}^{1}=2 \arctan \left(\Lambda_{j}^{1}\right)$, Eq. (62) for $n=1$, to the rapidity momentum $k_{j}$ of Ref. [72], such that $\Lambda_{j}^{1}=\cot \left(k_{j} / 2\right)$, is $k_{j}^{1}=\pi-k_{j}$.)

The momentum eigenvalues $P$ and the energy eigenvalues $E$ are functionals of the $n=1, \ldots, \infty$ distribution functions $N_{n}\left(q_{j}\right)$ given by,

$$
P=\pi+\sum_{n=1}^{\infty} \sum_{j=1}^{L_{n}} N_{n}\left(q_{j}\right) q_{j} \text { and } E=-\sum_{n=1}^{\infty} \sum_{j=1}^{L_{n}} N_{n}\left(q_{j}\right) \frac{J}{n}\left(1+\cos k_{j}^{n}\right)-2 \mu_{B} H S^{z},
$$

respectively.

The form of the momentum eigenvalues confirms that the quantum number variables $q_{j}$ defined in Eq. (54) such that $q_{j+1}-q_{j}=2 \pi / L$ play the role of $n$-band momentum values. There is one such momentum band for each set of $N_{n} n$-strings with the same length $n=1, \ldots, \infty$. For a given energy eigenstate, each such a $n$ band has a well-defined set of $N_{n}$ occupied and $N_{n}^{h}$ unoccupied momentum values $q_{j}$. The full set of $L_{n}=N_{n}+N_{n}^{h}$ momentum values $q_{j}$ is distributed within an interval $q_{j} \in\left[q_{n}^{-}, q_{n}^{+}\right]$. Its limiting values $q_{n}^{ \pm}$are given in Eq. (59) and Eq. (B.3) of Appendix B.

In the ensuing section it is confirmed that the set of quantum numbers associated with the TBA equations, Eq. (58), allows the reconstruction of the $2^{L}$ energy eigenstates that span the spin-1/2 XXX chain full Hilbert space. Out of such $\sum_{2 S=0 \text { (integers) }}^{L} \mathcal{N}(S)=2^{L}$ states, there is for a given $S$ a number $\mathcal{N}(S)=(2 S+1) \mathcal{N}_{\text {singlet }}(S)$ of states. Those correspond to $(2 S+1)$ multiplet configurations and a number $\mathcal{N}_{\text {singlet }}(S)$ singlet configurations given in Eq. (C.1) of Appendix C.

\subsection{The n-pseudoparticles representation of the spin-1/2XXX chain Bethe-ansatz roots and its relation to the paired physical spins} $1 / 2$

The pseudoparticle representation introduced in the following is in the case of the XXX chain associated with its $L$ physical spins $1 / 2$ rather than with spinons. The advantage is that such a representation is valid for the model full Hilbert and parameter spaces. The energy eigenstates are a superposition of lattice occupancy configurations in which the $L$ physical spins $1 / 2$ singly occupy $L$ lattice sites. $L$ is even and odd when the states spin $S$ is an integer and half-odd integer number, respectively. For all states that span a fixed-S subspace, the corresponding lattice occupancy configurations have then a number $2 S$ of sites occupied by a set of $M=2 S$ physical spins $1 / 2$ that participate in the multiplet configuration. The complementary set of even number $L-2 S$ of sites are singly occupied by $L-2 S$ physical spins $1 / 2$ whose configuration forms a tensor product of singlet states.

Since all the $\mathcal{N}(S)$ energy eigenstates with the same $S$ value have the same $\hat{\vec{S}}^{2}$ eigenvalue, the energy and momentum eigenstates are superpositions of such configuration terms. Each term is characterized by a different partition of $L$ physical spins $1 / 2$ into $2 S$ such physical spins that participate in a $2 S+1$ multiplet, and a product of singlets involving the remaining even number $L-2 S$ of physical spins $1 / 2$. The latter are associated with a corresponding number $(L-2 S) / 2$ of singlet pairs.

The unpaired spins $1 / 2$ and paired spins $1 / 2$ are the members of such two sets of $M \equiv 2 S$ and $2 \Pi \equiv L-2 S$ physical spins $1 / 2$, respectively. For a LWS, all physical unpaired spins $1 / 2$ have up spin projection. The model TBA solution quantum numbers are directly related to such $M \equiv 2 S$ up physical spins $1 / 2$ and different types of singlet configurations involving the remaining $2 \Pi=L-2 S$ paired physical spins $1 / 2$ and their $\Pi=(L-2 S) / 2$ singlet pairs.

A $n$-string was defined above as a group of $l=1, \ldots, n$ rapidities with the same real part $\Lambda_{j}^{n}$, Eq. (57). As confirmed in the following, the set of $n$-strings of an energy eigenstate is directly related to the $\Pi \equiv(L-2 S) / 2$ singlet pairs involving the $2 \Pi=L-2 S$ physical spins $1 / 2$ that participate in singlet configurations. Specifically, each $n$-string refers to a $n$-pairs configuration within which for $n>1$ a number $n$ of singlet pairs are bound. Such a binding is associated with the corresponding imaginary parts, $i(n+1-2 l)$, of the $l=1, \ldots, n$ rapidities $\Lambda_{j}^{n, l}=\Lambda_{j}^{n}+i(n+1-2 l)$, Eq. (57), with the same real part $\Lambda_{j}^{n}$. The $n>1$ singlet pairs that are bound within a $n$-pairs configuration associated with a string of length $n>1$ are called here bound singlet pairs. For $n=1$ the rapidity $\Lambda_{j}^{1,1}$ imaginary part vanishes because a $n=1 n$-string 
refers to a single singlet pair. The $N_{1}$ unbound singlet pairs of an energy eigenstate are those that correspond its $N_{1} n=1$ pair configurations.

The rapidity $\Lambda_{j}^{n, l}$ indexes $n$ and $l$ thus label the $n$-pairs configuration and a specific singlet pair, respectively. Moreover, the usual string length $n=1, \ldots, \infty$ in Eq. (57) corresponds to the number of singlet pairs in each of the $N_{n} n$-pairs configurations of a given state. The $l=1, \ldots, n$ singlet pairs bound within a $n$-pairs configuration involve a number $2 n$ of physical spins $1 / 2$. Those singly occupy $2 n$ lattice sites.

Consistently with such a relation between the TBA $n$-strings and the $\Pi=(L-2 S) / 2$ singlet pairs, the following exact TBA sum rule holds for all energy eigenstates,

$$
\Pi=\sum_{n=1}^{\infty} n N_{n}=\frac{1}{2}(L-2 S),
$$

and thus $\pi=\Pi / L=\sum_{n=1}^{\infty} n n_{n}=\frac{1}{2}\left(1-m_{s}\right)$. Here $N_{n}$ is the number of $n$-pairs configurations that equals that of $q_{j}$ values that are occupied, $\pi$ is the density of singlet pairs, $m_{s}=2 S / L=M / L \geq m$ that of unpaired spins $1 / 2$, and $n_{n}=N_{n} / L$. The $\Pi=(L-2 S) / 2$ singlet pairs under consideration involve the $2 \Pi=L-2 S$ physical spins $1 / 2$ that do not participate in multiplet configurations.

The physical spins $1 / 2$ configurations that generate an energy eigenstate are a superposition of local lattice occupancy configurations. On the one hand, since each $n$-pairs configuration occupies a number $2 n$ of lattice sites, the set of $n$-pairs configurations of an energy eigenstate occupy a number $2 \Pi=\sum_{n=1}^{\infty} 2 n N_{n}$ of lattice sites. On the other hand, each of the $M=2 S$ unpaired physical spins $1 / 2$ singly occupies a lattice site. Therefore, for an energy eigenstate of spin $S$, the following number of sites sum rule is fulfilled,

$$
L=M+2 \Pi=2 S+\sum_{n=1}^{\infty} 2 n N_{n} .
$$

There is a strong requirement for each $n$-string referring to a $n$-pairs configuration that involves a number $2 n$ of physical spins $1 / 2$ and corresponding $l=1, \ldots, n$ singlet pairs: That in the dimension of any $S$-fixed subspace $\mathcal{N}(S)=$ $(2 S+1) \mathcal{N}_{\text {singlet }}(S)$, the number of independent singlet configurations $\mathcal{N}_{\text {singlet }}(S)$ be exactly the same when obtained from the counting of two apparently different types of configurations. The first refers to the counting of the $S U(2)$ group state representations associated with the physical spins $1 / 2$ independent configurations with the same spin $S$, Eq. (C.1) of Appendix C. The second corresponds to the counting of independent $n=1, \ldots, \infty$ bands $\left\{q_{j}\right\}$ occupancy configurations of the sets of $N_{n} n$-strings obeying the sum rule $\sum_{n=1}^{\infty} n N_{n}=(L-2 S) / 2$, Eq. (64). (The factor $(2 S+1)$ in the dimension $\mathcal{N}(S)=(2 S+1) \mathcal{N}_{\text {singlet }}(S)$ refers to the number of multiplet configurations of the $M=2 S$ unpaired physical spins $1 / 2$. Those are not part of $n$-pairs configurations singlet pairs.)

As shown in Appendix A of Ref. [72] for LWSs, the value of the number $N_{n}^{h}=L_{n}-N_{n}$ of $n$-band holes that naturally emerges from the TBA, Eq. (61), ensures that for each $S$-fixed subspace the singlet dimension $\mathcal{N}_{\text {singlet }}(S)$ can indeed alternatively be written as given in Eqs. (C.1) and (C.2) of Appendix C, respectively. This also holds for the multiplet towers of non-LWS generated from $S>0$ LWSs. Indeed, all $2 S+1$ states of such a tower have exactly the same singlet configurations as the corresponding $S>0$ LWS.

The summation $\sum_{\left\{N_{n}\right\}}$ in Eq. (C.2) of Appendix C runs over all sets of $n$-strings numbers $\left\{N_{n}\right\}$ corresponding to the same fixed spin $S=L / 2-\sum_{n=1}^{\infty} n N_{n}$, as imposed by the exact sum rule, Eq. (64). On the one hand, this confirms the connection between $n$-strings and the paired physical spins $1 / 2$. On the other hand, it shows that the additional states described by groups of complex rapidities, absent in the case of the 1D Lieb-Liniger Bose gas, ensure that the fixed-S subspaces dimension, $\mathcal{N}(S)=(2 S+1) \mathcal{N}_{\text {singlet }}(S)$ with $\mathcal{N}_{\text {singlet }}(S)$ given in Eq. (C.2) of Appendix C, exactly equals the corresponding number of spin- $S$ $S U(2)$ symmetry representations, Eq. (C.1) of that Appendix.

From analysis of the TBA equations given in the previous section, one finds that there is a one-to-one correspondence between the $N_{n} n$-pairs configurations with the same number $n$ of singlet pairs of an energy eigenstate and the $N_{n}$ occupied momentum values $q_{j}$ of the corresponding $n$-band distribution $N_{n}\left(q_{j}\right)$, respectively. This is consistent with the center of mass of the set of the $2 n$-sites occupied by each $n$-pairs configuration moving with momentum $q_{j}$ and all its $2 n$ sites singly occupied by paired physical spins moving coherently along with it. This occurs through processes within which the $2 n$ paired spins on such $2 n$ occupied sites interchange position with the $M=2 S$ unpaired physical spins $1 / 2$ that singly occupy sites.

We associate one n-pseudoparticle and one $n$-band hole with each of the $N_{n}$ occupied and $N_{n}^{h}$ unoccupied momentum values $q_{j}$, respectively, of an energy eigenstate $n$-band. Such pseudoparticles are well defined within the TL to which the TBA applies. Indeed, the TL ensures that the problems concerning the $n$-pseudoparticle internal degrees of freedom and translational degrees of freedom, respectively, separate.

On the one hand, the internal degrees of freedom of a $n$-pseudoparticle refer to a $n$-pairs configuration. Hence there is one $n$-pseudoparticle for each $n$-pairs configuration and corresponding BA roots. Those involve a group of $l=1, \ldots, n$ rapidities with the same real part, Eq. (57). If $n>1$ the $n$-pseudoparticle has $n=2, \ldots, \infty$ singlet pairs bound within it. If $n=1$, its internal degrees of freedom correspond to a single unbound singlet pair. That a $n$-string of length $n>1$ describes the binding of $n=2, \ldots, \infty$ singlet pairs bound within a $n$-pseudoparticle clarifies its connection to the BA roots.

On the other hand, the momentum $q_{j}$, Eq. (59), of a $n$-pseudoparticle refers to its translational degrees of freedom. Those are associated with its center of mass motion. The set of $N=\sum_{n=1}^{\infty} N_{n} n$-pseudoparticles, each carrying a momentum $q_{j}$, of a given energy eigenstate determine that state momentum eigenvalue, as given in Eq. (63). 
The magnons associated with the spin-wave representation of the spin-1/2 XXX model on for example a square lattice carry spin one and are associated with an antiferromagnetic long-range order. In turn, the $l=1, \ldots, n$ singlet pairs bound within the $N_{n} n$-pseudoparticles that populate each $n=1, \ldots, \infty n$-band of an energy eigenstate of the present spin chain have spin zero. The $n$-pseudoparticles are indeed spin neutral particles. The energy eigenstates spin $S$ and spin projection $S^{z}$ are thus determined by the numbers $M_{ \pm 1 / 2}$ of unpaired spins $1 / 2$ with spin projection $\pm 1 / 2$. Specifically, $S=\left(M_{+1 / 2}+M_{-1 / 2}\right) / 2$ and $S^{z}=-\left(M_{+1 / 2}-M_{-1 / 2}\right) / 2$, respectively. The total number $L_{ \pm 1 / 2}$ of physical spins with projection $\pm 1 / 2$ such that $L=L_{+1 / 2}+L_{-1 / 2}$ then reads $L_{ \pm 1 / 2}=\Pi+M_{ \pm 1 / 2}$.

There is a number of pseudoparticles sum rule. It is related to that of singlet pairs, Eq. (64). The latter sum rule implies that $N_{1}=L\left(1-L_{s}\right) / 2-\sum_{n=2}^{\infty} n N_{n}$. From the use of this relation in the number of pseudoparticles expression, $N \equiv \sum_{n=1}^{\infty} N_{n}$, one confirms that the sum rule $N=\sum_{n=1}^{\infty} N_{n}=\frac{1}{2}\left(L-N_{1}^{h}\right)$ is obeyed. Here $N_{1}^{h}$ is the number of $n=1$ band holes, Eq. (61) for $n=1$.

The set of $\Pi=\sum_{n=1}^{\infty} n N_{n}=(L-2 S) / 2$ singlet pairs of an energy eigenstate are all bound within the set of $N=\sum_{n=1}^{\infty} N_{n}$ composite $n$-pseudoparticles that populate it. The question is thus which is the relation of the model physical spins $1 / 2$ to the $N_{n}^{h}=2 S+N_{n}^{h, 0}$ holes in each $n$-band for which $N_{n}>0$ ? The $N_{1}^{h} n=1$ band holes of the $S=0$ ground-state excited states are usually associated with spinons $[65,199]$. Hence this question also refers to the relation of spinons to the present representation in terms $n$-pseudoparticles and unpaired spins $1 / 2$. This is the issue clarified in the ensuing section.

\subsection{Relation to the physical spins $1 / 2$ of the holes in the TBA quantum numbers distributions}

The spin-1/2 XXX chain in a uniform vector potential $\Phi / L$ whose Hamiltonian is given in Eq. (A2) of Ref. [87] remains solvable by the BA. Its LWSs momentum eigenvalues, $P=P(\Phi / L)$, have the general form,

$$
P(\Phi / L)=P(0)+\frac{L-\sum_{n} 2 n N_{n}}{L} \Phi=P(0)+L_{S} \Phi=P(0)+2 S \frac{\Phi}{L} .
$$

The $\Phi=0$ momentum eigenvalue $P(0)$ is given in Eq. (63).

On the one hand, the current operator expectation values of the $\Phi \rightarrow 0$ LWSs can be derived from the $\Phi / L$ dependence of the energy eigenvalues $E(\Phi / L)$ as $\left\langle\hat{J}^{z}\right\rangle=d E(\Phi / L) /\left.d(\Phi / L)\right|_{\Phi=0}$. On the other hand, $d P(\Phi / L) /\left.d(\Phi / L)\right|_{\Phi=0}$ gives the number of spin carriers that couple to the vector potential. The natural candidates are the model $L$ physical spins $1 / 2$. However, the form of the exact momentum eigenvalues, Eq. (66), reveals though that only the $2 S$ unpaired spins $1 / 2$ contributing to the multiplet configurations couple to the vector potential $\Phi / L$. Since the $2 \Pi=L-2 S$ physical spins $1 / 2$ left over are those within the $\Pi=L / 2-S$ singlet pairs, this result is physically appealing.

The LWSs spin currents result from the above mentioned processes under which the $2 n$-site configurations of the $n$-pseudoparticles interchange position under their motion along the lattice with the set of single-site $2 S$ unpaired spins. The BA separates each $n=1, \ldots, \infty$ branch of $n$-pseudoparticles in a different momentum $n$ band. The inequality $N_{n}^{h} \geq 2 S$ follows from the BA choosing the number $N_{n}^{h}$ of $n$ band holes so that the fixed-S subspaces dimension obeys the sum rule, Eq. (C.2) of Appendix C. The additional number $\sum_{n^{\prime}=n+1}^{\infty} 2\left(n^{\prime}-n\right) N_{n^{\prime}}$ of holes in each $n$ band relative to the number $2 S$ that equals that of unpaired spins $1 / 2$ has an interpretation in terms of lattice sites occupancies. Indeed, locally it corresponds to $\sum_{n^{\prime}=n+1}^{\infty} 2\left(n^{\prime}-n\right) N_{n^{\prime}}$ sites out of the $\sum_{n^{\prime}=n+1}^{\infty} 2 n^{\prime} N_{n^{\prime}}$ sites occupied by bound singlet pairs within the state under consideration. And this refers only to $n^{\prime}$-pairs configurations with $n^{\prime}>n$ such pairs. Only such sites in addition to the $2 S$ sites occupied by unpaired spins are used by the $n$ pseudoparticles as unoccupied sites. They interchange position under their motion along the lattice with the spins $1 / 2$ on such $\sum_{n^{\prime}=n+1}^{\infty} 2\left(n^{\prime}-n\right) N_{n^{\prime}}$ lattice sites. They are though occupied by paired spins $1 / 2$ with an equal number $\sum_{n^{\prime}=n+1}^{\infty}\left(n^{\prime}-n\right) N_{n^{\prime}}$ of opposite spin projections rather than by unpaired spins $1 / 2$. Therefore, such processes do not contribute to the spin current.

Indeed, on average only $2 S$ holes out of the $N_{n}^{h}=2 S+\sum_{n^{\prime}=n+1}^{\infty} 2\left(n^{\prime}-n\right) N_{n^{\prime}}$ holes of each $n$ band for which $N_{n}>0$ contribute to the spin currents. The translational degrees of freedom of the $2 S$ unpaired spins $1 / 2$ are described by such $n$-band holes. On average the virtual elementary currents carried by the two sets of $\sum_{n^{\prime}=n+1}^{\infty}\left(n^{\prime}-n\right) N_{n^{\prime}}$ remaining holes exactly cancel each other. This is consistent with the overall spin current of $S=0$ states for which $N_{n}^{h}=N_{n}^{h, 0}=$ $\sum_{n^{\prime}=n+1}^{\infty} 2\left(n^{\prime}-n\right) N_{n^{\prime}}$ exactly vanishing.

The validity of this picture is confirmed by the form of the spin currents of the non-LWSs. Consider a general LWS $\left|l_{\mathrm{r}}, S,-S\right\rangle$ on the right-hand side of Eq. (56) carrying a current $\left\langle l_{\mathrm{r}}, S,-S \hat{J}^{z} \mid l_{\mathrm{r}}, S,-S\right\rangle$. Here $\hat{J}^{z}$ is the $z$ component of the spin current operator, Eq. (52). For simplicity, we denote that spin current by $\left\langle\hat{V}_{L W S}^{z}\left(l_{\mathrm{r}}, S\right)\right\rangle$. In Appendix $\mathrm{E}$ it is shown that its general TBA expression $[87,88]$ can be written in terms of $n$-bands holes elementary currents $j_{n}^{h}\left(q_{j}\right)$ as follows,

$$
\left\langle\hat{J}_{L W S}^{z}\left(l_{\mathrm{r}}, S\right)\right\rangle=\sum_{n=1}^{\infty} \sum_{j=1}^{L_{n}} N_{n}^{h}\left(q_{j}\right) j_{n}^{h}\left(q_{j}\right) .
$$

Here $N_{n}^{h}\left(q_{j}\right)=1-N_{n}\left(q_{j}\right)$ and $l_{\mathrm{r}}$ labels the $\sum_{l_{\mathrm{r}}}=\mathcal{N}_{\text {singlet }}(S)=\sum_{\left\{N_{n}\right\}} \prod_{n=1}^{\infty}\left(\begin{array}{l}L_{n} \\ N_{n}\end{array}\right)$ independent singlet configurations of the $L-2 S$ paired spins $1 / 2$. Such configurations correspond to a well-defined set of numbers $\left\{N_{n}\right\}$ of $n$-pairs configurations. Those are associated with the energy and momentum eigenstates that span each fixed- $S$ subspace. The $n$-bands holes elementary 
currents $j_{n}^{h}\left(q_{j}\right)$ in Eq. (67) are determined by the LWS rapidity functions $k^{n}\left(q_{j}\right)$ obtainable from solution of the TBA equations, Eq. (B.1) of Appendix B. They read,

$$
j_{n}^{h}\left(q_{j}\right)=\frac{2 J \sin k^{n}\left(q_{j}\right)}{2 \pi \sigma^{n}\left(k^{n}\left(q_{j}\right)\right)} \text { for } q_{j} \in\left[q_{n}^{-}, q_{n}^{+}\right] .
$$

The distribution $2 \pi \sigma^{n}\left(k_{j}\right)$ appearing here is within the TL given by $\left.2 \pi \sigma^{n}\left(k_{j}\right) \equiv 2 \pi \sigma^{n}(k)\right|_{k=k_{j}}$ where $2 \pi \sigma^{n}(k)=d q^{n}(k) / d k$. $q^{n}(k)$ stands in that derivative for the inverse function of the $n$-band rapidity momentum function $k^{n}(q)$.

We consider now that $L$ is even and thus the states spin $S$ is an integer number. However, within the TL the same results are reached for $L$ odd. All $2 S$ unpaired spins $1 / 2$ of that LWS have up-spin projection. The following exact relation between the spin currents of the non-LWSs belonging to the same spin $S U(2)$ tower and the spin current of the corresponding LWS in terms of the numbers $M_{ \pm 1 / 2}$ of unpaired spins $1 / 2$ with spin projection $\pm 1 / 2$ holds [87,88],

$$
\left\langle\hat{J}^{z}\left(l_{\mathrm{r}}, M_{+1 / 2}, M_{-1 / 2}\right)\right\rangle=\frac{\left(M_{+1 / 2}-M_{-1 / 2}\right)}{2 S}\left\langle\hat{J}_{L W S}^{z}\left(l_{\mathrm{r}}, S\right)\right\rangle .
$$

For each spin flip generated by application of the off-diagonal spin generator $\hat{S}^{+}$in Eq. (56) (and $\hat{S}^{-}$) onto a state with finite numbers $M_{+1 / 2}$ and $M_{-1 / 2}$, the spin current, Eq. (69), exactly changes by a LWS current quantum $2 j_{-1 / 2}$ (and $2 j_{+1 / 2}$ ). The elementary currents $j_{ \pm 1 / 2}$ in such quanta are given by,

$$
j_{ \pm 1 / 2}= \pm \frac{\left\langle\hat{J}_{L W S}^{z}\left(l_{\mathrm{r}}, S\right)\right\rangle}{2 S}= \pm \frac{\sum_{n=1}^{\infty} \sum_{j=1}^{L_{n}} N_{n}^{h}\left(q_{j}\right) j_{n}^{h}\left(q_{j}\right)}{2 S} .
$$

Hence each unpaired spin $1 / 2$ with spin projection $\pm 1 / 2$ carries such an elementary current $j_{ \pm 1 / 2}$. For LWSs one has that $M_{+1 / 2}=2 S$ and $M_{-1 / 2}=0$ whereas $M_{+1 / 2}+M_{-1 / 2}=2 S$ for their non-LWSs. The general expression for the number of $n$-band holes thus reads $N_{n}^{h}=M_{+1 / 2}+M_{-1 / 2}+\sum_{n^{\prime}=n+1}^{2} 2\left(n^{\prime}-n\right) N_{n^{\prime}}$. For each of the $2 S+1$ states in the same $S U(2)$ tower, an average number $M_{+1 / 2}$ and $M_{-1 / 2}$ of holes in the $n$ bands for which $N_{n}>0$ describe the translational degrees of freedom of the $M_{+1 / 2}$ and $M_{-1 / 2}$ unpaired spins $1 / 2$ of spin projection $+1 / 2$ and $-1 / 2$, respectively.

The exact spin current expression, Eq. (69), is proportional to $M_{+1 / 2}-M_{-1 / 2}$. The currents of two sets of $M_{+1 / 2}$ and $M_{-1 / 2}$ unpaired spins of opposite spin projection then partially or totally $\left(M_{+1 / 2}=M_{-1 / 2}\right)$ cancel each other. Therefore, only an average number $\left|M_{+1 / 2}-M_{-1 / 2}\right|$ of holes in the $n$ bands for which $N_{n}>0$ contribute to the spin current. The virtual elementary currents of a corresponding average number $2 S_{\eta}-\left|M_{+1 / 2}-M_{-1 / 2}\right|+\sum_{n^{\prime}=n+1}^{\infty} 2\left(n^{\prime}-n\right) N_{n^{\prime}}$ of holes in these bands exactly cancel each other. For $S_{\eta}^{z}=0$ non-LWSs for which $M_{+1 / 2}=M_{-1 / 2}$ this is a total canceling. Such states have zero spin current.

The spin stiffness $D(T)$ in the real part of the spin conductivity Drude peak, $2 \pi D(T) \delta(\omega)$, is for temperatures $T \geq 0$ an important physical quantity related to spin ballistic transport. Indeed, a finite spin stiffness implies the occurrence of such a type of transport. At finite temperature $T>0$ the spin stiffness can within the TL and for a fixed- $S^{z}$ canonical ensemble be expressed only in terms of the spin currents, Eq. (69), of all energy and momentum with that $S^{z}$ value $[87,88]$. It then follows that in the TL and within the canonical ensemble the spin stiffness of the spin-1/2XXX chain vanishes as $m^{z}=\left|2 S^{z}\right| \rightarrow 0$ for $T>0$. At zero temperature the spin stiffness expression has additional contributions from off-diagonal matrix elements of the spin current operator. Some of those do not vanish in the $m^{z} \rightarrow 0$ limit. The zero-temperature spin stiffness is thus finite in the TL for $m^{z} \rightarrow 0$, as found below in Section 3.6. (It is given in Eq. (91).)

There is a direct relation between quantum spin transport and both local and quasi-local conservation laws [201-203]. For the present model, the spin stiffness can be accessed by suitable use of the TBA [200]. It can also be accessed employing a hydrodynamic description. Within it, the spin stiffness is calculated from the stationary currents generated in an inhomogeneous quench from bipartitioned initial states [204]. That in the $m^{z} \rightarrow 0$ limit the spin stiffness of the spin-1/2 $X X X$ chain is within the TL finite at $T=0$ and vanishes for $T>0[87,88]$ is illustrated in Fig. 5 . The stiffness curves plotted in that figure were calculated by use of the TBA in Ref. [200] for the anisotropic spin-1/2 1D Heisenberg chain, Eq. (50). (The $X X X$ chain refers to the $\Delta=1$ isotropic point in the figure.)

There is a connection between ballistic and diffusive transport in the $m^{z} \rightarrow 0$ limit at nonzero temperatures when the ballistic contribution of the spin-1/2 XXX chain vanishes [205].

\subsection{The spinon representation as a limiting case of the n-bands hole representation}

Consider LWSs for which the numbers $M=2 S$ of physical unpaired spins $1 / 2$ and $N_{n}^{h}=2 S+N_{n}^{h, 0}$ of holes in $n$ bands for which $n>1$ are within the TL finite. The densities of unpaired spins $1 / 2$ and $n=1$ band holes of such states thus behave in the TL as $m_{s}=2 S / L \rightarrow 0$ and $n_{1}^{h}=N_{1}^{h} / L \rightarrow 0$, respectively. One has then that $q_{n}^{ \pm} \rightarrow 0$ for such $n>1$ bands whose momentum bandwidth vanishes. For LWSs for which $m_{s} \ll 1$ and $n_{1}^{h} \ll 1$ one finds that the $n$-bands hole elementary currents in Eq. (68) are given by $j_{n}^{h}\left(q_{j}\right)=J \frac{(n-1)}{3 n}\left(2 \pi n_{1}^{h}\right)^{2} \sin \left(\frac{q_{j}}{m_{n}}\right)$ for $n>1$. Hence $j_{n}^{h}\left(q_{j}\right) \rightarrow 0$ for the above class of LWSs.

An important quantum problem refers to the spin-1/2 XXX chain in the reduced subspace spanned by the above states for which $m_{s} \rightarrow 0$ and $n_{n}^{h} \rightarrow 0$ for $n>1$ as $L \rightarrow \infty$. For it the expression of the LWS spin current, Eq. (67), and general 


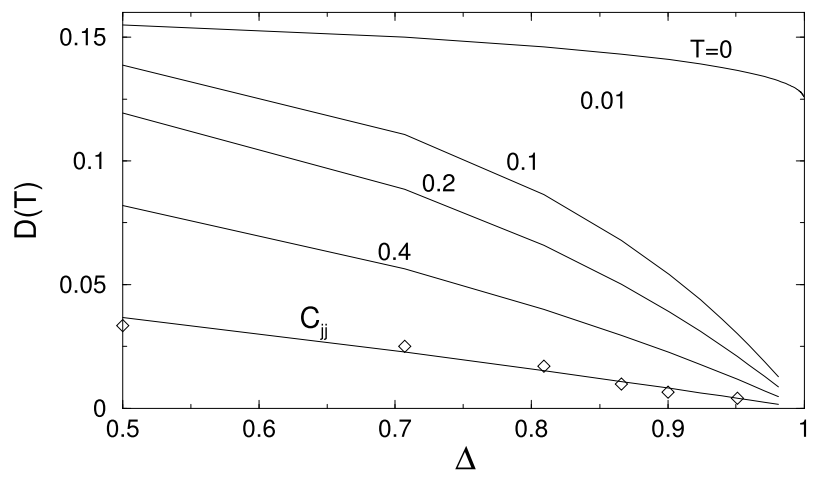

Fig. 5. The spin stiffness of the spin-1/2 1D Heisenberg chain, Eq. (50), as a function of the anisotropy parameter $\Delta$ at various temperatures. $\left(C_{j j}\right.$ is a high-temperature proportionality constant [200].)

Source: From Ref. [200].

current, Eq. (69), simplifies to,

$$
\begin{aligned}
\left\langle\hat{U}_{L W S}^{z}\left(l_{\mathrm{r}}, S\right)\right\rangle & =\sum_{j=1}^{L_{1}} N_{1}^{h}\left(q_{j}\right) j_{1}^{h}\left(q_{j}\right) \text { and } \\
\left\langle\hat{J}^{z}\left(l_{\mathrm{r}}, M_{+1 / 2}, M_{-1 / 2}\right)\right\rangle & =\frac{\left(M_{+1 / 2}-M_{-1 / 2}\right)}{2 S} \sum_{j=1}^{L_{1}} N_{1}^{h}\left(q_{j}\right) j_{1}^{h}\left(q_{j}\right)=\sum_{\iota= \pm} M_{\iota 1 / 2} \times j_{\iota 1 / 2},
\end{aligned}
$$

respectively. Here,

$$
j_{ \pm 1 / 2}= \pm \frac{\sum_{j=1}^{L_{1}} N_{1}^{h}\left(q_{j}\right) j_{1}^{h}\left(q_{j}\right)}{2 S} \text { and } j_{1}^{h}\left(q_{j}\right)=\frac{2 J \sin k^{1}\left(q_{j}\right)}{2 \pi \sigma^{1}\left(k^{1}\left(q_{j}\right)\right)} \text { for } q_{j} \in\left[q_{1}^{-}, q_{1}^{+}\right],
$$

are elementary currents, Eq. (70), carried by a unpaired physical spin $1 / 2$ of projection $\pm 1 / 2$ and the $n=1$ band elementary current spectrum, Eq. (68) for $n=1$, respectively.

The spinon representation [65,199] applies to such a quantum problem. For it the expressions in Eqs. (71) and (72) are valid. Such expressions also refer to energy and momentum eigenstates of arbitrary spin $S$ described only by groups of real rapidities. The spinon representation also applies to them.

Within such a representation, the spinons are the $N_{1}^{h}=2 S+N_{1}^{h, 0}$ holes in the $n=1$ band $[65,199]$. It is assumed that each spinon carries a spin $1 / 2$. For LWSs described by groups of real and complex rapidities for which $N_{1}^{h, 0}=\sum_{n=2}^{\infty} 2(n-1) N_{n}$ is finite, $N_{1}^{h, 0}$ gives the number of such spinons in singlet configurations. The elementary currents of such spinons vanish. This result is confirmed by the use of the BA solution for small $N_{1}^{h, 0}$ values. In contrast, the currents of an average number $2 S$ of remaining spinons contribute to the spin current. The latter $2 S$ spinons are intended to be a representation of the model $2 S$ unpaired physical spins $1 / 2$. In the case of LWSs described only by groups of real rapidities, one has that $N_{1}^{h, 0}=0$ and $N_{1}^{h}=2 S$. For such states the $N_{1}^{h}=2 S$ spinons are intended to describe the $2 S$ unpaired physical spins $1 / 2$.

Each of the $N_{1}^{h}$ spinons of a LWS has been inherently constructed to carry an elementary current $j_{1}^{h}\left(q_{j}^{h}\right)$. This is consistent with the LWS overall spin current reading $\sum_{j=1}^{L_{1}} N_{1}^{h}\left(q_{j}\right) j_{1}^{h}\left(q_{j}\right)$, Eq. (71). The spinon representation has an empirical character. This follows for it not providing operational relation of the spinons to the model physical spins $1 / 2$. A relevant question is thus whether a spinon carrying an elementary current $j_{1}^{h}\left(q_{j}^{h}\right)$, Eq. (72), has indeed internal degrees of freedom associated with a spin $1 / 2$ operator algebra?

This issue remains hidden in the case of LWSs. For them the spin current is the sum $\left\langle\hat{J}_{L W S}^{z}\left(l_{\mathrm{r}}, S\right)\right\rangle=\sum_{j=1}^{L_{1}} N_{1}^{h}\left(q_{j}\right) j_{1}^{h}\left(q_{j}\right)$, Eq. (71), of the elementary currents $j_{1}^{h}\left(q_{j}^{h}\right)$ associated with the $N_{1}^{h}$ spinons. It can be clarified though if one considers the tower of non-LWSs corresponding to each LWS. It is useful to compare the spin currents obtained for the spin LWS and HWS BA solutions. This reveals that if a spinon with a given momentum $q_{j}^{h}$ carried one spin $1 / 2$, its elementary current would read $\pm j_{1}^{h}\left(q_{j}^{h}\right)$ in the case of spin projection $\pm 1 / 2$. This would imply that one spin flip resulting from the application of the spin $S U(2)$ off-diagonal generator $\hat{S}^{ \pm}$onto the non-LWS under consideration would lead to state spin current changes $\mp 2 j_{1}^{h}\left(q_{j}^{h}\right)$.

This is though in contrast to the exact expression of the general spin current $\left\langle\hat{J}^{z}\left(l_{\mathrm{r}}, M_{+1 / 2}, M_{-1 / 2}\right)\right\rangle$ given in Eq. (71). That expression reveals that the spin current changes under consideration rather read $2 j_{\mp 1 / 2}=\mp\left[\sum_{j=1}^{L_{1}} N_{1}^{h}\left(q_{j}\right) j_{1}^{h}\left(q_{j}\right)\right] /(2 S)$ for the non-LWSs belonging to the spin $S U(2)$ towers of the LWSs considered here. This shows one cannot associate the internal degrees of freedom of one spin $1 / 2$ with a BA discrete quantum number $n=1$ band hole momentum $q_{j}^{h}$. Hence such internal degrees of freedom can neither be associated with the corresponding spinon. 
Each of the $N_{1}^{h}$ spinons with a given momentum $q_{j}^{h}$ carrying one spin $1 / 2$ is appealing in the case of LWSs. In that case the spinon elementary currents provide a faithful representation of the state overall spin current. However, the $n=1$ band holes associated with the spinons are mere neutral particles. An average number $2 S$ of them merely describes the translational degrees of freedom of the $2 S$ physical unpaired spins $1 / 2$. However, they lack their spin $1 / 2$ internal degrees of freedom. Such degrees of freedom are rather located on the $2 S$ sites occupied by the physical unpaired spins $1 / 2$ within the local configurations whose superposition generates a state.

The spinon representation does not apply for general states with an arbitrary finite density $m_{s}=2 S / L=M / L$ of unpaired spins $1 / 2$ described by groups of real and complex rapidities. This is because the expressions in Eqs. (71) and (72) do not account for all contributions to the spin current in Eqs. (67)-(70) of such general states. Indeed, such currents are not determined by the "spinons" occupancy configurations alone. For such states, a number $2 S$ of holes in each $n$ band for which $N_{n}>0$ contributes to the spin current. And this applies to all $n>1$ bands with finite $n$-pseudoparticle occupancy. Hence the spinon representation is in this general case replaced by the extended $n$-bands hole representation considered in Section 3.4. For it, the expressions in Eqs. (71) and (72) that only involve $n=1$ band holes are replaced by those given in Eqs. (67)-(70).

An extreme example refers to the LWSs whose $\Pi=L / 2-S$ singlet pairs are all bound within a single gigantic $n=\Pi=L / 2-S$ pairs-configuration. The spin current $\left\langle\hat{J}_{L W S}^{z}\left(l_{\mathrm{r}}, S\right)\right\rangle=-2 J \sin q_{j}$ of these LWSs stems entirely from the $2 S$ holes in the $n=\Pi=L / 2-S$ band. Specifically, it results from their motion upon exchanging position with the single gigantic pseudoparticle of momentum $q_{j}$. This spin current has thus no contribution whatsoever from the holes in the $n=1$ band. Indeed they do not exist because $N_{1}=0$ for such states.

\subsection{The $n=1$ band pseudoparticles quantum liquid}

For a LWS ground state the $n$-band limiting values $q_{n}^{ \pm}$in Eq. (59) and Eq. (B.3) of Appendix B are given by,

$$
q_{1}^{ \pm}= \pm \frac{\pi}{L}\left(L_{1}-1\right)= \pm k_{F \uparrow} \text { and } q_{n}^{ \pm}= \pm \frac{\pi}{L}\left(L_{n}-1\right)= \pm\left(k_{F \uparrow}-k_{F \downarrow}\right) \text { for } n>1 .
$$

Here,

$$
k_{F \uparrow}=\frac{\pi}{2 L}(L+2 S-2) \approx \frac{\pi}{2}(1+m) \text { and } k_{F \downarrow}=\frac{\pi}{2 L}(L-2 S-2) \approx \frac{\pi}{2}(1-m) .
$$

Furthermore, for such a ground state the $n$-pseudoparticle momentum distribution functions in Eq. (58), read,

$$
N_{1}^{0}\left(q_{j}\right)=\theta\left(q_{F}-\left|q_{j}\right|\right) \text { and } N_{n}^{0}\left(q_{j}\right)=0 \text { for } n>1 .
$$

The $n=1$ band Fermi momentum appearing here is given by,

$$
q_{F}=k_{F \downarrow}=\frac{\pi}{2 L}(L-2 S-2) \approx \frac{\pi}{2}(1-m) .
$$

Ground states are not populated by $n$-pseudoparticles for which $n>1$. In the case of the $n=1$ band, within the TL one can classify the deviations $\delta N_{1}\left(q_{j}\right)$ in Eq. (77) as $\delta N_{1}^{F}\left(q_{j}\right)$ and $\delta N_{1}^{N F}\left(q_{j}\right)$, respectively. On the one hand, for the deviations $\delta N_{1}^{F}\left(q_{j}\right)$ the band momentum $q_{j}$ is such that $\lim _{L \rightarrow \infty}\left(\left|q_{j}\right|-k_{F \downarrow}\right)=0$. On the other hand, in the case of $\delta N_{1}^{N F}\left(q_{j}\right)$ the momentum difference $\lim _{L \rightarrow \infty}\left(\left|q_{j}\right|-k_{F \downarrow}\right)$ remains finite in the TL. PSs are in the present model subspaces spanned by a ground state and its excited energy eigenstates with pseudoparticle overall deviations such that $\sum_{j=1}^{L_{1}}\left|\delta N_{1}^{N F}\left(q_{j}\right)\right| / L \rightarrow 0$ and $\sum_{n=2}^{\infty} \sum_{j=1}^{L_{n}}\left|\delta N_{n}\left(q_{j}\right)\right| / L \rightarrow 0$ as $L \rightarrow \infty$.

From the use of expansions in the deviations $\delta N_{n}\left(q_{j}\right)=N_{n}\left(q_{j}\right)-N_{n}^{0}\left(q_{j}\right)$ in the TBA equations, Eq. (58), and energy eigenvalues, Eq. (63), the excitation energy $\delta E=E_{f}-E_{G S}$ of PS excited states is up to $\mathcal{O}(1 / L)$ order found to be given by,

$$
\delta E=\sum_{n=1}^{\infty} \sum_{j=1}^{L_{n}} \varepsilon_{n}\left(q_{j}\right) \delta N_{n}\left(q_{j}\right)+\frac{1}{L} \sum_{n=1}^{\infty} \sum_{n^{\prime}=1}^{\infty} \sum_{j=1}^{L_{n}} \sum_{j^{\prime}=1}^{L_{n^{\prime}}} \frac{1}{2} f_{n n^{\prime}}\left(q_{j}, q_{j^{\prime}}\right) \delta N_{n}\left(q_{j}\right) \delta N_{n^{\prime}}\left(q_{j^{\prime}}\right) .
$$

This $n$-pseudoparticle energy functional resembles that of the low-energy Fermi liquid. The main difference is that in a Fermi liquid the quasiparticles undergo zero-momentum forward-scattering interactions only at low energies. In contrast, due to the present spin chain integrability, the $n$-pseudoparticle undergo zero-momentum forward-scattering interactions at all energy scales. This is why the energy functional, Eq. (77), applies at all energy scales.

The only restriction to the applicability of the $n$-pseudoparticle energy functional, Eq. (77), is associated with the PS definition. It is thus such that within the TL the deviations $\delta N_{1}^{N F}\left(q_{j}\right)$ and $\delta N_{n}\left(q_{j}\right)$ for $n>1$ involve a finite number of $n$-pseudoparticles. This implies that,

$$
\lim _{L \rightarrow \infty} \frac{\left(\sum_{j=1}^{L_{1}}\left|\delta N_{1}^{N F}\left(q_{j}\right)\right|+\sum_{n=2}^{\infty} \sum_{j=1}^{L_{n}}\left|\delta N_{n}\left(q_{j}\right)\right|\right)}{L} \rightarrow 0 .
$$


The $n$-pseudoparticles introduced in Section 3.3 have internal degrees of freedom. Those refer to a $n$-pairs configuration within which $n=2, \ldots, \infty$ singlet pairs of $2 n$ physical spins $1 / 2$ are bound when $n>1$. For $n=1$ they refer to a single singlet pair. The energy eigenstates $2 \Pi=L-2 S$ paired spins $1 / 2$ that participate in singlet configurations are not free particles. Indeed, they interact through the Hamiltonian first-neighboring exchange interactions, Eq. (51). All their $\Pi=(L-2 S) / 2$ singlet pairs are actually bound within $(n>1)$ or part of $(n=1)$ such states $N=\sum_{n=1}^{\infty} N_{n}$ composite pseudoparticles. Within the corresponding pseudoparticle representation, this refers to the XXX chain physical spins $1 / 2$ rather than to the usual spinons. Within it, the paired physical spins $1 / 2$ exchange interactions. Those are described by the pseudoparticles zero-momentum forward-scattering interactions associated with the $f$ function terms in the energy functional, Eq. (77).

The $M=2 S$ physical unpaired spins $1 / 2$ left over are those participating in the multiplet configurations. They are not part of composite $n$-pseudoparticles and have a free nature. As was discussed in Section 3.5, they singly occupy lattice sites that play the role of empty sites for the $n$-pseudoparticles. Such pseudoparticles move along the lattice with momentum $q_{j}$, upon interchanging position with the unpaired spins. As reported in that section, the spinons often used as elementary excitations of the model zero-field ground state, are associated with the TBA $n=1$ band holes. Within the present representation, an average number $2 S$ of such $N_{1}^{h} \geq 2 S$ holes describe the translational degrees of freedom of the $M=2 S$ physical spins $1 / 2$. This justifies the free fermion nature of the corresponding spinons.

The $n$-pseudoparticle dispersion $\varepsilon_{n}\left(q_{j}\right)$ in Eq. (77) reads,

$$
\varepsilon_{n}\left(q_{j}\right)=\varepsilon_{n}^{0}\left(q_{j}\right)+2 n \mu_{B} h \text { and } \varepsilon_{n}^{0}\left(q_{j}\right)=-\frac{J}{n}\left(1+\cos k_{0}^{n}\left(q_{j}\right)-\int_{-\pi}^{\pi} d k \sin k \bar{\Phi}_{1 n}\left(k, k_{0}^{n}\left(q_{j}\right)\right)\right) .
$$

Here $k_{0}^{n}\left(q_{j}\right)$ denotes the ground-state momentum rapidity $k^{n}\left(q_{j}\right)$. The dressed rapidity phase shifts $\bar{\Phi}_{n n^{\prime}}\left(k, k^{\prime}\right)$ and dressed momentum phase shifts $\Phi_{n n^{\prime}}\left(q_{j}, q_{j^{\prime}}\right)$ in units of $2 \pi$ are defined by the following integral equations and relation,

$$
\begin{aligned}
\bar{\Phi}_{n n^{\prime}}\left(k, k^{\prime}\right) & =\frac{1}{2 \pi} \Theta_{n n^{\prime}}\left(n \tan (k / 2)-n^{\prime} \tan \left(k^{\prime} / 2\right)\right)-\frac{1}{4 \pi} \int_{-Q}^{Q} d k^{\prime \prime} \frac{\Theta_{n 1}^{[1]}\left(n \tan (k / 2)-\tan \left(k^{\prime \prime} / 2\right)\right)}{\cos ^{2}\left(k^{\prime \prime} / 2\right)} \bar{\Phi}_{1 n^{\prime}}\left(k^{\prime \prime}, k^{\prime}\right), \\
\Phi_{n n^{\prime}}\left(q_{j}, q_{j^{\prime}}\right) & =\bar{\Phi}_{n n^{\prime}}\left(k_{0}^{n}\left(q_{j}\right), k_{0}^{n^{\prime}}\left(q_{j^{\prime}}\right)\right),
\end{aligned}
$$

respectively. Here $Q= \pm k_{0}^{1}\left( \pm q_{F}\right)$ and $\Theta_{n n^{\prime}}^{[1]}(x)$ is the derivative of the function $\Theta_{n, n^{\prime}}(x)$, Eq. (B.1) of Appendix B given in Eq. (B.2) of that Appendix.

The $f$ functions in Eq. (77) read,

$$
f_{n n^{\prime}}\left(q_{j}, q_{j^{\prime}}\right)=v_{n}\left(q_{j}\right) 2 \pi \Phi_{n n^{\prime}}\left(q_{j}, q_{j^{\prime}}\right)+v_{n^{\prime}}\left(q_{j^{\prime}}\right) 2 \pi \Phi_{n^{\prime} n}\left(q_{j^{\prime}}, q_{j}\right)+\frac{v}{2 \pi} \sum_{\imath= \pm} 2 \pi \Phi_{1 n}\left(\iota q_{F}, q_{j}\right) 2 \pi \Phi_{1 n^{\prime}}\left(\iota q_{F}, q_{j^{\prime}}\right),
$$

where the group velocities are in the TL given by $v_{n}\left(q_{j}\right)=\left.v_{n}(q)\right|_{q_{j}=q}$ with $v_{n}(q)=d \varepsilon_{n}(q) / d q$. Moreover, $v \equiv v_{1}\left(q_{F}\right)$ is the $n=1$ pseudoparticle Fermi velocity.

When defined in general PSs, the spin- $1 / 2 X X X$ chain is a quantum liquid of $n=1, \ldots, \infty n$-pseudoparticle branches. Such pseudoparticles have residual zero-momentum forward-scattering interactions associated with the term of second order in the deviations in the energy functional, Eq. (77). At $H=0$ the non-Abelian global spin $S U(2)$ symmetry renders gapless the excited energy eigenstates with $n>1 n$-pseudoparticle occupancy. Those are described by groups of real and complex rapidities. Hence the spin dynamical structure factors have contributions from transitions from the $m=0$ ground state to these excited states.

Turning on the magnetic field $H$, drives the system into $m \neq 0$ PSs. For them there emerges an energy gap $\Delta_{s}$ between the $m \neq 0$ ground state and its excited states described by groups of real and complex rapidities. Its minimum value reads $\Delta_{s}^{\min }=\varepsilon_{2}(0)$. For $H>0$ the Hamiltonian term $2 \mu_{B} H \sum_{j=1}^{L} \hat{S}_{j}^{z}$ in Eq. (51) does not commute with the global spin $S U(2)$ symmetry off-diagonal generators. Hence for excitation energy below this energy gap the physics is that of a $U(1)$ symmetry quantum problem. Its states are described only by groups of real rapidities, as in the case of the 1D Lieb-Liniger Bose gas. Consequently, for a finite magnetic field, $H>0$, the model static and low-temperature properties are determined by excitations associated with energy eigenstates with finite $n$-pseudoparticle occupancy $N_{1}=(L-2 S) / 2$ only in the $n=1$ band. The same applies to the leading-order contributions to the longitudinal and transverse spin dynamical structure factors at finite energy scales below the gap $\Delta_{s}$.

The physical quantities considered in the following have the same values both at $H=0$ and in the $H \rightarrow 0$ limit. We thus consider finite magnetic fields in the range $0<H<H_{c}$ below the critical magnetic field for fully polarized ferromagnetism $H_{c}=J / \mu_{B}$. Our following analysis refers to the model in $m \neq 0$ PSs. They are spanned by energy eigenstates with finite $n$-pseudoparticle occupancy only in the $n=1$ band. For simplicity, often the $n=1$ pseudoparticles are called pseudoparticles and their index $n=1$ is omitted from most quantities. For instance, the $n=1$ band is sometimes in the following called pseudoparticle band or simply band. Moreover, $N_{1}=\Pi=(L-2 S) / 2, N_{1}^{h}=M=2 S$, and $L_{1}=(L+2 S) / 2$ for the PSs under consideration.

Within the TL, the set of $j=1, \ldots,(L+2 S) / 2$ momentum values $\left\{q_{j}\right\}$ in the pseudoparticle band may be replaced by a continuum momentum variable, $q \in\left[-k_{F \uparrow}, k_{F \uparrow}\right]$. The set of real rapidities $\Lambda_{j}=\Lambda\left(q_{j}\right)$, Eq. (57) for $n=1$, are then replaced by a rapidity function, $\Lambda=\Lambda(q) \in[-\infty, \infty]$, with $\Lambda\left( \pm k_{F \uparrow}\right)= \pm \infty$. The $m \in[0,1]$ ground states pseudoparticle momentum 
occupancy range is $q \in\left[-k_{F \downarrow}, k_{F \downarrow}\right]$. For $m>0$ such states are thus populated by band holes for $|q| \in\left[k_{F \downarrow}, k_{F \uparrow}\right]$. (The BA band is full for the $m=0$ absolute ground state.)

To second order in the band momentum distribution deviations $\delta N\left(q_{j}\right)=N\left(q_{j}\right)-N^{0}\left(q_{j}\right)$, the energy spectrum functional of the excited states has for the present subspaces the general form given in Eq. (10) with the summations $\sum_{j=1}^{\infty}$ replaced by $\sum_{j=1}^{(L+2 S) / 2}$. This is as given in Eq. (10) for the 1D Lieb-Liniger Bose gas. Furthermore, the energy dispersion in the term of first order in the deviations is now $\varepsilon\left(q_{j}\right) \equiv \varepsilon_{1}\left(q_{j}\right)$, Eq. (79) for $n=1$. The dispersion $\varepsilon^{0}\left(q_{j}\right) \equiv \varepsilon_{1}^{0}\left(q_{j}\right)$ controls the spin density curve as follows,

$$
H(m)=-\left.\frac{\varepsilon^{0}\left(k_{F \downarrow}\right)}{2 \mu_{B}}\right|_{m=1-2 k_{F \downarrow}} .
$$

This applies to the spin density interval $m \in] 0,1]$ and thus to the corresponding magnetic-field range $\left.H \in] 0, H_{c}\right]$.

The energy dispersions $\varepsilon\left(q_{j}\right)$ and $\varepsilon^{0}\left(q_{j}\right)$ have in the $m \rightarrow 0$ and $m \rightarrow 1$ limits the following analytical expressions,

$$
\begin{aligned}
\varepsilon\left(q_{j}\right) & =\varepsilon^{0}\left(q_{j}\right)=-J \frac{\pi}{2} \cos \left(q_{j}\right) \text { for } m \rightarrow 0, \\
\varepsilon\left(q_{j}\right) & =-J\left[\cos \left(q_{j}\right)-1\right] \text { for } m \rightarrow 1, \\
\varepsilon^{0}\left(q_{j}\right) & =-J\left[\cos \left(q_{j}\right)+1\right] \text { for } m \rightarrow 1,
\end{aligned}
$$

respectively. Hence the corresponding group velocity $v(q)=d \varepsilon(q) / d q$ and Fermi velocity $v=v\left(q_{F}\right)$ have the limiting behaviors,

$$
\begin{aligned}
& v\left(q_{j}\right)=J \frac{\pi}{2} \sin \left(q_{j}\right) \text { and } v=J \frac{\pi}{2} \text { for } m \rightarrow 0, \\
& v\left(q_{j}\right)=J \sin \left(q_{j}\right) \text { and } v=0 \text { for } m \rightarrow 1 .
\end{aligned}
$$

Furthermore, in the $m \rightarrow 0$ limit, the dressed phase shift $2 \pi \Phi\left(q_{j}, q_{j^{\prime}}\right) \equiv 2 \pi \Phi_{11}\left(q_{j}, q_{j^{\prime}}\right)$, Eq. (80) for $n=n^{\prime}=1$, has in units of $2 \pi$ at $q_{j}=\iota q_{F}=\iota k_{F \downarrow}$ (where $\iota= \pm$ ) the limiting values,

$$
\Phi\left(\iota k_{F \downarrow}, q_{j}\right)=\frac{\iota}{2 \sqrt{2}} \text { for } q_{j} \neq \iota k_{F \downarrow} \text { and } \Phi\left(\iota k_{F \downarrow}, \iota k_{F \downarrow}\right)=\frac{\iota}{2 \sqrt{2}}(3-2 \sqrt{2}) \text {. }
$$

In the opposite $m \rightarrow 1$ limit it reads in such units,

$$
\Phi\left(q_{j}, q_{j^{\prime}}\right)=\frac{1}{\pi} \arctan \left(\frac{1}{2}\left[\tan \left(\frac{q_{j}}{2}\right)-\tan \left(\frac{q_{j^{\prime}}}{2}\right)\right]\right) .
$$

The $i=0,1$ "renormalized" Fermi velocities $v_{i}$ have the same general expression as for the 1D Lieb-Liniger Bose gas, Eq. (17). (Their specific expressions, Eq. (20), apply though only to that model.) The $i=0$, 1 dressed phase shift parameters $\xi^{i}$ in Eq. (20) have also the same general expression, Eq. (18).

For spin density $m>0$ the energy gap $\Delta_{s}$ between the ground state and the lowest-energy state with $n>1$ band finite $n$-pseudoparticle occupancy is an increasing function of $m$. For low temperatures $T<\Delta_{S} / k_{B}$ the entropy has the form given in Eq. (23) with the summation $\sum_{j=1}^{\infty}$ replaced by $\sum_{j=1}^{(L+2 S) / 2}$. Furthermore, the thermal momentum distribution function deviation $\delta N\left(q_{j}\right)$ has also the same general form as for the 1D Lieb-Liniger Bose gas, Eq. (24). The energy dispersion is however model dependent. The use of the same procedures as for that gas then leads for spin densities not too near $m=1$ to the following low-temperature specific temperature leading order term,

$$
\frac{c_{V}}{L}=\frac{k_{B} \pi}{3 v}\left(k_{B} T\right) .
$$

This is the result also reached by conformal field theory $[45,46]$.

The specific-heat expression, Eq. (87), is valid at very low temperatures $T \ll 2 \mu_{B}\left(H_{c}-H\right) / k_{B}$ for $\left(H_{c}-H\right)>0$. On the one hand, at $H=0$ the low-temperature thermal excitations that contribute to the specific heat expression, Eq. (87), are singlet excited states with $n$-strings of length $n>1$. For zero spin density they refer to gapless branches. On the other hand, for $H>0$ such excited states become gapped. The thermal excitations that contribute to the low-temperature specific heat are then replaced by singlet excited states. They belong to a gapless branch generated by $n=1$ band low-energy and smallmomentum particle-hole processes around that band Fermi points. Such states have no $n$-strings of length $n>1$. For $H>0$ the specific heat, Eq. (87), can be expressed in terms of an effective mass $m^{*}=k_{F \downarrow} / v$ as $c_{V} / L=\left[2 k_{B} m^{*} / 3(1-m)\right]\left(k_{B} T\right)$.

The specific-heat expression obtained for $H>0$ leads in the $H \rightarrow 0$ limit to the correct $H=0$ expression. This is in spite of the $H>0$ and $H=0$ expressions having contributions from the above two different types of gapless excited-state branches. In contrast, the specific-heat expression, Eq. (87), is not valid in the $m \rightarrow 1$ limit. This is because it does not describe properly the crossover to the specific heat exponential regime. Such a regime arises due to the gap $2 \mu_{B}\left(H-H_{c}\right)$ in the excitation spectrum for $H>H_{c}$. Near $H=H_{c}$ the minimum gap for excited energy eigenstates with $n$-strings of length $n>1$ reads $\Delta_{s}^{\min }=3 \mathrm{~J}$. Hence for low temperatures, $T<\Delta_{\mathrm{s}}^{\min } / k_{B}=3 \mathrm{~J} / k_{B}$, the processes contributing to the specific heat only involve $n=1 n$-pseudoparticles and unpaired spins $1 / 2$. At low temperatures the crossover regime involves both the 
above $n=1$ band gapless singlet excited states and across-gap excited energy eigenstates generated by elementary triplet processes.

The term triplet has been used here because the elementary processes under consideration lead to spin deviations $\delta S=1$ and $\delta S=-1$, respectively. Some authors thus associate such elementary processes with creation and annihilation, respectively, of spin-1 magnons. This is mainly an issue of wording. Nonetheless, as mentioned in Section 3.1, within the representation of the spin-1/2 XXX chain in terms of its $L$ physical spins $1 / 2$ the configurations of the excited states under consideration lack the spin-1 magnons as defined for the model on for example the square lattice.

Specifically, within an elementary $\delta S=1$ process a transition from the ground state to an excited state whose minimum gap is $2 \mu_{B}\left(H_{c}-H\right)$ for $H<H_{c}$ occurs. Within it, one singlet pair is broken. As a consequence a deviation $\delta \Pi=-1$ occurs. Here $\Pi$ is the number of singlet pairs, Eq. (64). That elementary process thus corresponds to the annihilation of one $n=1$ $n$-pseudoparticle. It is thus also associated with a deviation $\delta N_{1}=-1$. The two physical spins $1 / 2$ that emerge from the broken singlet configuration join the excited state multiplet configuration. Indeed, two initial-state paired physical spins $1 / 2$ become two final-state unpaired physical spins $1 / 2$. Consistently with the number of unpaired spins $1 / 2$ reading $M=2 S$, the emergence of the two unpaired spins $1 / 2$ leads to a deviation $\delta M=2$. It is behind the spin deviation $\delta S=1$. There is a related deviation, $\delta N_{1}^{h}=2$, in the number of holes in the $n=1$ band. Such two holes describe the translational degrees of freedom of the two unpaired physical spins $1 / 2$ that emerge from the singlet-pair breaking.

Similarly, within an elementary $\delta S=-1$ process a transition from the ground state to an excited state occurs. Its minimum gap reads $2 \mu_{B}\left(H-H_{c}\right)$ for $H>H_{c}$. Two unpaired physical spins $1 / 2$ are annihilated and one singlet pair is created under it. This gives rise to the deviations $\delta \Pi=1, \delta M=-2$, and $\delta S=-1$. Within this inverse elementary process, one $n=1 n$-pseudoparticle is thus created, so that $\delta N_{1}=1$. There is a related deviation $\delta N_{1}^{h}=-2$ in the number of holes in the $n=1$ band.

Such an analysis confirms that the two opposite elementary triplet processes do not correspond to creation and annihilation of one spin-1 magnon, respectively, as defined for the spin-1/2 XXX model on for example the square lattice. Rather, such processes involve the breaking and creation of one singlet pair. Under it two paired physical spins $1 / 2$ are transformed into two unpaired physical spins $1 / 2$ and vice versa, respectively.

The effect of the temperature $T$ smooths the transition to fully polarized ferromagnetism. Therefore, the spin density $m=1$ is only reached as $H \rightarrow \infty$ instead of for $H \rightarrow H_{c}=J / \mu_{B}$ at $T=0$. Nevertheless, at low temperatures the critical magnetic field $H_{c}$ remains a useful reference parameter. To derive the specific heat in the close neighborhood of $H_{c}$, one uses a thermal momentum distribution function deviation of the general form, Eq. (24). In the present case of the spin-1/2 XXX chain, the ground-state distribution in it is $N^{0}(q)=N_{1}^{0}(q)$, Eq. (75). The $n=1$ band energy dispersion used in such a thermal momentum distribution function deviation is that suitable for spin density $m \rightarrow 1$, which reads $\varepsilon(q) \approx q^{2} /\left(2 m^{*}\right)-2 \mu_{B}\left(H_{c}-H\right)$. The effective triplet mass $m^{*}=1 / \mathrm{J}$ in its expression refers to the $m \rightarrow 1$ limit of the above general effective mass $m^{*}=k_{F \downarrow} / v$. The corresponding thermal excited $n=1$ band momentum distribution function controls the density of singlet pairs in the crossover critical regime. It reads,

$$
\frac{\Pi}{L}=\frac{1}{2 \pi} \int_{-\pi}^{\pi} d q N(q)=\frac{1}{2 \pi} \int_{-\pi}^{\pi} d q \frac{1}{1+e^{\varepsilon(q) / k_{B} T}} \approx \frac{\sqrt{2 m^{*} k_{B} T}}{\pi} \int_{0}^{\infty} d x \frac{1}{e^{x^{2}-\frac{2 \mu_{B}\left(H_{C}-H\right)}{k_{B} T}}+1} .
$$

Up to first order in $2 \mu_{B}\left|H_{c}-H\right| / k_{B} T \ll 1$ and yet low temperature, one does not need to account for the temperature dependence of the mass $m^{*}$ in the above energy dispersion.

One uses the thermal momentum distribution function deviation in the energy functional, Eq. (77). We then find that before reaching its maximum magnitude at a field slightly larger than $H_{c}=J / \mu_{B}$, the low-temperature specific heat behaves in a small field window $2 \mu_{B}\left|H-H_{c}\right| \ll k_{B} T$ around $H_{c}=J / \mu_{B}$ as [62],

$$
\frac{c_{V}}{L}=k_{B} c_{0} \sqrt{\frac{m^{*} k_{B} T}{2}}\left(c_{1}+c_{2} \frac{2 \mu_{B}\left(H_{c}-H\right)}{k_{B} T}\right) \text { for } 2 \mu_{B}\left|H-H_{c}\right| \ll k_{B} T .
$$

Here,

$$
c_{0}=\frac{(\sqrt{2}-1)}{4 \pi} ; \quad c_{1}=3 \sqrt{2} \Gamma(3 / 2) \zeta(3 / 2) ; \quad c_{2}=\Gamma(1 / 2) \zeta(1 / 2) .
$$

$\Gamma(x)$ and $\zeta(x)$ are in this equation the usual gamma and Riemann zeta functions, respectively. The specific heat expression, Eq. (89), cannot be derived within conformal-field theory. For larger fields, $H \gg H_{C}+k_{B} T /\left(2 \mu_{B}\right)$, the specific heat vanishes exponentially, $c_{V} / L \propto e^{-2 \mu_{B}\left(H-H_{C}\right) /\left(k_{B} T\right)}$.

In the crossover regime defined by Eq. (89), both the gapless and across-gap channels associated with singlet excitations and excitations generated by elementary spin-triplet processes, respectively, are thermally active. That equation is only valid for a very narrow region around $H_{c}$. In Appendix D it is shown that in the $2 \mu_{B}\left|H-H_{C}\right| \ll k_{B} T$ limit in which that equation is valid it is exactly that obtained by expanding up to first order in $2 \mu_{B}\left|H-H_{c}\right| /\left(k_{B} T\right)$ the general scaling function of the specific heat derived in Ref. [206].

Procedures that resemble those of a Fermi liquid allow as well the derivation of static quantities. For instance, in Appendix E such a type of procedures is used to show that the spin susceptibility, $\chi=2 \mu_{B} /(\partial h(m) / \partial m)$, is fully controlled by the renormalized velocity $v_{0}$. Similarly to Eqs. (17) and (18) for the Bose gas, $v^{i}=v+\frac{1}{2 \pi} \sum_{\iota= \pm}(\iota)^{i} f\left(k_{F \downarrow}, \iota k_{F \downarrow}\right)=v\left(\xi^{i}\right)^{2}$ 

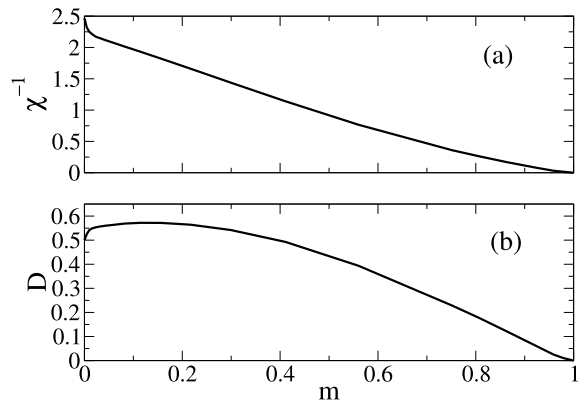

Fig. 6. (a) The inverse spin susceptibility $\chi^{-1}$ and (b) the spin stiffness $D$ given in Eq. (91) as functions of the spin density. Source: From Ref. [62].

and $\xi^{i}=1+\Phi\left(k_{F \downarrow}, k_{F \downarrow}\right)+(-1)^{i} \Phi\left(k_{F \downarrow},-k_{F \downarrow}\right)$ where $i=0,1, v \equiv v_{1}\left(k_{F \downarrow}\right), f \equiv f_{11}$, and $\Phi \equiv \Phi_{11}$. The zero-temperature spin stiffness $D$ in the real part of the spin conductivity Drude peak, $2 \pi D \delta(\omega)$, is in that Appendix shown to be controlled by the renormalized velocity $v_{1} . D>0$ means physically the occurrence of spin ballistic transport.

The spin susceptibility and zero-temperature spin stiffness are in Appendix E found to read,

$$
\chi=\frac{4 \mu_{B}^{2}}{\pi} \frac{1}{v_{0}} \text { and } D=\frac{2 v_{1}}{\pi},
$$

respectively.

In the $m \rightarrow 0$ limit the spin stiffness expression in Eq. (91) recovers the stiffness found in Ref. [177]. The inverse spin susceptibility $\chi^{-1}$ in units of $\mu_{B}=1$ and $J=1$ and spin stiffness $D$ in units of $J=1$ are plotted in Fig. 6(a) and (b), respectively, as a function of the spin density for $m \in[0,1][62]$.

\subsection{The spin-1/2 XXX chain longitudinal and transverse dynamical structure factors in the vicinity of their lower thresholds}

The dynamical structure factors are controlled by matrix elements of spin operators between energy eigenstates. At zero magnetic field this includes states described by groups of real and complex rapidities. Such states have more than two holes in the $n=1$ band. Within the conventional spinon representation they are often called multi-spinon states. An early construction to study the zero-field dynamical structure factor is based on exact results for the simpler XY model, numerical computations on small chains, and known sum rules [114]. It combines rather accurate reproduction of a number of features with its simplicity. It leads to the exact square root singularity at the lower threshold of the spectrum. Such a construction is commonly used in the interpretation of experimental data. However some of the expressions obtained within it are not exact. An example is the functional form at the top of the two $n=1$ band holes continuum, usually called two-spinon continuum.

Mapping the infinite chain onto a relativistic quantum field theory is another successful important scheme [42,43,207]. The connection of the critical exponents of the system with its behavior in a finite volume is achieved by finite-size scaling $[45,46]$. Conformal field theory $[208,209]$ and bosonization allow the calculation of asymptotics of correlation functions [210,211]. This includes known normalizations for the first few leading terms in the operator expansion [212-214]. Many studies of the dynamical structure factors in the XXX chain refer to finite systems. Those rely on numerical diagonalizations [215], evaluation of matrix elements between BA states [114,216,217], and the form-factor method [218]. The latter method specifically relies on determinant representations for matrix elements of local spin operators. They are obtained by solving the quantum inverse problem [219-221]. The form-factor method applies to the spin-1/2 XXZ chain, Eq. (50). It provides the dynamical structure factor over the whole Brillouin zone [196,198].

The isotropic $\Delta=1$ model, Eq. (51), considered here poses the most challenging technical problems for theory. This is because at zero magnetic field the contributions from states described by groups of real and complex rapidities with more than two holes in the $n=1$ band (multi-spinon states) must be accounted for. Indeed, at zero field the energy spectrum of such states is gapless. As shown in Ref. [198], for anisotropy parameter $\Delta \in[0,0.8]$ in Eq. (50) and zero magnetic field nearly the whole integrated spectral weight of the dynamical structure factor stems from excited states with two holes in the $n=1$ band. Those are usually designated by two-spinon BA excitations. Such states are technically simpler to handle. However, as $\Delta$ increases from 0.8 to 1.0 , the excited states with four holes in the $n=1$ band (often called fourspinon excitations) contribute increasingly as $\Delta \rightarrow 1$. This also increases the complexity of the quantum problem under consideration. Specifically, in that limit the contributions to it from the $S=1$ and $S^{z}=0$ excited states with two holes in the $n=1$ band correspond to a relative integrated intensity of $\approx 0.75$ [193]. If in addition one accounts for the contributions from $S=1$ and $S^{z}=0$ excited states with four holes in the $n=1$ band, the total integrated intensity increases to $\approx 0.99$. Nonetheless, all the singularities in the dynamical structure factor are determined by contributions from excited states with two holes in the $n=1$ band. 
The spin dynamical structure factors have been extensively studied in the case of zero magnetic field [114,193-198]. For instance, the square root singularity at the lower threshold exponent $-1 / 2$ can be shown from purely phenomenological considerations to be fixed at zero field by the spin $S U(2)$ symmetry invariance alone [197]. In the following we revisit the longitudinal and transverse spin dynamical structure factors for the less studied case of finite magnetic field. The investigations of Refs. [221,222] on the finite-field dynamical structure factors refer mostly to anisotropy parameter $\Delta<1$ in Eq. (50). The line shape in the vicinity of the dynamical structure factor thresholds was predicted in Ref. [222] to be controlled by momentum dependent exponents. Those have been explicitly obtained for the spin-1/2 XXX chain in Ref. [62].

At finite magnetic field the longitudinal and transverse spin dynamical structure factors are different objects. Contributions to such dynamical factors from transitions from the ground state to excited states described by groups of real and complex rapidities are gapped for finite magnetic field. Such states are populated by $n$-pseudoparticles for which $n>1$. Their energy gap is for the spin density values considered in the following larger than the maximum lower threshold energy. Moreover, except for very small fields $H$, these excitations have nearly vanishing spectral weight. For instance, at $m=0.5$ their contributions correspond to a relative intensity of about $3 \times 10^{-7}$ for anisotropy parameter $\Delta=0.3$ and $4 \times 10^{-7}$ for $\Delta=0.7$ [221]. The estimated relative intensity obtained from the extrapolation of these results to $\Delta=1 \mathrm{spin}-1 / 2 \mathrm{XXX}$ chain is not larger than $10^{-6}$.

For simplicity, our study focuses mainly on the spin density $m>0.15$ range. For it the contribution from excited states populated by $n$-pseudoparticles with $n>1$ is negligible. Their energy gap is actually larger than the maximum lower threshold energy. Hence in the following we limit our analysis to finite-field subspaces spanned by energy eigenstates that are not populated by $n$-pseudoparticles with $n>1$. The studies of Ref. [223] reveal that for the spin density $m<0.15$ range, not considered here, decreasing the magnetic field $H$ increases the amount of the spectral weight in the dynamical structure factor $S^{+-}(k, \omega)$ considered below. Such a weight stems from transitions to excited states populated by both $n=1$ and $n=2 n$-pseudoparticles.

As in the case of the 1D Lieb-Liniger Bose gas, the pseudoparticles can be transformed into pseudofermions. This is achieved by means of a suitable shift of their discrete momentum values. The $n$-pseudofermions have exactly the same internal degrees of freedom as the corresponding $n$-pseudoparticles. Indeed, they differ only in the discrete momentum values $\bar{q}_{j}$ and $q_{j}$, respectively. Those are associated with their center of mass motion. In the following we use the pseudofermion representation and corresponding PDT [62] to study the line shape of the longitudinal and transverse spin dynamical structure factors in the vicinity of their lower thresholds. As in the case of the 1D Bose gas, such a representation is particularly suitable to the study of high-energy dynamical correlation functions.

In the $m \neq 0$ PSs considered in Section 3.6, the 1D Lieb-Liniger Bose gas rapidity expression, Eq. (32), is to be replaced by $\Lambda\left(q_{j}\right)=\Lambda^{0}\left(\bar{q}_{j}\right)$. All quantities and corresponding expressions given in Eqs. (33)-(37) for that model remain valid for the spin-1/2 XXX chain. This holds true though provided that in such expressions the dressed phase shift $2 \pi \Phi\left(q_{j}, q_{j^{\prime}}\right)$ is now the pseudofermion phase shift $2 \pi \Phi\left(q_{j}, q_{j^{\prime}}\right) \equiv 2 \pi \Phi_{11}\left(q_{j}, q_{j^{\prime}}\right)$, Eq. (80) for $n=n^{\prime}=1$. Moreover, the energy dispersion $\varepsilon\left(\bar{q}_{j}\right)$ in Eq. (35) has exactly the same form as $\varepsilon\left(q_{j}\right) \equiv \varepsilon_{1}\left(q_{j}\right)$, Eq. (79) for $n=1$, but with the momentum $q_{j}$ replaced by the corresponding canonical momentum, $\bar{q}_{j}=\bar{q}\left(q_{j}\right)$.

The longitudinal and transverse spin dynamical structure factors can be written as,

$$
S^{a a}(k, \omega)=\sum_{f}\left|\left\langle f\left|\hat{S}_{k}^{a}\right| G S\right\rangle\right|^{2} \delta\left(\omega-\omega^{\tau}(k)\right) .
$$

Here $a=x, y, z$ and $\omega^{\tau}(k)=E_{f}^{\tau}-E_{G S}$ is the excitation energy. Thus $E_{f}^{\tau}$ refers to the energies of the excited states that contribute to the longitudinal $\tau=l$ and transverse $\tau=t$ dynamical structure factors and $E_{G S}$ is the initial ground state energy. Moreover, $\hat{S}_{k}^{a}$ are in Eq. (92) the Fourier transforms of the usual local $a=x, y, z$ spin operators $\hat{S}_{i}^{a}$, respectively.

In the following we often use the classification of Ref. [114], according to which class (i) and class (ii) excitations are (i) non-LWSs and non-HWSs such that $\left|S^{z}\right|<S$ and (ii) LWSs or/and HWSs such that $\left|S^{z}\right|=S$, respectively.

In the case of the longitudinal dynamical structure factor $S^{z z}(k, \omega)$, the exact line shape in the vicinity of its lower thresholds is within the PDT determined by transitions to excited states that are generated from the $H>0$ ground state by high-energy one pseudofermion particle-hole elementary processes. Those conserve the number $L_{-1 / 2}$ of spins $1 / 2$ of projection $-1 / 2$ [62]. The corresponding energy spectrum, $\omega^{l}(k)=\omega^{l}(-k)$, is for spin densities in the interval $\left.\left.m \in\right] 0,1\right]$ in which the subinterval $m \in[0.15,1]$ considered here is contained of the form,

$$
\omega^{l}(k)=-\varepsilon\left(q_{1}\right)+\varepsilon\left(q_{2}\right) \text { for } k=q_{2}-q_{1} \in[0, \pi] .
$$

Here $\varepsilon(q)$ is the energy dispersion, Eq. (79) for $n=1, q_{1} \in\left[-k_{F \downarrow}, k_{F \downarrow}\right]$, and $q_{2} \in\left[k_{F \downarrow}, k_{F \uparrow}\right]$. The longitudinal dynamical structure factor line shape is determined within the PDT by a set of elementary pseudofermion particle-hole processes of momentum $k=\iota(2 \pi / L)$ and energy $\omega \approx \iota v k$ Such processes occur in the vicinity of the two $\iota= \pm$ Fermi points and dress the high-energy one-pseudofermion particle-hole processes.

For the transverse dynamical structure factor,

$$
S^{x x}(k, \omega)=\frac{1}{4}\left[S^{+-}(k, \omega)+S^{-+}(k, \omega)\right],
$$

one must consider the transitions to excited states that determine the line shape in the vicinity of the lower thresholds of both the dynamical structure factors $S^{+-}(k, \omega)$ and $S^{-+}(k, \omega)$, respectively. Indeed, the corresponding transverse dynamical structure factor spectrum $\omega^{t}(k)$, is here expressed as the superposition of the spectra $\omega^{+-}(k)$ and $\omega^{-+}(k)$. 
The spectra $\omega^{ \pm \mp}(k)$ refer to excited states that are generated from the $H>0$ ground state by high-energy elementary processes. Those conserve the number $L_{-1 / 2}$ of spins $1 / 2$ of projection $-1 / 2$. Such states are generated in addition by zeroenergy processes that involve a $\delta L_{-1 / 2}= \pm 1$ deviation. The latter processes lead as well to a related $\delta N_{t}^{F}= \pm 1$ deviation at the $\iota= \pm$ Fermi points and an overall band momentum shift $\delta q_{j}=\mp \iota \pi / L$ [62]. Here $\delta N_{\iota}^{F}$ is the pseudoparticle number deviation at the $\iota= \pm$ Fermi points. The overall zero-energy processes under consideration are a net zero-momentum process. The high-energy elementary processes associated with the spectra $\omega^{+-}(k)$ and $\omega^{-+}(k)$ are in terms of $n=1$ band occupancies, one two-hole elementary processes and one pseudofermion particle-hole elementary processes, respectively.

Hence for spin densities $m \in] 0,1]$ (and thus $m \in[0.15,1]$ ) such spectra read,

$$
\begin{aligned}
& \omega^{+-}(k)=-\varepsilon\left(q_{1}\right)-\varepsilon\left(q_{2}\right) \text { for } k=\pi-q_{1}-q_{2} \in[0, \pi] ; \\
& \omega^{-+}(k)=\varepsilon\left(q_{2}\right)-\varepsilon\left(q_{1}\right) \text { for } k=\pi+q_{2}-q_{1} \in[0, \pi] .
\end{aligned}
$$

Here $q_{1} \in\left[-k_{F \downarrow}, k_{F \downarrow}\right]$ for both spectra, $q_{2} \in\left[-k_{F \uparrow}, k_{F \downarrow}\right]$ for the +- spectrum, and $q_{2} \in\left[-k_{F \downarrow},-k_{F \downarrow}\right]$ for the -+ spectrum. The line shape of the transverse dynamical structure factor is within the PDT also determined by a set of elementary pseudofermion particle-hole processes in the vicinity of the two $\iota= \pm$ Fermi points. Such processes dress the above mentioned high- and zero-energy elementary processes.

A particle (and hole) branch line is a spectral feature that within the PDT is generated by high-energy elementary processes. One pseudofermion (and pseudofermion hole) is under them created outside the Fermi points. Such processes are dressed by pseudofermion particle-hole processes in the vicinity of such points. If the transition to the excited states involves creation or annihilation of other pseudofermions, in the case of a branch line it occurs at the $\iota= \pm$ Fermi points. For both spin densities $m \rightarrow 0$ and $m>0.15$, the lower threshold of $\omega^{l}(k)$ (and $\omega^{t}(k)$ ) coincides with a hole branch line for $k \in\left[0,2 k_{F \downarrow}\right]$ (and $k \in\left[\pi-2 k_{F \downarrow}, \pi\right]$ ) and with a particle branch line for $k \in\left[2 k_{F \downarrow}, \pi\right]$ (and $k \in\left[0, \pi-2 k_{F \downarrow}\right]$ ).

The use of the PDT reveals that the lower threshold singularities of $S^{x x}(k, \omega)$ are those of $S^{-+}(k, \omega)$ near the particle branch line. Near the hole branch line they are those of $S^{+-}(k, \omega)$. Accounting for $\varepsilon\left( \pm k_{F \downarrow}\right)=0$, the longitudinal $S^{z z}(k, \omega)$ and transverse $S^{x x}(k, \omega)$ hole branch lines spectra can be expressed as,

$$
\begin{aligned}
\omega_{h}^{\tau}(k) & =-\varepsilon(q) \text { for } \tau=l, t, \\
k & =k_{F \downarrow}-q \in\left[0,2 k_{F \downarrow}\right] \text { for } \tau=l, \\
k & =\pi-k_{F \downarrow}-q \in\left[\pi-2 k_{F \downarrow}, \pi\right] \text { for } \tau=t .
\end{aligned}
$$

Here $q \in\left[-k_{\digamma \downarrow}, k_{F \downarrow}\right]$. The corresponding particle branch lines spectra read,

$$
\begin{aligned}
\omega_{p}^{\tau}(k) & =\varepsilon(q) \text { for } \tau=l, t, \\
k & =k_{F \downarrow}+q \in\left[2 k_{F \downarrow}, \pi\right] \text { for } \tau=l, \\
k & =\pi-k_{F \downarrow}+q \in\left[0, \pi-2 k_{F \downarrow}\right] \text { for } \tau=t,
\end{aligned}
$$

with $q \in\left[k_{F \downarrow}, k_{F \uparrow}\right]$ and $q \in\left[-k_{F \uparrow},-k_{F \downarrow}\right]$ for the $l$ and $t$ particle branch lines, respectively.

In the present case of the longitudinal and transverse dynamical structure factors, the use of the PDT suitable to the spin-1/2 XXX chain leads to the following high-energy line shape valid for small energy deviations $\left(\omega-\omega^{\tau}(k)\right)>0$ [62],

$$
S^{a a}(k, \omega)=C^{\tau}\left(\omega-\omega^{\tau}(k)\right)^{\xi_{\tau}(k)} \text { for } k \in[0, \pi] \text { where } \xi_{\tau}(k)=-1+\sum_{\imath= \pm} 2 \Delta_{\tau}^{\iota}(q) .
$$

In this equation, $a=z$ for $\tau=l, a=x$ for $\tau=t$, and $C^{\tau}$ is a coefficient whose value remains unchanged in the range of small energy deviations $\left(\omega-\omega^{\tau}(k)\right)>0$ for which the present expression is valid. The $q$ ranges are related to those of the physical momentum $k$ as $q=k_{F \downarrow}-k$ and $q=-k_{F \downarrow}+k$ for the $S^{z z}(k, \omega)$ hole and particle branch lines, respectively. For the hole and particle branch line of $S^{x x}(k, \omega)$ one has that $q=\pi-k_{F \downarrow}-k$ and $q=-\pi+k_{F \downarrow}+k$, respectively. Moreover, the functionals $2 \Delta_{\tau}^{\iota}(q)$ in Eq. (98) are the square of the pseudofermion $\iota= \pm$ Fermi points deviations $\left(\delta \bar{q}_{F}^{\iota} /(2 \pi / L)\right)^{2}$, Eq. (37), specific to the present excitations. Those are given by [62],

$$
2 \Delta_{l}^{\iota}(q)=\left(\frac{\left(\xi^{1}\right)^{2}-\iota c}{2 \xi^{1}}+c \Phi\left(\iota k_{F \downarrow}, q\right)\right)^{2} \text { and } 2 \Delta_{t}^{\iota}(q)=\left(\frac{\xi^{1}}{2}+c \Phi\left(\iota k_{F \downarrow}, q\right)\right)^{2},
$$

respectively. Here $q \in\left[-k_{F \downarrow}, k_{F \downarrow}\right]$ for $c=-1$ and $\tau=l, t, q \in\left[k_{F \downarrow}, k_{F \uparrow}\right]$ for $c=1$ and $\tau=l$, and $q \in\left[-k_{F \uparrow},-k_{F \downarrow}\right]$ for $c=1$ and $\tau=t$. The band momentum $q$ is related to the physical excitation momentum $k$ as given in Eqs. (96) and (97).

The longitudinal spectrum $\omega^{l}(k)$, Eq. (93), and the transverse spectrum $\omega^{t}(k)$ that results from combination of the spectra $\omega^{+-}(k)$ and $\omega^{-+}(k)$, Eq. (95), along with the corresponding exponents $\xi^{l}(k)$ and $\xi^{t}(k)$, respectively, given in Eq. (98), are plotted in Fig. 7 for spin densities (a) $m=0.16$ and (b) $m=0.25$. In Fig. 8 they are plotted for (a) $m=0.30$ and (b) $m=0.50$ and in Fig. 9 for (a) $m=0.75$ and (b) $m=0.99$. On the one hand, the exponent $\xi^{l}(k)$ is negative for $k>0$ at any $m$ value. On the other hand, the exponent $\xi^{t}(k)$ is negative for a $m$-dependent range $k \in\left[k_{t}, \pi\right]$. Here $k_{t}$ increases from $k_{t}=0$ for $m \rightarrow 0$ 

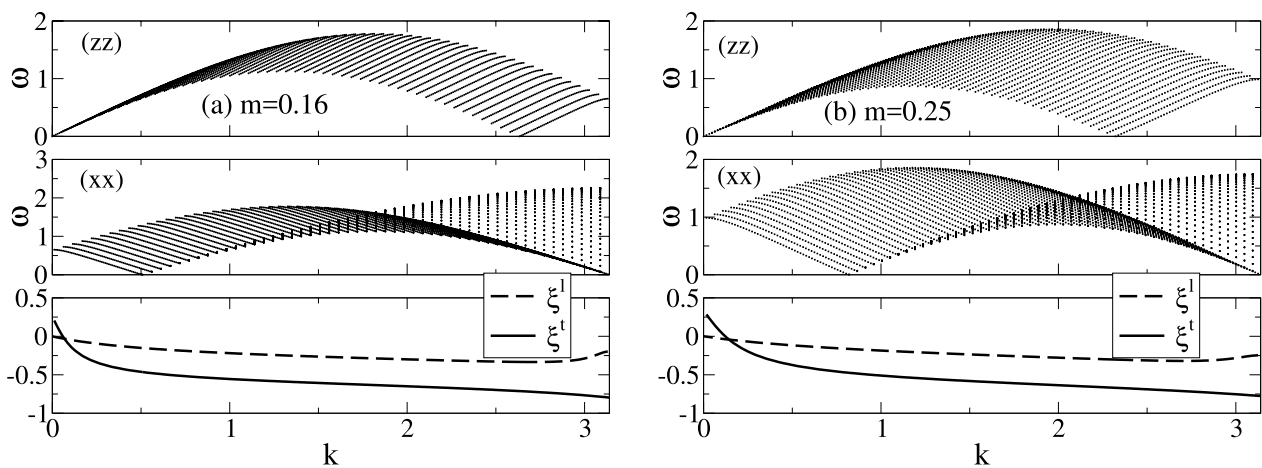

Fig. 7. Two upper panels of each sub-figure (a) and (b): The spectra $\omega^{l}(k)$ and $\omega^{t}(k)$ for spin densities (a) $m=0.16$ and (b) $m=0.25$. Lower panels: The exponents $\xi^{l}(k)$ and $\xi^{t}(k)$, Eq. (98), that control the singularities in the vicinity of the lower thresholds of the spectra also plotted here as a function of $k \in[0, \pi]$. Source: From Ref. [62].
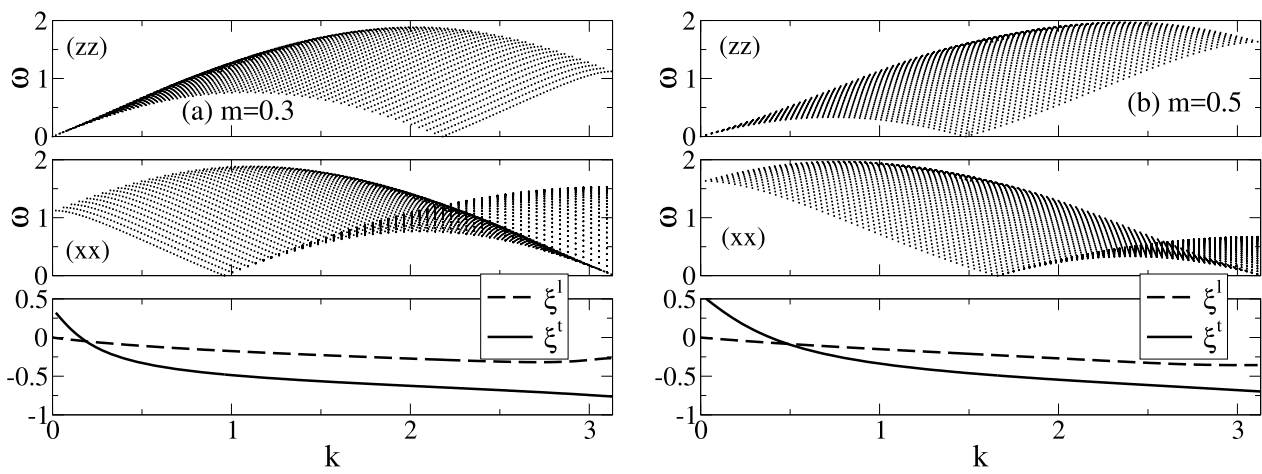

Fig. 8. The same quantities as in Fig. 7 for spin densities (a) $m=0.30$ and (b) $m=0.50$. Source: From Ref. [62].

to,

$$
k_{t}=-2 \arctan \left(\frac{1}{2} \tan \left(\frac{\pi}{\sqrt{2}}\right)\right) \approx 0.37 \pi,
$$

for $m \rightarrow 1$. The latter limit refers to Fig. 9(b).

In the $m \rightarrow 0$ limit, the spectra $\omega^{l}(k)$, Eq. (93), and $\omega^{+-}(k)$, Eq. (95), plotted in Figs. 7-9 reduce to their lower thresholds. At finite $m$, the thresholds of these two spectra correspond to different $(k, \omega)$-plane lines. As $m \rightarrow 0$ they become the same $(k, \omega)$-plane line. For finite $m$ values the lower threshold of the spectrum $\omega^{+-}(k)$, Eq. (95), coincides with that of $\omega^{-+}(k)$ for $k \in\left[\pi-2 k_{F \downarrow}, \pi\right]$. For $k \in\left[0, \pi-2 k_{F \downarrow}\right]$ it does not exist. In the $m \rightarrow 0$ limit the lower threshold of the spectrum $\omega^{+-}(k)$ extends to the whole $k \in[0, \pi]$ range. In that limit it coincides with those of $\omega^{l}(k)$ and $\omega^{-+}(k)$. However, in contrast to the latter spectra, $\omega^{+-}(k)$ does not reduce in that limit to its lower threshold. The spectrum of the class (ii) two-hole excitations described by groups of real and complex rapidities is gapped for $m>0$. However, in the $m \rightarrow 0$ limit it becomes gapless and degenerate with that of $\omega^{-+}(k)$.

For the ranges of the momentum $k$ for which the exponent $\xi_{\tau}(k)$ is negative, there are lower threshold singularity cusps in $S^{a a}(k, \omega)$. Eq. (98). Those are detectable as large intensity peaks in experiments. Hence analysis of Figs. 7-9 provides valuable information on the $k$ ranges for which there are singularities in the lower thresholds of the dynamical structure factors $S^{z z}(k, \omega)$ and $S^{x x}(k, \omega)=S^{y y}(k, \omega)$.

In the $m \rightarrow 0$ limit, both the $\tau=l, t$ lower thresholds $\omega_{L}^{\tau}(k)$ coincide with the hole branch line for all $k$ values. In that limit the corresponding exponents $\xi_{\tau}(k)$ are given by $\xi_{\tau}(k)=-1 / 2$ for all $k$ values, i.e.,

$$
S^{z z}(k, \omega)=S^{x x}(k, \omega)=C^{0}\left(\omega-\omega^{0}(k)\right)^{-1 / 2},
$$

for $m \rightarrow 0$ and $k \in[0, \pi]$. Here $C^{0}=C^{l}=C^{t}$ and the lower thresholds $\omega^{l}(k)=\omega^{t}(k)=\omega^{0}(k)$ coincide with that of the $m=0$ two-hole spectrum. Consistently, $\xi_{\tau}(k)=-1 / 2$ is also the value of the known exponent that controls the line shape in the vicinity of the lower threshold of the latter spectrum [193-198]. 

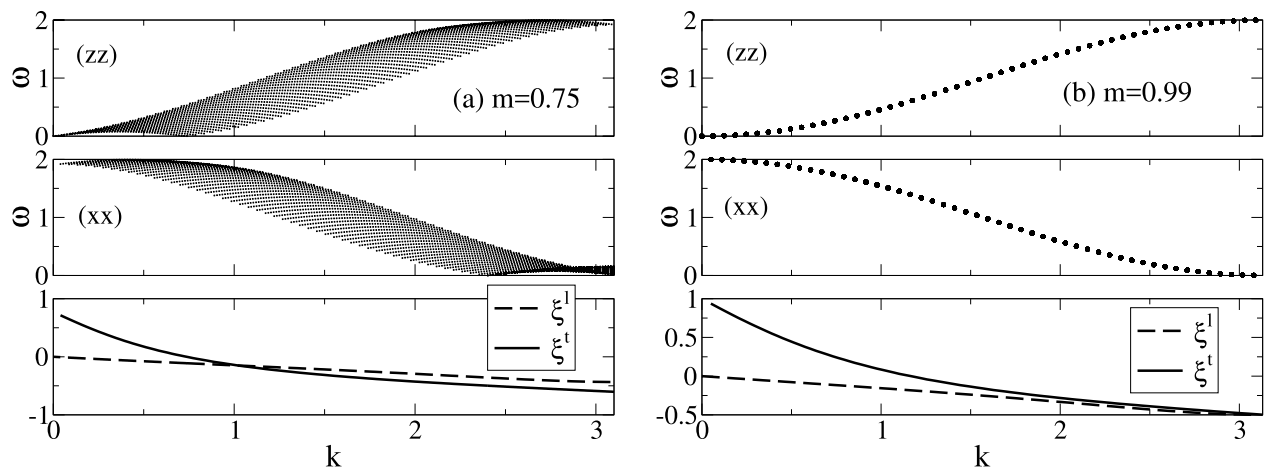

Fig. 9. The same quantities as in Figs. 7 and 8 for spin densities (a) $m=0.75$ and (b) $m=0.99$. Note that the spin density $m=0.99$ approaches the $m \rightarrow 1$ limit within which the spectra $\omega^{l}(k)$ and $\omega^{t}(k)$ become a single line.

Source: From Ref. [62]

In the opposite limit, $m \rightarrow 1$, the lower thresholds $\omega^{\tau}(k)$ coincide with the particle branch line for all $k$ values. The corresponding $\tau=l, t$ exponents are given by,

$$
\xi^{l}(k)=2 \Phi(0, k)[1+\Phi(0, k)] \text { and } \xi^{t}(k)=-1 / 2+2 \Phi(0, k-\pi)[1+\Phi(0, k-\pi)] .
$$

Here the phase shift in units of $2 \pi$ is a particular case of that given in Eq. (86). It reads,

$$
\Phi(0, q)=-\frac{1}{\pi} \arctan \left(\frac{1}{2} \tan \left(\frac{q}{2}\right)\right) .
$$

In this limit, $\xi^{l}(k)=0$ and $\xi^{t}(k)=1$ for $k \rightarrow 0$. The values decrease to $\xi^{l}(k)=-1 / 2$ and $\xi^{t}(k)=-1 / 2$ for $k \rightarrow \pi$. The corresponding $m \rightarrow 1$ behaviors refer to a small but finite $L_{-1 / 2} / L$ ratio. Using the expression for the exponent $\xi^{t}(k)$ in Eq. (102) leads straightforwardly to the value of the momentum $k_{t}$ shown in Eq. (100), which is reached in the $m \rightarrow 1$ limit.

Note though that $S^{z z}(k, \omega) \rightarrow 0$ as $H \rightarrow H_{c}$ in the TL $[62,114]$. The two-component $S^{x x}(k, \omega)$ and $S^{z z}(k, \omega)$ dynamical structure factor is then dominated by $S^{x x}(k, \omega)$. At $H=H_{c}$, the expression given in Eq. (98) is replaced by a $\delta$-function like distribution,

$$
S^{x x}(k, \omega)=\frac{\pi}{2} \delta(\omega-J(1+\cos k)) \text { for } k \in[0, \pi],
$$

for $\alpha \alpha=x x$ and by $S^{z z}(k, \omega)=0$ for $\alpha \alpha=z z$.

The exponent $\xi_{\tau}(k)$ given in Eq. (98) does not apply near the $\omega=0$ lower threshold $(k, \omega)$-plane soft modes. Examples are $\left(k_{0}^{\tau}, 0\right)$ where $k_{0}^{l}=2 k_{F \downarrow}$ and $k_{0}^{t}=\pi-2 k_{F \downarrow}$. In this case the PDT reaches the same results as conformal-field theory [45,46]. Indeed, near them the two $\iota= \pm$ functionals, Eq. (194), become the conformal dimensions of the $\iota= \pm$ fields [181] given by $2 \Delta_{l}^{\iota}=\left(\xi^{1}\right)^{2}$ and $2 \Delta_{t}^{\iota}=\left( \pm \iota /\left(2 \xi^{1}\right)-\xi^{1}\right)^{2}$. The PDT provides the corresponding low-energy $S^{a a}(k, \omega)$ behavior [62,181]. It is the same as that obtainable from conformal-field theory [45,46,176,224,225].

An experimental possibility is the potential observation of the theoretically predicted dynamical structure factors peaks in inelastic neutron scattering experiments on actual spin-chain compounds. The dynamical structure factors $S^{z z}(k, \omega)$ and $S^{x x}(k, \omega)$ may be investigated separately in $H>0$ experiments on spin-chain compounds by using a carefully oriented crystal. If the crystal is missoriented, or if a micro crystalline sample is used, the $S^{z z}(k, \omega)$ and $S^{x x}(k, \omega)$ spectral features should appear superimposed. Such superimposition changes the excitations lower thresholds. It leads in addition to the broadening of the singularities, Eq. (98). However, this does not occur at $H=0$, since $S^{z z}(k, \omega)=S^{x x}(k, \omega)$.

These two different situations are clearly seen in the magnetic scattering intensity measured at zero- and finite-field inelastic neutron scattering experiments of Ref. [136], respectively, on $\mathrm{Cu}\left(\mathrm{C}_{4} \mathrm{H}_{4} \mathrm{~N}_{2}\right)\left(\mathrm{NO}_{3}\right)_{2}$. On the one hand, in Figs. 2(a)-(c) of that reference the theoretically predicted sharp cusps at zero-field, Eq. (101), are clearly seen at different $k$ values. On the other hand, the $S^{z z}(k, \omega) \neq S^{x x}(k, \omega)$ spectral features appear superimposed in the finite-field Figs. 2(d)-(f) of that reference. Therefore, only at $k \approx \pi$ is the theoretically predicted sharp cusp clearly visible.

More demanding $H>0$ experiments with a carefully oriented crystal to be carried out on $\mathrm{Cu}\left(\mathrm{C}_{4} \mathrm{H}_{4} \mathrm{~N}_{2}\right)\left(\mathrm{NO}_{3}\right)_{2}$ and other spin-chain compounds should yielding separately $S^{z z}(k, \omega)$ and $S^{x x}(k, \omega)$. The corresponding magnetic scattering intensities are expected to display the cusp singularities found theoretically reported here.

3.8. Outlook of the relation between the model physical spins-1/2 and the $n$ pseudoparticles/n pseudofermions and corresponding $n$-band holes

The spin-1/2 XXX chain non-Abelian global spin $S U(2)$ symmetry has direct effects on its degrees of freedom. It gives rise to a type of solutions of the BA equation, Eq. (58), in terms of groups of both real and complex rapidities. The latter do not exist for the simpler $U(1)$ symmetry 1D Lieb-Liniger Bose gas. 
For each energy and momentum eigenstate, out of the model physical $L$ spins $1 / 2$, there are $M=2 S$ unpaired spins that contribute to its multiplet configuration. There are in addition $2 \Pi=L-2 S$ paired spins that are bound within $\Pi \equiv(L-2 S) / 2$ singlet pairs. The degrees of freedom of such singlet pairs are distributed over a set $\left\{N_{n}\right\}$ of $n$-pairs configurations. Here $N_{n}$ denotes the number of $n$-pairs configurations within which $n=1, \ldots, \infty$ singlet pairs are bound. The $n=1$ pair configurations contain a single singlet pair that remains unbound.

As in the case of the 1D Lieb-Liniger Bose gas, the problem is non-perturbative. Indeed, a spin-flip decays into a collective excitation that involves all pseudoparticles/pseudofermions. This follows from the boundary conditions, Eqs. (55) and (60). In contrast to the pseudoparticles of that Bose gas, for the spin-chain all $n$-pseudoparticles have internal degrees of freedom. Those are associated with such composite pseudoparticles $n$-pairs configurations and the corresponding $n=1, \ldots, \infty$ singlet pairs that for $n>1$ are bound within them. The $n$-pseudofermions have exactly the same internal degrees of freedom as the $n$-pseudoparticles. They differ from them in the discrete momentum values $\bar{q}_{j}$ and $q_{j}$, respectively. Those are associated with the translational degrees of freedom center of mass motion.

On the one hand, the $\Pi=(L-2 S) / 2$ singlet pairs that contain the $2 \Pi=L-2 S$ physical paired spins $1 / 2$ are bound within the $N=\sum_{n} N_{n}$ pseudoparticles/pseudofermions. This is consistent with the sum rule $\Pi=\sum_{n} 2 n N_{n}$. On the other hand, the translational degrees of freedom of the $M=2 S$ physical unpaired spins are on average described by $2 S$ holes out of the $N_{n}^{h}=2 S+N_{n}^{h, 0}$ holes in each $n$-band for which $N_{n}>0$. The additional $N_{n}^{h, 0}$ holes occurring in states described by groups of real and complex rapidities have a specific goal. It is to ensure that the number of TBA energy and momentum eigenstates that span each fixed-S subspace, Eq. (C.2) of Appendix C, exactly equals the corresponding number of spin $S U$ (2) state representations, Eq. (C.1) of that Appendix. The relation of the usual spinon representation for the model in welldefined subspaces to the more general $n$-bands hole representation for the spin- $1 / 2 X X X$ chain in its full Hilbert space has been discussed and clarified.

Within the $n$-pseudoparticle/n-pseudofermion representations the spin-chain physics simplifies. Within them both the static and dynamical properties are controlled by pseudofermion scattering and corresponding phase shifts. A further simplification occurs for the model in $m \neq 0$ PSs for which there is an energy gap $\Delta_{s}$ between the $m \neq 0$ ground state and its excited states described by groups of real and complex rapidities. For excitation energy below this gap, the physics is that of a $U(1)$ symmetry quantum problem. Its states are described only by groups of real rapidities, as in the case of the 1D Lieb-Liniger Bose gas. Specifically, it is a quantum liquid of $n=1$ pair composite pseudoparticles, which play the same role as the pseudoparticles of that simpler gas.

\section{The 1D Hubbard model: emergent fractionalized particles from rotated electrons}

The 1D Hubbard model is an integrable many-body problem that in spite of being more complex than the 1D Lieb-Liniger Bose gas and spin-1/2 XXX chain has some common properties with both such systems. In this section, an introductory short summary of the 1D Hubbard model development since its coordinate BA solution by Lieb and Wu in 1968 [69,70] is presented. In addition, a uniquely defined unitary operator that transforms electrons into rotated electrons for $u=U / 4 t>0$ is considered. Such rotated electrons have the same charge and spin 1/2 as the electrons. Only their lattice occupancy configurations and corresponding lattice degrees of freedom differ from those of the electrons. Three basic fractionalized particles are introduced. They naturally emerge from the rotated electrons degrees of freedom separation. The relation of the model fractionalized particles and related exotic composite particles to the electrons is clarified.

\subsection{The 1D Hubbard model: a short summary of its development}

Upon the 1D Hubbard model solution in 1968 by the coordinate BA [69,70], its ground state energy was derived. Moreover, the pioneering study reported in Ref. [69] revealed that the model undergoes a Mott metal-insulator transition at density $n_{e}=N_{e} / L=1$ whose corresponding critical onsite interaction is $U=0$. Following that solution, the ground state properties [226-228] and the excitation spectrum [71,74,118,119,229-231] were studied by several authors. This applies as well to the preliminary version of the model pseudoparticle representation of the BA solution reviewed here [89-102].

In 1972 the TBA and corresponding ideal strings have been proposed in Ref. [73] for the 1D Hubbard model. This has allowed the study of the model thermodynamic properties [232,233]. The energy spectra of its elementary excitations can be obtained from the TBA equations in the zero temperature limit [132]. As in the case of the spin-1/2 XXX chain, the use of the TBA has in the TL extended the number of pseudoparticle branches from two to infinite. This ensures that their occupancy configurations generate all the model $4^{L}$ energy and momentum eigenstates [99,103-105].

An important property of the 1D Hubbard model is that its spectrum becomes conformally invariant in the low-energy limit. The corresponding finite-size corrections were obtained in Refs. [234,235]. The relation between the finite-size spectrum and the asymptotic behavior of correlation functions was used to calculate the critical exponents of the model general two-point correlation functions [224,225]. The corresponding conformal dimensions have been expressed in terms of dressed phase shifts associated with the pseudoparticle representation [90,91].

The conformal approach is not applicable to the zero-temperature model Mott insulating phase at half filling. In the small- $U$ and scaling limits, dynamical correlation functions at low energies [236-239] can though be computed relying on methods of integrable quantum field theory [240-242]. Moreover, at half-filling and zero spin density the 1D Hubbard model TBA dressed phase shifts and corresponding $S$-matrices have been associated with particles called holon, antiholon, 
and spinon. The holon and antiholon have zero spin and charge $+e$ and $-e$, respectively. The spinon has been inherently constructed to have no charge and to have spin $1 / 2[66,67]$. The model $S O(4)$ symmetry group state representations were identified with occupancy configurations of such particles.

More recently it was found in Ref. [147] that for $u \neq 0$ the 1D Hubbard model global symmetry is actually larger than $S O(4)$ and given by $[S O(4) \otimes U(1)] / Z_{2}$. (This applies as well to the model on any bipartite lattice.) As further discussed below, in the case of the model full Hilbert space all energy eigenstates can be generated by pseudoparticle occupancy configurations. Those refer to the state representations of the model $[S O(4) \otimes U(1)] / Z_{2}$ symmetry group.

The wave functions of the energy eigenstates can be extracted from the coordinate BA solution. An explicit representation for the wave functions was given in Ref. [118]. The corresponding energy and momentum eigenstates are either LWSs or HWSs $[243,244]$ with respect to the model Hamiltonian $S O(4)$ symmetry in $[S O(4) \otimes U(1)] / Z_{2}[143-146]$. The non-LWSs can be generated from the LWSs, which confirmed the quantum problem completeness [245].

The first steps to obtain the BA solution of the 1D Hubbard model by the quantum inverse scattering method were made in Refs. [120-122]. The model Hamiltonian was mapped under a Jordan-Wigner transformation into a spin Hamiltonian. It commutes with the transfer matrix of a related covering vertex model [120]. The $R$-matrix of the spin model was also derived [121,122]. Alternative derivations were carried out by several authors [123-125]. The $R$-matrix was later shown to satisfy the Yang-Baxter equation [126].

An algebraic BA having as starting point the results of Refs. [120-122] was afterwards constructed in Refs. [127,128] for the 1D Hubbard model. Consistently with the model $[S O(4) \otimes U(1)] / Z_{2}$ symmetry, the corresponding spin and charge monodromy matrices were found to have different $A B C D$ and $A B C D F$ forms, respectively. Those are associated with the spin $S U(2)$ and charge $U(2)=S U(2) \otimes U(1)$ symmetries, respectively [128]. The latter matrix is larger than the former and involves more fields [128]. If the model global symmetry was only $S O(4)=[S U(2) \otimes S U(2)] / Z_{2}$, the charge and spin monodromy matrices would have the same traditional $A B C D$ form, which is that of the spin- $1 / 2 X X X$ chain [78]. The expressions for the eigenvalues of the transfer matrix of the two-dimensional statistical covering model were obtained. That problem was also addressed in Ref. [129].

The algebraic BA introduced in Refs. [127,128] allowed the quantum transfer matrix approach to the thermodynamics of the 1D Hubbard model [246]. Within it, the thermodynamic quantities and correlation lengths can be calculated numerically for finite temperatures [247,248]. The 1D Hubbard model Hamiltonian was found in the TL to be invariant under the direct sum of two $Y(s l(2))$ Yangians [249]. The relation of these Yangians to the above $R$-matrix and the implications of one of these Yangians for the structure of the bare excitations was later clarified [250,251].

In the $u \rightarrow \infty$ limit the dynamical correlation functions can be computed at zero temperature for all energy scales relying on the simplified form that the BA equations acquire. This was achieved by a combination of analytical and numerical techniques for the whole range of electronic densities [150-158,160,161]. In the case of the one-electron spectral function studies of Refs. [156-158], the method relies on the spinless-fermion phase shifts imposed by XXX chain physical spins $1 / 2$. Such elementary objects naturally arise from the zero spin density and $u \rightarrow \infty$ electron wave-function factorization $[118,119,150]$. A related PDT $[13,51,58,59,63,64]$ relying on a representation of the model BA solution in terms of the pseudofermions generated by a unitary transformation from the corresponding pseudoparticles considered in Ref. [105] was introduced in Ref. [51]. It is an extension of the $u \rightarrow \infty$ method of Refs. [156-158] to the whole $u>0$ range of the 1D Hubbard model.

After the PDT of the 1D Hubbard model was introduced, the MQIM methods have been developed to also tackle the high-energy physics of both integrable and non-integrable 1D correlated quantum problems, beyond the low-energy TLL limit $[52,53,56,57]$. In the case of the 1D Hubbard model, the MQIM reaches the same results as the PDT. For instance, the momentum, electronic density, and on-site repulsion $u>0$ dependence of the exponents that control the line shape of the one-electron spectral function of the model at zero magnetic field calculated in Refs. [54,55], in the framework of the MQIM using input from the BA solution, is exactly the same as that obtained previously by the use of the PDT.

\subsection{The Hubbard model and the infinite choices of rotated electrons}

The Hubbard model in a chemical potential $\mu$ and magnetic field $H$ under periodic boundary conditions on a 1D lattice with an even number $L \rightarrow \infty$ of sites is given by,

$$
\hat{H}=t \hat{T}+U \hat{V}_{D}+2 \mu \hat{S}_{\eta}^{z}+2 \mu_{B} H \hat{S}_{s}^{z} .
$$

Here,

$$
\hat{T}=-\sum_{\sigma=\uparrow, \downarrow} \sum_{j=1}^{L}\left(c_{j, \sigma}^{\dagger} c_{j+1, \sigma}+c_{j+1, \sigma}^{\dagger} c_{j, \sigma}\right) \text { and } \hat{V}_{D}=\sum_{j=1}^{L} \hat{\rho}_{j, \uparrow} \hat{\rho}_{j, \downarrow} \text { with } \hat{\rho}_{j, \sigma}=c_{j, \sigma}^{\dagger} c_{j, \sigma}-1 / 2,
$$

are the kinetic-energy operator in units of $t$ and the electron onsite repulsion operator in units of $U$, respectively, and

$$
\hat{S}_{\eta}^{z}=-\frac{1}{2}\left(L-\hat{N}_{e}\right) \text { and } \hat{S}_{s}^{z}=-\frac{1}{2}\left(\hat{N}_{e \uparrow}-\hat{N}_{e \downarrow}\right)
$$


are the diagonal generators of the global $\eta$-spin and spin $S U(2)$ symmetry algebras, respectively. Moreover, in Eqs. (105) and (106) the operator $c_{j, \sigma}^{\dagger}$ (and $c_{j, \sigma}$ ) creates (and annihilates) a spin-projection $\sigma=\uparrow, \downarrow$ electron at lattice site $j=1, \ldots, L$. The electron number operators read $\hat{N}_{e}=\sum_{\sigma=\uparrow, \downarrow} \hat{N}_{e \sigma}$ and $\hat{N}_{e \sigma}=\sum_{j=1}^{L} \hat{N}_{e, j, \sigma}$.

The $\sigma$ electronic momentum distribution operator is given by $\hat{N}_{e \sigma}(k)=c_{k, \sigma}^{\dagger} c_{k, \sigma}$ where $c_{k, \sigma}^{\dagger}$ (and $c_{k, \sigma}$ ) creates (and annihilates) a $\sigma$ electron of momentum $k$. Its $z$-component $\eta$-spin/charge current operator in units of electronic charge $e$ $(\alpha=\eta)$ and $z$-component spin current operator in units of spin $1 / 2(\alpha=s)$ read,

$$
\hat{J}_{\alpha}^{z}=-i 2 t \sum_{\sigma} \sum_{j=1}^{L}(\sigma)^{\delta_{\alpha, s}}\left(c_{j, \sigma}^{\dagger} c_{j+1, \sigma}-c_{j+1, \sigma}^{\dagger} c_{j, \sigma}\right) \text { where } \alpha=\eta, s .
$$

It is here considered in $(\sigma)^{\delta_{\alpha, s}}$ that $\sigma=+1$ and $\sigma=-1$ for $\uparrow$ and $\downarrow$, respectively. These $\eta$-spin/charge and spin current operators are sometimes called in this paper $\alpha=\eta$ and $\alpha=s$ current operators, respectively.

The $4^{L}$ energy and momentum eigenstates of the 1D Hubbard model for $u>0$ can be generated by the independent occupancy configurations of three basic fractionalized particles. They are associated with the two $S U(2)$ symmetries and the $c$-lattice $U(1)$ symmetry, respectively, in the model global $[S U(2) \otimes S U(2) \otimes U(1)] / Z_{2}^{2}$ symmetry mentioned in Sections 1 and 4.1.

The origin of the $u>0$ global $[S U(2) \otimes S U(2) \otimes U(1)] / Z_{2}^{2}$ symmetry is a local gauge $S U(2) \otimes S U(2) \otimes U(1)$ symmetry of the $U>0$ Hamiltonian $t=0$ term first identified in Ref. [252]. At $U=0$ and $t \neq 0$ that local gauge symmetry is unrelated to the model global $S O(4) \otimes Z_{2}$ symmetry. The $1 / Z_{2}^{2}$ factor in the $u>0$ model global symmetry refers to the number $4^{L}$ of its independent representations being four times smaller than the dimension $4^{L+1}$ of the group $S U(2) \otimes S U(2) \otimes U(1)$. In contrast, the factor $Z_{2}$ in the $U=0$ model global $S O(4) \otimes Z_{2}$ symmetry corresponds to a discretely generated symmetry associated with a well-known transformation that exchanges spin and $\eta$-spin. It is an exact symmetry of the $U=0$ and $t \neq 0$ Hamiltonian. However, it changes the sign of $U$ when $U \neq 0$.

The $c$-lattice $U(1)$ symmetry beyond $S O(4)$ found in Ref. [147], which does not exist at $U=0$, emerges at any arbitrarily small finite- $U$ value. The related $U>0$ and $t=0$ local gauge $S U(2) \otimes S U(2) \otimes U(1)$ symmetry becomes for finite $U$ and $t$ a group of permissible unitary transformations. The corresponding local $U(1)$ canonical transformation is not the ordinary gauge $U(1)$ subgroup of electromagnetism. It is rather a "nonlinear" transformation [252]. The $c$-lattice $U(1)$ symmetry has direct effects on the $u>0$ model BA solution structure. Its state representations are generated by occupancy configurations of a specific BA quantum-number branch.

For finite values of chemical-potential $\mu$ and magnetic field $H$ the corresponding operator terms in the Hamiltonian, Eq. (105), lower the model global symmetry. However, such terms commute with that Hamiltonian. Therefore, for finite $u$ and all values of the electronic density $n_{e}$ and spin density $m$, the quantum-numbers occupancy configurations that generate all the model energy eigenstates from the electron or hole vacuum are in one-to-one correspondence to a set of independent state representations of the $\mu=H=0$ model Hamiltonian global symmetry algebra. Hence, for all electronic density $n_{e}$ and spin density $m$ values, that global symmetry algebra fully determines the finite- $u$ energy eigenstates spectrum structure. Furthermore, the number of the model non-Abelian global symmetry independent state representations exactly equals the Hilbert-space dimension, $4^{L}[147]$.

The LWSs and HWSs of the $\eta$-spin and spin $S U(2)$ symmetry algebras have numbers $S_{\alpha}=-S_{\alpha}^{z}$ and $S_{\alpha}=S_{\alpha}^{z}$, respectively, where $\alpha=\eta$ and $\alpha=s$, respectively. As in the case of the spin-1/2XXX chain, in this review the LWS formulation of 1D Hubbard model BA solution is used. Here $S_{\eta}$ is the states $\eta$-spin, $S_{s}$ their spin, and $S_{\eta}^{z}=-\left(L-N_{e}\right) / 2$ and $S_{s}^{z}=-\left(N_{e \uparrow}-N_{e \downarrow}\right) / 2$ are the corresponding projections, respectively. The latter are the eigenvalues of the two $S U(2)$ algebras diagonal generators, Eq. (107). The LWSs of such algebras considered in our studies are energy and momentum eigenstates. Hence, as in the case of the spin-1/2 XXX chain, they are here called LWSs. Such LWSs have electronic densities $n_{e}$ and spin densities $m$ in the ranges $n_{e} \in[0,1]$ and $m \in\left[0, n_{e}\right]$, respectively.

Let $\left\{\left|l_{\mathrm{r}}, l_{\eta s}, u\right\rangle\right\}$ be the complete set of $4^{L}$ energy and momentum eigenstates of the Hamiltonian $\hat{H}$, Eq. (105), associated with the BA solution for $u>0$. The LWSs of both $S U(2)$ symmetry algebras are here denoted by $\left|l_{\mathrm{r}}, l_{\eta \bar{s}}^{0}, u\right\rangle$. The $u$-independent label $l_{\eta s}$ in them is a short notation for the set of quantum numbers,

$$
l_{\eta s}=S_{\eta}, S_{s}, n_{\eta}, n_{s} \text { where } n_{\alpha}=S_{\alpha}+S_{\alpha}^{z}=0,1, \ldots, 2 S_{\alpha} \text { and } \alpha=\eta, s .
$$

Furthermore, the label $l_{\mathrm{r}}$ refers to the set of all remaining $u$-independent quantum numbers needed to uniquely specify an energy eigenstate $\left|l_{r}, l_{\eta s}, u\right\rangle$.

As for the spin-1/2XXX chain, the sets of $\left(2 S_{\eta}+1\right) \times\left(2 S_{s}+1\right)-1$ finite- $u$ energy eigenstates that are generated from each LWS are called here non-LWSs. For the present model this applies to energy and momentum eigenstates that are LWSs of only one of such algebras. Indeed, such states are not LWSs as defined above. For a LWS one then has that $n_{\eta}=n_{s}=0$ in Eq. (109). Hence $l_{\eta s}^{0}$ stands for $S_{\eta}, S_{s}, 0,0$. The non-LWSs $\left|l_{\mathrm{r}}, l_{\eta s}, u\right\rangle$ can be generated from the corresponding LWSs $\left|l_{\mathrm{r}}, l_{\eta s}^{0}, u\right\rangle$ 
as [243-245],

$$
\begin{aligned}
\left|l_{\mathrm{r}}, l_{\eta s}, u\right\rangle & =\prod_{\alpha=\eta, s}\left(\frac{1}{\sqrt{\mathcal{C}_{\alpha}}}\left(\hat{S}_{\alpha}^{+}\right)^{n_{\alpha}}\right)\left|l_{\mathrm{r}}, l_{\eta s}^{0}, u\right\rangle \text { where } \mathcal{C}_{\alpha}=\left(n_{\alpha} !\right) \prod_{j=1}^{n_{\alpha}}\left(2 S_{\alpha}+1-j\right), \\
n_{\alpha} & =1, \ldots, 2 S_{\alpha} \text { where } \alpha=\eta, s, \\
\hat{S}_{\eta}^{+} & =\left(\hat{S}_{\eta}^{-}\right)^{\dagger}=\sum_{j=1}^{L}(-1)^{j} c_{j, \downarrow}^{\dagger} c_{j, \uparrow}^{\dagger} \text { and } \hat{S}_{s}^{+}=\left(\hat{S}_{s}^{-}\right)^{\dagger}=\sum_{j=1}^{L} c_{j, \downarrow}^{\dagger} c_{j, \uparrow} .
\end{aligned}
$$

The model in its full Hilbert space can be described either directly within the BA solution [118,253] or by application onto the LWSs of the $\eta$-spin and spin $S U(2)$ symmetry algebras off-diagonal generators [243], as given in Eq. (110).

There are infinite unitary transformations such that,

$$
\hat{H} \rightarrow \hat{V}^{\dagger} \hat{H} \hat{V}=e^{-\hat{S}} \hat{H} e^{\hat{S}} \text { where } \hat{V}=e^{\hat{S}} .
$$

Within the physical problem studied in Refs. [254-259], the unitary operators $\hat{V}=e^{\hat{S}}$ transform the Hamiltonian $\hat{H}$, Eq. (105), into a rotated Hamiltonian $\hat{V}^{\dagger} \hat{H} \hat{V}$ other than $\hat{H}$. For it electron double and single occupancy are good quantum numbers for finite $u$ values. For large and intermediate $u$ values, the operator $\hat{S}$ can be expanded as $\hat{S}=-\sum_{i=0}^{\infty}\left(\frac{t}{U}\right)^{i} \hat{S}^{(i)}$. However, it is well-defined for the whole $u>0$ range. The infinite possible choices of operators $\hat{S}$ is an issue discussed in Ref. [254]. (The $\hat{S}$ expansion $\hat{S}=-\sum_{i=0}^{\infty}\left(\frac{t}{U}\right)^{i} \hat{S}^{(i)}$ is minus that in Eq. (57) of that reference.)

Nonetheless, exactly the same mathematical transformation, Eq. (111), can refer to the different physical problem discussed in this review. For the latter problem, $\hat{V}^{\dagger} \hat{H} \hat{V}$ is for $u>0$ the 1D Hubbard model written in the rotated electron representation. The creation and annihilation operators $\hat{V}^{\dagger} c_{j, \sigma}^{\dagger} \hat{V}$ and $\hat{V}^{\dagger} c_{j, \sigma} \hat{V}$ refer thus to rotated electrons rather than to electrons. Rotated-electron single and double occupancies are then good quantum numbers for the finite- $u$ 1D Hubbard model. In the $u \rightarrow \infty$ limit rotated-electron single and double occupancies become electron single and double occupancies, respectively. The global $c$-lattice $U(1)$ symmetry algebra generator beyond $S O(4)$ symmetry is the operator that counts the number of rotated-electron singly occupied sites for $u>0$. (It can also be chosen to be the operator that counts the number of rotated-electron unoccupied plus doubly occupied sites for $u>0$.)

The kinetic-energy operator $\hat{T}$, Eq. (106), can be written as $\hat{T}=\hat{T}_{0}+\hat{T}_{+1}+\hat{T}_{-1}$. The operator $\hat{T}_{0}$ conserves the number of rotated-electron doubly occupied sites. The operators $\hat{T}_{+1}$ and $\hat{T}_{-1}$ enhance and lessen it by one, respectively. The infinite electron-rotated-electron unitary transformations share an important property. It is that although the $u$ dependent expression of the operator $\hat{S}$ in the unitary operator $\hat{V}=e^{\hat{S}}$, Eq. (111), is different for each such a transformation, for $u>0$ it always involves only the three kinetic operators $\hat{T}_{0}, \hat{T}_{+1}$, and $\hat{T}_{-1}$. Another property common to all electron-rotated-electron unitary transformations is that to leading order in $t / U$, the operator $\hat{S}$, Eq. (111), has the universal form, $\hat{S}=-\frac{t}{U}\left(\hat{T}_{+1}-\hat{T}_{-1}\right)$. From a straightforward yet cumbersome algebra, one then finds that the momentum operator $\hat{P}$ and the six generators of the $\eta$-spin and spin $S U(2)$ symmetry algebras commute with the three kinetic operators $\hat{T}_{0}, \hat{T}_{+1}$, and $\hat{T}_{-1}$. This ensures that such operators commute with the electron-rotated-electron unitary operators $\hat{V}=e^{\hat{S}}$ and corresponding operators $\hat{S}$.

4.3. The Hubbard model BA uniquely defined rotated electrons and corresponding $c$ pseudoparticle, rotated spin, and rotated $\eta$-spin operators

The pseudoparticle representation and related pseudofermion representation of the 1D Hubbard model refer to a specific choice of the electron-rotated-electron unitary operator $\hat{V}$. The corresponding rotated-electron creation and annihilation operators and the number of rotated electrons at lattice site $j$ with spin projection $\sigma$ operator read,

$$
\tilde{c}_{j, \sigma}^{\dagger}=\hat{V}^{\dagger} c_{j, \sigma}^{\dagger} \hat{V}, \quad \tilde{c}_{j, \sigma}=\hat{V}^{\dagger} c_{j, \sigma} \hat{V} \text { and } \tilde{n}_{j, \sigma}=\tilde{c}_{j, \sigma}^{\dagger} \tilde{c}_{j, \sigma},
$$

respectively. Such rotated electrons are generated from the electrons by a unitary transformation defined and performed by the BA. The corresponding electron-rotated-electron unitary operator $\hat{V}$ in Eq. (112) is uniquely defined in Ref. [64] by its matrix elements between the model $4^{L}$ energy and momentum eigenstates.

In the $u \rightarrow \infty$ limit (and thus $u^{-1} \rightarrow 0$ limit) all spin configurations and all $\eta$-spin configurations with the same number of doubly occupied sites are degenerated. Hence there are in that limit infinite choices of complete sets of $4^{L}$ energy and momentum eigenstates for which electron single and double occupancies are good quantum numbers. The unitary transformation uniquely defined in Ref. [64] refers to a specific set of $4^{L}$ energy and momentum eigenstates. It is that obtained from the set of $4^{L}$ finite- $u$ energy eigenstates $\left|l_{\mathrm{r}}, l_{\eta s}, u\right\rangle$, Eq. (110), upon turning off adiabatically $u^{-1}$. The finite- $u$ energy eigenstates $\left|l_{\mathrm{r}}, l_{\eta s}, u\right\rangle=\hat{V}^{\dagger}\left|l_{\mathrm{r}}, l_{\eta s}, \infty\right\rangle$ generated from each such $u \rightarrow \infty$ energy eigenstates $\left|l_{\mathrm{r}}, l_{\eta s}, \infty\right\rangle$ have for $u>0$ exactly the same values for all $u$-independent quantum numbers. This includes the quantum numbers $l_{\eta s}$ given in Eq. (109) and all remaining $u$-independent quantum numbers $l_{\mathrm{r}}$ (provided below in Eq. (137)) needed to uniquely specify an energy eigenstate $\left|l_{\mathrm{r}}, l_{\eta s}, u\right\rangle$. A $V(u)$-set of states is our designation for such continuum set of $u>0$ energy eigenstates.

Important physical information can be reached from analysis of the relation between (i) the BA quantum numbers and (ii) the rotated-electron occupancy configurations, respectively, that generate the finite- $u$ exact energy eigenstates 
$\left|l_{\mathrm{r}}, l_{\eta s}, u\right\rangle=\hat{V}^{\dagger}\left|l_{\mathrm{r}}, l_{\eta s}, \infty\right\rangle$ of any $V(u)$-set. The rotated-electron spatial occupancy configurations that generate from the electron (and rotated-electron) vacuum the finite- $u$ energy eigenstates $\left|l_{\mathrm{r}}, l_{\eta s}, u\right\rangle=\hat{V}^{\dagger}\left|l_{\mathrm{r}}, l_{\eta s}, \infty\right\rangle$ of any $V(u)$-set of states are exactly the same as the electron spatial occupancy configurations that generate from it the corresponding $u \rightarrow \infty$ energy and momentum eigenstate $\left|l_{\mathrm{r}}, l_{\eta s}, \infty\right\rangle$. Hence for $u>0$ the number $N_{s, \pm 1 / 2}^{R}$ of spin-projection $\pm 1 / 2$ rotated-electron singly occupied sites, $N_{\eta,+1 / 2}^{R}$ of rotated-electron unoccupied sites, and $N_{\eta,-1 / 2}^{R}$ of rotated-electron doubly occupied sites are conserved.

Such numbers obey the sum rules $N_{s,+1 / 2}^{R}+N_{\eta,-1 / 2}^{R}=N_{e \uparrow}, N_{s,-1 / 2}^{R}+N_{\eta,-1 / 2}^{R}=N_{e \downarrow}, N_{s}^{R}+2 N_{\eta,-1 / 2}^{R}=N_{e}$, and $N_{s}^{R}+N_{\eta}^{R}=L$. The rotated-electron numbers equal those of the electrons. Therefore, here $N_{e \uparrow}$ and $N_{e \downarrow}$ denotes both the number of electrons and rotated electrons of spin projection $+1 / 2$ and $-1 / 2$, respectively. However, for finite $u$ values the numbers $N_{s}^{R}=N_{s,+1 / 2}^{R}+N_{s,-1 / 2}^{R}$ of rotated-electron singly occupied sites and $N_{\eta}^{R}=N_{\eta,+1 / 2}^{R}+N_{\eta,-1 / 2}^{R}$ of rotated-electron doubly occupied plus unoccupied sites are only conserved for rotated electrons.

For any operator $\hat{O}$ there is a corresponding operator $\tilde{O}=\hat{V}^{\dagger} \hat{O} \hat{V}$ whose expression in terms of rotated-electron creation and annihilation operators is the same as that of $\hat{O}$ in terms of electron creation and annihilation operators, respectively. The $l=z, \pm$ local rotated spins operators $(\alpha=s)$ and local rotated $\eta$-spin operators $(\alpha=\eta)$,

$$
\begin{aligned}
& \tilde{S}_{j, \alpha}^{l}=\hat{V}^{\dagger} \hat{S}_{j, \alpha}^{l} \hat{V} \text { where } l=z, \pm \text { and } \alpha=\eta, s, \\
& \tilde{S}_{j, \alpha}^{ \pm}=\tilde{S}_{j, \alpha}^{x} \pm i \tilde{S}_{j, \alpha}^{y} \text { where } \alpha=\eta, s,
\end{aligned}
$$

play a major role in the 1D Hubbard model pseudoparticle representation revisited below in Sections 5 and 6 .

Here $\hat{S}_{j, s}^{l}$ and $\hat{S}_{j, \eta}^{l}$ are the usual unrotated local spin $\eta$-spin operators, respectively. The rotated local operators $\tilde{S}_{j, \alpha}^{l}$, Eq. (113), have in terms of creation and annihilation rotated-electron operators, Eq. (112), exactly the same expressions as the corresponding unrotated local operators $\hat{S}_{j, \alpha}^{l}$ in terms of creation and annihilation electron operators. Specifically, the spin operators $\tilde{S}_{j, s}^{l}$, which act onto sites singly occupied by rotated electrons, read $\tilde{S}_{j, s}^{-}=\left(\tilde{S}_{j, s}^{+}\right)^{\dagger}=\tilde{c}_{j, \uparrow}^{\dagger} \tilde{c}_{j, \downarrow}$ and $\tilde{S}_{j, s}^{z}=\left(\tilde{n}_{j, \downarrow}-1 / 2\right)$. Similarly, the $\eta$-spin operators $\tilde{S}_{j, \eta}^{l}$, which act onto sites unoccupied by rotated electrons and sites doubly occupied by rotated electrons, are given by $\tilde{S}_{j, \eta}^{-}=\left(\tilde{S}_{j, \eta}^{+}\right)^{\dagger}=(-1)^{j} \tilde{c}_{j, \uparrow} \tilde{c}_{j, \downarrow}$ and $\tilde{S}_{j, \eta}^{z}=\left(\tilde{n}_{j, \downarrow}-1 / 2\right)$.

For $u>0$ a non-perturbative three degrees of freedom spin- $\eta$-spin- $c$-lattice separation occurs at all energy scales [64]. It naturally emerges from the independent state representations of the two $S U(2)$ symmetries and $c$-lattice $U(1)$ symmetry, respectively, in the model global symmetry. The $c$-lattice- $\eta$-spin degrees of freedom separation may be considered as a separation of the charge degrees of freedom. At zero temperature and energy scales lower than $2|\mu|$ relative to the ground state, one has that $N_{\eta,-1 / 2}^{R}=0$ (and $N_{\eta,+1 / 2}^{R}=0$ ) for $n_{e} \in\left[0,1\left[\right.\right.$ (and $\left.\left.n_{e} \in\right] 1,2\right]$ ). Hence for such energy ranges the $\eta$-spin degrees of freedom remain hidden. The three degrees of freedom non-perturbative $c$-lattice- $\eta$-spin-spin separation is then seen as the usual two degrees of freedom charge-spin separation.

Under the three general degrees of freedom separation of the rotated-electron occupancy configurations, their operators, Eq. (112), are of the form,

$$
\begin{aligned}
& \tilde{c}_{j, \uparrow}^{\dagger}=\left(\frac{1}{2}-\tilde{S}_{j, s}^{z}-\tilde{S}_{j, \eta}^{z}\right) f_{j, c}^{\dagger}+(-1)^{j}\left(\frac{1}{2}+\tilde{S}_{j, s}^{z}+\tilde{S}_{j, \eta}^{z}\right) f_{j, c} \text { and } \tilde{c}_{j, \uparrow}=\left(\tilde{c}_{j, \uparrow}^{\dagger}\right)^{\dagger}, \\
& \tilde{c}_{j, \downarrow}^{\dagger}=\left(\tilde{S}_{j, s}^{+}+\tilde{S}_{j, \eta}^{+}\right)\left(f_{j, c}^{\dagger}+(-1)^{j} f_{j, c}\right) \text { and } \tilde{c}_{j, \downarrow}=\left(\tilde{c}_{j, \downarrow}^{\dagger}\right)^{\dagger} .
\end{aligned}
$$

Here the operators $f_{j, c}^{\dagger}$ and $f_{j, c}$ defined below create and annihilate one $c$ pseudoparticle at the $c$ effective lattice site $j=1, \ldots, L$. That lattice is identical to the electron and rotated-electron original lattice. Their (i) occupied and (ii) unoccupied sites are those (i) singly occupied and (ii) unoccupied and doubly occupied by the rotated electrons, respectively. Hence the $c$ pseudoparticle local density operator $\tilde{n}_{j, c} \equiv f_{j, c}^{\dagger} f_{j, c}$ and the corresponding operator $\left(1-\tilde{n}_{j, c}\right)$ are the natural projectors onto the subset of $N_{R}^{s}=N_{c}$ original-lattice sites singly occupied by rotated electrons and onto the subset of $N_{R}^{\eta}=N_{c}^{h}=L-N_{c}$ original-lattice sites unoccupied and doubly occupied by rotated electrons, respectively. It then follows that the local operators $\tilde{S}_{j, \alpha}^{l}$, Eq. (113), can be written as,

$$
\tilde{S}_{j, s}^{l}=\tilde{n}_{j, c} \tilde{q}_{j}^{l} \text { and } \tilde{S}_{j, \eta}^{l}=\left(1-\tilde{n}_{j, c}\right) \tilde{q}_{j}^{l} \text { where } l=z, \pm,
$$

respectively. Here the $l=z, \pm$ local $\eta s$ quasi-spin operators,

$$
\tilde{q}_{j}^{l}=\tilde{S}_{j, s}^{l}+\tilde{S}_{j, \eta}^{l} \text { where } l= \pm, z,
$$

such that $\tilde{q}_{j}^{ \pm}=\tilde{q}_{j}^{x} \pm i \tilde{q}_{j}^{y}$, have the following expression in terms of rotated-electron creation and annihilation operators,

$$
\tilde{q}_{j}^{-}=\left(\tilde{q}_{j}^{+}\right)^{\dagger}=\left(\tilde{c}_{j, \uparrow}^{\dagger}+(-1)^{j} \tilde{c}_{j, \uparrow}\right) \tilde{c}_{j, \downarrow} \text { and } \tilde{q}_{j}^{z}=\left(\tilde{n}_{j, \downarrow}-1 / 2\right) \text {. }
$$

The local $c$ pseudoparticle operators $f_{j, c}^{\dagger}$ and $f_{j, c}$ in Eq. (114) are uniquely defined for $u>0$ in terms of rotated-electron creation and annihilation operators, Eq. (112). This is achieved by combining the inversion of the relations, Eq. (114), with 
the expressions of the local operators $\tilde{S}_{j, s}^{l}$ and $\tilde{S}_{j, \eta}^{l}$ provided in Eqs. (115)-(117). This gives,

$$
f_{j, c}^{\dagger}=\left(f_{j, c}\right)^{\dagger}=\tilde{c}_{j, \uparrow}^{\dagger}\left(1-\tilde{n}_{j, \downarrow}\right)+(-1)^{j} \tilde{c}_{j, \uparrow} \tilde{n}_{j, \downarrow} \text { and } \tilde{n}_{j, c}=f_{j, c}^{\dagger} f_{j, c} \text { for } j=1, \ldots, L .
$$

The operator $\tilde{n}_{j, \sigma}$ in this equation is the $\sigma$ rotated-electron local density operator given in Eq. (112). (In the $u \rightarrow \infty$ limit, the $\eta s$ quasi-spins associated with the operators, Eq. (117), and the $c$ pseudoparticle holes associated with operators $f_{j, c}$, Eq. (118), become the quasispins and quasicharges, respectively, of Ref. [260].)

On the one hand, the rotated spins $1 / 2$ of projection $\pm 1 / 2$ are the spin- $1 / 2$ fractionalized particles associated with the $l=z, \pm$ spin operators $\tilde{S}_{j, s}^{l}$ in Eq. (115). They refer to the $L_{s, \pm 1 / 2}=N_{s, \pm 1 / 2}^{R}$ spins $1 / 2$ of the rotated electrons with such a spin projection that singly occupy sites. On the other hand, the rotated $\eta$-spins $1 / 2$ of projection $\pm 1 / 2$ are the $\eta$-spin- $1 / 2$ fractionalized particles associated with the $l=z, \pm \eta$-spin operators $\tilde{S}_{j, \eta}^{l}$ in Eq. (115). They refer to the $\eta$-spin degrees of freedom of the $L_{\eta, \pm 1 / 2}=N_{\eta, \pm 1 / 2}^{R}$ sites unoccupied $(+1 / 2)$ and doubly occupied $(-1 / 2)$ by rotated electrons.

The charge and spin of the electrons remain invariant under the electron-rotated-electron unitary transformation. Indeed, it only changes their spatial original lattice occupancy distributions. Therefore, the rotated spins $1 / 2$ and rotated $\eta$-spins $1 / 2$ are physical particles with a well-defined relation to the rotated electrons and corresponding electrons. There is a rotated spin $1 / 2$ and rotated $\eta$-spin $1 / 2$ quantum problem for the $1 \mathrm{D}$ Hubbard model in each fixed- $N_{c}$ subspace where $N_{c}=N_{s}^{R} \in[0, L]$. The reason is that only in such subspaces are the numbers $L_{s}=N_{s}^{R}=N_{c}$ of rotated spins $1 / 2$ and $L_{\eta}=N_{\eta}^{R}=L-N_{c}$ of rotated $\eta$-spins $1 / 2$, respectively, fixed. The rotated spin $1 / 2$ and rotated $\eta$-spin $1 / 2$ representation is well defined in such subspaces.

For simplicity, in the remaining of this review the rotated spins $1 / 2$ and rotated $\eta$-spins $1 / 2$ are called spins $1 / 2$ and $\eta$-spins $1 / 2$, respectively. The spins $1 / 2$ are though only those carried by the rotated electrons that singly occupy original lattice sites. Those within the rotated electrons doubly occupied original lattice sites rather refer to the $\eta$-spin $S U(2)$ symmetry algebra. Indeed, such doubly occupied original lattice sites $\eta$-spin degrees of freedom correspond to the $\eta$-spins of $\eta$-spin projection $-1 / 2$. (The unoccupied original lattice sites $\eta$-spin degrees of freedom refer to the $\eta$-spins of $\eta$-spin projection $+1 / 2$.)

Within the above general separation, the (i) global $c$-lattice $U(1)$ symmetry, (ii) global $\eta$-spin $S U(2)$ symmetry, and (iii) global spin $S U(2)$ symmetry state representations are, in each subspace with a fixed number $N_{s}^{R}$ of rotated-electron singly occupied sites, generated by three sets of independent occupancy configurations. Those involve: (i) The $N_{c}=N_{s}^{R}$ c pseudoparticles without internal degrees of freedom and corresponding $N_{c}^{h}=N_{\eta}^{R} c$ pseudoparticle holes; (ii) The $L_{s, \pm 1 / 2}=N_{s, \pm 1 / 2}^{R}$ spins $1 / 2$ of projection $\pm 1 / 2$; (iii) The $L_{\eta, \pm 1 / 2}=N_{\eta,+1 / 2}^{R} \eta$-spins $1 / 2$ of projection $\pm 1 / 2$. It then follows that their numbers are such that,

$$
\begin{aligned}
& L_{s}=L_{s,+1 / 2}+L_{s,-1 / 2}=N_{c}, \\
& L_{\eta}=L_{\eta,+1 / 2}+L_{\eta,-1 / 2}=L-N_{c}=N_{c}^{h}, \\
& L_{s,+1 / 2}-L_{s,-1 / 2}=-2 S_{s}^{z}=N_{e \uparrow}-N_{e \downarrow}, \\
& L_{\eta,+1 / 2}-L_{\eta,-1 / 2}=-2 S_{\eta}^{z}=L-N_{e} .
\end{aligned}
$$

Here $L_{s}$ denotes the number of spins and $L_{\eta}$ that of $\eta$-spins. Those equal the numbers $N_{c}$ of $c$ pseudoparticles and $N_{c}^{h}=L-N_{c}$ of $c$ pseudoparticle holes, respectively.

The numbers $N_{c}$ of $c$ pseudoparticles, $L_{\eta, \pm 1 / 2}$ of $\eta$-spins of projection $\pm 1 / 2$, and $L_{s, \pm 1 / 2}$ of spins of projection $\pm 1 / 2$ are fully controlled by those of rotated electrons as follows,

$$
\begin{aligned}
& N_{c}=N_{R}^{s}, \quad N_{c}^{h}=N_{R}^{\eta} \text { and } N_{c}+N_{c}^{h}=N_{R}^{s}+N_{R}^{\eta}=L, \\
& L_{\alpha, \pm 1 / 2}=N_{R, \pm 1 / 2}^{\alpha} \text { and } L_{\alpha}=L_{\alpha,+1 / 2}+L_{\alpha,-1 / 2}=N_{R}^{\alpha} \text { where } \alpha=\eta, s .
\end{aligned}
$$

This is consistent with such fractionalized particles stemming from the rotated-electron occupancy configurations degrees of freedom separation.

The global three degrees of freedom rotated-electron separation leads locally in what the onsite rotated-electron occupancies is concerned to two degrees of freedom separation. On the one hand, the degrees of freedom of each rotatedelectron occupied site decouple into one spin-less $c$ pseudoparticle without internal degrees of freedom and one spin $1 / 2$ that carries its spin. On the other hand, the degrees of freedom of each rotated-electron unoccupied and doubly occupied site decouple into one $c$ pseudoparticle hole and one $\eta$-spin $1 / 2$ of projection $+1 / 2$ and $-1 / 2$, respectively. Hence the local two degrees of freedom separation corresponds to those of the $c$-lattice $U(1)$ symmetry and one of the two global $S U(2)$ symmetries, respectively.

The unitarity of the electron-rotated-electron transformation implies that the rotated-electron operators $\tilde{c}_{j, \sigma}^{\dagger}$ and $\tilde{c}_{j, \sigma}$, Eqs. (112) and (114), have the same anti-commutation relations as the corresponding electron operators $c_{j, \sigma}^{\dagger}$ and $c_{j, \sigma}$, respectively. Straightforward manipulations based on Eqs. (113)-(118) then lead to the following algebra for the local $c$ pseudoparticle creation and annihilation operators,

$$
\left\{f_{j, c}^{\dagger}, f_{j^{\prime}, c}\right\}=\delta_{j, j^{\prime}} \text { and }\left\{f_{j, c}^{\dagger}, f_{j^{\prime}, c}^{\dagger}\right\}=\left\{f_{j, c}, f_{j^{\prime}, c}\right\}=0 .
$$


Furthermore, the local $c$ pseudoparticle operators and the local rotated quasi-spin operators $\tilde{q}_{j}^{l}$, Eq. (117), commute with each other. From the use of Eqs. (113)-(117) one confirms that the $S U(2)$ algebra obeyed by the local quasi-spin operators $\tilde{q}_{j}^{l}$ is the usual one,

$$
\left[\tilde{q}_{j}^{+}, \tilde{q}_{j^{\prime}}^{-}\right]=\delta_{j, j^{\prime}} 2 \tilde{q}_{j}^{z} \text { and }\left[\tilde{q}_{j}^{ \pm}, \tilde{q}_{j^{\prime}}^{z}\right]=\mp \delta_{j, j^{\prime}} \tilde{q}_{j}^{ \pm} \text {. }
$$

The same applies to the $S U(2)$ algebras of the corresponding (rotated) $\eta$-spin and spin operators $\tilde{s}_{j, \eta}^{l}$ and $\tilde{s}_{j, s}^{l}$, respectively. Moreover, $\left[\tilde{q}_{j}^{l}, \tilde{q}_{j^{\prime}}^{l}\right]=0$ and $\left[\tilde{s}_{j, \alpha}^{l}, \tilde{s}_{j^{\prime}, \alpha^{\prime}}^{l}\right]=0$. The $c$ pseudoparticle and $\eta s$ quasi-spin operator algebras refer to the whole Hilbert space. In contrast, those of the (rotated) $\eta$-spin and spin operators correspond to fixed- $N_{c}$ subspaces.

The degrees of freedom separation, Eq. (114), is such that the $c$ pseudoparticle operators, Eq. (118), (rotated) spin $1 / 2$ and $\eta$-spin 1/2 operators, Eq. (115), and the related $\eta s$ quasi-spin operators, Eqs. (116) and (117), emerge from the rotated-electron operators by an exact local transformation that does not introduce constraints. The expressions of the c pseudoparticle, spin $1 / 2$, and $\eta$-spin $1 / 2$ operators in terms of rotated-electron creation and annihilation operators are valid for $u>0$. The latter operators are related to the original electron creation and annihilation operators through the transformation, Eq. (112). The unitary operator in that transformation is uniquely defined in Ref. [64] by its $4^{L}$ matrix elements. Combination of all such equations thus uniquely defines for $u>0$ the $c$ pseudoparticle, spin $1 / 2$, and $\eta$-spin $1 / 2$ operators in terms of electron creation and annihilation operators.

In the case of the spin- $1 / 2 X X X$ chain, the number $L$ of sites singly occupied by spins $1 / 2$ is a good quantum number. The emergence within the $u>0$ 1D Hubbard model rotated-electron representation of $L_{s}=N_{R}^{s}$ spins $1 / 2$ that singly occupy $L_{s}=N_{c}$ original-lattice sites renders the problem much similar to that of such a chain. Also the $L_{\eta}=N_{R}^{\eta} \eta$-spins $1 / 2$ singly occupy $L_{\eta}=N_{c}^{h}=L-N_{c}$ original-lattice sites. As justified in Appendix F, the present rotated-electron representation spin$1 / 2$ and $\eta$-spin-1/2 occupancy configurations that generate the two $S U(2)$ symmetries degrees of freedom of the energy and momentum eigenstates of the 1D Hubbard model for $u>0$ are actually exactly the same as those that generate the energy and momentum eigenstates of a spin-1/2 and $\eta$-spin-1/2 XXX chain with $L_{s}=N_{c}$ and $L_{\eta}=N_{c}^{h}=L-N_{c}$ sites, respectively. The relation to the 1D Lieb-Liniger Bose gas is brought about by the independent occupancy configurations of the $c$ pseudoparticles, which have no internal structure. And as the pseudoparticles of that gas, they are associated with a $U(1)$ symmetry. Indeed, they generate the $c$-lattice $U(1)$ symmetry degrees of freedom of the energy and momentum eigenstates of the 1D Hubbard model for $u>0$.

As mentioned above, the $c$ pseudoparticles live on a $c$ effective lattice similar to the original lattice. In contrast, the (i) spins $1 / 2$ and (ii) $\eta$-spins $1 / 2$ only "see" the sites (i) singly occupied and (ii) unoccupied and doubly occupied, respectively, by rotated electrons. Hence for the model in fixed- $N_{c}$ subspaces one can define within the TL a squeezed spin effective lattice with $L_{s}=N_{s}^{R}=N_{c}$ sites on which the spins $1 / 2$ live. One can define as well a corresponding squeezed $\eta$-spin effective lattice with $L_{\eta}=N_{\eta}^{R}=L-N_{c}$ sites for the $\eta$-spins $1 / 2$. The numbers of sites of such squeezed $\eta$-spin and spin effective lattices are thus given by,

$$
L_{\eta}=N_{c}^{h}=L-N_{c} \text { and } L_{s}=N_{c},
$$

respectively. The squeezed $\eta$-spin and spin effective lattices remain the same for the $\left(2 S_{\eta}+1\right) \times\left(2 S_{s}+1\right)-1$ nonLWSs $\left|l_{\mathrm{r}}, l_{\eta s}, u\right\rangle$ generated from the LWSs, Eq. (110). Their configurations in such lattices are those of the non-LWSs of the corresponding $\eta$-spin-1/2 and spin- $1 / 2 X X X$ chains with $L_{\eta}$ and $L_{S}$ sites, respectively.

Squeezed spaces are actually well known from studies of the 1D Hubbard model in the $u \rightarrow \infty$ limit [119,150,156$158,261]$. Such studies have used the $u \rightarrow \infty$ energy and momentum eigenstates $\left|l_{\mathrm{r}}, l_{\eta s}, \infty\right\rangle$ associated with the BA solution considered in this paper. In Appendix F some of the $u \rightarrow \infty$ properties in terms of electron occupancy configurations that generate the states $\left|l_{\mathrm{r}}, l_{\eta s}, \infty\right\rangle$ are extended to the $u>0$ range in terms of rotated electrons.

The $c$ effective lattice, $\eta$-spin effective lattice, and spin effective lattice occupancy configurations are independent. The role of the representations of the $c$-lattice $U(1)$ symmetry generated by the $c$ pseudoparticle occupancy configurations is indeed to store the information on the positions in the original lattice of the $N_{R}^{s}=N_{c}$ sites singly occupied by rotated electrons ( $c$ pseudoparticles) relative to the $N_{R}^{\eta}=N_{c}^{h}$ sites doubly occupied and unoccupied by rotated electrons ( $c$ band holes). This ensures that the spin effective lattice occupancies of the $L_{s}=N_{R}^{s}=N_{c}$ spins $1 / 2$ and $\eta$-spin effective lattice occupancies of the $L_{\eta}=N_{R}^{\eta}=N_{c}^{c} \eta$-spins $1 / 2$ associated with the spin and $\eta$-spin $S U(2)$ symmetries, respectively, are independent.

It follows from such an independence that within the TL the spin $(\alpha=s)$ and $\eta$-spin $(\alpha=\eta)$ effective lattice sites locations can be associated with their fixed- $N_{c}$ subspace average locations at $x=a_{\alpha} j$ where $j=1, \ldots, L_{\alpha}$. The spin effective lattice spacing $a_{s}$ and $\eta$-spin effective lattice spacing $a_{\eta}$ thus correspond to the average spacing between the $c$ effective lattice occupied sites and between such a lattice unoccupied sites, respectively, in the corresponding $N_{c}$-fixed subspace. This gives,

$$
a_{\alpha}=\frac{L}{L_{\alpha}}=\frac{L}{L_{\alpha}} a \text { where } \alpha=\eta, s .
$$

This spacing ensures that the $\eta$-spin $(\alpha=\eta)$ and $\operatorname{spin}(\alpha=s)$ effective lattices have exactly the same length as the original lattice. The effective lattice spacings, Eq. (124), are in general larger than that of the original lattice. The exception refers to subspaces for which $n_{c} \rightarrow 1$ and $n_{c}^{h} \rightarrow 1$. For them these sites numbers read $L_{s}=L ; L_{\eta}=0$ and $L_{s}=0 ; L_{\eta}=L$, respectively. Hence in these two density limits the spin and $\eta$-spin effective lattice becomes the original lattice and the $\eta$-spin and spin effective lattice does not exist, respectively. 


\subsection{Unpaired and paired spins and $\eta$-spins}

The energy eigenstates that span a fixed- $N_{c}$ subspace are a superposition of $c$ effective lattice, spin effective lattice, and $\eta$-spin effective lattice occupancy configurations. As discussed in Appendix C, the two degrees of freedom separation of the rotated-electron occupancies of each of the $L$ original lattice sites is behind the two sum rules given in Eq. (C.3) of that Appendix.

For the energy eigenstates of spin $(\alpha=s)$ or $\eta$-spin $(\alpha=\eta) S_{\alpha} \leq L_{\alpha} / 2$ in a fixed- $N_{c}$ subspace, the corresponding spin or $\eta$-spin effective lattice occupancy configurations have a number $2 S_{\alpha}$ of sites occupied by a set of $M_{\alpha}=2 S_{\alpha}$ spins $1 / 2$ $(\alpha=s)$ or $\eta$-spins $1 / 2(\alpha=\eta)$ that participate in the (spin or $\eta$-spin) multiplet configuration. They have in addition a complementary set of even number $L_{\alpha}-2 S_{\alpha}$ spin or $\eta$-spin effective lattice sites. Those are singly occupied by $L_{\alpha}-2 S_{\alpha}$ spins $1 / 2$ or $\eta$-spins $1 / 2$, respectively, whose configuration forms a tensor product of (spin or $\eta$-spin) singlet states. Such results are those expected from the direct relation to the $\eta$-spin-1/2 and spin-1/2XXX chains with $L_{\eta}$ and $L_{s}$ sites, respectively.

Such an analysis applies as well in terms of the spin and $\eta$-spin degrees of freedom of the original lattice sites rotatedelectron occupancy configurations. Indeed, the spin $1 / 2$ occupancy configuration order in the spin effective lattice is exactly the same as that of the spins of the rotated electrons that singly occupy sites in the original lattice. This is independent of the positions in it of the sites unoccupied and doubly occupied by rotated electrons. Similarly, the $\eta$-spin $1 / 2$ occupancy configuration order in the $\eta$-spin effective lattice is exactly the same as that of the rotated-electron doubly and unoccupied sites in the original lattice. Again, this is independent of the positions in it of the sites singly occupied by rotated electrons. Consistently, the spin-1/2 XXX chain distribution of the squeezed spin wave function $\phi_{S U(2)}^{s}\left(x^{\varsigma \downarrow}, \ldots\right.$ ) in Eq. (F.1) of Appendix F does not change if the chain of rotated-electron singly occupied sites is "diluted" by rotated-electron unoccupied and doubly occupied sites. The same applies to the $\eta$-spin-1/2XXX chain distribution of the squeezed $\eta$-spin wave function $\phi_{S U(2)}^{\eta}\left(x^{d}, \ldots\right)$ in that equation if the chain of rotated-electron unoccupied and doubly occupied sites is "diluted" by rotated-electron singly occupied sites.

All the energy and momentum eigenstates with the same $S_{\alpha}$ have the same $\operatorname{spin}(\alpha=s)$ or $\eta$-spin $(\alpha=\eta) \hat{\vec{S}}_{\alpha}^{2}$ eigenvalue. Therefore, the energy and momentum eigenstates are superpositions of the corresponding above two types of configuration terms. Each term in them is characterized by a different partition of $L_{\alpha}$ spins $1 / 2(\alpha=s)$ or $\eta$-spins $1 / 2(\alpha=\eta)$ into two types of configurations. $M_{\alpha}=2 S_{\alpha}$ such spins or $\eta$-spins, respectively, participate in a $2 S_{\alpha}+1$ (spin or $\eta$-spin) multiplet. The remaining even number $L_{\alpha}-2 S_{\alpha}$ of spins $1 / 2$ or $\eta$-spins $1 / 2$ participate in a product of (spin or $\eta$-spin) singlets. The latter are associated with a corresponding number,

$$
\Pi_{\alpha}=\frac{1}{2}\left(L_{\alpha}-2 S_{\alpha}\right) \text { where } \alpha=s, \eta,
$$

of $\operatorname{spin}(\alpha=s)$ or $\eta$-spin $(\alpha=\eta)$ singlet pairs. In the following they are often generally called $\alpha$-singlet pairs.

The unpaired spins and paired spins $(\alpha=s)$ and unpaired $\eta$-spins and paired $\eta$-spins $(\alpha=\eta)$ are the members of such two sets of $M_{\alpha}=2 S_{\alpha}$ and $2 \Pi_{\alpha}=L_{\alpha}-2 S_{\alpha}$, respectively, spins $1 / 2$ and $\eta$-spins $1 / 2$. For a spin and $\eta$-spin LWS, all unpaired spins $1 / 2$ and unpaired $\eta$-spins $1 / 2$, respectively, have projection $+1 / 2$.

The number of pairs $\Pi_{\alpha}$ is directly related to the spin $S U(2)$ symmetry $(\alpha=s)$ and $\eta$-spin $S U(2)$ symmetry $(\alpha=\eta)$ in the $[S O(4) \otimes U(1)] / Z_{2}=[S U(2) \otimes S U(2) \otimes U(1)] / Z_{2}^{2}$ global symmetry of the $u>0$ 1D Hubbard model Hamiltonian. Indeed, the expression, Eq. (C.4) of Appendix C, of the number $\mathcal{N}_{\text {singlet }}\left(S_{\alpha}, L_{\alpha}\right)$ of that model independent spin $(\alpha=s)$ and $\eta$-spin $(\alpha=\eta)$ $\alpha$-singlet state representations in a fixed- $N_{c}$ and fixed- $S_{\alpha}$ subspace is a function of only the number of pairs $\Pi_{\alpha}$ and of the number of spins $1 / 2(\alpha=s)$ and $\eta$-spins $1 / 2(\alpha=\eta) L_{\alpha}$.

For general $u>0$ LWSs and their non-LWSs one finds that the number $M_{s, \pm 1 / 2}$ of unpaired spins of projection $\pm 1 / 2$ and $M_{\eta, \pm 1 / 2}$ of unpaired $\eta$-spins of projection $\pm 1 / 2$ are good quantum numbers. They read,

$$
M_{\alpha, \pm 1 / 2}=\left(S_{\alpha} \mp S_{\alpha}^{z}\right) \text { and } M_{\alpha}=M_{\alpha,-1 / 2}+M_{\alpha,+1 / 2}=2 S_{\alpha} \text { where } \alpha=\eta, s .
$$

The set of an energy and momentum eigenstate $\Pi_{\eta} \eta$-spin-singlet pairs and $\Pi_{s}$ spin-singlet pairs contains an equal number of $\eta$-spins $1 / 2$ and spins $1 / 2$, respectively, of opposite projection. Hence the total number $L_{\alpha, \pm 1 / 2}$ of $\eta$-spins of projection $\pm 1 / 2(\alpha=\eta)$ and spins of projection $\pm 1 / 2(\alpha=s)$ is given by,

$$
L_{\alpha, \pm 1 / 2}=\Pi_{\alpha}+M_{\alpha, \pm 1 / 2}=\frac{1}{2}\left(L_{\alpha} \mp 2 S_{\alpha}^{z}\right) \text { where } \alpha=\eta, s .
$$

The $\eta$-spin and spin $S U(2)$ symmetry algebras diagonal generators $\hat{S}_{\eta}^{z}$ and $\hat{S}_{s}^{z}$, Eq. (107), and off-diagonal generators $\hat{S}_{\eta}^{+}$, $\hat{S}_{\eta}=\left(\hat{S}_{\eta}^{+}\right)^{\dagger}$ and $\hat{S}_{s}^{+}, \hat{S}_{s}=\left(\hat{S}_{s}^{+}\right)^{\dagger}$, Eq. (110), commute with the electron-rotated-electron unitary operator $\hat{V}$, Eq. (112). Hence such operators have the same expressions in terms of electron and rotated-electron operators. The $M_{\alpha}=2 S_{\alpha}$ unpaired spins $(\alpha=s)$ and unpaired $\eta$-spins $(\alpha=\eta)$ multiplet configurations are as given in Eq. (110) generated by application of the $\alpha=\eta, s$ operators $\hat{S}_{\alpha}^{+}$onto the LWSs. Therefore, the corresponding non-LWSs original lattice spatial occupancy configurations of the unpaired spins and unpaired $\eta$-spins generated from the LWSs are for the whole $u>0$ range exactly the same in terms of rotated electrons and electrons, respectively. Indeed, for $u>0$ such local configurations remain invariant under the electron-rotated-electron unitary transformation. Hence the unpaired spins $(\alpha=s)$ and unpaired $\eta$-spins $(\alpha=\eta)$ 
are for $u>0$ not rotated. They thus refer to electron unpaired physical spins $1 / 2$ and to the $\eta$-spin degrees of freedom of physical onsite spin-singlet electron pairs, respectively.

The $\alpha$-singlet configurations of the $2 \Pi_{\alpha}=L_{\alpha}-2 S_{\alpha}$ paired spins $1 / 2(\alpha=s)$ and paired $\eta$-spins $1 / 2(\alpha=\eta)$ left over are though also physical spins $1 / 2$ and physical $\eta$-spins $1 / 2$ in what their spin and $\eta$-spin degrees of freedom, respectively, is concerned. Only their original lattice spatial occupancies are changed under the electron-rotated-electron unitary transformation.

\section{The 1D Hubbard model $c$ and $\alpha n$ pseudoparticle representation}

The relation of the different types of the 1D Hubbard model pseudoparticles and band holes to its physical particles, the electrons, is much more involved than for the 1D Lieb-Liniger Bose gas and spin-1/2 XXX chain. Nonetheless, the relation of the latter model physical spins $1 / 2$ to its $n$-pseudoparticles and holes plays a valuable role in the study of the corresponding more complex problem of the 1D Hubbard model.

Here the functional representation of the 1D Hubbard model TBA solution is related to the three basic fractionalized particles that naturally arise from the rotated electrons degrees of freedom separation. The composite $\alpha n$ pseudoparticles emerge from such a relation. The charge and spin current carriers and the general $c$-band and $\alpha n$-bands hole representation is an issue also discussed in this section.

\subsection{The functional representation of the 1D Hubbard model TBA solution}

Some of the 1D Hubbard model TBA solution quantities and equations introduced in Ref. [73] needed for our analysis are provided here within a suitable distribution functional representation. The model TBA equations are within such a representation given by,

$$
\begin{aligned}
q_{j} & =k^{c}\left(q_{j}\right)+\frac{2}{L} \sum_{n=1}^{\infty} \sum_{j^{\prime}=1}^{L_{s n}} N_{s n}\left(q_{j^{\prime}}\right) \arctan \left(\frac{\sin k^{c}\left(q_{j}\right)-\Lambda^{s n}\left(q_{j^{\prime}}\right)}{n u}\right) \\
& +\frac{2}{L} \sum_{n=1}^{\infty} \sum_{j^{\prime}=1}^{L_{\eta n}} N_{\eta n}\left(q_{j^{\prime}}\right) \arctan \left(\frac{\sin k^{c}\left(q_{j}\right)-\Lambda^{\eta n}\left(q_{j^{\prime}}\right)}{n u}\right) \text { for } j=1, \ldots, L,
\end{aligned}
$$

and

$$
\begin{aligned}
q_{j}= & \delta_{\alpha, \eta} \sum_{\iota= \pm 1} \arcsin \left(\Lambda^{\alpha n}\left(q_{j}\right)-i \iota n u\right)+\frac{2(-1)^{\delta_{\alpha, \eta}}}{L} \sum_{j^{\prime}=1}^{L_{c}} N_{c}\left(q_{j^{\prime}}\right) \arctan \left(\frac{\Lambda^{\alpha n}\left(q_{j}\right)-\sin k^{c}\left(q_{j^{\prime}}\right)}{n u}\right) \\
- & \frac{1}{L} \sum_{n^{\prime}=1}^{\infty} \sum_{j^{\prime}=1}^{L_{\alpha n^{\prime}}} N_{\alpha n^{\prime}}\left(q_{j^{\prime}}\right) \Theta_{n n^{\prime}}\left(\frac{\Lambda^{\alpha n}\left(q_{j}\right)-\Lambda^{\alpha n^{\prime}}\left(q_{j^{\prime}}\right)}{u}\right) \text { for } j=1, \ldots, L_{\alpha n}, \\
& \text { where } \alpha=\eta, s \text { and } n=1, \ldots, \infty .
\end{aligned}
$$

The $\mathrm{BA} \beta=c, \alpha n$ branches numbers $L_{\beta}$ appearing in these equations read,

$$
\begin{aligned}
L_{c} & =N_{c}+N_{c}^{h}=N_{s}^{R}+N_{\eta}^{R}=L \text { where } N_{c}^{h}=L-N_{c}, \\
L_{\alpha n} & =N_{\alpha n}+N_{\alpha n}^{h} \text { where } N_{\alpha n}^{h}=2 S_{\alpha}+\sum_{n^{\prime}=n+1}^{\infty} 2\left(n^{\prime}-n\right) N_{\alpha n^{\prime}}, \\
N_{\beta} & =\sum_{j=1}^{L_{\beta}} N_{\beta}\left(q_{j}\right) \text { where } \beta=c, \eta n, s n \text { and } n=1, \ldots, \infty .
\end{aligned}
$$

The function $\Theta_{n n^{\prime}}(x)$ in Eqs. (128) and (129) is given in Eq. (B.1) of Appendix B and the $\beta$-branch discrete quantum numbers $q_{j}$ read,

$$
q_{j}=\frac{2 \pi}{L} I_{j}^{\beta} \text { for } j=1, \ldots, L_{\beta} \text { where } \beta=c, \eta n, \text { sn and } n=1, \ldots, \infty .
$$

Here $\left\{I_{j}^{\beta}\right\}$ are the $\beta$-branch $j=1, \ldots, L_{\beta}$ quantum numbers $\left\{q_{j}\right\}$ in units of $2 \pi / L$. Those are either integers or half-odd integers according to the following boundary conditions [73],

$$
\begin{aligned}
I_{j}^{\beta} & =0, \pm 1, \pm 2, \ldots \text { for } I_{\beta} \text { even }, \\
& = \pm 1 / 2, \pm 3 / 2, \pm 5 / 2, \ldots \text { for } I_{\beta} \text { odd },
\end{aligned}
$$


where,

$$
I_{c}=N^{S U(2)} \equiv \sum_{\alpha=\eta, s} \sum_{n=1}^{\infty} N_{\alpha n} \text { and } I_{\alpha n}=L_{\alpha n}-1 \text { for } \alpha=\eta, s \text { and } n=1, \ldots, \infty .
$$

The $\beta=c$, $\alpha$ n branch successive set of discrete values $q_{j}$, Eq. (131), have fixed separation, $q_{j+1}-q_{j}=2 \pi / L$. In addition, they have only occupancies zero and one. The $\beta$-branch distribution functions $N_{\beta}\left(q_{j}\right)$ in Eqs. (128) and (129) thus read $N_{\beta}\left(q_{j}\right)=1$ and $N_{\beta}\left(q_{j}\right)=0$ for occupied and unoccupied such discrete values, respectively. Each energy and momentum eigenstate is described by different occupancy configurations of the distributions $\left\{N_{\beta}\left(q_{j}\right)\right\}$ corresponding to all BA $\beta=c, \eta n$, sn branches where $n=1, \ldots, \infty$. The numbers $N_{\beta}$ and $N_{\beta}^{h}$ defined in Eq. (130) are thus those of occupied and unoccupied, respectively, $\beta$-branch discrete values $q_{j}$, Eq. (131).

Solution of the coupled TBA equations, Eqs. (128) and (129), provides the real momentum rapidity function $k^{c}\left(q_{j}\right)$ and the set of $n=1, \ldots, \infty$ real rapidity functions $\Lambda_{\eta n}\left(q_{j}\right)$ and $\Lambda_{s n}\left(q_{j}\right)$ of each energy and momentum eigenstate. Quantities such as the energy eigenvalues given in Eqs. (B.5)-(B.8) of Appendix B [69-71,73] and the charge and spin current operators expectation values depend on the $\beta$-branches discrete values $q_{j}$ through the dependence on them of the momentum rapidity function $k^{c}\left(q_{j}\right)$ and the $\alpha=\eta, s$ and $n=1, \ldots, \infty$ rapidity functions $\Lambda_{\alpha n}\left(q_{j}\right)$. The latter are the real part of TBA complex rapidities of general form,

$$
\Lambda^{\alpha n, l}\left(q_{j}\right)=\Lambda^{\alpha n}\left(q_{j}\right)+i(n+1-2 l) u \text { where } \alpha=\eta, s, \quad n=1, \ldots, \infty \text { and } l=1, \ldots, n .
$$

For $n=1$ this rapidity is real and otherwise its imaginary part is finite. A TBA $\alpha n$-string is a group of $l=1, \ldots, n$ rapidities, Eq. (134), all with the same real part, $\Lambda^{\alpha n}\left(q_{j}\right)$. For $\alpha=s$ and $\alpha=\eta$ those are the spin and charge, respectively, $\alpha n$-strings [132]. As for the spin-1/2 XXX chain [131], for a large finite system some of the 1D Hubbard model $\alpha n$-strings deviate from their TBA ideal form, Eq. (134). The effects of such string deviations [132] are in the TL though not important for the properties considered in this paper.

As in the case of the simpler models also reviewed in it, the discrete quantum numbers $q_{j}$ in Eq. (131) play the role of $\beta=c, \alpha n$ band momentum values. Consistently, the momentum eigenvalues are additive in $q_{j}$ and read,

$$
P=\sum_{j=1}^{L} q_{j} N_{c}\left(q_{j}\right)+\sum_{n=1}^{\infty} \sum_{j=1}^{L_{s n}} q_{j} N_{s n}\left(q_{j}\right)+\sum_{n=1}^{\infty} \sum_{j=1}^{L_{\eta n}}\left(\pi-q_{j}\right) N_{\eta n}\left(q_{j}\right)+\pi L_{\eta,-1 / 2} .
$$

The momentum contribution $\pi L_{\eta,-1 / 2}=\pi\left(M_{\eta}+M_{\eta,-1 / 2}\right)$ involves the number $L_{\eta,-1 / 2}$ of $\eta$-spins of projection $-1 / 2$, Eq. (127) for $\alpha=\eta$. Such a contribution follows from the paired and unpaired spins $1 / 2$ and $\eta$-spins $1 / 2$ of projection $\pm 1 / 2$ having an intrinsic momentum given by,

$$
q_{s, \pm 1 / 2}=q_{\eta,+1 / 2}=0 \text { and } q_{\eta,-1 / 2}=\pi .
$$

The set $j=1, \ldots, L_{\beta}$ of $\beta=c, \alpha n$ bands discrete momentum values $q_{j}$ belong to well-defined domains, $q_{j} \in\left[q_{\beta}^{-}, q_{\beta}^{+}\right]$. The limiting momenta $q_{\beta}^{ \pm}$appearing here are given in Eq. (B.4) of Appendix B.

The momentum and energy spectra, Eq. (135) and Eq. (B.5) of Appendix B, apply to all $4^{L}$ energy eigenstates $\left\{\left|l_{\mathrm{r}}, l_{\eta \bar{s}}, u\right\rangle\right\}$, Eq. (110). Their label $l_{\mathrm{r}}$ can now be defined. It corresponds to a short notation for the following set of TBA quantum numbers,

$$
l_{\mathrm{r}}=\left\{I_{j}^{\beta}\right\} \text { such that } N_{\beta}\left(q_{j}\right)=N_{\beta}\left([2 \pi / L] I_{j}^{\beta}\right)=1 \text { for } j=1, \ldots, L_{\beta}, \quad \beta=c, \eta n, \text { sn and } n=1, \ldots, \infty \text {. }
$$

The TBA equations, Eqs. (128) and (129), refer explicitly to LWSs. However, they can be extended to non-LWSs, Eq. (110) $[118,119,253]$. This can be achieved by formally setting some of the rapidities $\Lambda^{\eta n}$ and $\Lambda^{\text {sn }}$ in such equations equal to infinity [118,119]. For example, Eqs. (3.23b) and (3.24b) of Ref. [118] describe a $\eta$-spin non-LWS with numbers $S_{\eta}=1$ and $S_{\eta}^{z}=0$. Moreover, Eqs. (3.23a) and (3.24a) of Ref. [118] describe a LWS with numbers $S_{\eta}=S_{\eta}^{z}=0$, Eq. (110). Alternatively, in Eq. (110) one has combined symmetry with the BA solution to generate the non-LWSs from the LWSs [243].

\subsection{The composite $\alpha$ n pseudoparticles associated with the paired $\eta$-spins $(\alpha=\eta)$ and paired $\operatorname{spins}(\alpha=s)$}

It was confirmed in Refs. [243-245] that the TBA quantum number configurations combined with the spin and $\eta$-spin $S U(2)$ multiplet configurations generate the $4^{L}$ energy eigenstates that span the $1 \mathrm{D}$ Hubbard model Hilbert space. Beyond the analysis of Refs. [243-245], the Hilbert-space dimension $4^{L}$ also equals the number of independent state representations of the $1 \mathrm{D}$ Hubbard model global $[S U(2) \otimes S U(2) \otimes U(1)] / Z_{2}^{2}$ symmetry. (In 1991 and 1992 only the $S O(4)$ symmetry in the $u>0$ model global [SO(4) $\otimes U(1)] / Z_{2}$ symmetry [147] was known [243-245].)

The proof involves the requirement addressed in Appendix $C$ that in any $\operatorname{spin}(\alpha=s)$ and $\eta$-spin $(\alpha=\eta) S_{\alpha}$-fixed subspace the number of independent $\alpha$-singlet configurations $\mathcal{N}_{\text {singlet }}\left(S_{\alpha}\right)$ is exactly the same when obtained from the counting of two apparently different types of configurations. (This is similar to the spin configurations of the XXX chain.) The first type of configurations refers to the two $\alpha=\eta, s S U(2)$ group states representations associated with the spins $1 / 2(\alpha=s)$ and $\eta$-spins $1 / 2(\alpha=\eta)$ independent configurations with the same spin and $\eta$-spin, respectively, $S_{\alpha}$, Eq. (C.4) of Appendix C. The 
second type of configurations corresponds to the independent $n=1, \ldots, \infty$ bands $\left\{q_{j}\right\}$ occupancy configurations of the sets of $N_{\alpha n} \alpha n$-strings obeying the $\alpha=\eta, s$ TBA sum rules $\sum_{n=1}^{\infty} n N_{\alpha n}=\left(L_{\alpha}-2 S_{\alpha}\right) / 2$.

It follows that the set of $\alpha n$-strings of an energy and momentum eigenstate is directly related to the set of $\Pi_{\alpha} \operatorname{spin}(\alpha=s)$ and $\eta$-spin $(\alpha=\eta) \alpha$-singlet pairs, Eq. (125). (This is as for the spin-singlet pairs of the spin-1/2 XXX chain.) Such pairs involve the subset of $2 \Pi_{\alpha}=L_{\alpha}-2 S_{\alpha}$ spins $1 / 2$ and $\eta$-spins $1 / 2$, respectively, that participate in $\alpha$-singlet configurations. Specifically, each $\alpha n$-string refers to an $\alpha$-pairs configuration within which a number $n>1$ of $\alpha$-singlet pairs are bound. For $n>1$ such a binding is associated with the corresponding imaginary parts, $i(n+1-2 l) u$, of the $l=1, \ldots, n$ rapidities, $\Lambda^{\alpha n, l}\left(q_{j}\right)=\Lambda^{\alpha n}\left(q_{j}\right)+i(n+1-2 l) u$, Eq. (134), with the same real part, $\Lambda^{\alpha n}\left(q_{j}\right)$. For $n=1$ an $\alpha n$-string involves a single $\alpha$-singlet pair.

The $n>1 \alpha$-singlet pairs that are bound within an $\alpha n$-pairs configuration associated with a string of length $n>1$ are here called bound spin-singlet pairs $(\alpha=s)$ and bound $\eta$-spin-singlet pairs $(\alpha=\eta)$. For $n=1$ the rapidity $\Lambda^{\alpha 1,1}\left(q_{j}\right)$ imaginary part vanishes because a $n=1 \alpha n$-string reduces to a single $\alpha$-singlet pair. The unbound spin-singlet pairs $(\alpha=s)$ and unbound $\eta$-spin-singlet pairs $(\alpha=\eta)$ of an energy eigenstate are the $N_{\alpha 1} \alpha$-singlet pairs that refer to the $N_{\alpha 1} n=1 \alpha n$-pairs configurations.

The numbers $\Pi_{\alpha}=\left(L_{\alpha}-2 S_{\alpha}\right) / 2$ of $\operatorname{spin}(\alpha=s)$ and $\eta$-spin $(\alpha=\eta) \alpha$-singlet pairs classify the $u>0$ energy and momentum eigenstates associated with the BA solution in two different yet related and complementary ways. On the one hand, since $L_{\eta}=L-N_{c}$ and $L_{s}=N_{c}$, they are amid the quantum numbers of that solution. This follows from $N_{c}$, the spin $S_{s}$, and the $\eta$-spin $S_{\eta}$ being good quantum numbers that classify the corresponding $u>0$ energy and momentum eigenstates. On the other hand, each of the numbers $l=1, \ldots, n$ and the number $n$ that classify a TBA $\alpha n$-string and corresponding set of $l=1, \ldots, n$ rapidities, $\Lambda^{\alpha n, l}\left(q_{j}\right)=\Lambda^{\alpha n}\left(q_{j}\right)+i(n+1-2 l) u$, Eq. (134), with the same real part, $\Lambda^{\alpha n}\left(q_{j}\right)$, refer to one such pairs and to their number, respectively.

The (above mentioned) following exact TBA sum rules hold for all $u>0$ energy and momentum eigenstates,

$$
\begin{aligned}
\Pi_{\alpha} & =\sum_{n=1}^{\infty} n N_{\alpha n}=\frac{1}{2}\left(L_{\alpha}-2 S_{\alpha}\right) \text { where } \alpha=s, \eta, \\
\Pi^{S U(2)} & \equiv \sum_{\alpha=\eta, s} \Pi_{\alpha}=\sum_{\alpha=\eta, s} \sum_{n=1}^{\infty} n N_{\alpha n}=\frac{1}{2}\left(L-2 S_{s}-2 S_{\eta}\right) .
\end{aligned}
$$

This is consistent with the relation of the set of $\sum_{n=1}^{\infty} n N_{\alpha n}$ TBA $\alpha n$-strings of all lengths $n=1, \ldots, \infty$ of such a state to the set of $\Pi_{\alpha} \operatorname{spin}(\alpha=s)$ and $\eta$-spin $(\alpha=\eta) \alpha$-singlet pairs.

$\Pi^{S U(2)}$ denotes in Eq. (138) the total number of both spins and $\eta$-spins singlet pairs and $N_{\alpha n}$ is the number of $\alpha n$-pairs configurations that equals that of $\alpha n$-band discrete momentum values $q_{j}$ that are occupied. Below in Section 6.3 it is shown that the configuration of the two spins within one such unbound spin-singlet pair and that of the two $\eta$-spins within one unbound $\eta$-spin-singlet pair has a binding and anti-binding character, respectively. This applies to the internal structure of all $\Pi_{s}$ spin-singlet pairs and all $\Pi_{\eta} \eta$-spin-singlet pairs, respectively, of a $u>0$ energy and momentum eigenstate.

There is a one-to-one correspondence between the $N_{\alpha n} \alpha n$-pairs configurations with the same number $n$ of $\alpha$-singlet pairs of an energy eigenstate and the $N_{\alpha n}$ occupied momentum values $q_{j}$ of the corresponding $\alpha n$-band distribution $N_{\alpha n}\left(q_{j}\right)$, respectively. An $\alpha n$-pairs configuration involves a set of $2 n$ paired $\eta$-spins $1 / 2(\alpha=\eta)$ or paired spins $1 / 2(\alpha=s)$. They singly occupy a set of $2 n$ original-lattice sites. The use of the TBA equations given in the previous section reveals that their center of mass moves with momentum $q_{j}$. All the $2 n$ paired spins $1 / 2(\alpha=s)$ or paired $\eta$-spins $1 / 2(\alpha=\eta)$ move coherently along with it. This occurs through processes within which such $2 n$ paired spins $1 / 2$ or paired $\eta$-spins $1 / 2$ interchange position with the $M_{\alpha}=2 S_{\alpha}$ unpaired spins $1 / 2$ or unpaired $\eta$-spins $1 / 2$, respectively. Between each such a elementary process, both the latter and the $2 n$ paired $\eta$-spins $1 / 2(\alpha=\eta)$ or paired spins $1 / 2(\alpha=s)$ singly occupy original-lattice sites.

We associate one $\alpha n$ pseudoparticle and one $\alpha n$-band hole with each of the $N_{\alpha n}$ occupied and $N_{\alpha n}^{h}$ unoccupied momentum values $q_{j}$, respectively, of an $u>0$ energy eigenstate $\alpha n$-band. Such composite $\alpha n$ pseudoparticles are well defined for $u>0$ within the TL to which the TBA applies. The TL ensures that the problems concerning the $\alpha n$ pseudoparticle internal degrees of freedom and translational degrees of freedom, respectively, separate.

On the one hand, the internal degrees of freedom of a composite $\alpha n$ pseudoparticle refer to an $\alpha n$-pairs configuration. Hence there is one $\alpha n$ pseudoparticle for each $\alpha n$-pairs configuration and corresponding BA roots that involve a group of $l=1, \ldots, n$ rapidities with the same real part, Eq. (134). If $n>1$ the composite $\alpha n$ pseudoparticle has $n=2, \ldots, \infty$ $\alpha$-singlet pairs bound within it. If $n=1$ its internal degrees of freedom correspond to a single unbound $\alpha$-singlet pair.

On the other hand, the momentum $q_{j}$, Eq. (131), of an $\alpha n$ pseudoparticle refers to its translational degrees of freedom. It is associated with its center of mass motion. The set of $N_{\alpha}=\sum_{n=1}^{\infty} N_{\alpha n} \alpha n$ pseudoparticles, each carrying a momentum $q_{j}$, of a given energy eigenstate determine such a state momentum eigenvalue, as given in Eq. (135). That the $\eta n$ pseudoparticles contribution reads $\left(\pi-q_{j}\right)$ rather than $q_{j}$, follows from the configuration of the two $\eta$-spins in each $\eta$-spin-singlet pair having an anti-binding character, as confirmed below in Section 6.3.

The $l=1, \ldots, n \alpha$-singlet pairs within each of the $N_{\alpha n} \alpha n$ pseudoparticles that populate the $n=1, \ldots, \infty \alpha n$-bands of an energy and momentum eigenstate have spin $(\alpha=s)$ and $\eta$-spin $(\alpha=\eta)$ zero. The corresponding composite $\alpha n$ pseudoparticles are thus neutral particles. The $c$ pseudoparticles have in turn no internal degrees of freedom. Their occupancy configurations generate the state representations of the $c$ lattice $U(1)$ symmetry. It is independent from the model two $S U(2)$ 
symmetries. Hence the energy eigenstates spin and spin projection $(\alpha=s)$ and $\eta$-spin and $\eta$-spin projection $(\alpha=\eta), S_{\alpha}$ and $S_{\alpha}^{z}$, are determined solely by their numbers of unpaired spins $1 / 2$ and unpaired $\eta$-spins $1 / 2$ of projections $\pm 1 / 2$. Specifically, $S_{\alpha}=\left(M_{\alpha,+1 / 2}+M_{\alpha,-1 / 2}\right) / 2=M_{\alpha} / 2$ and $S_{\alpha}^{z}=-\left(M_{\alpha,+1 / 2}-M_{\alpha,-1 / 2}\right) / 2$, respectively.

As for the spin-1/2 XXX chain, there is a number of $\alpha n$ pseudoparticles sum rule. It is related to that of $\alpha$-singlet pairs, Eq. (138). The latter sum rule implies that $N_{\alpha 1}=L_{\alpha} / 2-S_{\alpha}-\sum_{n=2}^{\infty} n N_{\alpha n}$. From the use of this relation in the overall number of $\alpha n$ pseudoparticles expression, $N_{\alpha}=\sum_{n=1}^{\infty} N_{\alpha n}$, one confirms that the following sum rules are obeyed,

$$
\begin{aligned}
N_{s} & =\sum_{n=1}^{\infty} N_{s n}=\frac{1}{2}\left(N_{c}-N_{s 1}^{h}\right) \text { and } N_{\eta}=\sum_{n=1}^{\infty} N_{\eta n}=\frac{1}{2}\left(N_{c}^{h}-N_{\eta 1}^{h}\right), \\
N^{S U(2)} & =\sum_{\alpha=\eta, S} N_{\alpha}=\sum_{\alpha=\eta, S} \sum_{n=1}^{\infty} N_{\alpha n}=\frac{1}{2}\left(L-N_{s 1}^{h}-N_{\eta 1}^{h}\right) .
\end{aligned}
$$

Here $N_{\alpha 1}^{h}$ is the number of $\alpha 1$-band holes, Eq. (130) for $\alpha=\eta, s$ and $n=1 . N^{S U(2)}=\sum_{\alpha=\eta, s} N_{\alpha}$ is that in Eq. (133).

In contrast to the spin $1 / 2-X X X$ chain, the imaginary parts, $i(n+1-2 l) u$, of each set of $l=2, \ldots, n$ rapidities with the same real part depend on the interaction $u=U / 4 t$ and thus vanish as $u \rightarrow 0$. Such a set of $l=2, \ldots, n$ rapidities describes $n>1 \alpha$-singlet pairs bound within an $\alpha n$-pairs configuration. The vanishing of such rapidities imaginary parts thus gives rise to the unbinding of all $\alpha$-singlet pairs. One finds that the two $\eta$-spins $1 / 2(\alpha=\eta)$ or spins $1 / 2(\alpha=s)$ of each pair remain contributing to singlet configurations, yet each carries an independent virtual elementary charge or spin current, respectively. This reveals that the corresponding composite $\alpha$ n pseudoparticles are only well defined for $u>0$. Such a unbinding marks for finite transfer integral $t$ the qualitatively different physics of the $U=0$ and $U>0$ quantum problems, respectively. It is associated with the rearrangement of the $\eta$-spin and spin degrees of freedom in terms of the noninteracting electrons occupancy configurations that generate the finite- $t$ and $U=0$ energy and momentum eigenstates.

In an extended Takahashi subspace as defined in Appendix G, the numbers of discrete momentum values $L_{\alpha n}$ in Eq. (130) of all $\alpha n$-bands for which $N_{\alpha n}>0$ remain fixed. For the 1D Hubbard model in such subspaces one associates each $j=1, \ldots, L_{\alpha n}$ momentum values $q_{j} \alpha n$-band, Eq. (131), with a corresponding squeezed $\alpha n$ effective lattice with $j=1, \ldots, L_{\alpha n}$ sites and length $L$. Provided that the ratio $L_{\alpha n} / L$ remains finite as $L \rightarrow \infty$, in the TL such squeezed $\alpha n$ effective lattices can be represented by 1D lattices. Their spacing corresponds to the extended Takahashi subspace average distance of their $L_{\alpha n}$ sites,

$$
a_{\alpha n}=\frac{L}{L_{\alpha n}}=\frac{L}{L_{\alpha n}} a=\frac{L_{\alpha}}{L_{\alpha n}} a_{\alpha} \text { where } \alpha=\eta, s \text { and } n=1, \ldots, \infty .
$$

Therefore, the $\alpha n$ effective lattice length equals that of the original lattice. The corresponding sites then have spatial coordinates, $a_{\alpha n} j$, where $j=1, \ldots, L_{\alpha n}$. Each composite $\alpha n$ pseudoparticle singly occupied site of the $\eta n$ (and $s n$ ) effective lattice describes an $\eta$-spin-singlet (or spin-singlet) occupancy configuration. It involves a set of $2 n$ paired $\eta$-spins $1 / 2$ (or $2 n$ paired spins $1 / 2$ ) on $2 n=2, \ldots, \infty$ sites of the original lattice.

For the PDT, only the $c$ and $s 1$ pseudoparticle and corresponding $c$ and $s 1$ pseudofermion operator algebras are explicitly needed. Hence for simplicity we limit our present analysis to the $\alpha n=s 1$ pseudoparticle operator algebra. The corresponding operator representation is valid for the $1 \mathrm{D}$ Hubbard model in fixed- $L_{s 1}$ extended Takahashi subspaces. In such subspaces the local $s 1$ pseudoparticle operators obey a fermionic algebra,

$$
\left\{f_{j, s 1}^{\dagger}, f_{j^{\prime}, s 1}\right\}=\delta_{j, j^{\prime}} \text { and }\left\{f_{j, s 1}^{\dagger}, f_{j^{\prime}, s 1}^{\dagger}\right\}=\left\{f_{j, s 1}, f_{j^{\prime}, s 1}\right\}=0 .
$$

This can be confirmed in terms of their statistical interactions [262]. Such a problem is addressed in Appendix G. (Consistently, the TBA $\beta=c, s 1$ band momentum value $q_{j}$ have only occupancies zero and one.) Each of the $N_{s 1}$ occupied $s 1$ effective lattice sites corresponds to a spin-singlet pair. It involves two original lattice sites occupied by two paired spins $1 / 2$ of opposite spin projection.

The $s 1$ pseudoparticle translational degrees of freedom center of mass motion are described by operators $f_{j, s 1}^{\dagger}$ (and $f_{j, s 1}$ ). They create (and annihilate) one $s 1$ pseudoparticle at the $s 1$ effective lattice site $x_{j}=a_{s 1} j$. Here $j=1, \ldots, L_{s 1}$ and $L_{s 1}$ is given in Eq. (130) for $\alpha n=s 1$. This is as for the local creation and annihilation $c$ pseudoparticle operators, Eq. (121).

The $\beta=c, s 1$ pseudoparticle operators labeled by the corresponding $\beta=c, s 1$ bands $j=1, \ldots, L_{\beta}$ momentum values $q_{j}$ defined in Eqs. (131) and (132) then read,

$$
f_{q_{j}, \beta}^{\dagger}=\frac{1}{\sqrt{L}} \sum_{j^{\prime}=1}^{L_{\beta}} e^{i q_{j} x_{j^{\prime}}} f_{j^{\prime}, \beta}^{\dagger} \text { and } f_{q_{j}, \beta}=\left(f_{q_{j}, \beta}^{\dagger}\right)^{\dagger} \text { where } j=1, \ldots, L_{\beta} \text { and } \beta=c, s 1 \text {. }
$$

Such momentum values $q_{j}$ are the quantum numbers of the exact BA solution whose occupancy configurations generate the $u>0$ energy and momentum eigenstates,

Besides acting within fixed- $L_{s 1}$ extended Takahashi subspaces, the $s 1$ pseudoparticle operators labeled by momentum $q_{j}$ also appear in the expressions of the shake-up effects generators. Such generators transform extended Takahashi subspaces quantum number values into each other. 


\subsection{Charge (and spin) current carriers and the general c, $\eta n$ bands (and sn bands) hole representation}

The relation of the composite $\alpha n$ pseudoparticles to the paired spins $1 / 2(\alpha=s)$ and paired $\eta$-spins $1 / 2(\alpha=\eta)$ was the problem revisited in Section 5.2. A related issue whose clarification is needed for the study of the charge and spin currents and their carriers is addressed in this section. It refers to the relation of the set of holes in each $\alpha n$-band populated by $\alpha n$ pseudoparticles to the spins $1 / 2(\alpha=s)$ and $\eta$-spins $1 / 2(\alpha=\eta)$. There are $N_{\alpha n}^{h}=2 S_{\alpha}+\sum_{n^{\prime}=n+1}^{\infty} 2\left(n^{\prime}-n\right) N_{\alpha n^{\prime}}$ such holes, Eq. (130), in each $\alpha n$-band.

The 1D Hubbard model in a uniform vector potential $\Phi / L$ whose Hamiltonian is given in Eq. (4) of Ref. [263] remains solvable by the BA. Its coupling to the charge/ $\eta$-spin and spin degrees of freedom the flux $\Phi$ reads $\Phi=\Phi_{\uparrow}=\Phi_{\downarrow}$ and $\Phi=\Phi_{\uparrow}=-\Phi_{\downarrow}$, respectively [263,264]. The LWSs momentum eigenvalues, $P\left(\Phi_{\uparrow}, \Phi_{\downarrow}\right)$, have the general form [264],

$$
\begin{aligned}
P(\Phi / L)=P\left(\Phi_{\uparrow} / L, \Phi_{\downarrow} / L\right) & =P(0)+\left(N_{c}-\sum_{n} 2 n N_{s n}\right) \frac{\Phi_{\uparrow}-\Phi_{\downarrow}}{2 L}-\left(N_{c}^{h}-\sum_{n} 2 n N_{\eta n}\right) \frac{\Phi_{\uparrow}+\Phi_{\downarrow}}{2 L} \\
& =P(0)+2 S_{s} \frac{\Phi_{\uparrow}-\Phi_{\downarrow}}{2 L}-2 S_{\eta} \frac{\Phi_{\uparrow}+\Phi_{\downarrow}}{2 L} .
\end{aligned}
$$

The LWSs $\Phi=0$ momentum eigenvalue $P(0)$ appearing here is that in Eq. (135) for $L_{\eta,-1 / 2}=0$.

The TBA equations for the model in a uniform vector potential are given in Eq. (9) of Ref. [263]. The only difference relative to the $\Phi=0$ case, is that the $c$ band, $s n$ band, and $\eta n$ band momentum values $q_{j}$ are replaced by $q_{j}+\Phi_{\uparrow} / L, q_{j}-n\left(\Phi_{\uparrow}-\Phi_{\downarrow}\right) / L$, and $q_{j}-n\left(\Phi_{\uparrow}+\Phi_{\downarrow}\right) / L$, respectively. Hence concerning the coupling to the (i) charge $/ \eta$-spin and (ii) spin degrees of freedom, this gives (i) $q_{j}+\Phi / L, q_{j}$, and $q_{j}-2 n \Phi / L$ and (ii) $q_{j}+\Phi / L, q_{j}-2 n \Phi / L$, and $q_{j}$, respectively.

The $\alpha=\eta$ and $\alpha=s$ current operators expectation values of the $\Phi \rightarrow 0$ LWSs can then be derived from the $\Phi / L$ dependence of the energy eigenvalues $E(\Phi / L)$. Specifically, $\left\langle\hat{J}_{\eta}^{z}\right\rangle=d E(\Phi / L) /\left.d(\Phi / L)\right|_{\Phi=\Phi_{\uparrow}=\Phi_{\downarrow}=0}$ and $\left\langle\hat{J}_{s}^{z}\right\rangle=$ $d E(\Phi / L) /\left.d(\Phi / L)\right|_{\Phi=\Phi_{\uparrow}=-\Phi_{\downarrow}=0}$, respectively. Moreover, $d P(\Phi / L) /\left.d(\Phi / L)\right|_{\Phi=\Phi_{\uparrow}=\Phi_{\downarrow}=0}$ gives the number of charge $/ \eta$-spin carriers and $d P(\Phi / L) /\left.d(\Phi / L)\right|_{\Phi=\Phi_{\uparrow}-\Phi_{\downarrow}=0}$ that of spin carriers that couple to the vector potential $\Phi / L$. The use of the exact momentum eigenvalues, Eq. (143), then reveals that such carriers are the $M_{\eta}=2 S_{\eta}$ unpaired $\eta$-spins $1 / 2$ and $M_{s}=2 S_{s}$ unpaired spins $1 / 2$, respectively. (This is as for the unpaired spins of the spin- $1 / 2$ chain $X X X$ chain.)

It is thus useful to consider the unpaired $\eta$-spins $1 / 2(\alpha=\eta)$ and unpaired spins $1 / 2(\alpha=s)$ densities $m_{S_{\alpha}} \equiv 2 S_{\alpha} / L=$ $M_{\alpha} / L$. The energy and momentum eigenstates that span the subspaces with fixed values for the $\alpha=\eta, s$ numbers $L_{\alpha}$, Eq. (123), have $S_{\alpha}$ values in the range $S_{\alpha} \in\left[0, L_{\alpha} / 2\right]$. Hence, for the corresponding unpaired $\eta$-spins $1 / 2$ and unpaired spins densities this gives $m_{S_{\eta}} \in\left[0, n_{c}^{h} / 2\right]$ and $m_{S_{s}} \in\left[0, n_{c} / 2\right]$, respectively.

In the $u \rightarrow \infty$ limit, one explicitly confirms that up to first order in $\Phi / L$ the dependence of the energy eigenvalues $E(\Phi / L)$ on $\Phi / L$ can be expressed in terms of a dependence on $\left(m_{S_{\alpha}} \Phi\right) / L$. It refers to $\left(m_{S_{\eta}} \Phi\right) / L$ for $\Phi=\Phi_{\uparrow}=\Phi_{\downarrow}[103]$ and $\left(m_{S_{s}} \Phi\right) / L$ for $\Phi=\Phi_{\uparrow}=-\Phi_{\downarrow}$. This ensures that for all LWSs $\left|l_{\mathrm{r}}, l_{\eta s}^{0}, \infty\right\rangle$ the above $\alpha=\eta$, $s$ currents $\left\langle\hat{J}_{\alpha}^{z}\right\rangle$ have an overall factor $m_{S_{\alpha}}=2 S_{\alpha} / L$. (In Ref. [103] it was considered that in the $\alpha=\eta$ case the corresponding total exact flux $2 S_{\eta} \Phi$ was shared by the $N_{c}^{h}$ holes in the $c$ band whose number equals that of $\eta$-spins $1 / 2, L_{\eta}=N_{c}^{h}$. This gives $2 S_{\eta} \Phi=N_{c}^{h} \Phi^{e f f}$ and thus $\left.\Phi^{e f f}=\left[2 S_{\eta} / N_{c}^{h}\right] \Phi.\right)$

Combination of these $u \rightarrow \infty$ properties with the invariance under the electron-rotated-electron unitary transformation of the $M_{\eta}=2 S_{\eta}$ unpaired $\eta$-spins $1 / 2$ and $M_{s}=2 S_{s}$ unpaired spins $1 / 2$ provides useful physical information. That invariance implies that for $u>0$ the unpaired $\eta$-spins $1 / 2$ and unpaired spins $1 / 2$ that populate the states $\left|l_{\mathrm{r}}, l_{\eta s}^{0}, u\right\rangle=\hat{V}^{\dagger}\left|l_{\mathrm{r}}, l_{\eta s}^{0}, \infty\right\rangle$ have properties similar to those that in the $u \rightarrow \infty$ limit populate the corresponding states $\left|l_{\mathrm{r}}, l_{\eta s}, \infty\right\rangle$ belonging the same $V(u)$-set of states. This reveals that in the case of coupling to charge $/ \eta$-spin $(\alpha=\eta)$ and spin $(\alpha=s)$ the energy eigenvalues $E(\Phi / L)$ dependence on $\Phi / L$ is up to first order in $\Phi / L$ of the general form $C_{u}\left(m_{S_{\alpha}} \Phi\right) / L$ for $u>0$. Here $C_{u}$ is some $u$, $n_{e}$, and $m$ dependent coefficient independent of $\Phi / L$. Hence the corresponding $\alpha$ current has the general form $\left\langle\hat{J}_{\eta}^{z}\right\rangle=C_{u} m_{S_{\alpha}}$ for all LWSs $\left|l_{r}, l_{\eta s}^{0}, u\right\rangle=\hat{V}^{\dagger}\left|l_{r}, l_{\eta s}^{0}, \infty\right\rangle$.

For simplicity, we denote the LWSs $\alpha=\eta, s$ currents by $\left\langle\hat{J}_{\alpha, L W S}^{z}\left(l_{\mathrm{r}}, S_{\alpha}\right)\right\rangle=\left\langle l_{\mathrm{r}}, S_{\alpha},-S_{\alpha}^{z}\left|\hat{J}_{\alpha}^{z}\right| l_{\mathrm{r}}, S_{\alpha},-S_{\alpha}^{z}\right\rangle$. Here we have implicitly incorporated in $l_{\mathrm{r}}$ the spin $S_{s}$ when $\alpha=\eta$ and the $\eta$-spin $S_{\eta}$ when $\alpha=s$. From the use of procedures similar to those leading to the spin-1/2XXX spin currents, Eq. (69), one finds that the $\alpha=\eta, s$ currents carried by the non-LWSs have the following exact relation to that of the corresponding LWS [264],

$$
\left\langle\hat{J}_{\alpha}^{z}\left(l_{\mathrm{r}}, M_{\alpha,+1 / 2}, M_{\alpha,-1 / 2}\right)\right\rangle=\frac{\left(M_{\alpha,+1 / 2}-M_{\alpha,-1 / 2}\right)}{2 S_{\alpha}}\left\langle\hat{J}_{\alpha, L W S}^{z}\left(l_{\mathrm{r}}, S_{\alpha}\right)\right\rangle \text { where } \alpha=\eta, s .
$$

The arguments already used in the case of the spin- $1 / 2 X X X$ chain, concerning the LWS currents quantum associated with $\alpha$ current changes generated by $\alpha$-flip processes, apply. One then finds that the elementary current carried by a unpaired $\eta$-spin $1 / 2(\alpha=\eta)$ of projection $\pm 1 / 2$ and unpaired spin $1 / 2$ of projection $\pm 1 / 2$ is given by,

$$
j_{\alpha, \pm 1 / 2}= \pm \frac{\left\langle\hat{J}_{\alpha, L W S}^{z}\left(l_{\mathrm{r}}, S_{\alpha}\right)\right\rangle}{2 S_{\alpha}} \text { where } \alpha=\eta, s .
$$

This is similar to Eq. (70) for that chain. 
Here we do not address the issue of the spin currents in terms of the $s n$-bands hole representation and corresponding spinon representation. Indeed, it is similar to that reported in Section 3.4 for the spin- $1 / 2 X X X$ chain. Concerning the charge $/ \eta$ spin currents, the translational degrees of freedom of the $2 S_{\eta}$ unpaired $\eta$-spins that couple to the vector potential are now described by an average number of $2 S_{\eta}$ holes both in the $c$ band and $\eta n$ bands for which $N_{\eta n}>0$ [264]. The total number of holes in such bands can be written as $N_{c}^{h}=2 S_{\eta}+\sum_{n=1}^{\infty} 2 n N_{\eta n}$ and $N_{\eta n}^{h}=2 S_{\eta}+\sum_{n^{\prime}=n+1}^{\infty} 2\left(n^{\prime}-n\right) N_{\eta n^{\prime}}$, respectively. All processes associated with the $\eta n$ bands and $\eta$-spins $1 / 2$ are similar to those described in Section 3.4 involving spin $n$ bands and spins $1 / 2$.

Some of the holon representations associate each of the $N_{c}^{h}=2 S_{\eta}+\sum_{n=1}^{\infty} 2 n N_{\eta n} c$-band holes with a $\eta$-spin $1 / 2$ holon [66,67]. Both the range of validity of that representation and its relation to the extended $c$-band hole and $\eta n$-band holes more general representation show basic similarities to the same problems for the spinon representation and extended $n$-bands hole representation revisited in Section 3.4 for the spin- $1 / 2 X X X$ chain. In the case of charge currents, this applies for instance to the description of the translational degrees of freedom of the $M_{\eta}=2 S_{\eta}$ unpaired $\eta$-spins $1 / 2$ of $u>0$ energy eigenstates [264]. Such degrees of freedom are described by an average number of $2 S_{\eta}$ holes out of both the $N_{c}^{h}=2 S_{\eta}+\sum_{n=1}^{\infty} 2 n N_{\eta n} c$-band holes and $N_{\eta n}^{h}=2 S_{\eta}+\sum_{n^{\prime}=n+1}^{\infty} 2\left(n^{\prime}-n\right) N_{\eta n^{\prime}}$ holes of $\eta n$ bands for which $N_{\eta n}>0$. However, their internal $\eta$-spin degrees of freedom cannot be associated with such $c$ - and $\eta n$-bands holes. This is similar to the spinon representation of the spin-1/2 XXX chain. For the additional information on the role of the $c$ band holes in charge transport see Ref. [264].

\section{The 1D Hubbard model pseudoparticles quantum liquid}

In this section the use of the $\beta=c, \alpha n$ pseudoparticle representation of the 1D Hubbard model to describe its low-energy physics is revisited. As in the case of the simpler models reviewed here, the $\beta=c, \alpha$ n pseudoparticle energy functional resembles that of the low-energy Fermi liquid. The 1D Hubbard model describes interacting electrons on a lattice. It is a non-perturbative quantum problem for which there are no quasiparticles, as defined in a Fermi liquid: The $\beta=c, \alpha n$ pseudoparticles do not become electrons upon turning off adiabatically the interaction $U$. The one-electron physics is thus qualitatively different from that of a Fermi liquid. However, the two-electron physics resembles that of such a liquid [89-93,104,105].

Our goal here is to apply the general $\beta=c, \alpha$ pseudoparticle energy functional introduced below in Section 6.1 to the description of the model low-energy physics in Section 6.2. However, that functional is valid at all energy scales. There is though a restriction to its applicability reported below in Section 6.1. (It basically is the same as that of the spin-1/2 XXX chain spin pseudoparticle quantum liquid.)

There are several advantages in using the $\beta=c, \alpha n$ pseudoparticle representation in the study of the low-energy properties. First, since it applies to all energy scales, its use reveals that the usual low-energy spin-charge separation results from a more general separation of the spin and charge degrees of freedom. It occurs at all energy scales and is associated with the spin $S U(2)$ and charge $U(2)=S U(2) \otimes U(1)$ symmetries, respectively, in the model $[S U(2) \otimes S U(2) \otimes U(1)] / Z_{2}^{2}$ global symmetry. Second, all low-energy two-particle quantities are controlled by simple $\beta$ pseudoparticles zero-momentum forward-scattering interactions. As in a Fermi liquid, those are associated with $f$ function terms in the theory energy functional. Hence all such quantities can be computed by the familiar methods of a Fermi liquid. This simplifies their physical understanding. Third, the form of the $\alpha 1$ pseudoparticle energy dispersions provides valuable information on the type of pairing of the two $\eta$-spins $1 / 2(\alpha=\eta)$ and spins $1 / 2(\alpha=s)$ within each $\alpha$-singlet pair. A set of $\Pi_{\alpha}=\sum_{n=1}^{\infty} n N_{\alpha n}=$ $\left(L_{\alpha}-2 S_{\alpha}\right) / 2$ such pairs populates each $u>0$ energy and momentum eigenstate. Furthermore, under a suitable unitary transformation that shifts the $\beta$ pseudoparticle momentum values $q_{j}$, those are mapped onto $\beta$ pseudofermions in terms of which the study of the model finite-energy dynamical properties simplifies.

\subsection{The $c$ and $\alpha$ n pseudoparticle quantum liquid I: The general energy functional and its energy scales}

As in the case of the simpler models discussed in Sections 2 and 3, there is a PS for each ground state with electronic density $n_{e}$ and spin density $m$ arbitrary values. In this section we consider PSs whose ground states refer to electronic densities $n_{e} \in[0,1]$ and spin densities $m \in\left[0, n_{e}\right]$. They are thus LWSs. Their $\beta$-band pseudoparticle momentum distribution functions are given by,

$$
N_{c}^{0}\left(q_{j}\right)=\theta\left(q_{j}-q_{F c}^{-}\right) \theta\left(q_{F c}^{+}-q_{j}\right), \quad N_{s 1}^{0}\left(q_{j}\right)=\theta\left(q_{j}-q_{F s 1}^{-}\right) \theta\left(q_{F s 1}^{+}-q_{j}\right) \text { and } N_{\alpha n}^{0}\left(q_{j}\right)=0 \text { for } \alpha n \neq s 1 .
$$

The $c$ and $s 1$ bands Fermi momentum values $q_{F \beta}^{ \pm}$appearing here are given in Eqs. (C.4)-(C.11) of Ref. [105]. Ignoring $\mathcal{O}(1 / L)$ corrections within the TL simplifies the $\beta=c, s 1$ distributions, Eq. (146), to $N_{\beta}^{0}\left(q_{j}\right)=\theta\left(q_{F \beta}-\left|q_{j}\right|\right)$. Here the $\beta=c, s 1$ Fermi momentum $q_{F \beta}$ reads,

$$
q_{\mathrm{Fc}}=2 k_{F}=\pi n_{e} \text { and } q_{\mathrm{Fs} 1}=k_{F \downarrow}=\pi n_{e \downarrow} .
$$

Hence the ground states under consideration are neither populated by composite $s n$ pseudoparticles with $n>1$ spin-singlet pairs nor by composite $\eta n$ pseudoparticles with any number $n=1, \ldots, \infty$ of $\eta$-spin-singlet pairs. Since they are LWSs, they 
have no unpaired spins of projection $-1 / 2$ and no unpaired $\eta$-spins of projection $-1 / 2$. Their $M_{\eta}=2 S_{\eta}=-2 S_{\eta}^{z}=L-N_{e}$ unpaired $\eta$-spins and $M_{s}=2 S_{s}=-2 S_{s}^{z}=N_{e \uparrow}-N_{e \downarrow}$ unpaired spins have all projection $+1 / 2$.

The PS excited states $\beta$-bands distribution functions are of the general form $N_{\beta}\left(q_{j}\right)=N_{\beta}^{0}\left(q_{j}\right)+\delta N_{\beta}\left(q_{j}\right)$. Here $\beta=c, \alpha n$, $\alpha=\eta, s$, and $n=1, \ldots, \infty$. In the specific case of the $\beta=c, s 1$ bands, one can classify in the TL the deviations,

$$
\delta N_{\beta}\left(q_{j}\right)=N_{\beta}\left(q_{j}\right)-N_{\beta}^{0}\left(q_{j}\right) \text { for } j=1, \ldots, L_{\beta},
$$

as $\delta N_{\beta}^{F}\left(q_{j}\right)$ and $\delta N_{\beta}^{N F}\left(q_{j}\right)$, respectively. On the one hand, for the deviations $\delta N_{\beta}^{F}\left(q_{j}\right)$ the band momentum $q_{j}$ is such that $\lim _{L \rightarrow \infty}\left(\left|q_{j}\right|-q_{F \beta}\right)=0$. On the other hand, in the case of $\delta N_{\beta}^{N F}\left(q_{j}\right)$ the momentum difference $\lim _{L \rightarrow \infty}\left(\left|q_{j}\right|-q_{F \beta}\right)$ remains finite in the TL. For the excited states belonging to a PS, one as that $\sum_{\beta=c, s 1} \sum_{j=1}^{L_{\beta}}\left|\delta N_{\beta}^{N F}\left(q_{j}\right)\right| / L \rightarrow 0, \sum_{n=1}^{\infty} \sum_{j=1}^{L_{\eta n}}\left|\delta N_{\eta n}\left(q_{j}\right)\right| / L \rightarrow 0$, $\sum_{n=2}^{\infty} \sum_{j=1}^{L_{s n}}\left|\delta N_{s n}\left(q_{j}\right)\right| / L \rightarrow 0, \delta S_{s} / L \rightarrow 0$, and $\delta S_{\eta} / L \rightarrow 0$ as $L \rightarrow \infty$. For a PS there are though no restrictions on the value of the excitation energy and excitation momentum.

It is often convenient within the TL to replace the $\beta=c, \alpha$ band discrete momentum values $q_{j}$, Eq. (131), such that $q_{j+1}-q_{j}=2 \pi / L$, by a corresponding continuous momentum variable, $q$. It belongs to a domain $q \in\left[q_{\beta}^{-}, q_{\beta}^{+}\right]$whose limiting momentum values $q_{\beta}^{ \pm}$are given in Eq. (B.4) of Appendix B. Ignoring again $\mathcal{O}(1 / L)$ corrections, one finds that $q_{\beta}^{ \pm} \approx \pm q_{\beta}$. For the present PSs whose ground states are LWSs the limiting momenta $q_{\beta}$ then read,

$$
q_{c}=\pi, \quad q_{s 1}=k_{F \uparrow}, \quad q_{s n}=\left(k_{F \uparrow}-k_{F \downarrow}\right)=\pi m \text { and } q_{\eta n}=\left(\pi-2 k_{F}\right)=\pi\left(1-n_{e}\right) .
$$

Within the continuum momentum $q$ representation, the deviation values $\delta N_{\beta}\left(q_{j}\right)=-1$ and $\delta N_{\beta}\left(q_{j}\right)=+1$ in Eq. (148) become $\delta N_{\beta}(q)=-(2 \pi / L) \delta\left(q-q_{j}\right)$ and $\delta N_{\beta}(q)=+(2 \pi / L) \delta\left(q-q_{j}\right)$, respectively. According to Eqs. (131) and (132), under a transition to an excited state, the $\beta$ band discrete momentum values $q_{j}=(2 \pi / L) I_{j}^{\beta}$ may undergo a collective shift, $(2 \pi / L) \Phi_{\beta}^{0}= \pm \pi / L$. Here $\Phi_{\beta}^{0}$ reads,

$$
\begin{aligned}
& \Phi_{c}^{0}=0 \text { for } \delta N^{S U(2)} \text { even and } \Phi_{c}^{0}= \pm \frac{1}{2} \text { for } \delta N^{S U(2)} \text { odd; } \\
& \Phi_{\alpha n}^{0}=0 \text { for } \delta N_{c}+\delta N_{\alpha n} \text { even and } \Phi_{\alpha n}^{0}= \pm \frac{1}{2} \text { for } \delta N_{c}+\delta N_{\alpha n} \text { odd, }
\end{aligned}
$$

where $\alpha=\eta, s$ and $n=1, \ldots, \infty . \delta N^{S U(2)}$ is in this equation the deviation in the number $N^{S U(2)}$ in Eq. (133). For $q$ at the $\beta=c, s 1$ and $\iota= \pm$ Fermi points, $\iota q_{F \beta}$, such a shake-up effect is captured within the continuum representation by additional deviations, $\pm(\pi / L) \delta\left(q-\iota q_{F \beta}\right)$. For transitions to an excited state for which $\delta L_{\alpha n} \neq 0$, the removal or addition of BA $\alpha n$ band discrete momentum values occurs in the vicinity of the band edges $q_{\alpha n}^{-}=-q_{\alpha n}^{+}$, Eq. (B.4) of Appendix B. Those are zero-momentum and zero-energy processes.

The PS energy functionals are derived from the use of the TBA equations, Eqs. (128)-(129), and general energy spectra, Eq. (B.5) of Appendix B. Specifically, one uses in them $\beta$-bands momentum distribution functions of form $N_{\beta}\left(q_{j}\right)=N_{\beta}^{0}\left(q_{j}\right)+$ $\delta N_{\beta}\left(q_{j}\right)$. Their deviations, Eq. (148), play an important role. The combined and consistent solution of those equations and spectra up to second order in such deviations then leads to [92,99],

$$
\delta E=\sum_{\beta} \sum_{j=1}^{L_{\beta}} \varepsilon_{\beta}\left(q_{j}\right) \delta N_{\beta}\left(q_{j}\right)+\frac{1}{L} \sum_{\beta} \sum_{\beta^{\prime}} \sum_{j=1}^{L_{\beta}} \sum_{j^{\prime}=1}^{L_{\beta^{\prime}}} \frac{1}{2} f_{\beta \beta^{\prime}}\left(q_{j}, q_{j^{\prime}}\right) \delta N_{\beta}\left(q_{j}\right) \delta N_{\beta^{\prime}}\left(q_{j^{\prime}}\right)+\sum_{\alpha=\eta, s} \varepsilon_{\alpha,-1 / 2} M_{\alpha,-1 / 2} .
$$

The $\beta=c, \alpha n$ band energy dispersions $\varepsilon_{\beta}\left(q_{j}\right)$ appearing here are given by,

$$
\varepsilon_{\beta}\left(q_{j}\right)=E_{\beta}\left(q_{j}\right)+\varepsilon_{\beta}^{c}\left(q_{j}\right) \text { and } \varepsilon_{\beta}^{c}\left(q_{j}\right)=\frac{t}{\pi} \int_{-Q}^{Q} d k 2 \pi \bar{\Phi}_{c \beta}\left(\frac{\sin k}{u}, \frac{\Lambda_{0}^{\beta}\left(q_{j}\right)}{u}\right) \sin k \text { for } j=1, \ldots, L_{\beta} .
$$

$E_{\beta}\left(q_{j}\right)$ is in this equation for $\beta=c, \eta n$, sn the energy spectrum, Eq. (B.7) of Appendix B, with the rapidity functions those of the ground state, $\Lambda_{0}^{v}\left(q_{j}\right)=\sin k_{0}^{c}\left(q_{j}\right) / u$ and $\Lambda_{0}^{\alpha n}\left(q_{j}\right)$. The latter are the solutions of Eqs. (128) and (129) for the corresponding distribution function distributions, Eq. (146). The parameter $Q$ in Eq. (152) and related parameters $B, r_{c}^{0}$, and $r_{0}^{s}$ read,

$$
Q \equiv k_{c}^{0}\left(2 k_{F}\right), \quad B \equiv \Lambda_{0}^{s 1}\left(k_{F \downarrow}\right), \quad r_{c}^{0}=\frac{\sin Q}{u} \text { and } r_{s}^{0}=\frac{B}{u} \text {. }
$$

The dressed rapidity phase shifts $2 \pi \bar{\Phi}_{c \beta}\left(r, r^{\prime}\right)$ in Eq. (152) are a particular case of the general dressed rapidity phase shifts $2 \pi \bar{\Phi}_{\beta \beta^{\prime}}\left(r, r^{\prime}\right)$. Those are defined by the integral equations given in Appendix H [99]. Such phase shifts are associated with the following corresponding dressed phase shifts expressed in terms of the $\beta$-band momentum $q_{j}$ and $\beta^{\prime}$-band momentum $q_{j^{\prime}}$,

$$
2 \pi \Phi_{\beta \beta^{\prime}}\left(q_{j}, q_{j^{\prime}}\right)=2 \pi \bar{\Phi}_{\beta \beta^{\prime}}\left(r, r^{\prime}\right) \text { where } r=\Lambda_{0}^{\beta}\left(q_{j}\right) / u \text { and } r^{\prime}=\Lambda_{0}^{\beta^{\prime}}\left(q_{j^{\prime}}\right) / u \text {. }
$$

Within the continuum $q$ representation, the $\beta$ band group velocities are given by,

$$
v_{\beta}\left(q_{j}\right)=\left.\frac{d \varepsilon_{\beta}(q)}{d q}\right|_{q=q_{j}}, \quad v_{c} \equiv v_{c}\left(q_{F c}\right)=v_{c}\left(2 k_{F}\right) \text { and } v_{s 1} \equiv v_{c}\left(q_{F s 1}\right)=v_{s 1}\left(k_{F \downarrow}\right) \text {, }
$$


where $\beta=c, \eta n, s n$ and $n=1, \ldots, \infty$. They appear in the expression of the $f$ functions in the second-order terms of the energy functional, Eq. (151), which reads [92,93],

$$
\begin{aligned}
f_{\beta \beta^{\prime}}\left(q_{j}, q_{j^{\prime}}\right) & =v_{\beta}\left(q_{j}\right) 2 \pi \Phi_{\beta \beta^{\prime}}\left(q_{j}, q_{j^{\prime}}\right)+v_{\beta^{\prime}}\left(q_{j^{\prime}}\right) 2 \pi \Phi_{\beta^{\prime} \beta}\left(q_{j^{\prime}}, q_{j}\right) \\
& +\frac{1}{2 \pi} \sum_{\beta^{\prime \prime}=c, s 1} \sum_{\iota= \pm} v_{\beta^{\prime \prime}} 2 \pi \Phi_{\beta^{\prime \prime} \beta}\left(\iota q_{F \beta^{\prime \prime}}, q_{j}\right) 2 \pi \Phi_{\beta^{\prime \prime} \beta^{\prime}}\left(\iota q_{F \beta^{\prime \prime}}, q_{j^{\prime}}\right) .
\end{aligned}
$$

The dressed momentum phase shift $2 \pi \Phi_{\beta \beta^{\prime}}\left(q_{j}, q_{j^{\prime}}\right)$ appearing here is given in Eq. (154).

The only restriction to the applicability of the $\beta=c, \alpha$ n pseudoparticle energy functional, Eq. (151), is that associated with a PS definition. It is such that within the TL the deviations $\delta N_{c}^{N F}\left(q_{j}\right), \delta N_{s 1}^{N F}\left(q_{j}\right), \delta N_{s n}\left(q_{j}\right)$ for $n=2, \ldots, \infty$, and $\delta N_{\eta n}\left(q_{j}\right)$ for $n=1, \ldots, \infty$ involve a finite number of $\beta=c, \alpha$ pseudoparticles. Hence such a restriction can be expressed as,

$$
\lim _{L \rightarrow \infty} \frac{\left(\sum_{\beta=c, s 1} \sum_{j=1}^{L_{\beta}}\left|\delta N_{\beta}^{N F}\left(q_{j}\right)\right|+\sum_{n=1}^{\infty} \sum_{j=1}^{L_{\eta n}}\left|\delta N_{\eta n}\left(q_{j}\right)\right|+\sum_{n=2}^{\infty} \sum_{j=1}^{L_{s n}}\left|\delta N_{s n}\left(q_{j}\right)\right|\right)}{L} \rightarrow 0 .
$$

The general energy spectrum, Eq. (B.5) of Appendix B, gives the energy eigenvalues. That in Eq. (151) rather provides the excited-state energy eigenvalues minus the ground state energy. The energy dispersion term $\varepsilon_{\beta}^{c}\left(q_{j}\right)$ in Eq. (152) as well as the $f$-function terms in Eq. (151) are absent from Eq. (B.5) of Appendix B. Indeed, they stem from such energies differences. This is why the expressions of the energy dispersion term $\varepsilon_{\beta}^{c}\left(q_{j}\right)$ and $f$-function involve dressed phase shifts. Those emerge under the transitions from the ground state to excited states. The one- and two-electron excited states spectra can be expressed in terms of the $\beta=c, \alpha$ energy dispersions, Eq. (152) [89].

In the particular case of the magnetic-field energy $2 \mu_{B} H=2 \mu_{B} H(m)$ and chemical potential $\mu=\mu\left(n_{e}\right)$ on the right-hand side of Eq. (151) and related energy scales, we consider extended ranges of the densities $n_{e}$ and $m$. Such important energy scales appear in the following relations between energy dispersions with different yet useful zero energy levels,

$$
\varepsilon_{c}^{0}\left(q_{j}\right)=\varepsilon_{c}\left(q_{j}\right)-\mu_{\eta}+\mu_{s} \text { and } \varepsilon_{\alpha n}^{0}\left(q_{j}\right)=\varepsilon_{\alpha n}\left(q_{j}\right)-n 2 \mu_{\alpha},
$$

where $\alpha=\eta, s$ and $n=1, \ldots, \infty$. They are uniquely determined by the energy dispersions $\varepsilon_{c}^{0}\left(q_{j}\right)$ and $\varepsilon_{s 1}^{0}\left(q_{j}\right)$ at the corresponding Fermi points as follows [89],

$$
\begin{aligned}
2 \mu_{B} H & =-\operatorname{sgn}\{m\} \varepsilon_{s 1}^{0}\left(q_{F s 1}\right), \\
\mu & =-\operatorname{sgn}\left\{\left(1-n_{e}\right)\right\}\left(\varepsilon_{c}^{0}\left(q_{F c}\right)+\frac{1}{2} \varepsilon_{s 1}^{0}\left(q_{F s 1}\right)\right) \text { for } n_{e} \neq 1 \text { and } \mu \in\left[-\mu^{0}, \mu^{0}\right] \text { for } n_{e}=1 .
\end{aligned}
$$

Note that due to the terms in the expressions in Eq. (158) involving the $\alpha=\eta$, $s$ energy scales $2 \mu_{\alpha}$, Eq. (B.6) of Appendix B, the dispersions $\varepsilon_{c}^{0}\left(q_{j}\right)$ and $\varepsilon_{\alpha n}^{0}\left(q_{j}\right)$ are actually independent of such energy scales. This can be confirmed by inspection of the form of the energy dispersion term $E_{\beta}\left(q_{j}\right)$, Eq. (B.7) of Appendix B for $\beta=c, \alpha n$, appearing in Eq. (152). The zero energy level of the $\beta=c, \alpha n$ energy dispersions $\varepsilon_{\beta}\left(q_{j}\right)$ is that of the ground state. In contrast, that of the $\beta=c, \alpha n$ energy dispersions $\varepsilon_{\beta}^{0}\left(q_{j}\right)$ refers to the BA absolute zero energy level. For the $\alpha n$ bands, the latter zero energy level is such that $\varepsilon_{\alpha n}^{0}\left(q_{\alpha n}^{ \pm}\right)=0$.

The finite- $u$ Mott-Hubbard gap $2 \mu^{0}$, Eq. (B.8) of Appendix B, finiteness implies that the chemical potential curve $\mu=\mu\left(n_{e}\right)$ has a discontinuity at $n_{e}=1$. The corresponding chemical-potential dependence on the hole concentration, $x=\left(1-n_{e}\right)$, is such that $\mu(x)=-\mu(-x)$ with $\mu \in\left[\mu^{0}, \mu^{1}\right]$ for $x \geq 0$. Here the energy scale $2 \mu^{1}$ associated with $\mu^{1}=\mp \lim _{x \rightarrow \pm 1} \mu(x)$ reads $2 \mu^{1}=U+4 t$. For $u \gg 1$ and $m=0$ the chemical-potential curve $\mu=\mu(x)$ behaves as $2 \mu(x)=\operatorname{sgn}\{x\}(U-4 t \cos (\pi x))$ for both $x \in[-1,0]$ and $x \in[0,1]$ and $2 \mu(0) \in[-(U-4 t),(U-4 t)]$ at $x=0$. Furthermore, the magnetic energy scale $2 \mu_{B} H$ dependence on the spin density $m$ is such that $2 \mu_{B} H(m)=-2 \mu_{B} H(-m)$ with $2 \mu_{B}|H(m)| \in\left[0,2 \mu_{B} H_{c}\right]$ for $m \in[-(1-|x|),(1-|x|)]$. Here $2 \mu_{B} H(0)=0$ and $2 \mu_{B} H_{c}= \pm \lim _{m \rightarrow \pm[1-|x|]} 2 \mu_{B} H(m)$. A closed-form expression for the dependence on $U, t$, and density $n_{e}$ of the energy scale $2 \mu_{B} H_{c}$ where $H_{c}$ is the critical magnetic field for the onset of fully polarized ferromagnetism is given below.

The intrinsic energies $\varepsilon_{\eta, \pm 1 / 2}$ and $\varepsilon_{s, \pm 1 / 2}$ relative to the zero-energy ground-state level of a unpaired $\eta$-spin $1 / 2$ and a unpaired spin $1 / 2$, respectively, of projection $\pm 1 / 2$ are directly related to the energy scales $2 \mu$ and $2 \mu_{B} H$, respectively. Such intrinsic energies are useful reference scales for the analysis presented below in Section 6.3 of the anti-binding or binding character of the paired spins $1 / 2$ and paired $\eta$-spins $1 / 2$ configuration within each pair. Straightforward calculations relying on the algebra of the $\eta$-spin and spin $S U(2)$ symmetry off-diagonal generators lead to,

$$
\begin{aligned}
\varepsilon_{\eta, \pm 1 / 2} & =2|\mu| \text { and } \varepsilon_{\eta, \mp 1 / 2}=0 \text { for } \operatorname{sgn}\left\{\left(1-n_{e}\right)\right\} 1=\mp 1 \text { and } n_{e} \neq 1, \\
& =\left(\mu^{0} \pm \mu\right) \text { for } n_{e}=1 \text { and } \mu \in\left[-\mu^{0}, \mu^{0}\right], \\
\varepsilon_{s, \pm 1 / 2} & =2 \mu_{B}|H| \text { and } \varepsilon_{s, \mp 1 / 2}=0 \text { for } \operatorname{sgn}\{m\} 1=\mp 1 .
\end{aligned}
$$

Hence a $S_{\alpha}=1 ; S_{\alpha}^{z}=0 \alpha$-multiplet configuration of two unpaired $\eta$-spins or two unpaired spins has an intrinsic energy given by,

$$
\varepsilon_{\alpha+1 / 2}+\varepsilon_{\alpha,-1 / 2}=2 \mu_{\alpha} \text { where } \alpha=\eta, s .
$$



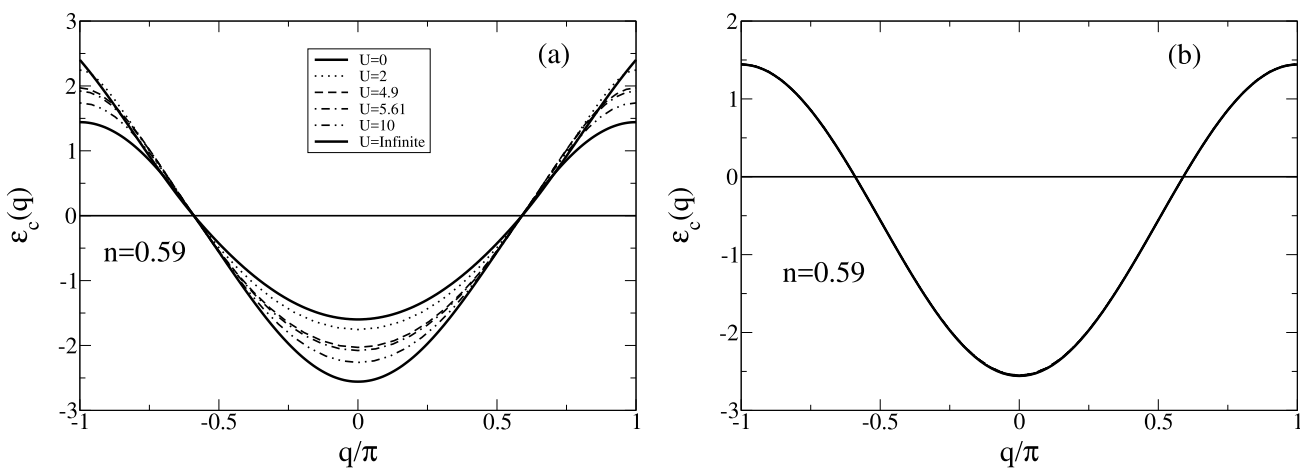

Fig. 10. The $c$ band energy dispersion $\varepsilon_{c}(q)$, Eq. (152) for $\beta=c$, plotted as a function of the momentum in units of $t$ for a set of $U / t$ values (in units of $t$ ), electronic density $n_{e}=0.59$, and spin density (a) $m=0$ and (b) $m \rightarrow n_{e}=0.59$. (As in Ref. [104], in the figures the electronic density $n_{e}$ is denoted by $n$.) Source: The figures plots were produced using the same data as in Fig. 6 of Ref. [104] for other densities.
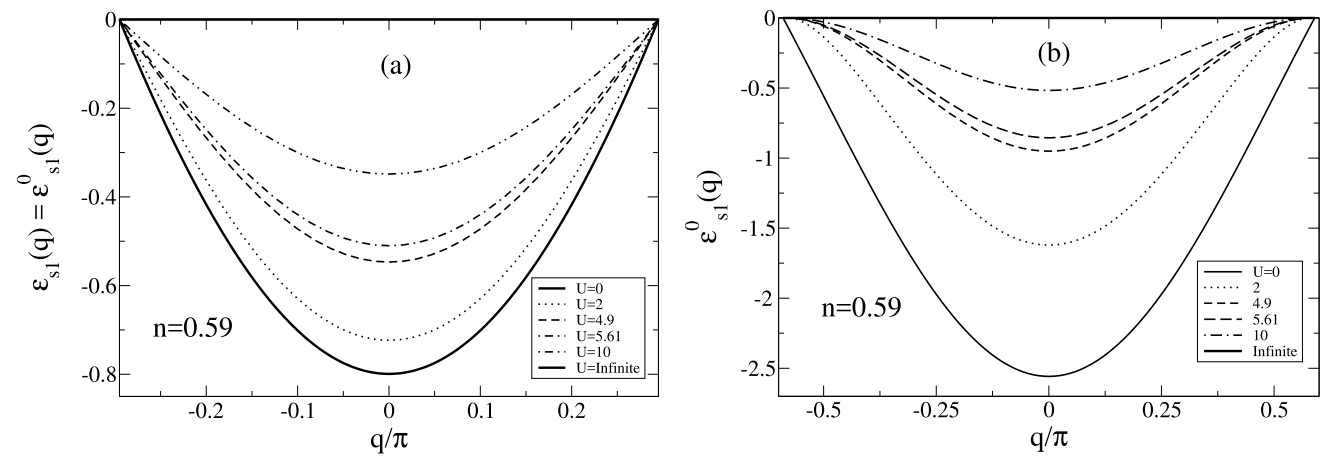

Fig. 11. The $s 1$ band energy dispersion $\varepsilon_{s 1}^{0}(q)$, Eq. (158) for $\alpha n=s 1$, which for $m=0$ equals the related $s 1$ band energy dispersion $\varepsilon_{s 1}(q)$, Eqs. (152) for $\beta=s 1$, plotted as a function of the momentum in units of $t$ for the same $U / t$ and $n_{e}$ values as Fig. 10 and (a) $m=0$ and (b) $m \rightarrow n_{e}=0.59$. (As in Ref. [104], in the figures the electronic density $n_{e}$ is denoted by $n$.)

Source: The figures plots were produced using the same data as in Fig. 7 of Ref. [104] for other densities.

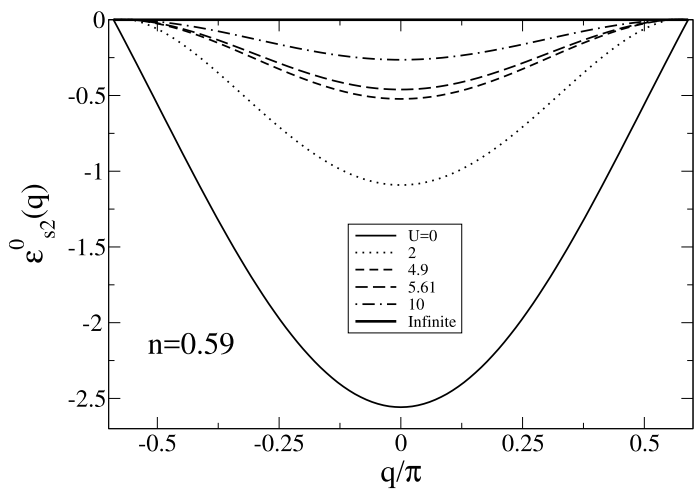

Fig. 12. The $s 2$ band energy dispersion $\varepsilon_{s 2}^{0}(q)$, Eq. (158) for $\beta=s 2$, plotted as a function of the momentum in units of $t$ for the same $U / t$ and $n_{e}$ values as Fig. 10 and $m \rightarrow n_{e}=0.59$. (As in Ref. [104], in the figure the electronic density $n_{e}$ is denoted by $n$.)

Source: The figure plots were produced using data from Ref. [104].

The energy scales $2 \mu_{\alpha}$ such that $2 \mu_{\eta}=2|\mu|$ and $2 \mu_{s}=2 \mu_{B}|H|$ and the Mott-Hubbard gap $2 \mu^{0}$ associated with the energy scale $\mu^{0}$ are those considered above. They are given in Eqs. (B.6) and (B.8) of Appendix B.

The energy dispersions $\varepsilon_{c}(q), \varepsilon_{s 1}^{0}(q), \varepsilon_{s 2}^{0}(q), \varepsilon_{\eta 1}^{0}(q)$, and $\varepsilon_{\eta 2}^{0}(q)$ are plotted as a function of the momentum $q$ in Figs. 10-14, respectively, for several $U / t$ values, electronic density $n_{e}=0.59$, and spin densities $m=0$ and/or $m \rightarrow n_{e}=0.59$. (The electronic density $n_{e}=0.59$ is that used in Refs. $[13,15,16,58]$ for the stacks of TCNQ molecules in TTF-TCNQ.) Analysis of the figures energy-dispersions slopes reveals that the velocity $v_{\beta}(q)$, Eq. (155), vanishes at $q=0$ for all $\beta=c, \alpha n$ branches. 

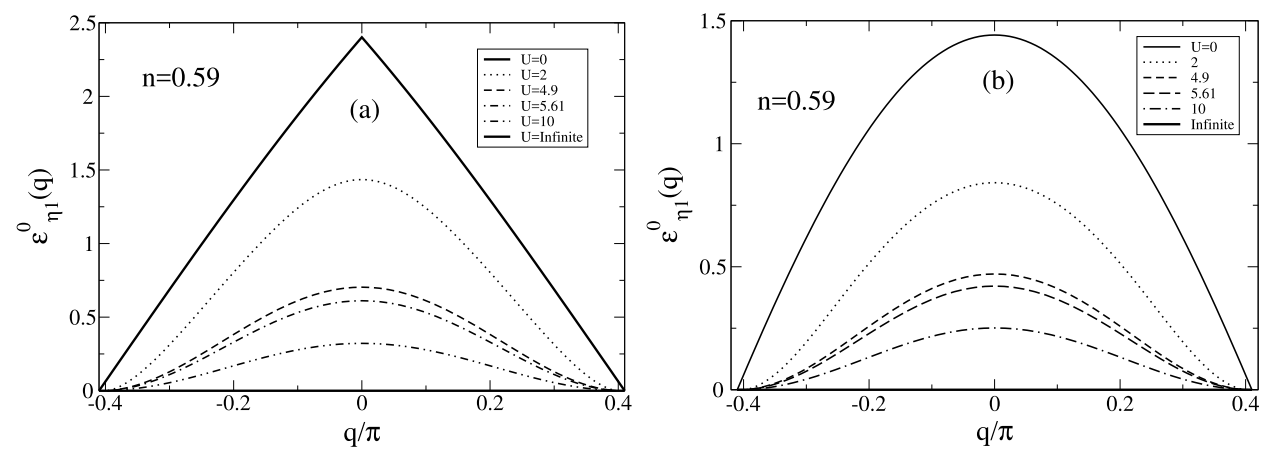

Fig. 13. The $\eta 1$ band energy dispersion $\varepsilon_{\eta 1}^{0}\left(q_{j}\right)$, Eq. (158) for $\beta=\eta 1$, plotted in units of $t$ for the same $U / t$ and $n_{e}$ values as Fig. 10 and (a) $m=0$ and (b) $m \rightarrow n_{e}=0.59$. (As in Ref. [104], in the figures the electronic density $n_{e}$ is denoted by $n$.)

Source: The figures plots were produced using the same data as in Figs. 8(a) and 9(a) of Ref. [104] for other densities.
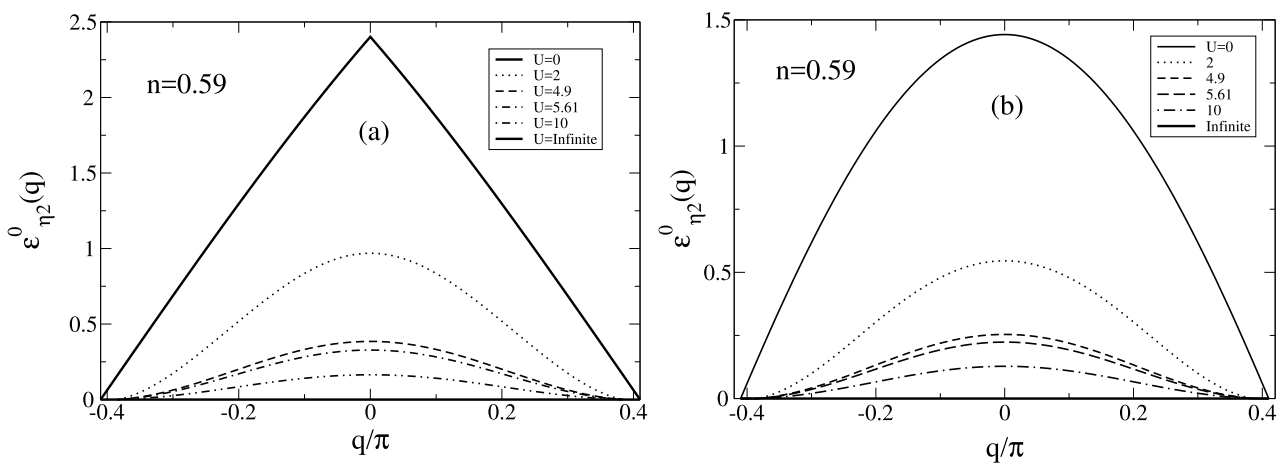

Fig. 14. The $\eta 2$ band energy dispersion $\varepsilon_{\eta 2}^{0}\left(q_{j}\right)$, Eq. (158) for $\beta=\eta 2$, plotted in units of $t$ for the same $U$ and $n$ values (denoted in the figures by $n$ ) as Fig. 10 and (a) $m=0$ and (b) $m \rightarrow n_{e}=0.59$. (As in Ref. [104], in the figures the electronic density $n_{e}$ is denoted by $n$.)

Source: The figures plots were produced using the same data as in Figs. 8(b) and 9(b) of Ref. [104] for other densities.

Provided that $u>0$, it also vanishes at the limiting momentum values $q= \pm q_{\beta}$ for the $\beta \neq s 1$ branches. The energy bandwidths of the $s 1$ band, $s 2$ band, $\eta 1$ band, and $\eta 2$ band, plotted in Figs. 11-14, respectively, vanish in the $u \rightarrow \infty$ limit. (In the $m \rightarrow 0$ limit, the momentum and energy bandwidths of the $s 2$ band energy dispersion vanish for all $u$ values, so that it is not plotted in Fig. 12 for $m=0$.) This $u \rightarrow 0$ behavior results from the degenerescence of all spin configurations and of all $\eta$-spin configurations with the same electron double occupancy reached in that limit. For the $u \rightarrow 0$ and $u \gg 1$ limiting behaviors of the $\beta$ energy dispersions, Eqs. (152) and (158), see Ref. [104].

\subsection{The $c$ and $\alpha$ n pseudoparticle quantum liquid II: Applications to the low-energy physics}

The 1D Hubbard model in general PSs is a quantum liquid of $c$ pseudoparticles and $n=1, \ldots, \infty$ branches of composite $\eta n$-pseudoparticles and $s n$-pseudoparticles. In the following we consider again that model in its metallic-phase PSs whose ground states are LWSs with densities $n_{e} \in\left[0,1\left[\right.\right.$ and $m \in\left[0, n_{e}\right]$ for which $\left(\mu-\mu^{0}\right)>0$ and $H>0$. In the case of that quantum problem, there emerge gaps $\Delta_{\eta}$ and $\Delta_{s}$ between such ground states and their PS excited energy eigenstates populated by $\eta n$ pseudoparticles and $n>1 s n$ pseudoparticles, respectively. Such gaps minimum values are $\Delta_{\eta}^{\min }=\varepsilon_{\eta 1}\left(q_{\eta 1}^{ \pm}\right)=2|\mu|$ and $\Delta_{s}^{\min }=\varepsilon_{s 2}(0)=4 \mu_{B}|H|-\left|\varepsilon_{s 2}^{0}(0)\right|$. For excitation energy below these gaps, the physics is that of two $U(1)$ symmetry quantum problems. The corresponding energy and momentum eigenstates are described by only groups of real rapidities. (This is as in the case of the 1D Lieb-Liniger Bose gas.) The $\eta 1$ pseudoparticle energy spectrum is gapped, in spite of corresponding to real rapidities. This results from creation of one $\eta 1$ pseudoparticle involving creation of one rotated-electron doubly occupied site. (In the attractive $U<01 \mathrm{D}$ Hubbard model the situation is the opposite, with the $s 1$ pseudoparticle energy spectrum gaining a gap and the $\eta 1$ pseudoparticle spectrum being gapless.)

In the present case of chemical-potential values $\left(\mu-\mu^{0}\right)>0$ and magnetic fields $H>0$, the model static and lowtemperature properties are determined by excitations associated with energy and momentum eigenstates with finite $c$ and $s 1$ pseudoparticle occupancy $N_{c}=N_{e}$ and $N_{s 1}=N_{e \downarrow}=\left(N_{e}-2 S_{s}\right) / 2$ only in the $c$ and $s 1$ bands, respectively. This applies as well to the finite-energy dynamical correlation functions leading order contributions. Hence for such states $N_{\eta n}=0$ for all $n=1, \ldots, \infty$ and $N_{s n}=0$ for $n>1$. On the one hand, due to the $n_{e}=1$ Mott-Hubbard gap, the physics of the 
model Mott-Hubbard insulator phase is qualitatively different from that of its $n_{e} \neq 1$ metallic phase. On the other hand, the physical quantities have the same values both at $H=0$ and in the $H \rightarrow 0$ limit, respectively.

For the quantum problem under consideration here, the following parameters involving the dressed phases shift $2 \pi \Phi_{\beta \beta^{\prime}}\left(q_{j}, q_{j^{\prime}}\right)$, Eq. (154), in units of $2 \pi$ and with the two momentum values $q_{j}$ and $q_{j^{\prime}}$ at the $\beta, \beta^{\prime}=c, s 1$ Fermi points play an important role in the static and low-temperature properties [89,90,92,93],

$$
\xi_{\beta \beta^{\prime}}^{j}=\delta_{\beta, \beta^{\prime}}+\sum_{\iota= \pm}(\iota)^{j} \Phi_{\beta \beta^{\prime}}\left(q_{F \beta}, \iota q_{F \beta^{\prime}}\right) \text { where } \beta, \beta^{\prime}=c, s 1 \text { and } j=0,1 .
$$

(For $\beta=\beta^{\prime}$ and $\iota=1$ in Eq. (162), the present TL notation assumes that the two $\beta=c, s 1$ Fermi momenta in the argument of $\Phi_{\beta, \beta}\left(q_{F \beta}, q_{F \beta}\right)$, differ by $2 \pi / L$, whereas that phase shift vanishes for identical momentum values.)

The dressed phase-shift related anti-symmetrical parameters $\xi_{\beta \beta^{\prime}}^{1}$ and symmetrical parameters $\xi_{\beta \beta^{\prime}}^{0}$ turn out to be the entries of the conformal-field theory $2 \times 2$ dressed-charge matrix and of the transposition of its inverse matrix $[90,92,176,181,224]$,

$$
Z^{1}=\left[\begin{array}{ll}
\xi_{c c}^{1} & \xi_{c s 1}^{1} \\
\xi_{s 1 c}^{1} & \xi_{s 1 s 1}^{1}
\end{array}\right] ; \quad Z^{0}=\left[\begin{array}{ll}
\xi_{c c}^{0} & \xi_{c s 1}^{0} \\
\xi_{s 1 c}^{0} & \xi_{s 1 s 1}^{0}
\end{array}\right] ; \quad \lim _{m \rightarrow 0} Z^{1}=\left[\begin{array}{cc}
\xi_{0} & \xi_{0} / 2 \\
0 & 1 / \sqrt{2}
\end{array}\right] ; \quad \lim _{m \rightarrow 0} Z^{0}=\left[\begin{array}{cc}
1 / \xi_{0} & 0 \\
-1 / \sqrt{2} & \sqrt{2}
\end{array}\right],
$$

respectively, where $Z^{0}=\left(\left(Z^{1}\right)^{-1}\right)^{T}$. (The dressed-charge matrix definition of Ref. [176] has been used here, which is the transposition of that of Ref. [224].) The $m \rightarrow 0$ phase-shift parameter $\xi_{0}$ in Eq. (163) is given by $\xi_{0}=\xi_{0}\left(r_{c}^{0}\right)$. The function $\xi_{0}(r)$ is the unique solution of the integral equation, Eq. (74) of Ref [92] for $x=r$. That parameter has limiting values $\xi_{0}=\sqrt{2}$ for $u \rightarrow 0$ and $\xi_{0}=1$ for $u \rightarrow \infty$.

At low energy the present quantum problem can be described by a two-component TLL [2-4,44,47,48]. The corresponding $g$ matrix [4] can be expressed in terms of the dressed phase-shift parameters, Eq. (162). A low-energy physical quantity that is fully controlled by the above phase-shift related parameters in Eq. (163) is the exponent in the low-energy $\omega$ power-law dependence of the electronic density of states suppression, $\propto|\omega|^{\alpha_{0}}$. In the $m \rightarrow 0$ limit its following expression involves only the parameter $\xi_{0}$ in that equation,

$$
\alpha_{0}=\frac{\left(2-\xi_{0}^{2}\right)^{2}}{8 \xi_{0}^{2}} \in[0,1 / 8] .
$$

The exponent, Eq. (164), has limiting values $\alpha_{0}=0$ for $u \rightarrow 0$ and $\alpha_{0}=1 / 8$ for $u \rightarrow \infty$, respectively.

By use of the methods reported for the 1D Lieb-Liniger Bose gas and spin-1/2 XXX chain, one finds that for (i) electronic densities $n_{e}$ and (ii) spin densities $m$ not too near (i) 0 and 1 (ii) and $n_{e}$, respectively, the low-temperature specific heat reads [89],

$$
\frac{c_{V}}{L}=\frac{k_{B} \pi}{3}\left(\frac{1}{v_{c}}+\frac{1}{v_{s 1}}\right)\left(k_{B} T\right),
$$

where $v_{c}$ and $v_{s 1}$ are the $\beta=c, s 1$ band Fermi velocities in Eq. (155).

On the one hand, for electronic densities $n_{e} \in 10,1$ [ not too close to $n_{e}=0$ and $n_{e}=1$ the low-temperature thermal excitations that contribute to the first term of the specific heat expression, Eq. (165), refer to a well-defined branch of gapless excited energy and momentum eigenstates. Their charge degrees of freedom are generated from the ground state by lowenergy and small-momentum particle-hole processes around the $c$ band Fermi points.

On the other hand, as for the spin-1/2 XXX chain, at zero magnetic field $H=0$ the spin degrees of freedom of such gapless branch of states that contribute to the second term of the specific heat expression, Eq. (165), refer to sn-strings of lengths $n>1$. For finite magnetic field these $n>1 s n$-strings excitations become gapped. The thermal excitations that contribute to the low-temperature specific heat become gapless excited energy and momentum eigenstates whose charge degrees of freedom remain being generated by low-energy and small-momentum particle-hole processes around the $c$ band Fermi points. Their spin degrees of freedom correspond to spin real rapidities. Those are generated from the ground state by low-energy and small-momentum particle-hole processes around the $s 1$ band Fermi points.

The specific-heat expression obtained for $H>0$ leads in the $H \rightarrow 0$ limit to the correct $H=0$ expression. On the contrary, the specific heat expression, Eq. (165), is not valid in the $m \rightarrow n_{e}$ limit. This is because it does not describe properly the crossover to the specific heat exponential regime. The latter arises due to the gap $2 \mu_{B}\left(H-H_{C}\right)$ in the excitation spectrum for $H>H_{c}$. More generally, the validity of that specific heat expression refers to very low temperatures $T \ll 2 \mu_{B}\left(H_{c}-H\right) / k_{B}$ for $\left(H_{c}-H\right)>0, T \ll 2\left(\mu-\mu_{0}\right) / k_{B}$ for $\left(\mu-\mu_{0}\right)>0$, and $T \ll 2\left(\mu_{1}-\mu\right) / k_{B}$ for $\left(\mu_{1}-\mu\right)>0$. In the close neighborhood of $\mu=\mu_{1}=U / 2+2 t$, the problem is trivial. The specific heat is then given by its noninteracting value. Following the technical similarities of the crossover critical regimes associated with the $2 \mu_{B}\left|H-H_{c}\right| \ll k_{B} T$ and $2\left|\mu-\mu_{0}\right| \ll k_{B} T$ limits [89], here we shortly discuss the former regime for electronic densities $\left.n_{e} \in\right] 0,1\left[\right.$ not too close to $n_{e}=0$ and $n_{e}=1$.

Near $H=H_{c}$ the minimum gap for energy eigenstates with spin sn-strings of length $n>1$ associated with complex rapidities is given by,

$$
\Delta_{s}^{\min }=4 \mu_{B} H_{c}-W_{s 2} .
$$


The dependence on $U, t$, and $n_{e}$ of the energy scale $4 \mu_{B} H_{c}$ appearing here is given in the following. $W_{s 2} \equiv\left|\varepsilon_{s 2}^{0}(0)\right|$ is in Eq. (166) the energy scale in Eq. (B.10) of Appendix B for $n=2$. Its limiting behaviors for $u \rightarrow 0$ and $u \gg 1$ are,

$$
\begin{aligned}
\Delta_{s}^{\min } & =2 \mu_{B} H_{c}=4 t \sin ^{2}\left(\frac{\pi n_{e}}{2}\right) \text { for } u \rightarrow 0 \\
& =3 \mu_{B} H_{c}=\frac{12 n_{e} t^{2}}{U}\left(1-\frac{\sin \left(2 \pi n_{e}\right)}{2 \pi n_{e}}\right) \text { for } u \gg 1,
\end{aligned}
$$

respectively.

We consider low temperatures $T<\Delta_{s}^{\min } / k_{B}$ within the critical regime of the crossover to ferromagnetism for which $2 \mu_{B}\left|H-H_{c}\right| \ll k_{B} T$. For such temperatures the energy and momentum eigenstates that contribute to the specific heat have spin degrees of freedom described only by spin sn-strings of length $n=1$. They are thus only populated by $s 1$ pseudoparticles and unpaired spins $1 / 2$. At low temperatures the crossover regime involves both the above $s 1$ band gapless spin-singlet excited states and across-gap excited energy eigenstates. The latter states spin degrees of freedom are generated by elementary spin-triplet $\delta S_{s}= \pm 1$ processes. (They are similar to those considered for the spin- $1 / 2 X X X$ chain in Section 3.6.)

The $s 1$ band energy dispersion valid for spin density $m \rightarrow n_{e}$ reads [89],

$$
\left.\varepsilon_{s 1}\left(q_{j}\right) \approx \frac{q_{j}^{2}}{2 m_{s 1}^{*}}-2 \mu_{B}\left(H_{c}-H\right) \text { for } n_{e} \in\right] 0,1\left[\text { and } m \rightarrow n_{e} .\right.
$$

Here the critical magnetic energy $2 \mu_{B} H_{c}$ is that associated with the zero-temperature critical magnetic field $H_{c}$ for the onset of fully polarized ferromagnetism. That energy scale and the effective spin-triplet mass $m_{s 1}^{*}$ in Eq. (168) are given by [89],

$$
\begin{aligned}
2 \mu_{B} H_{c}= & \sqrt{(4 t)^{2}+U^{2}} \frac{1}{\pi} \arctan \left(\frac{\sqrt{(4 t)^{2}+U^{2}}}{U} \tan \left(\pi n_{e}\right)\right)-U n_{e}-4 t \cos \left(\pi n_{e}\right) \frac{1}{\pi} \arctan \left(\frac{4 t \sin \left(\pi n_{e}\right)}{U}\right) \\
& \text { for } \left.n_{e} \in\right] 0,1\left[\text { and } m \rightarrow n_{e},\right.
\end{aligned}
$$

and

$$
\left.m_{s 1}^{*}=\frac{U}{4 t^{2}} \frac{\frac{\sqrt{(4 t)^{2}+U^{2}}}{U} \frac{1}{\pi} \arctan \left(\frac{\sqrt{(4 t)^{2}+U^{2}}}{U} \tan \left(\pi n_{e}\right)\right)}{1-\frac{\sqrt{(4 t)^{2}+U^{2}}}{U} \frac{1}{1+\left(\frac{4 t \sin \left(\pi n_{e}\right)}{U}\right)^{2}} \frac{\sin \left(2 \pi n_{e}\right)}{2 \arctan \left(\frac{\sqrt{(4 t)^{2}+U^{2}}}{U} \tan \left(\pi n_{e}\right)\right)}} \text { for } n_{e} \in\right] 0,1\left[\text { and } m \rightarrow n_{e},\right.
$$

respectively. Their limiting behaviors for $u \rightarrow 0$ and $u \gg 1$ read,

$$
\begin{aligned}
& m_{s 1}^{*}=\frac{1}{2 t} \text { and } 2 \mu_{B} H_{c}=4 t \sin ^{2}\left(\frac{\pi n_{e}}{2}\right) \text { for } u \rightarrow 0 \\
& m_{s 1}^{*}=\frac{U}{4 t^{2}} \frac{n_{e}}{\left(1-\frac{\sin \left(2 \pi n_{e}\right)}{2 \pi n_{e}}\right)} \text { and } 2 \mu_{B} H_{c}=\frac{8 n_{e} t^{2}}{U}\left(1-\frac{\sin \left(2 \pi n_{e}\right)}{2 \pi n_{e}}\right) \text { for } u \gg 1,
\end{aligned}
$$

respectively. The critical magnetic field $H_{c}$ in Eq. (169) and the inverse effective spin-triplet mass $1 / m_{s 1}^{*}$, Eq. (170), are plotted in Fig. 15 as a function of the electronic density $n_{e}$ and of $U / t$ for various values of $U / t$ and $n_{e}$, respectively.

For the crossover critical regime under consideration, the scaling function of the specific heat is found to be given by,

$$
\begin{gathered}
\frac{c_{V}}{L}=\frac{k_{B} \pi}{3 v_{c}} k_{B} T+\sqrt{\frac{2 m_{s 1}^{*} k_{B} T}{\pi}}\left(-\frac{3}{8} f_{3 / 2}^{s}+\frac{1}{2}\left(\frac{2 \mu_{B}\left(H_{c}-H\right)}{k_{B} T}\right) f_{1 / 2}^{s}-\frac{1}{2}\left(\frac{2 \mu_{B}\left(H_{c}-H\right)}{k_{B} T}\right)^{2} f_{-1 / 2}^{s}\right), \\
\text { where } v_{c}=2 t \sin \left(\pi n_{e}\right), \quad f_{l}^{s}=\operatorname{Li}_{l}\left(-e^{\frac{2 \mu_{B}\left(H_{c}-H\right)}{k_{B} T}}\right) \text { and } \operatorname{Li}_{l}(x)=\sum_{j=1}^{\infty} \frac{x^{j}}{j^{l}} .
\end{gathered}
$$

Expanding this scaling function up to first order in $2 \mu_{B}\left(H_{c}-H\right) / k_{B} T$, one finds that the low-temperature specific heat behaves in a small field window $2 \mu_{B}\left|H-H_{c}\right| \ll k_{B} T$ around $H_{c}$ as [89],

$$
\frac{c_{V}}{L}=\frac{k_{B} \pi}{3 v_{c}} k_{B} T+k_{B} c_{0} \sqrt{\frac{m_{s 1}^{*} k_{B} T}{2}}\left(c_{1}+c_{2} \frac{2 \mu_{B}\left(H_{c}-H\right)}{k_{B} T}\right) \text { where } 2 \mu_{B}\left|H-H_{c}\right| \ll k_{B} T .
$$

The term emerging here from the spin degrees of freedom has the same form as that provided in Eq. (89) and the coefficients $c_{0}, c_{1}$, and $c_{2}$ are thus also those given in Eq. (90). (Also the calculations to reach Eq. (173) are similar to those presented in Appendix D for the corresponding specific heat scaling function of the spin-1/2 XXX chain [206].) 

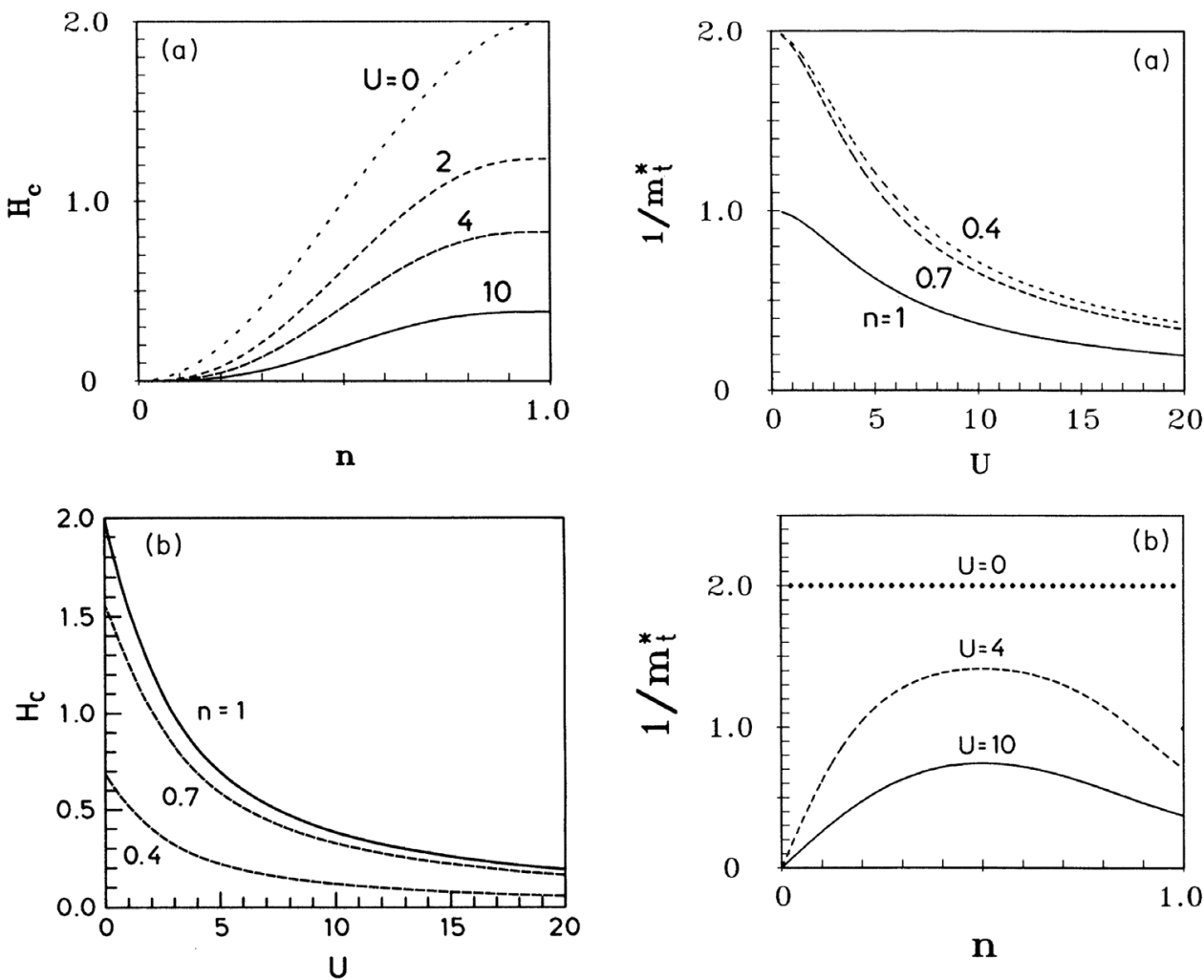

Fig. 15. The critical magnetic field $H_{c}$ for the onset of fully polarized ferromagnetism in Eq. (169) and the inverse effective spin-triplet mass $1 / m_{s 1}^{*}$, Eq. (170), versus the electronic density $n_{e}$ for $U / t=0, U / t=2$, and $U / t=10$ and versus $U / t$ for $n_{e}=0.4, n_{e}=0.7$, and $n_{e}=1.0$. Notice that at $n_{e}=1,1 / m_{s 1}^{*} \rightarrow t$ as $U / t \rightarrow 0$. This singular behavior is due to the Mott-Hubbard insulator transition. (As in Ref. [89], in the figures the electronic density $n_{e}$ and effective spin-triplet mass $m_{s 1}^{*}$ are denoted by $n$ and $m_{t}^{*}$, respectively.)

Source: From Ref. [89].

In the crossover critical regime defined by Eq. (173) both the gapless and across-gap channels associated with spin-singlet excitations and excitations generated by elementary spin-triplet processes, respectively, are thermally active. That equation is only valid for a very narrow region around $H_{c}$.

The charge response function or compressibility $\alpha=\eta$ and spin response function $\alpha=s$,

$$
\left.\chi_{\eta}\right|_{y}=-\frac{1}{n_{e}^{2}} \frac{1}{\partial \mu\left(n_{e}\right) /\left.\partial n_{e}\right|_{y}} \text { where } y=H, m \text { and }\left.\chi_{s}\right|_{z}=-\frac{2 \mu_{B}}{\partial H(m) /\left.\partial m\right|_{z}} \text { where } z=\mu, n_{e},
$$

are controlled by the dressed phase-shift parameters, Eq. (162). Such functions involve partial derivatives of the chemical potential and magnetic field, Eq. (159).

By use of techniques similar to those of a Fermi liquid, which account for the $\beta=c, s 1$ and $\beta^{\prime}=c, s 1$ pseudoparticle zero-momentum forward-scattering interactions associated with the $f$ functions, Eq. (156), the studies of Ref. [92] have found,

$$
\begin{aligned}
& \left.\chi_{\eta}\right|_{H}=\frac{1}{\pi n_{e}^{2}} \sum_{\beta=c, s 1} \frac{\left(\xi_{\beta c}^{1}\right)^{2}}{v_{\beta}} \text { and }\left.\chi_{\eta}\right|_{m}=\frac{1}{\pi n_{e}^{2}} \frac{1}{\sum_{\beta=c, s 1} v_{\beta}\left(\xi_{\beta c}^{0}+\xi_{\beta s 1}^{0} / 2\right)^{2}}, \\
& \left.\chi_{s}\right|_{\mu}=\frac{\mu_{B}^{2}}{\pi} \sum_{\beta=c, s 1} \frac{\left(\xi_{\beta c}^{1}-2 \xi_{\beta s 1}^{1}\right)^{2}}{v_{\beta}} \text { and }\left.\chi_{s}\right|_{n_{e}}=\frac{\mu_{B}^{2}}{\pi} \frac{1}{\sum_{\beta=c, s 1} v_{\beta}\left(\xi_{\beta s 1}^{0} / 2\right)^{2}} .
\end{aligned}
$$

The derivation in Ref. [92] of the charge and spin response functions, Eq. (174), expressions provided in Eq. (175) uses the general procedures reported in Appendix $\mathrm{E}$ to obtain the corresponding expression of the spin response function of the spin-1/2 XXX chain. Such two-electron response functions expressions can be understood as being controlled by parameters that play the same role as the Landau parameters in a Fermi liquid. For the present $c$ and $s 1$ pseudoparticle quantum liquid, 
the Landau parameters can have a $i=0$ symmetrical or $i=1$ anti-symmetrical character. They read,

$$
\begin{aligned}
g_{\beta \beta}^{i} & =1+\frac{1}{2 \pi v_{\beta}} \sum_{\iota= \pm 1}(\iota)^{i} f_{\beta \beta}\left(q_{F \beta}, \iota q_{F \beta}\right) \\
& =\frac{1}{v_{\beta}}\left(v_{c}\left(\xi_{c \beta}^{i}\right)^{2}+v_{s 1}\left(\xi_{s 1 \beta}^{i}\right)^{2}\right) \text { for } \beta=c, s 1 \text { and } i=0,1, \\
g_{\beta \beta^{\prime}}^{i} & =\frac{1}{2 \pi v_{\beta}} \sum_{\iota= \pm 1}(\iota)^{i} f_{\beta \beta^{\prime}}\left(q_{F \beta}, \iota q_{F \beta^{\prime}}\right) \\
& =\frac{1}{v_{\beta}}\left(v_{c} \xi_{c c}^{i} \xi_{c s 1}^{i}+v_{s 1} \xi_{s 1 s 1}^{i} \xi_{s 1 c}^{i}\right) \text { for } \beta \neq \beta^{\prime}=c, s 1 \text { and } i=0,1 .
\end{aligned}
$$

Such parameters expressions contain the $c$ and $s 1$ bands velocities at a Fermi point defined in Eq. (155). They also contain two $f$ functions, Eq. (156), with the two momenta at Fermi points pointing in the same and in opposite directions, respectively. The $i=0$ symmetrical and $i=1$ anti-symmetrical pseudoparticle Landau parameters involve the sum and difference of these two $f$ functions, respectively.

As given in Eq. (176), the pseudoparticle Landau parameters can be expressed in terms of the $\beta=c, s 1$ velocities at a Fermi point and of the dressed phase-shift related $i=1$ anti-symmetrical and $i=0$ symmetrical parameters $\xi_{\beta \beta^{\prime}}^{i}$. Those are the entries of the conformal-field theory dressed charge matrix $Z^{1}$ and of the matrix $Z^{0}=\left(\left(Z^{1}\right)^{-1}\right)^{T}$, respectively, Eq. (163). Such entries naturally emerge within the expressions of the $i=0,1$ and $\beta=c, s 1$ renormalized velocities $v_{\beta \beta}^{i} \equiv v_{\beta} g_{\beta \beta}^{i}=v_{\beta}+\frac{1}{2 \pi} \sum_{\iota= \pm 1}(\iota)^{i} f_{\beta \beta}\left(q_{F \beta}, \iota q_{F \beta}\right)$ and $v_{\beta \beta^{\prime}}^{i} \equiv v_{\beta} g_{\beta \beta^{\prime}}^{i}=\frac{1}{2 \pi} \sum_{l= \pm 1}(\iota)^{i} f_{\beta \beta^{\prime}}\left(q_{F \beta}, \iota q_{F \beta^{\prime}}\right)$ where $\beta \neq \beta^{\prime}$. The pseudoparticle representation goes though beyond conformal-field theory. Indeed, the group velocities and $f$ functions in these expressions are also well defined for arbitrary $\beta$-band momentum values, $v_{\beta}\left(q_{j}\right)$ and $f_{\beta \beta^{\prime}}\left(q_{j}, q_{j^{\prime}}\right)$ for both $\beta=\beta^{\prime}$ and $\beta \neq \beta^{\prime}$. Beyond that theory, they also exist within the pseudoparticle representation for all $c, \eta n$, and sn branches where $n=1, \ldots, \infty$.

The charge and spin response functions expressions, Eqs. (174) and (175), can be expressed in terms of the Fermi-point $c$ and $s 1$ group velocities and the $i=0$ symmetrical pseudoparticle Landau parameters as follows,

$$
\begin{aligned}
& \left.\chi_{\eta}\right|_{H}=\frac{1}{\pi n_{e}^{2} v_{c}} \frac{1}{\left(g_{c c}^{0}-\frac{g_{c s}^{0} g_{s 1 c}^{0}}{g_{s 1 s 1}^{0}}\right)} \text { and }\left.\chi_{\eta}\right|_{m}=\frac{1}{\pi n_{e}^{2} v_{c}} \frac{1}{\left(g_{c c}^{0}+\frac{v_{s 1}}{4 v_{c}} g_{s 1 s 1}^{0}+g_{c s 1}^{0}\right)}, \\
& \left.\chi_{s}\right|_{\mu}=\frac{\mu_{B}^{2}}{\pi v_{s 1}} \frac{\left(g_{s 1 s 1}^{0}+\frac{4 v_{c}}{v_{s 1}} g_{c c}^{0}+4 g_{s 1 c}^{0}\right)}{\left(\frac{v_{c}}{v_{s 1}} g_{s 1 s 1}^{0} g_{c c}^{0}-\left(g_{s 1 c}^{0}\right)^{2}\right)} \text { and }\left.\chi_{s}\right|_{n_{e}}=\frac{4 \mu_{B}^{2}}{\pi v_{s 1}} \frac{1}{g_{s 1 s 1}^{0}} .
\end{aligned}
$$

For two-electron quantities such as the charge and spin response functions, this renormalization is qualitatively similar to that of a Fermi liquid. Indeed, that liquid Landau parameters, which control the effects of the electronic interactions onto the low-energy quantities, are expressed in terms of the $f$ functions associated with the low-energy quasiparticles residual interactions. Similarly, here the residual pseudoparticle interactions associated with the $f$ functions in the parameters expressions, Eq. (176), play exactly the same role.

In the limit of zero magnetic field and thus of zero spin density, the residual pseudoparticle interactions occur only through the parameter $\xi_{0}$. In that limit they read,

$$
\left.\chi_{\eta}\right|_{H}=\left.\chi_{\eta}\right|_{m}=\frac{\xi_{0}^{2}}{\pi n_{e}^{2} v_{c}} \text { and }\left.\chi_{s}\right|_{\mu}=\left.\chi_{s}\right|_{n_{e}}=\frac{2 \mu_{B}^{2}}{\pi v_{s 1}} .
$$

It then follows that $\left.\lim _{u \rightarrow 0} \chi_{\eta}\right|_{H}=2 /\left(\pi n_{e}^{2} v_{c}\right)$ where $v_{c}=2 t \sin \left(\frac{\pi}{2} n_{e}\right)$ and $\left.\lim _{u \rightarrow \infty} \chi_{\eta}\right|_{H}=1 /\left(\pi n_{e}^{2} v_{c}\right)$ where $v_{c}=$ $2 t \sin \left(\pi n_{e}\right)$. Moreover, $\left.\lim _{u \rightarrow \infty} \chi_{s}\right|_{\mu}=\left.\lim _{u \rightarrow \infty} \chi_{s}\right|_{n_{e}} \rightarrow \infty$ because $v_{s 1} \rightarrow 0$ in that limit.

In the spin density $m \rightarrow n_{e}$ limit of the fully polarized ferromagnetism, one finds,

$$
\begin{aligned}
& \left.\chi_{\eta}\right|_{H}=\frac{1}{\pi n_{e}^{2}}\left(\frac{1}{v_{c}}+\frac{\eta_{0}^{2}}{v_{s 1}}\right) \rightarrow \infty \text { and }\left.\chi_{\eta}\right|_{m}=\frac{4}{\pi n_{e}^{2}} \frac{1}{\left(v_{c}\left(2-\eta_{0}\right)^{2}+v_{s 1}\right)} \rightarrow \frac{1}{\pi n_{e}^{2} v_{c}} \frac{1}{\left(1-\eta_{0} / 2\right)^{2}}, \\
& \left.\chi_{s}\right|_{\mu}=\frac{\mu_{B}^{2}}{\pi v_{c}}\left(1+\frac{4 v_{c}}{v_{s 1}}\left(1-\eta_{0} / 2\right)^{2}\right) \rightarrow \infty \text { and }\left.\chi_{s}\right|_{n_{e}}=\frac{4 \mu_{B}^{2}}{\pi} \frac{1}{v_{s 1}+v_{c} \eta_{0}^{2}} \rightarrow \frac{4 \mu_{B}^{2}}{\pi v_{c}} \frac{1}{\eta_{0}^{2}} .
\end{aligned}
$$

Here $\eta_{0}=\frac{2}{\pi} \arctan \left(\frac{\sin \left(\pi n_{e}\right)}{u}\right), v_{c}=2 t \sin \left(\pi n_{e}\right)$, and $v_{s 1} \rightarrow 0$. The inverse of the compressibility $\left.\chi_{\eta}\right|_{H}$ and the inverse of the spin response function $\left.\chi_{s}\right|_{\mu}$, Eqs. (174), (175), and (177), and the spin density $m$ are plotted in Fig. 16 as a function of $U / t$ at electronic density $n_{e}=0.7$ and various values of the magnetic field $H$ in suitable units and as a function of $n_{e}$ for $H=0.1$ and various values of $U / t$.

The 1D Hubbard model quantum phase transitions driven by a change in the chemical potential $\mu$ or the magnetic field $H$, Eq. (159), are marked by the leading divergences of the ground-state onsite entanglement entropy $\mathcal{E}$ derivatives, $\partial \mathcal{E} / \partial \mu$ and 

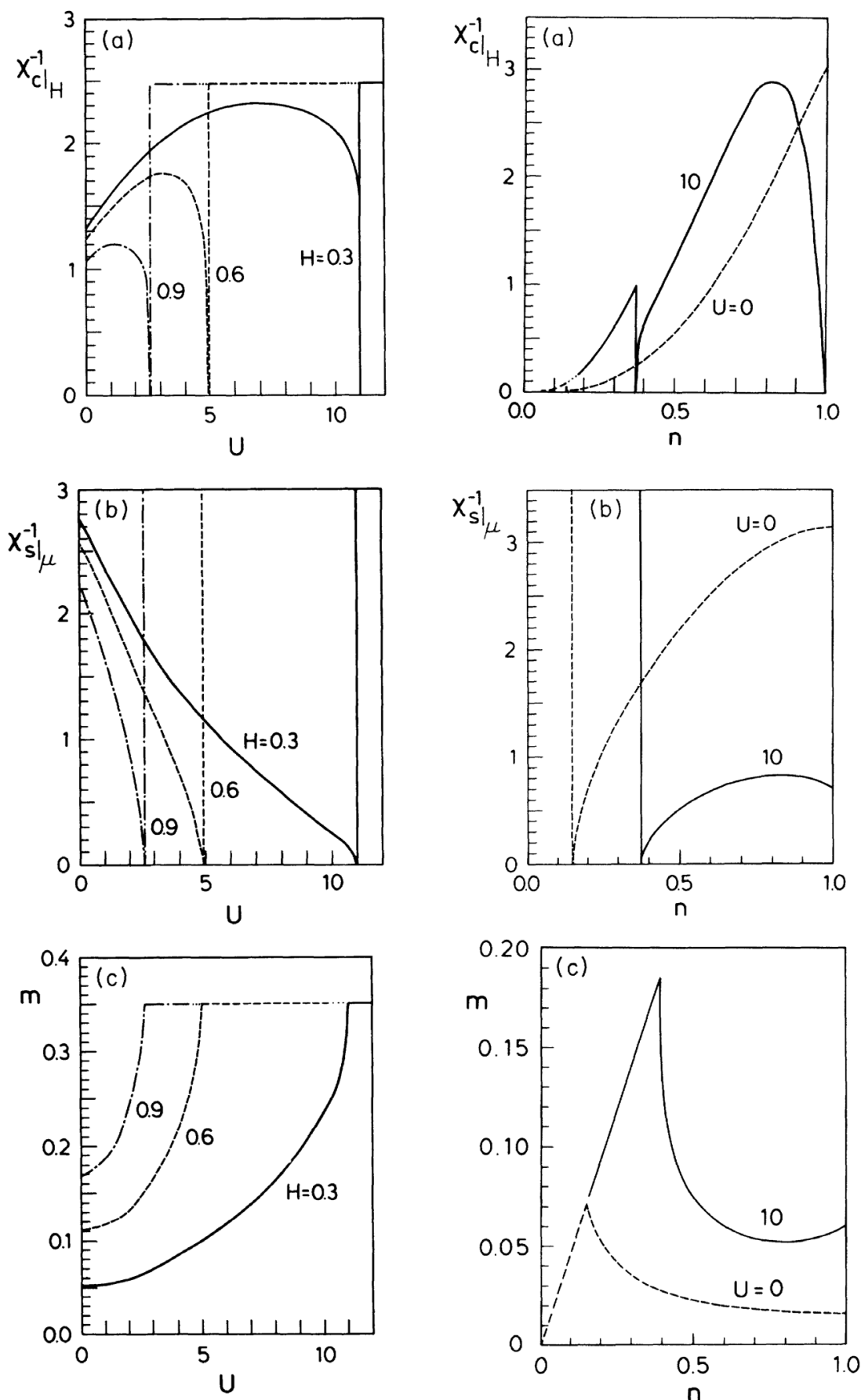

Fig. 16. The inverse of the compressibility $\left.\chi_{\eta}\right|_{H}$ and the inverse of the spin response function $\left.\chi_{s}\right|_{\mu}$, Eqs. (174), (175), and (177), and the spin density $m$ versus $U / t$ at electronic density $n_{e}=0.7$ and values of the magnetic field $H=0.3$ (solid line), $H=0.6$ (dashed line), and $H=0.9$ (dashed-dotted line) in suitable units in the left panels and versus the electronic density $n_{e}$ for $H=0.1$ and values of the on-site repulsion $U / t=0$ (dashed line) and $U / t=10$ (solid line) in the right panels. The discontinuities occur at $U / t$ or $n_{e}$ values at which according to Eq. (169) the magnetic field value $H$ under consideration becomes $H_{c}$ and thus the system becomes ferromagnetic. Note the different behavior at $U / t=0$ and $U / t=10$ of $\left.\chi_{\eta}\right|_{H}$ around $n_{e}=1$ due to the Mott-Hubbard insulator transition. (The spin density $m$ of Ref. [92] is half of that considered in this paper so that in the figures the fully polarized ferromagnetism is reached in the limit of $m \rightarrow n_{e} / 2$, which corresponds to $m \rightarrow n_{e}$ in this review. As in that reference, in the figures the electronic density $n_{e}$ and compressibility $\chi_{\eta}$ are denoted by $n$ and $\chi_{c}$, respectively.) Source: From [92]. 
$\partial \mathcal{E} / \partial H$, respectively [265]. For $n_{e} \neq 1$, they can alternatively be signed by the related derivatives, $\partial \mathcal{E} / \partial n_{e}=-n_{e}^{2} \chi_{\eta} \partial \mathcal{E} / \partial \mu$ and $\partial \mathcal{E} / \partial m=-\left[\chi_{s} / 2 \mu_{B}\right] \partial \mathcal{E} / \partial H[266]$. ( $\chi_{\eta}$ diverges at $n_{e}=1$.)

Also charge $(\alpha=\eta)$ and spin $(\alpha=s)$ stiffnesses $D_{\alpha}$ in the corresponding $\alpha$ conductivity real part $\sigma_{\alpha}(\omega)=2 \pi D_{\alpha} \delta(\omega)+$ $\sigma_{\alpha}^{\text {reg }}(\omega)$ are at zero temperature fully controlled by the dressed phase-shift parameters, Eq. (162). As for the spin-1/2 XXX chain, those are important physical quantities. A finite charge and/or spin stiffness implies the occurrence of charge and/or spin ballistic transport, respectively. Such ballistic transport occurs in 1D correlated even at finite temperatures [103,264]. Recently, a general formalism of hydrodynamics for the 1D Hubbard model was introduced in Ref. [267]. By linearizing hydrodynamic equations, the exact closed-form stiffnesses expressions valid on the hydrodynamic scale are accessed.

For simplicity, here we focus our analysis on the zero-temperature charge and spin stiffnesses. Specifically, relying on charge and spin conservation laws to derive the elementary currents that contribute to such $\alpha=\eta, s$ stiffnesses, the studies of Ref. [93] found that they read,

$$
2 \pi D_{\eta}=j_{c}^{\eta} \text { and } 2 \pi D_{s}=j_{c}^{s}-2 j_{s 1}^{s} .
$$

The elementary currents in these expressions involve the $\beta=c, s 1$ band Fermi velocities, Eq. (155), and $\beta, \beta^{\prime}=c, s 1$ anti-symmetrical dressed phase-shift parameters $\xi_{\beta, \beta^{\prime}}^{1}$, Eq. (162). They are given by,

$$
\begin{aligned}
& j_{\beta}^{\eta}=v_{c} \xi_{c c}^{1} \xi_{c, \beta}^{1}+v_{s 1} \xi_{s 1 c}^{1} \xi_{s 1, \beta}^{1} \text { where } \beta=c, s 1, \\
& j_{\beta}^{s}=v_{c}\left(\xi_{c c}^{1}-2 \xi_{c s 1}^{1}\right) \xi_{c, \beta}^{1}+v_{s 1}\left(\xi_{s 1 c}^{1}-2 \xi_{s 1 s 1}^{1}\right) \xi_{s 1, \beta}^{1} \text { where } \beta=c, s 1 .
\end{aligned}
$$

The $\beta=c, s 1$ elementary currents $j_{\beta}^{\eta}$ and $j_{\beta}^{s}$, Eq. (181), contribute both to expectation values of the charge and spin current operators, respectively, of low-energy excited energy eigenstates and to off-diagonal matrix elements of such operators between the ground state and excited states of vanishing energy. The derivation in Ref. [93] of these elementary currents and charge and spin stiffnesses, Eq. (180), relies on the general procedures similar to those reported in Appendix E to obtain the corresponding expression of the zero-temperature spin stiffness of the spin-1/2 XXX chain.

The $\beta=c, s 1$ and $\alpha=\eta, s$ elementary currents $j_{\beta}^{\alpha}$ expressions, Eq. (181), can again be understood as being controlled by pseudoparticle parameters that play the same role as the Landau parameters in a Fermi liquid. On the one hand, the two-electron static quantities are expressed in terms of the $i=0$ symmetrical pseudoparticle Landau parameters in Eq. (176). On the other hand, the elementary charge and spin currents rather involve the $i=1$ anti-symmetrical pseudoparticle Landau parameters also given in that equation. The residual pseudoparticle interactions associated with the $f$ functions in that expression control the effects of the electronic interactions onto such two-electron quantities associated with charge and spin ballistic transport.

Specifically, the expression of the elementary currents, Eq. (181), in terms of the $\beta=c, s 1$ bands group velocities at a Fermi point and $i=1$ anti-symmetrical pseudoparticle Landau parameters read,

$$
\begin{aligned}
& j_{c}^{\eta}=v_{c} g_{c c}^{1} \text { and } j_{s 1}^{\eta}=v_{s 1} g_{s 1 c}^{1}, \\
& j_{c}^{s}=v_{c}\left(g_{c c}^{1}-2 g_{c s 1}^{1}\right) \text { and } j_{s 1}^{s}=-v_{s 1}\left(2 g_{s 1 s 1}^{1}-g_{s 1 c}^{1}\right) .
\end{aligned}
$$

In the $m \rightarrow 0$ and $m \rightarrow n_{e}$ spin-density limits and for electronic density in the range $n_{e} \in[0,1]$, these elementary currents have the following limiting behaviors,

$$
\begin{aligned}
& j_{c}^{\eta}=v_{c} \xi_{0}^{2}, \quad j_{s 1}^{\eta}=v_{c} \frac{\xi_{0}^{2}}{2}, j_{c}^{s}=0 \text { and } j_{s 1}^{s}=-v_{s 1} \text { for } m \rightarrow 0, \\
& j_{c}^{\eta}=v_{c}, \quad j_{s 1}^{\eta}=0, \quad j_{c}^{s}=v_{c} \text { and } j_{s 1}^{s}=0 \text { for } m \rightarrow n_{e} .
\end{aligned}
$$

By combining the stiffnesses expressions, Eq. (180), with the elementary-current limiting behaviors, Eq. (183), one finds that $D_{\eta}=v_{c} \xi_{0}^{2} /(2 \pi)$ at $m=0$. For $n_{e}<1$ and $m=0$, the charge stiffness $D_{\eta}$ changes from $D_{\eta}=(2 t / \pi) \sin (\pi n / 2)$ as $u \rightarrow 0$ to $D_{\eta}=(t / \pi) \sin \left(\pi n_{e}\right)$ for $u \gg 1$. For $m \rightarrow n_{e}$ the result is $D_{\eta}=(t / \pi) \sin \left(\pi n_{e}\right)$. At $n_{e}=1$ and $m=0$ one finds $D_{\eta}=2 t / \pi$ at $u=0$ and $D_{\eta}=0$ for $u>0$. This behavior stems from the $c$-band Fermi velocity $v_{c}$, Eq. (155) for $\beta=c$, being at $n_{e}=1$ finite at $u=0$ and vanishing for $u>0$. At $T=0$ the spin stiffness $D_{s}$ changes from $D_{s}=(2 t / \pi) \sin \left(\pi n_{e} / 2\right)$ for $u \rightarrow 0$ to $D_{s}=\left(t^{2} / U\right)\left(1-\sin \left(2 \pi n_{e}\right) /\left(2 \pi n_{e}\right)\right)$ for $u \gg 1$. For $m \rightarrow n_{e}$ one finds the expected result, $D_{s}=D_{\eta}=(t / \pi)$ $\sin \left(\pi n_{e}\right)$.

The charge stiffness $D_{\eta}$ and the spin stiffness $D_{s}$, Eq. (180), are plotted in Fig. 17 as a function of $U / t$ at electronic density $n_{e}=0.7$ and various values of the magnetic field $H$ in suitable units and as a function of $n_{e}$ for various values of the magnetic field $H$ and $U / t$. Such quantities are also plotted in Fig. 18 as a function of $U / t$ for magnetic field $H \rightarrow 0$ and electronic density $n_{e}=0.7$. In the right panel, the charge stiffness $D_{\eta}$ curves refer to several $n_{e}$ values.

Below in Section 7 it is shown that both the static properties and the 1D Hubbard model dynamical properties are controlled by the dressed phase shifts associated with the scattering events of the $\beta=c, s 1$ pseudofermions revisited in Section 7.1. 

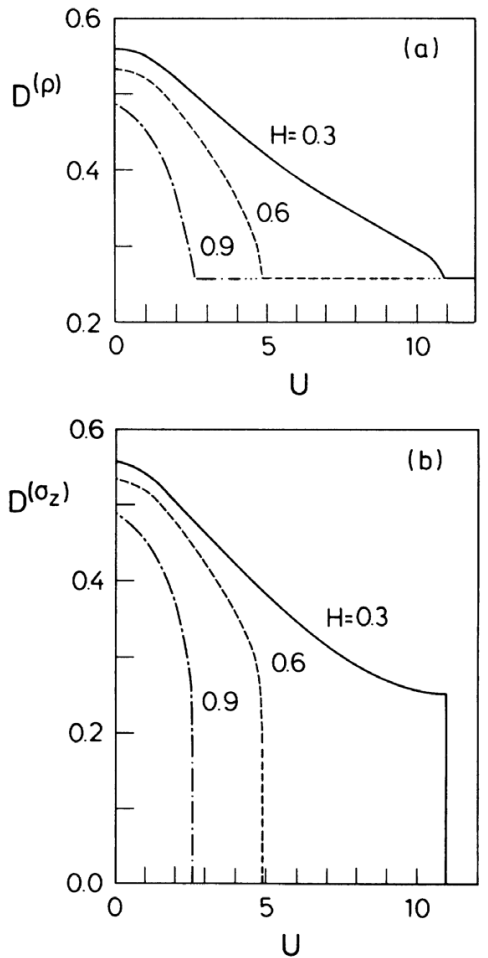
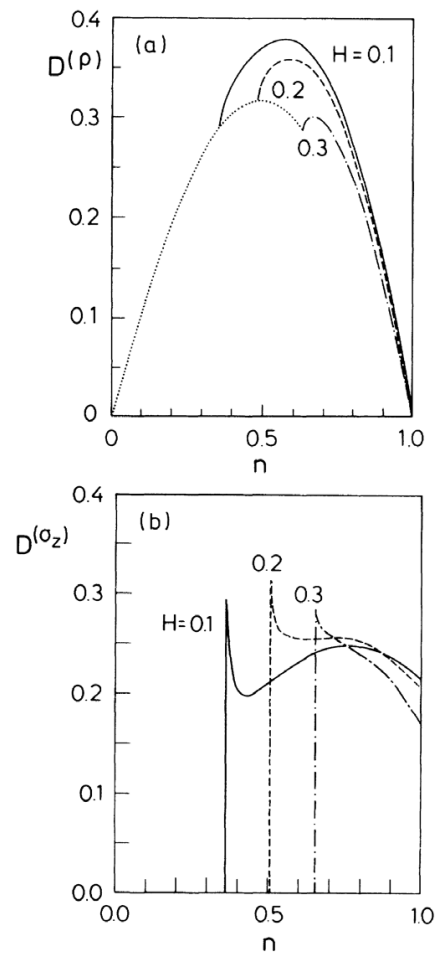
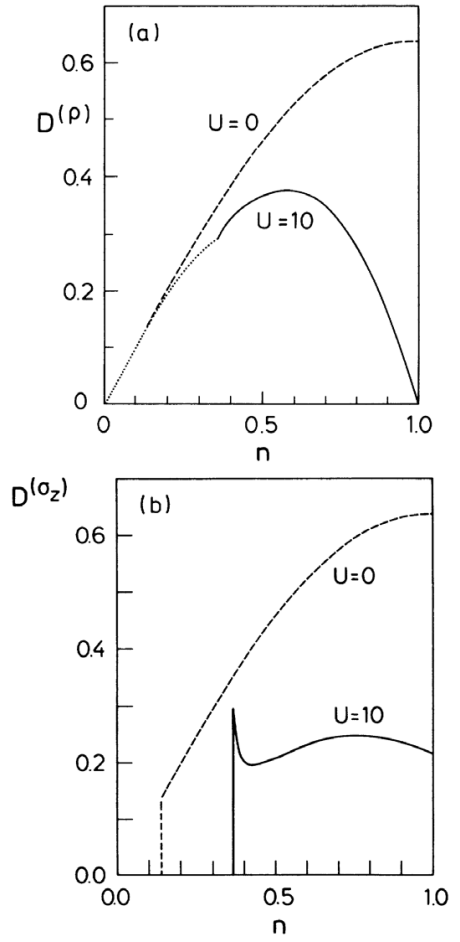

Fig. 17. The zero-temperature charge stiffness $D_{\eta}$ and the spin stiffness $D_{s}$, Eq. (180), versus $U / t$ at electronic density $n_{e}=0.7$ and values of the magnetic field $H=0.3$ (solid line), $H=0.6$ (dashed line), and $H=0.9$ (dashed-dotted line) in suitable units in the left panels, versus the electronic density $n_{e}$ for $U / t=10$ and values of the magnetic field $H=0.1$ (solid line), $H=0.2$ (dashed line), and $H=0.3$ (dashed-dotted line) in the middle panels, and versus the electronic density $n_{e}$ for $H=0.1$ and values of the on-site repulsion $U / t=0$ (dashed line) and $U / t=10$ (solid line) in the right panels. The discontinuities occur at $U / t$ or $n_{e}$ values at which according to Eq. (169) the magnetic field value $H$ under consideration becomes $H_{c}$ and thus the system becomes ferromagnetic. Due to the Mott-Hubbard insulator transition, $D_{\eta}$ is finite at $n_{e}=1$ when $U / t=0$ but vanishes when $U / t>0$. (As in Ref. [93], in the figures the charge stiffness $D_{\eta}$, spin stiffness $D_{s}$, and electronic density $n_{e}$ are denoted by $D^{(\rho)}, D^{\left(\sigma_{z}\right)}$, and $n$, respectively.)

Source: From Ref. [93].
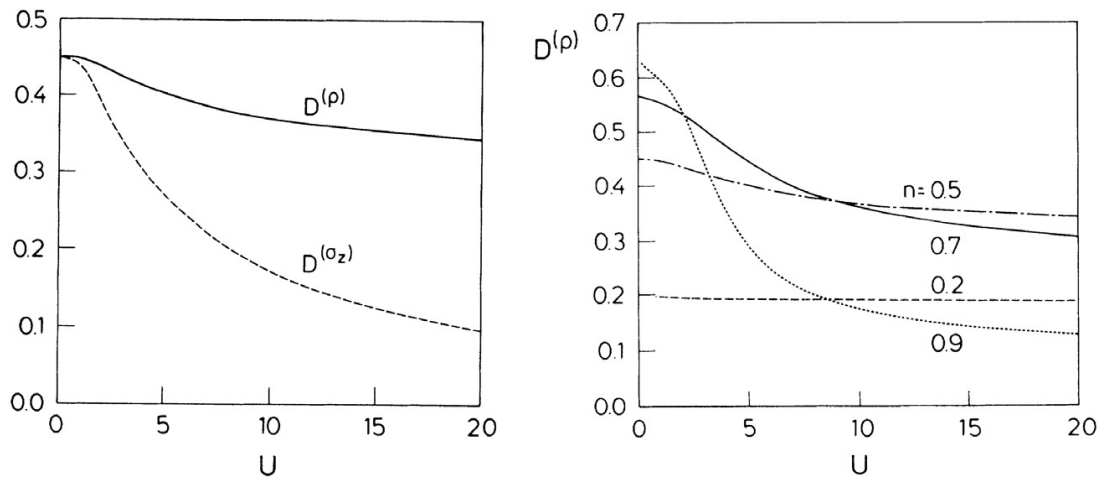

Fig. 18. The zero-temperature charge stiffness $D_{\eta}$ (left and right panels) and the spin stiffness $D_{s}$ (left panel), Eq. (180), versus $U / t$ for magnetic field $H \rightarrow 0$. The left and right panels figures are for electronic density $n_{e}=0.7$ and various $n_{e}$ values, respectively. Although $D_{\eta}$ has its maximum value for $n_{e} \rightarrow 1$, at $n_{e}=1$ it vanishes when $U / t>0$, yet is finite at $U / t=0$. This strong effect of the Mott-Hubbard insulator transition is also present for $0<H<H_{c}$. (As in Ref. [93], in the figures the charge stiffness $D_{\eta}$, spin stiffness $D_{s}$, and electronic density $n_{e}$ are denoted by $D^{(\rho)}$, $D^{\left(\sigma_{z}\right)}$, and $n$, respectively.) Source: From Ref. [93].

\subsection{Binding and anti-binding character of the spin-singlet pairs $(\alpha=s)$ and $\eta$-spin-singlet pairs $(\alpha=\eta)$}

From the use of the second expression in Eq. (158), the composite $\alpha n$ pseudoparticle energy dispersion, Eq. (152) for $\beta=\alpha n$, may be written as $\varepsilon_{\alpha n}\left(q_{j}\right)=n 2 \mu_{\alpha}+\varepsilon_{\alpha n}^{0}\left(q_{j}\right)$ where $\alpha=\eta, s$ and $n=1, \ldots, \infty$. The term $n 2 \mu_{\alpha}$ in this energy 
dispersion is merely additive in the intrinsic energy $2 \mu_{\alpha}=\varepsilon_{\alpha,-1 / 2}+\varepsilon_{\alpha,+1 / 2}$, Eq. (161), of two unpaired $\eta$-spins $1 / 2(\alpha=\eta)$ or two unpaired spins $1 / 2(\alpha=s)$ of opposite $\eta$-spin and spin projection, respectively.

Our aim is clarifying the anti-binding or binding character of the $\eta$-spin $(\alpha=\eta)$ and $\operatorname{spin}(\alpha=s) \alpha$-singlet configuration of two paired $\eta$-spins $1 / 2$ and two paired spins $1 / 2$, respectively. We first consider one single-pair $\alpha 1$ pseudoparticle for which $\varepsilon_{\alpha n}\left(q_{j}\right)=2 \mu_{\alpha}+\varepsilon_{\alpha 1}^{0}\left(q_{j}\right)$. The first energy term $2 \mu_{\alpha}$ gives the intrinsic energy of its two $\eta$-spins $1 / 2(\alpha=\eta)$ or spins $1 / 2(\alpha=s)$ of opposite projection if those were unpaired and in a $\alpha$-triplet $S_{\alpha}=1$ and $S_{\alpha}^{z}=0$ configuration. The second energy term $\varepsilon_{\alpha 1}^{0}\left(q_{j}\right)$ is thus a pairing energy. It refers to a binding or anti-binding character if $\varepsilon_{\alpha 1}^{0}\left(q_{j}\right)<0$ or $\varepsilon_{\alpha 1}^{0}\left(q_{j}\right)>0$, respectively. Analysis of the form of the $\alpha 1$ pseudoparticle energy dispersion, Eq. (152) for $\beta=\alpha 1$, then reveals that the spin-singlet $s 1$-pair configuration has a binding character. Indeed, it is such that $\varepsilon_{s 1}^{0}\left(q_{j}\right)<0$ for $\left|q_{j}\right|<q_{s 1}$, as confirmed by inspection of Fig. 11. As demonstrated by Fig. 13, the $\eta$-spin-singlet $\eta 1$-pair configuration is found in turn to have an anti-binding character. For it, $\varepsilon_{\eta 1}^{0}\left(q_{j}\right)>0$ for $\left|q_{j}\right|<q_{\eta 1}$. At the $\alpha=s, \eta$ limiting momenta $q_{j}= \pm q_{\alpha 1}$ one has though that $\varepsilon_{\alpha 1}^{0}\left( \pm q_{\alpha 1}\right)=0$. This means that for $q_{j} \rightarrow q_{\alpha 1}^{ \pm}= \pm q_{\alpha 1}$ the $\alpha$-singlet pair of a $\alpha 1$ pseudoparticle looses its binding or anti-binding character as its pairing energy vanishes.

Next we consider $\alpha n$-pairs configuration with $n>1$ pairs bound within it. One finds as well that $\varepsilon_{s n}^{0}\left(q_{j}\right)<0$ for $\left|q_{j}\right|<q_{s n}$ and $\varepsilon_{\eta n}^{0}\left(q_{j}\right)>0$ for $\left|q_{j}\right|<q_{\eta n}$ and $\varepsilon_{\alpha n}\left( \pm q_{\alpha n}\right)=0$ for $\alpha=\eta$, s. (See Fig. 12 for $\varepsilon_{s 2}^{0}\left(q_{j}\right)$ and Fig. 14 for $\varepsilon_{\eta 2}^{0}\left(q_{j}\right)$.) Irrespective of its binding or anti-binding character, the energy absolute value $\left|\varepsilon_{\alpha n}^{0}\left(q_{j}\right)\right|$ is here called $\alpha n$ pseudoparticle pairing energy. The strength of the $\alpha$-singlet pairs binding or anti-binding can be measured by the maximum reachable value of the $\alpha n$ pseudoparticle pairing energy upon creation of one $\alpha$ n pseudoparticle onto the ground state. Such a maximum value is reached at $q_{j}= \pm q_{F s 1}$ for the $s 1$ pseudoparticles and at $q_{j}=0$ for all other $\alpha n \neq s 1$ pseudoparticles,

$$
W_{s 1}^{\text {pair }}=\left|\varepsilon_{s 1}^{0}\left(q_{F s 1}\right)\right|=W_{s 1}^{h}=2 \mu_{B} H \text { and } W_{\alpha n}^{\text {pair }}=\left|\varepsilon_{\alpha n}^{0}(0)\right|=W_{\alpha n} \text { for } \alpha n \neq s 1 .
$$

Here $W_{s 1}^{h}=2 \mu_{B} H$ is the ground-state energy bandwidth of the $s 1$ band hole unoccupied part and $W_{\alpha n}$ is the energy bandwidth of $\alpha n \neq s 1$ momentum bands.

The maximum pairing energy $W_{s n}^{\text {pair }}$ vanishes at $H=0$. This is because then $W_{s 1}^{h}=2 \mu_{B} H=0$ and the energy dispersion $\varepsilon_{s n}^{0}\left(q_{j}\right)$ and its momentum bandwidth vanish at $H=0$ for $n>1$. Similarly, the maximum pairing energy $W_{\eta n}^{\text {pair }}$ vanishes at electronic density $n_{e}=1$. Indeed, the energy dispersion $\varepsilon_{\eta n}^{0}\left(q_{j}\right)$ and its momentum bandwidth vanish at $n_{e}=1$. The latter maximum $\eta n$ pseudoparticle pairing energy is given in Eq. (B.9) of Appendix B in the $u \rightarrow 0$ and $u \gg 1$ limits for electronic densities $\left.n_{e} \in\right] 0,1$ [ and spin density $m=0$. For the electronic density interval $\left.n_{e} \in\right] 0,1\left[\right.$ and spin density $m \rightarrow n_{e}$, one has that $q_{F s 1} \rightarrow 0$. The maximum pairing energies $W_{s n}^{\text {pair }}=W_{s n}=\left|\varepsilon_{s n}^{0}(0)\right|$ and $W_{\eta n}^{\text {pair }}=W_{\eta n}=\left|\varepsilon_{\eta n}^{0}(0)\right|$ have for these densities analytical expressions that are functions of $n_{e}$ and $U / t$. They are given in Eqs. (B.10) and (B.11) of Appendix B. In the $u \rightarrow 0$ and $u \gg 1$ limits these expressions simplify, as given in Eqs. (B.12) and (B.13) of that Appendix.

Actually, the suitable energy scale to measure the strength of the binding or anti-binding pairing is the maximum value of the $\alpha n$ pseudoparticle pairing energy per pair, $\pi_{\alpha n}^{\text {pair }} \equiv W_{\alpha n}^{\text {pair }} / n$. Consistently with the values and expressions given in Eqs. (B.9)-(B.13) of Appendix B, one finds that the energy per pair $\pi_{\alpha n}^{\text {pair }}$ is for $n>1$ always smaller than $\pi_{\alpha 1}^{\text {pair }}$. For densities $\left.n_{e} \in\right] 0,1\left[\right.$ and $m \in\left[0, n_{e}\right]$ it has the limiting behaviors,

$$
\pi_{\alpha n}^{\text {pair }}=\pi_{\alpha 1}^{\text {pair }} / n \text { for } u \rightarrow 0 \text { and } \pi_{\alpha n}^{\text {pair }}=\pi_{\alpha 1}^{\text {pair }} / n^{2} \text { for } u \gg 1 .
$$

It obeys the inequality $\pi_{\alpha 1}^{\text {pair }} / n^{2} \leq \pi_{\alpha n}^{\text {pair }} \leq \pi_{\alpha 1}^{\text {pair }} / n$ for the whole $u>0$ range. Hence the energy per pair $\pi_{\alpha n}^{\text {pair }}$ decreases upon increasing $n$. This effect is stronger upon increasing $u$. This reveals that the overall binding of the $n>1$ pairs within an $\alpha n$-pairs configuration tends to suppress the binding $(\alpha=s)$ and anti-binding $(\alpha=\eta)$ energy within each such pairs. This suppression is an increasing function of both the number of pairs $n$ and of $u$.

In Appendix I the relation of the maximum pairing energy $W_{\alpha n}^{\text {pair }}$ and the $\alpha n$ pseudoparticle effective mass $m_{\alpha n}^{*}$ in Eq. (I.1) of that Appendix to $\eta$-spin $(\alpha=\eta)$ and spin $(\alpha=s) \delta S_{\alpha}= \pm n$ multiplet excitations is discussed. This refers to electronic densities in the interval $\left.n_{e} \in\right] 0,1\left[\right.$, spin density $m \rightarrow n_{e}$, and the whole $u>0$ range. The quantities $W_{\alpha n}^{\text {pair }}$ and $m_{\alpha n}^{*}$ are found to be related yet different quantities.

\section{Dynamical correlation functions in the pseudofermion representation}

Here we revisit the $\beta$ pseudofermion representation and shortly consider the corresponding PDT. This includes its applications to the 1D Hubbard model in a magnetic field. In addition, the relation between the PDT and the MQIM $[52,53]$ is clarified.

\subsection{The pseudofermion representation}

One finds from the use of the TBA equations, Eqs. (128) and (129), that for PS excited states the $\beta=c, \alpha n$ rapidity functionals $\Lambda^{\beta}\left(q_{j}\right)$ can be written in terms of the corresponding ground-state rapidity functions $\Lambda_{0}^{\beta}\left(q_{j}\right)$ as follows,

$$
\Lambda^{c}\left(q_{j}\right)=\Lambda_{0}^{c}\left(\bar{q}\left(q_{j}\right)\right)=\sin k_{0}^{c}\left(\bar{q}\left(q_{j}\right)\right) \text { for } j=1, \ldots, L \text { and } \Lambda^{\alpha n}\left(q_{j}\right)=\Lambda_{0}^{\alpha n}\left(\bar{q}\left(q_{j}\right)\right) \text { for } j=1, \ldots, L_{\alpha n} .
$$



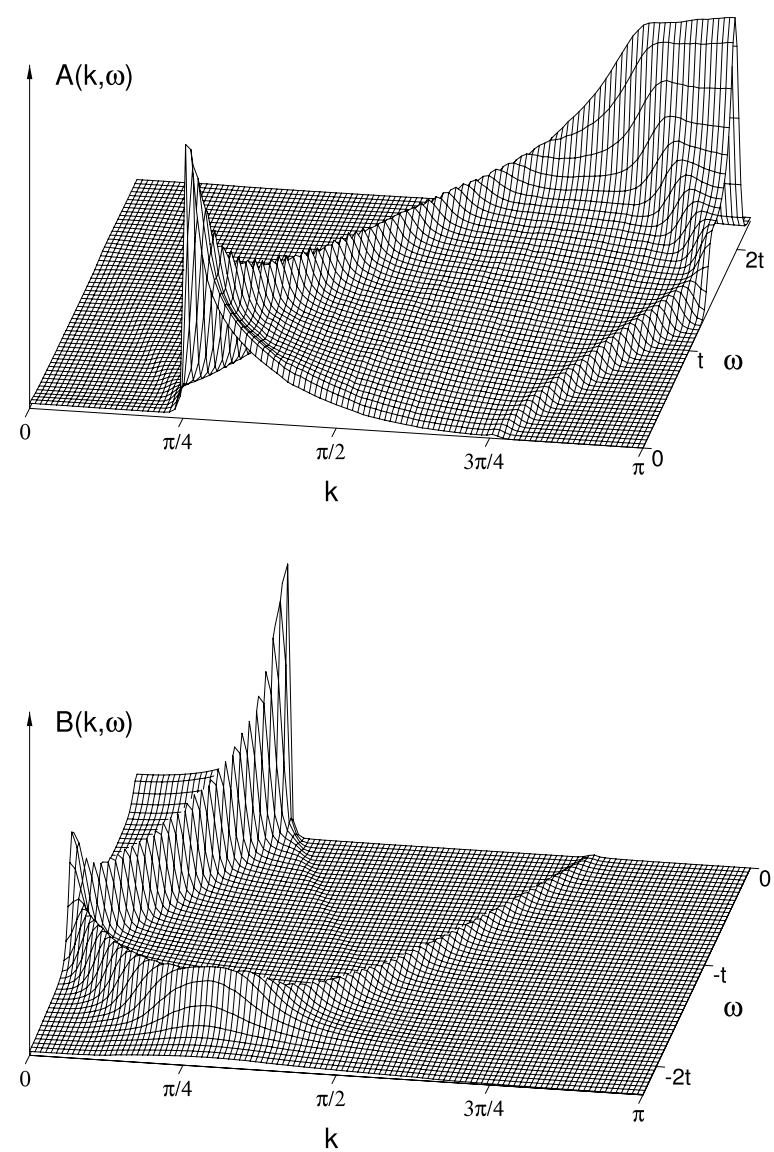

Fig. 19. One-electron removal $(\omega<0)$ and addition $(\omega>0)$ spectral functions over the whole $(k, \omega)$ plane for $u \gg 1, n_{e}=0.5$, and $m=0$. Source: From Ref. [157].

Here $\bar{q}_{j}=\bar{q}\left(q_{j}\right)$ where $j=1, \ldots, L_{\beta}$ are the following discrete canonical momentum values,

$$
\bar{q}_{j}=\bar{q}\left(q_{j}\right)=q_{j}+\frac{2 \pi \Phi_{\beta}\left(q_{j}\right)}{L}=\frac{2 \pi}{L} I_{j}^{\beta}+\frac{2 \pi \Phi_{\beta}\left(q_{j}\right)}{L} \text { for } j=1, \ldots, L_{\beta} \text { and } \beta=c, \alpha n .
$$

(The excited-states rapidity expression, Eq. (186), is similar to that of the simpler models considered in Sections 2 and 3.) The functional $2 \pi \Phi_{\beta}\left(q_{j}\right)$ in Eq. (187) reads [51,268],

$$
2 \pi \Phi_{\beta}\left(q_{j}\right)=\sum_{\beta^{\prime}} \sum_{j^{\prime}=1}^{L_{\beta^{\prime}}} 2 \pi \Phi_{\beta \beta^{\prime}}\left(q_{j}, q_{j^{\prime}}\right) \delta N_{\beta^{\prime}}\left(q_{j^{\prime}}\right) .
$$

Here the deviation $\delta N_{\beta^{\prime}}\left(q_{j^{\prime}}\right)$ and the dressed phase shift $2 \pi \Phi_{\beta \beta^{\prime}}\left(q_{j}, q_{j^{\prime}}\right)$ are those in Eq. (148) and Eq. (154), respectively. The discrete canonical momentum values $\bar{q}_{j}=\bar{q}\left(q_{j}\right)$ have spacing $\bar{q}_{j+1}-\bar{q}_{j}=2 \pi / L+$ h.o.. (h.o. stands here for terms of second order in $1 / L$.)

We associate one $\beta$ pseudofermion with each of the $N_{\beta}$ occupied $\beta$-band discrete canonical momentum values $\bar{q}_{j}$ $[13,51,58,59,63,64]$. We associate one $\beta$ pseudofermion hole with each of the remaining $N_{\beta}^{h}$ unoccupied $\beta$-band discrete canonical momentum values $\bar{q}_{j}$ of a PS excited state. There is a pseudofermion representation for each ground state and its PS. This holds for $u>0$ and all electronic and spin densities. The chosen initial ground state plays the role of vacuum of the pseudofermion representation. For it one has that $\bar{q}_{j}=q_{j}$ in Eq. (187). This also occurs for the $\alpha n \neq s 1$ bands of the PS excited states of a $S_{\alpha}=0$ ground state. For the latter state the number of $\alpha n \neq s 1$ band discrete momentum values $L_{\alpha n}$, Eq. (130), vanishes, $L_{\alpha n}=0$. For it the functional, Eq. (188), also vanishes, $2 \pi \Phi_{\alpha n}\left(q_{j}\right)=0$. It follows that $\bar{q}_{j}=q_{j}$ for the $\alpha n \neq s 1$ bands of its PS excited states.

In Sections 4.3 and 5.2, $\beta=c, s 1$ pseudoparticle creation and annihilation operators $f_{q_{i}, \beta}^{\dagger}$ and $f_{q_{j}, \beta}$, respectively, have been introduced. The corresponding $\beta=c, s 1$ pseudofermions play a major role in the PDT. Their operators read,

$$
f_{\bar{q}_{j}, \beta}^{\dagger}=f_{q_{j}+2 \pi \Phi_{\beta}\left(q_{j}\right) / L, \beta}^{\dagger}=\left(\hat{S}_{\beta}^{\Phi}\right)^{\dagger} f_{q_{j}, \beta}^{\dagger} \hat{S}_{\beta}^{\Phi} \text { for } f_{\bar{q}_{j}, \beta}=\left(f_{\bar{q}_{j}, \beta}^{\dagger}\right)^{\dagger} \text { for } \beta=c, s 1 .
$$


Here $\hat{S}_{\beta}^{\Phi}$ denotes the $\beta$ pseudoparticle- $\beta$ pseudofermion unitary operator, $\hat{S}_{\beta}^{\Phi}=e^{\sum_{j=1}^{L_{\beta}} f_{q_{j}+(2 \pi / L) \Phi_{\beta}\left(q_{j}\right), \beta}^{\dagger} f_{q_{j}, \beta}}$. It is such that $\left(\hat{S}_{\beta}^{\Phi}\right)^{\dagger}=e^{\sum_{j=1}^{L_{\beta}} f_{q_{j}-(2 \pi / L) \Phi_{\beta}\left(q_{j}\right), \beta}^{\dagger} f_{q_{j}, \beta}}$.

The functional $2 \pi \Phi_{\beta}\left(q_{j}\right)$, Eq. (188), has an important physical meaning: It is the overall scattering phase shift acquired by a $\beta$ pseudofermion or $\beta$ pseudofermion hole of initial-state canonical momentum $q_{j}$ upon scattering off all $\beta^{\prime}$ pseudofermions and/or $\beta^{\prime}$ pseudofermion holes created under a transition from the ground state to one of its PS excited states. As confirmed below in Section 7.2, that scattering phase shift controls the spectral weights of dynamical correlation functions.

It then follows from the form of the functional expression in Eq. (188) that within the pseudofermion scattering theory [268], the function $2 \pi \Phi_{\beta \beta^{\prime}}\left(q_{j}, q_{j^{\prime}}\right)$, Eq. (154), (and $\left.-2 \pi \Phi_{\beta \beta^{\prime}}\left(q_{j}, q_{j^{\prime}}\right)\right)$ has a well-defined physical meaning. It is the phase shift acquired by a $\beta$ pseudofermion or $\beta$ pseudofermion hole of initial-state canonical momentum $\bar{q}_{j}=q_{j}$ upon scattering off a $\beta^{\prime}$ pseudofermion (and a $\beta^{\prime}$ pseudofermion hole) created at the canonical momentum $\bar{q}_{j^{\prime}}=\bar{q}\left(q_{j^{\prime}}\right)$ corresponding to the initial ground-state momentum $q_{j^{\prime}}$ under a transition from that state to one of its PS excited states. This reveals that all physical quantities whose expression was shown in Section 6 to depend on the phase-shift parameters, Eq. (162), are controlled by pseudofermion scattering events.

Upon expressing the PS energy functional, Eq. (151), in terms of the discrete canonical momentum values $\bar{q}_{j}=\bar{q}\left(q_{j}\right)$, Eq. (187), it reads $\delta E=\sum_{\beta} \sum_{j=1}^{L_{\beta}} \varepsilon_{\beta}\left(\bar{q}_{j}\right) \delta \mathcal{N}_{\beta}\left(\bar{q}_{j}\right)+\sum_{\alpha=\eta, s} \varepsilon_{\alpha,-1 / 2} M_{\alpha,-1 / 2}$ up to $\mathcal{O}(1 / L)$ order. (This is as in the case of the 1D Lieb-Liniger Bose gas, Eq. (35).) The $\beta$ pseudofermion energy dispersions $\varepsilon_{\beta}\left(\bar{q}_{j}\right)$ in that functional expression have exactly the same form as those given in Eq. (152) with the momentum $q_{j}$ replaced by the corresponding canonical momentum, $\bar{q}_{j}=\bar{q}\left(q_{j}\right)$. The energy functional applying to the pseudofermions thus has no energy interaction terms of second-order in the deviations $\delta \mathcal{N}_{\beta}\left(\bar{q}_{j}\right)$. This is in contrast to the equivalent energy functional, Eq. (151). Indeed, up to $\mathcal{O}(1 / L)$ order the $\beta$ pseudofermions have no such interactions. Such a property allows the dynamical correlation functions to be expressed as a convolution of $c$ and $s 1$ pseudofermion spectral functions. Such functions spectral weights can be expressed as Slater determinants written in terms of anticommutators of pseudofermion operators.

That within the present representation the $\beta$ pseudofermion scattering phase shifts $2 \pi \Phi_{\beta}\left(q_{j}\right)$ are incorporated in the canonical momentum, Eq. (187), has though consequences on the form of such Slater determinants. Those involve the type of $\beta$ pseudofermion operators anticommutators given in Eq. (J.1) of Appendix J. The unitarity of the $\beta$ pseudoparticle $-\beta$ pseudofermion transformation preserves the pseudoparticle operator algebra provided that the canonical momentum values $\bar{q}_{j}$ and $\bar{q}_{j^{\prime}}$ belong to the $\beta$ band of the same energy and momentum eigenstate. The exotic form of the anticommutator, Eq. (J.1) of Appendix J, follows from shake-up effects stemming from $\bar{q}_{j}$ and $\bar{q}_{j^{\prime}}$ corresponding in it to the excited-state and ground-state $\beta$ band, respectively. Such an exotic $\beta$ pseudofermion operator algebra plays an important role in the one- and two-electron high-energy spectral weight distributions $[59,181]$ of the PDT reviewed in the ensuing section.

\subsection{The pseudofermion dynamical theory}

The goal of this section is revisiting the PDT and thus to illustrate how the microscopic mechanisms that control the dynamical and spectral properties are much simpler to describe in terms of pseudofermion processes than of the underlying many-particle interactions.

It has been difficult to apply the BA to the derivation of high-energy dynamical correlation functions. For the 1D Hubbard model, the method employed in Refs. [156-158] has been the first breakthrough to address that problem in the $u \rightarrow \infty$ limit. In such references the one-electron spectral functions have been derived for the whole $(k, \omega)$ plane by accounting for the phase shifts imposed on the spinless-fermions by the XXX chain spins. (See Fig. 19). Such fractionalized particles naturally arise from the $u \rightarrow \infty N_{e}$-electron wave-function factorization [119,150]. The related PDT relies on the corresponding factorization of the finite- $u N_{e}$-rotated-electron wave function. This issue is addressed in Appendix F. The PDT involves an extension of the $u \rightarrow \infty$ method of Refs. [156-158] to the whole finite- $u$ range. This theory has been the first breakthrough for the derivation of analytical expressions of the 1D Hubbard model high-energy spectral functions for that extended onsite repulsion range $[13,16,51,58,59,63,64,181]$.

In the following we consider the $\beta=c, \alpha$ n pseudofermions of the 1D Hubbard model. We indicate as well the small differences relative to the simplified PDT suitable to the other models under review. The aim of the theory is the evaluation of finite- $\omega$ one- and two-particle dynamical correlation functions of general form,

$$
B(k, \omega)=\sum_{f}|\langle f|\hat{O}(k)| G S\rangle|^{2} \delta\left(\omega-\left(E_{f}-E_{G S}\right)\right) \text { for } \omega>0 .
$$

Here $\hat{O}(k)$ is a one- or two-particle operator, $|G S\rangle$ a ground state, and $|f\rangle$ its excited states contained in $\hat{O}(k)|G S\rangle$.

The elementary processes that generate PS excited states contained in $\hat{O}(k)|G S\rangle$ from the ground state can be classified into the following three (A)-(C) classes [13,16,51,58,59,63,181]: (A) High-energy and finite-momentum processes that besides creation or annihilation of $c$ and $s 1$ pseudofermions may involve the creation of $\alpha n \neq s 1$ pseudofermions and/or unpaired $\eta$-spins of projection -1/2,(B) zero-energy and finite-momentum processes that conserve the number of $\beta=c, s 1$ pseudofermions yet change their number at the $\iota=+1$ right and $\iota=-1$ left $\beta=c, s 1$ Fermi points, and (C) low-energy and small-momentum elementary $\beta=c, s 1$ pseudofermion particle-hole processes in the vicinity of the right $(\iota=+1)$ and 
left $(\iota=+1) \beta=c, s 1$ Fermi points onto the momentum occupancy configurations generated by the elementary processes (A) and (B).

For a momentum $k$ and a given small energy range around $\omega$, the excitation $\hat{O}(k)|G S\rangle$ can be written as a sum of terms, $\sum_{i} \hat{O}_{i}^{\odot}(k)|G S\rangle$. Here $i=0,1,2, \ldots$ refers to a suitable index. Its value for each specific operator $\hat{O}(k)$ can be uniquely defined in terms of the increasing number of pseudofermions created and annihilated by processes (A) and (B) under the transitions to the excited states. $\hat{O}_{i}^{\circ}(k)$ is the corresponding generator onto the ground state of such processes of momentum $k$ and energy $\omega$. (Further information about the expansions $\sum_{i} \hat{O}_{i}^{\odot}(k)|G S\rangle$ of specific physical operators $\hat{O}(k)$ and the choice of the corresponding leading-order operators $\hat{O}^{\odot}(k)$ can be found in Section 3.1 of Ref. [63] for two-particle spin operators and in Section 3.2 of Ref. [64] for one-electron operators.)

For a well-defined small energy range around each low- or high-energy $\omega$, one approximates the dynamical correlation function, Eq. (190), by a corresponding leading-order term [13,16,51,58,59,63,64,181],

$$
\begin{aligned}
B(k, \omega) & \approx B^{\odot}(k, \omega)=\sum_{f}\left|\left\langle f\left|\hat{O}^{\odot}(k)\right| G S\right\rangle\right|^{2} \delta\left(\omega-\left(E_{f}-E_{G S}\right)\right) \\
& =\sum_{f} \Theta\left(\Omega-\delta \omega_{f}\right) \Theta\left(\delta \omega_{f}\right) \Theta\left(\left|v_{f}\right|-v_{\bar{\beta}}\right) \breve{B}_{f}^{\odot}\left(\delta \omega_{f}, v_{f}\right) \text { for } \omega>0 .
\end{aligned}
$$

Here

$$
\breve{B}_{f}^{\odot}\left(\delta \omega_{f}, v_{f}\right)=\frac{\operatorname{sgn}\left(v_{f}\right)}{2 \pi} \int_{0}^{\delta \omega_{f}} d \omega^{\prime} \int_{-\operatorname{sgn}\left(v_{f}\right) \delta \omega_{f} / v_{\beta}}^{+\operatorname{sgn}\left(v_{f}\right) \delta \omega_{f} / v_{\beta}} d k^{\prime} B_{Q_{\bar{\beta}}}\left(\delta \omega_{f} / v_{f}-k^{\prime}, \delta \omega_{f}-\omega^{\prime}\right) B_{Q_{\beta}}\left(k^{\prime}, \omega^{\prime}\right),
$$

and

$$
\begin{aligned}
B_{Q_{\beta}}\left(k^{\prime}, \omega^{\prime}\right) & =\frac{L}{2 \pi} \sum_{m_{\beta,+1} ; m_{\beta,-1}} A_{\beta}^{(0,0)} a_{\beta}\left(m_{\beta,+1}, m_{\beta,-1}\right) \\
& \times \delta\left(\omega^{\prime}-\frac{2 \pi}{L} v_{\beta} \sum_{\iota= \pm 1}\left(m_{\beta, l}+\Delta_{\beta}^{\iota}\right)\right) \delta\left(k^{\prime}-\frac{2 \pi}{L} \sum_{\iota= \pm 1} \iota\left(m_{\beta, \iota}+\Delta_{\beta}^{\iota}\right)\right) \text { where } \beta=c, s 1 .
\end{aligned}
$$

The quantities in these equations are defined below.

In the function $B^{\odot}(k, \omega)$ initial general expression, the generator onto the particle vacuum of the ground state $|G S\rangle$ is written in terms of $\beta$ pseudofermion creation operators. Their $\beta$ band discrete canonical momentum values, which equal the corresponding momentum values $q_{j}$, Eqs. (131) and (132), are those of that ground state. Both the generator onto the electron vacuum of the PS excited states $|f\rangle$ and the operator $\hat{O}^{\odot}(k)$ are written in terms of $\beta$ pseudofermion operators. Their $\beta$ band discrete canonical momentum values $\bar{q}_{j}$, Eq. (187), are those of the excited states.

The summation $\sum_{f}$ in Eq. (191) runs over PS excited states generated by processes (A), (B), and (C) at fixed values of $k$ and $\omega$. The capital- $\Theta$ distribution $\Theta(x)$ in that equation is given here and in the following by $\Theta(x)=1$ for $x \geq 0$ and $\Theta(x)=0$ for $x<0$. Such states have excitation energy and momentum in the ranges $\delta E_{f}^{\odot} \in[\omega-\Omega, \omega]$ and $\delta P_{f}^{\odot} \in\left[k-\Omega / v_{f}, k\right]$, respectively, where $\Omega$ stands for the processes (C) energy range. Moreover, in Eqs. (191) and (192) the fixed excitation energy $\omega$ and momentum $k$ read $\omega=\delta E_{f}^{\odot}+\delta \omega_{f}$ and $k=\delta P_{f}^{\odot}+\delta k_{f}$, respectively. Here $\delta \omega_{f}=\left(\omega-\delta E_{f}^{\odot}\right)=\left(\omega-E_{f}^{\odot}+E_{G S}\right)$, $\delta k_{f}=k-\delta P_{f}^{\odot}, v_{f}=\delta \omega_{f} / \delta k_{f}, v_{\bar{\beta}}=\min \left\{v_{c}, v_{s 1}\right\}, v_{\beta}=\max \left\{v_{c}, v_{s 1}\right\}$, and $v_{c}$ and $v_{s 1}$ are the $\beta=c, s 1$ band Fermi velocities, Eq. (155). The above processes (C) energy range $\Omega$ is self-consistently determined as that for which the velocity $v_{f}$ remains nearly unchanged.

The lack of $c$ and $s 1$ pseudofermion energy interactions in their PS $u>0$ spectrum is behind the function $\breve{B}_{f}^{\odot}\left(\delta \omega_{f}, v_{f}\right)$ in Eq. (191) being expressed in Eq. (192) as a convolution of $c$ and $s 1$ pseudofermion spectral functions [51,181]. Such functions expression, Eq. (193), involves sums that run over the processes (C) numbers $m_{\beta, \iota}=1,2,3, \ldots[13,59,181]$. In it $\Delta_{\beta}^{\iota}$ refers to the four dimensions functionals $2 \Delta_{\beta}^{\iota}=\left(\delta \bar{q}_{F \beta}^{\iota} /[2 \pi / L]\right)^{2}$. Those are the four $\beta=c, s 1$ and $\iota= \pm$ relative weights, Eq. (J.5) of Appendix J. They correspond to the smallest finite processes (C) numbers, $m_{\beta, l}=1$, and can be written as,

$$
2 \Delta_{\beta}^{\iota}=\left(\sum_{\beta^{\prime}=c, s 1}\left(\iota \xi_{\beta \beta^{\prime}}^{0} \frac{\delta N_{\beta^{\prime}}^{F}}{2}+\xi_{\beta \beta^{\prime}}^{1} \delta J_{\beta^{\prime}}^{F}+\sum_{j=1}^{L_{\beta^{\prime}}} \Phi_{\beta \beta^{\prime}}\left(\iota q_{F \beta}, q_{j}\right) \delta N_{\beta^{\prime}}^{N F}\left(q_{j}\right)\right)+\sum_{\alpha n \neq s 1} \sum_{j=1}^{L_{\alpha n}} \Phi_{\beta \alpha n}\left(\iota q_{F \beta}, q_{j}\right) \delta N_{\alpha n}\left(q_{j}\right)\right)^{2} .
$$

Here $\beta=c, s 1, \delta N_{\beta^{\prime}}^{F}=\delta N_{\beta^{\prime},+}^{F}+\delta N_{\beta^{\prime},-}^{F}$, and $2 J_{\beta^{\prime}}^{F}=\delta N_{\beta^{\prime},+}^{F}-\delta N_{\beta^{\prime},-}^{F}$ for $\beta^{\prime}=c, s 1$. Moreover, the $\beta=c, s 1$ lowest peak weight $A_{\beta}^{(0,0)}$ in Eq. (193) is associated with transitions from the ground state to PS excited states generated by processes (A) and (B). The $\beta=c, s 1$ relative weight $a_{\beta}=a_{\beta}\left(m_{\beta,+1}, m_{\beta,-1}\right)$ is generated by additional processes (C). The former weight refers to a Slater determinant that involves the $\beta=c, s 1$ pseudofermion anticommutators, Eq. (J.1) of Appendix J. The lowest peak weight $A_{\beta}^{(0,0)}$ and the relative weight $a_{\beta}$ are given in Eq. (J.2) and Eqs. (J.3)-(J.5) of that Appendix, respectively.

The PDT has a simplified form suitable to the 1D Lieb-Liniger Bose gas [61], spin-1/2 XXX chain [62], and spin-spin dynamical correlation functions of the 1D half-filled Hubbard model [63]. Such functions involve spin excitations for which $N_{c}^{h}=0$. For that simplified dynamical theory there is no convolution, as given in Eq. (192). For it the function, Eq. (191), 
rather reads [61-63] $B^{\odot}(k, \omega)=\sum_{f} \Theta\left(\left|v_{f}\right|-v\right) B_{Q}\left(\delta \omega_{f}, v_{f}\right)$ for $\delta \omega_{f} \in[0, \Omega]$. Here $v=v\left(q_{F}\right)$ is the model-dependent ground-state single branch Fermi velocity and $B_{Q}\left(k^{\prime}, \omega^{\prime}\right)$ is a pseudofermion spectral function. It has exactly the expression, Eq. (193), if one omits the index $\beta$. Such an omission procedure also applies to all other PDT quantities in the equations given in the following and in Appendix J [61-63].

The expression, Eq. (J.10) of Appendix J, of the $\beta=c, s 1$ pseudofermion spectral function, Eq. (193), is valid in the TL. Its use in the general convolution expression, Eq. (192), of the function $\breve{B}_{f}^{\odot}\left(\delta \omega_{f}, v_{f}\right)$ followed by the use of the obtained expression for such a function in the second expression of the function $B^{\odot}(k, \omega)$ in Eq. (191), enables performing the summations in the latter equation for the $(k, \omega)$-plane vicinity of some singular spectral features. For the one-electron spectral function, these turn out to be the most important spectral features.

The summation $\sum_{f}$ in Eq. (191) runs over PS excited states with the specific $k$ and $\omega$ values that appear in the argument of the corresponding function $B^{\odot}(k, \omega)$. At such fixed values, the two corresponding $\beta=c, s 1$ lowest peak weights $A_{\beta}^{(0,0)}$, Eq. (J.2) of Appendix J, have nearly the same magnitude for all such states. In the vicinity of the $\beta=c, s 1$ branch lines whose spectrum is defined in the following, the state summations can then be partially performed. One then finds that near them the spectral function behaves as $[13,16,51,58,59,63,64,181]$,

$$
\begin{aligned}
B(k, \omega) & \propto\left(c_{B} \omega-\omega_{\beta}(k)\right)^{\zeta_{\beta}(k)} \text { for }\left(c_{B} \omega-\omega_{\beta}(k)\right) \geq 0, \\
\zeta_{\beta}(k) & =-1+\left.\sum_{\beta^{\prime}=c, s 1} \sum_{l= \pm} 2 \Delta_{\beta^{\prime}}^{\iota}\left(q_{j}\right)\right|_{q_{j}=c_{0}\left(k-k_{0}\right)} \text { where } \beta=c, s 1 .
\end{aligned}
$$

Here $c_{B}=1$ and $c_{B}=-1$ for $\omega \geq 0$ and $\omega \leq 0$ excitation energy, respectively. Except for the one-electron removal spectral function, for which $c_{B}=-1$, the general convention is that $c_{B}=1$ and thus $\omega \geq 0$. (That in Eq. (195) the $\beta=c, s 1$ branch line spectrum $\omega_{\beta}(k)$ is not multiplied by $c_{B}$ is justified by it being according to Eq. (196) always such that $\omega_{\beta}(k) \geq 0$.)

A $\beta=c, s 1$ branch line in the vicinity of which the expression, Eq. (195), applies results from transitions to excited states generated by creation $\left(c_{0}=+1\right)$ or annihilation $\left(c_{0}=-1\right)$ of one $\beta=c, s 1$ pseudofermion. Its canonical momentum $\bar{q}_{j}=\bar{q}\left(q_{j}\right)$ is associated with a uniquely defined $\beta=c, s 1$ band momentum value $q_{j}$. A $c_{0}=+1$ and $c_{0}=-1$ branch line corresponds to the range $\left|q_{j}\right| \in\left[q_{F \beta}, q_{\beta}\right]$ and $q_{j} \in\left[-q_{F \beta}, q_{F \beta}\right]$, respectively. All remaining $\beta=c, s 1$ pseudofermions are created or annihilated at the $\beta=c, s 1$ Fermi points $\pm q_{F \beta}$, Eq. (147). The $\beta=\alpha n \neq s 1$ pseudofermions (if any) are created at the $\beta=\alpha n \neq s 1$ band limiting values, $q_{\alpha n}^{ \pm}= \pm q_{\alpha n}$, Eq. (149). This gives a $(k, \omega)$-plane $\beta=c, s 1$ branch line shape whose energy spectrum $\omega_{\beta}(k)$ appearing in expression, Eq. (195), reads [51],

$$
\omega_{\beta}(k)=\omega_{0}+c_{0} \varepsilon_{\beta}\left(q_{j}\right) \text { and } k=k_{0}+c_{0} q_{j} \text { where } \beta=c, s 1 \text { and } c_{0}= \pm 1 .
$$

$\varepsilon_{\beta}\left(q_{j}\right)$ is here the $\beta=c, s 1$ band energy dispersion, Eq. (152), and,

$$
\omega_{0}=\sum_{\alpha=\eta, s} 2 \mu_{\alpha}\left(L_{\alpha,-1 / 2}-\delta_{\alpha, s} N_{s 1}\right) \text { and } k_{0}=\pi L_{\eta,-1 / 2}+\left(\pi-2 k_{F}\right) 2 J_{\eta n}+\sum_{\beta=c, s 1} 2 q_{F \beta} 2 J_{\beta}^{F} .
$$

$2 J_{\eta n}=N_{\eta n,+}-N_{\eta n,-}$ where $N_{\eta n, \iota}$ is in this equation the number of $\eta n$ pseudofermions created at the $\eta n$ band limiting momentum values $\iota q_{\eta n}=\iota\left(\pi-2 k_{F}\right)$, Eq. (149). For instance, $\left(N_{\eta 1, \iota}+M_{\eta,-1 / 2}\right)=0$ and $\left(N_{\eta n, 1, \iota}+M_{\eta,-1 / 2}\right)=1$ for a oneelectron addition $\beta=c, s 1$ branch line in the lower and upper Hubbard bands, respectively, defined below in Section 7.3.

The spectral function expression, Eq. (195), is exact for $\beta=c, s 1$ branch lines that coincide with the lower thresholds $\left(c_{B}=1\right)$ or upper thresholds $\left(c_{B}=-1\right)$ of $(k, \omega)$-plane finite spectral-weight regions. For the particular case of the oneelectron spectral function, Eq. (195) is a good approximation for the $\beta=c, s 1$ branch lines that have a small amount of spectral weight above $\left(c_{B}=1\right)$ or below $\left(c_{B}=-1\right)$ them. For integrable correlated problems with a single pseudofermion branch, the exponent in Eq. (195) is rather given by $-1+\sum_{l= \pm} 2 \Delta^{\iota}$. This is as in Eqs. (46) and (98) for the 1D Lieb-Liniger Bose gas and spin-1/2 XXX chain, respectively [61-63].

The above dynamical correlation functions line shapes are beyond the reach of the techniques used within the usual lowenergy TLL studies. In the limit of low-energy the PDT describes the well-known behaviors obtained by such techniques. This refers specifically to the vicinity of $(k, \omega)$-plane points $\left(k_{0}, 0\right)$ of which $\left(k_{0}, \omega_{0}\right)$, Eq. (197), is a generalization for $\omega_{0}>0$. Near them the spectral-function behavior is $[51,63,64,181]$,

$$
\begin{aligned}
B(k, \omega) & \propto\left(c_{B} \omega-\omega_{0}\right)^{\zeta} \text { for }\left(c_{B} \omega-\omega_{0}\right) \geq 0, \\
\zeta & =-2+\sum_{\beta^{\prime}=c, s 1} \sum_{l= \pm} 2 \Delta_{\beta^{\prime}}^{l} \text { for }\left(c_{B} \omega-\omega_{0}\right) \neq \pm v_{\beta}\left(k-k_{0}\right) \text { where } \beta=c, s 1, \\
B(k, \omega) & \propto\left(c_{B} \omega-\omega_{0} \mp v_{\beta}\left(k-k_{0}\right)\right)^{\zeta^{ \pm}} \text {for }\left(c_{B} \omega-\omega_{0} \mp v_{\beta}\left(k-k_{0}\right)\right) \geq 0, \\
\zeta^{ \pm} & =-1-2 \Delta_{\beta}^{\mp}+\sum_{\beta^{\prime}=c, s 1} \sum_{l= \pm} 2 \Delta_{\beta^{\prime}}^{l} \text { for }\left(c_{B} \omega-\omega_{0}\right) \approx \pm v_{\beta}\left(k-k_{0}\right) \text { where } \beta=c, s 1 .
\end{aligned}
$$

The expressions given here apply to the finite-weight region above $\left(c_{B}=1\right)$ or below $\left(c_{B}=-1\right)$ the $(k, \omega)$-plane point. Examples of exponents $\zeta$ controlling the line shape near $(k, \omega)$-plane points $\left(k_{0}, 0\right)$ are given in Ref. [100]. In Ref. [181] it is 


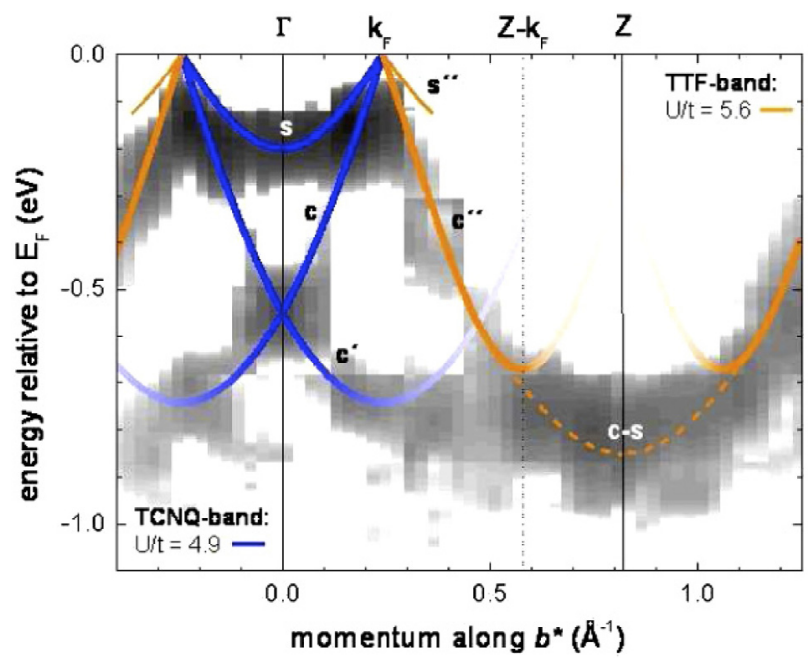

Fig. 20. Experimental peak dispersions obtained by ARPES on TTF-TCNQ along the easy-transport axis as given in Fig. 7 of Ref. [15] and matching theoretical branch and border lines, within the 1D Hubbard model PDT. The line shape in the vicinity of the branch lines is for that model of power-law type, with exponents that depend on the momentum, interaction strength, and densities. (The Z-point corresponds in the figure to the momentum $k=\pi$.) Source: From Ref. [13].

confirmed that in the limit of low excitation energy the expressions, Eq. (198), recover those provided within the TLL limit by conformal-field theory [176,224]. For low-energy excited eigenstates of $S_{\eta}>0$ and $S_{s} \geq 0$ ground states the four functionals $2 \Delta_{c}^{ \pm 1}$ and $2 \Delta_{s 1}^{ \pm 1}$, Eq. (194) with $\delta N_{\beta^{\prime}}^{N F}\left(q_{j}\right)=0$ and $\delta N_{\alpha n}\left(q_{j}\right)=0$, are in that reference found to be the conformal dimensions of the $c, \pm$ and $s, \pm$ primary fields, respectively. The corresponding dressed charge matrix is in Eqs. (162) and (163) expressed in terms of pseudofermion phase-shift parameters.

In the particular case of the one-electron spectral function, there is a third type of high-energy spectral feature in the vicinity of which the PDT provides an analytical expression. It is generated by processes where a $c$ pseudofermion hole is annihilated (electron addition) or created (electron removal) and a $s 1$ pseudofermion hole is created at related momentum values $q_{j}$ and $q_{j^{\prime}}$, respectively. Their relation follows from the group velocities, Eq. (155), obeying the equality $v_{c}\left(q_{j}\right)=v_{s 1}\left(q_{j^{\prime}}\right)$. The one-electron spectral feature under consideration is called a $c-s 1$ border line. Its $(k, \omega)$-plane shape is of the general form $[13,51,63,64]$,

$$
\omega_{c-s 1}(k)=\left(\omega_{0}+\left|\epsilon_{c}\left(q_{j}\right)\right|+\left|\epsilon_{s 1}\left(q_{j^{\prime}}\right)\right|\right) \delta_{v_{c}\left(q_{j}\right), v_{s 1}\left(q_{j^{\prime}}\right)} \text { and } k=k_{0}+c_{0} q_{j}-q_{j^{\prime}} \text { where } c_{0}= \pm 1 .
$$

Near a $c-s 1$ border line the spectral function has the following behavior,

$$
B(k, \omega) \propto\left(c_{B} \omega-\omega_{c-s 1}(k)\right)^{-1 / 2} \text { for }\left(c_{B} \omega-\omega_{c-s 1}(k)\right) \geq 0 .
$$

Applications of the 1D Hubbard model PDT to the study of the ARPES spectral features of actual quasi-1D materials are reported in Refs. $[13,15,16]$. The experimental peak dispersions obtained by ARPES on the quasi-1D organic conductor TTFTCNQ along the easy-transport axis [15] together with the prediction of the PDT for the 1D Hubbard model are shown in Fig. 20. The shape of the $c, c^{\prime}$, and $c^{\prime \prime}$ spectral lines and $s$ and $s^{\prime \prime}$ spectral lines in the ARPES spectrum plotted in such a figure is that of the $\beta=c$ and $\beta=s 1$ bands energy dispersions $\varepsilon_{\beta}\left(q_{j}\right)$, Eq. (195), respectively, in the branch-line spectrum, Eq. (196). The indices $s$ and $s^{\prime \prime}$ read within our notation $s 1$ and $s 1^{\prime}$, respectively. The $c, c^{\prime}$, and $s 1$ branch lines refer to electronic densities in the range $n_{e} \in[0,1]$ suitable to the stacks of TCNQ molecules related spectral features. Their spectra expressions in terms of the energy dispersions $\varepsilon_{c}\left(q_{j}\right)$ and $\varepsilon_{s 1}\left(q_{j}\right)$ and PDT momentum dependent exponents are given in Eqs. (J.11) and (J.12) of Appendix J, respectively. Such dispersions are seen in actual experiments on quasi-1D conductors.

The figure theoretical $c^{\prime \prime}$ and $s^{\prime \prime}$ branch lines and $c-s$ border line, Eq. (199), refer to the TTF stack of molecules spectral features derived within the 1D Hubbard model PDT for electronic density $n_{e}=1.41$ and $U / t=5.61$. The $c, s$, and $c^{\prime}$ branch lines correspond to the TCNQ stack of molecules dispersions evaluated for electronic density $n_{e}=0.59$ and $U / t=4.90$. A corresponding approximate spectral-weight distribution in the vicinity of such branch lines obtained by combining the theory analytical expressions with numerical approximations is shown in Fig. 21.

The one-electron spectral function exponent plotted in Fig. 22 refers for $k \in\left[0, k_{F}\right]$ to a line shape of form, Eq. (195). Above it there is no one-electron removal spectral weight $\left(c_{B}=-1\right)$. Hence it is exact for the present model. It corresponds to the theoretical TCNQ line called $s$ in Fig. 20. The one-electron addition exponent plotted in Fig. 22 for $k \in\left[k_{F}, 3 k_{F}\right]$ and electronic density $n_{e}=0.59$ equals the corresponding one-electron removal exponent for density $n_{e}^{\prime}=2-n_{e}=1.41$. The 


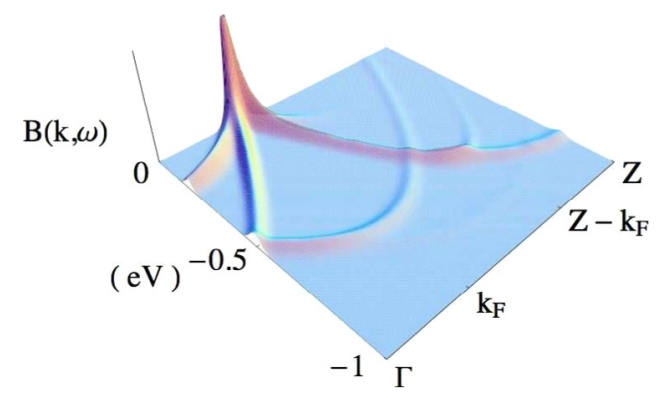

Fig. 21. PDT one-electron removal spectral-weight distribution in the vicinity of the $\operatorname{TTF}\left(n_{e}=1.41\right.$ and $\left.U / t=5.61\right)$ and TCNQ $\left(n_{e}=0.59\right.$ and $\left.U / t=4.90\right)$ stack of molecules spectral features in Fig. 20. Source: From Ref. [13].

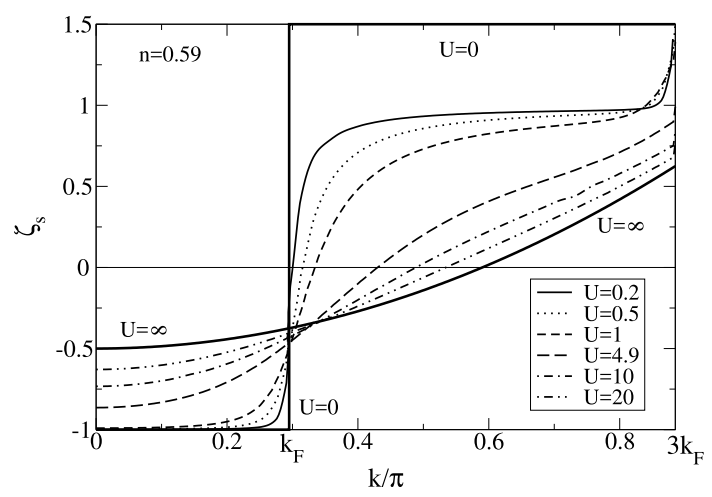

Fig. 22. Momentum dependence of a one-electron spectral function $s 1$ branch-line exponent, Eq. (195), called $\zeta_{s}$ in the figure, for densities $n_{e}=0.59$ and $m=0$ and several $U / t$ values. (As in Ref. [16], $n_{e}$ is here denoted by $n$.)

Source: From Ref. [16].

latter is that appropriate for the theoretical TTF $s^{\prime \prime}$ branch line plotted in Fig. 20 for the small range of momentum values $k>k_{F}$ for which that exponent is negative.

The experimental peak dispersions shown in Fig. 20 show significant discrepancies from the conventional band-structure predictions. Fig. 7 of Ref. [15] represents the experimental spectral features in that figure in comparison with the conduction band dispersions obtained by density functional theory. In contrast to the line shapes obtained within the 1D Hubbard model by the PDT, those predicted by density functional theory do not agree with the experimental ARPES features. The corresponding non-perturbative many-electron physics justifies why standard density functional theory fails to describe such unusual ARPES spectral-line shapes. The theoretical description of the microscopic mechanisms behind the spectral properties of 1D systems and quasi-1D metals can be further improved by the use of a renormalized PDT [26]. It accounts for the effects of electron finite-range interactions beyond the conventional 1D Hubbard model.

The results discussed in this section and in Section 6 illustrate how the 1D Hubbard model physics is fully controlled by the scattering events of the pseudofermions. The model one-particle spectral functions has also been studied by numerical methods. The authors of Ref. [269] found that the 1D Hubbard model one-electron removal spectral function $s 1$ branch line exact exponent plotted in Fig. 22 for the momentum range $k \in\left[0, k_{F}\right]$ fully agrees with that exponent values obtained by the density matrix renormalization group (DMRG). The PDT exponent for the line shape near the $c$ branch line in Fig. 20 is not exact. Indeed, there is some small amount of spectral weight above that line. It is though a very good approximation. Consistently, the authors of Ref. [269] have found small minor quantitative deviations from the DMRG values of that exponent.

The numerical results derived by the MQIM in Section VIII of Ref. [54] for the momentum dependence of the one-electron removal spectral-function $s 1$ branch line exponent of Fig. 22 are in full quantitative agreement with those obtained by use of the PDT $[13,16,58]$ for electronic density $n_{e}=0.59$, interaction values $U / t=1.00,4.90,10.00$. This applies to the whole range of that figure momentum values $k \in\left[0, k_{F}\right]$ associated with electron removal. Moreover, the same exponent was also calculated in the framework of the MQIM in Ref. [55], using input from the BA solution. It has been plotted in that reference as a function of the momentum for densities $n_{e}=0.17,0.25$, and $U / t=2.00,5.00,10.00,20.00$. Again, such results are in accord with those of the PDT. 

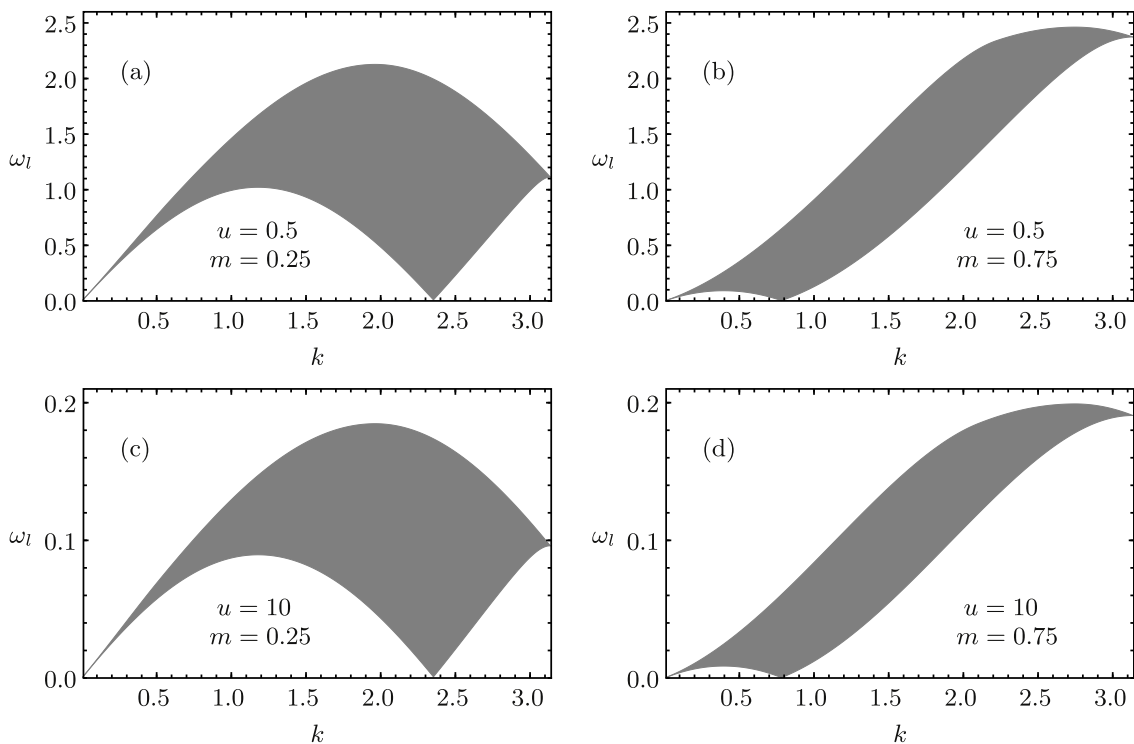

Fig. 23. The half-filled 1D Hubbard model longitudinal spin spectrum $\omega^{l}(k)$ corresponding to that plotted in Figs. 7-9 for the spin-1/2 XXX chain for (a) $m=0.25$ and $u=0.5$, (b) $m=0.75$ and $u=0.5$, (c) $m=0.25$ and $u=10.0$, and (d) $m=0.75$ and $u=10.0$. The main effect of the onsite repulsion is on the spectrum energy bandwidth. At fixed spin density $m$ its form remains nearly the same for the whole $u>0$ range.

Source: From Ref. [63].

\subsection{PDT applications to the high-energy spectral properties of the 1D Hubbard model in a magnetic field}

The longitudinal and transverse dynamical structure factors studied in Section 3.7 for the spin- $1 / 2 X X X$ chain and the oneelectron spectral functions have been investigated within the framework of the 1D Hubbard model in a magnetic field PDT in Refs. [63] and [64], respectively. The results for the XXX chain correspond to those obtained for the large- $u$ half-filled 1D Hubbard model up to $t^{2} / U$ order. Previous studies of the factors $S^{z z}(k, \omega)$ and $S^{x x}(k, \omega)=S^{y y}(k, \omega)$ within the half-filled 1D Hubbard focused mainly onto magnetic fields $H=0$ for which $S^{z z}(k, \omega)=S^{x x}(k, \omega)=S^{y y}(k, \omega)[270,271]$. That model lower thresholds spectra $\omega^{l}(k)$ and $\omega^{t}(k)$ of the longitudinal and transverse, respectively, dynamical structure factors are plotted in Figs. 23 and 24, respectively. Interestingly, analysis of these figures reveals that the main effect of $u$ on these spectra is merely on their energy bandwidth. It increases upon decreasing $u$. Otherwise, their shape remains nearly unchanged.

The exact behavior near the longitudinal and transverse dynamical structure factors lower thresholds refers to the PDT general expression, Eq. (195). The corresponding singularities in the vicinity of the thresholds of the longitudinal spin spectrum $\omega^{l}(k)$ in Fig. 23 and transverse spin spectrum $\omega^{t}(k)$ in Fig. 24 are controlled by exponents $\xi^{l}(k)$ and $\xi^{t}(k)$, respectively. Such exponents are plotted for the half-filled 1D Hubbard model as a function of the momentum $k \in] 0, \pi$ [ for several values of $u$ and spin density $m$ in Figs. 25 and 26, respectively.

On the one hand, the longitudinal dynamical structure factor exponent $\xi^{l}(k)$ is negative for $k>0$ at any $u$ and $m$ values. On the other hand, the transverse dynamical structure factor exponent $\xi^{t}(k)$ is negative for a $u$ and $m$-dependent range $k \in\left[k_{t}, \pi\right]$ where the momentum $k_{t}$ is for $u>0$ an increasing function of $m$. Furthermore, analysis of Fig. 25 reveals that the negative exponent $\xi^{l}(k)$ is an increasing and decreasing function of $u$ for the momentum ranges $k \in\left[0, k_{l}\right]$ and $k \in\left[k_{l}, \pi\right]$, respectively. Here $k_{l}$ is a spin density dependent momentum at which the exponent $\xi^{l}(k)$ has similar value for the whole $u>0$ range.

For the intervals of the momentum $k$ for which the exponent $\xi_{\tau}(k)$ is negative, there are lower threshold singularity cusps in the dynamical structure factors. Hence analysis of Figs. 25 and 26 provides valuable information on the $k$ ranges for which there are singularities in the lower thresholds of the dynamical structure factors $S^{z z}(k, \omega)$ and $S^{x x}(k, \omega)=S^{y y}(k, \omega)$. An interesting issue discussed in Section 3.7 for the spin-1/2 XXX chain is the potential observation of the theoretically predicted dynamical structure factors peaks in inelastic neutron scattering experiments. Analysis of Figs. 24-26 reveals that the effect of lessening the $u$ value is enhancing the $(k, \omega)$-plane energy bandwidths of the spectra edges where such peaks are located.

An interesting issue refers to the effects of varying the electronic density $n_{e}$, spin density $m$, and interaction $u$ on the momentum dependence of the exponents that control the $(k, \omega)$-plane singular features of the $\sigma=\uparrow, \downarrow$ one-electron spectral functions $B_{\sigma, \gamma}(k, \omega)$ of the 1D Hubbard model [64]. Here $\gamma=-1$ (and $\left.\gamma=+1\right)$ for one-electron removal (and addition). Such functions read,

$$
B_{\sigma,-1}(k, \omega)=\sum_{v^{-}}\left|\left\langle v^{-}\left|c_{k, \sigma}\right| G S\right\rangle\right|^{2} \delta\left(\omega+\left(E_{v^{-}}^{N_{\sigma}-1}-E_{G S}^{N_{\sigma}}\right)\right) \text { for } \omega \leq 0,
$$



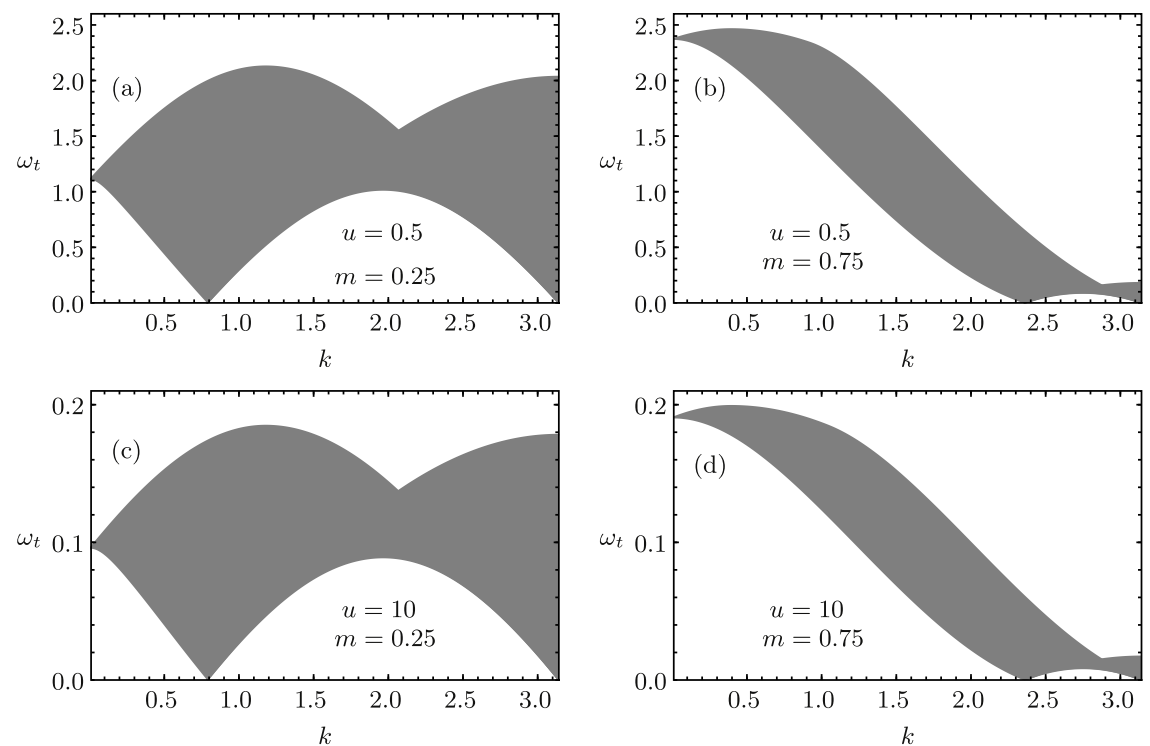

Fig. 24. The half-filled 1D Hubbard model transverse spin spectrum $\omega^{t}(k)$ corresponding to that plotted in Figs. 7-9 for the spin-1/2 $X X X$ chain for (a) $m=0.25$ and $u=0.5$, (b) $m=0.75$ and $u=0.5$, (c) $m=0.25$ and $u=10.0$, and (d) $m=0.75$ and $u=10.0$. As in the case of the longitudinal spin spectrum plotted in Fig. 23, the main effect of the onsite repulsion is on the spectrum energy bandwidth.

Source: From Ref. [63].
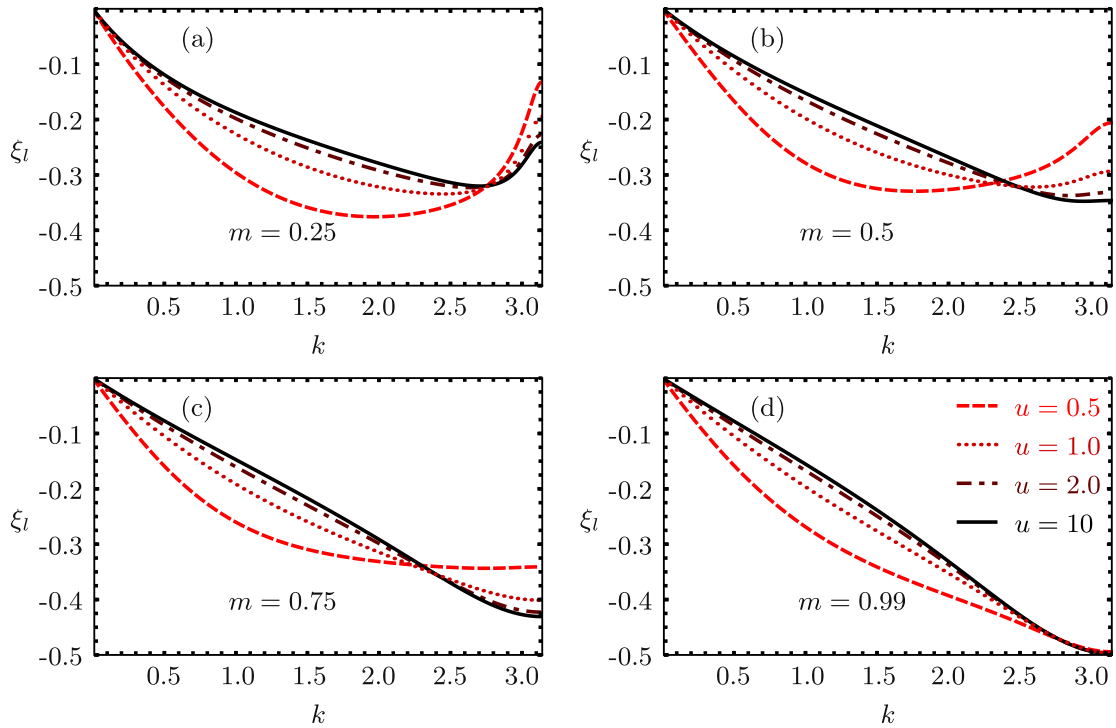

Fig. 25. The half-filled 1D Hubbard model exponent $\xi^{l}(k)$ corresponding to that plotted in Figs. 7-9 for the spin-1/2 XXX chain that controls the singularities in the vicinity of the lower thresholds of the longitudinal spin spectrum $\omega^{l}(k)$ plotted in Fig. 23 as a function of $\left.k \in\right] 0$, $\pi$ [ for several values of $u$ and spin densities (a) $m=0.25$, (b) $m=0.50$, (c) $m=0.75$, and (d) $m=0.99$.

Source: From Ref. [63].

$$
B_{\sigma,+1}(k, \omega)=\sum_{v^{+}}\left|\left\langle v^{+}\left|c_{k, \sigma}^{\dagger}\right| G S\right\rangle\right|^{2} \delta\left(\omega-\left(E_{v^{+}}^{N_{\sigma}+1}-E_{G S}^{N_{\sigma}}\right)\right) \text { for } \omega \geq 0 .
$$

The operators $c_{k, \sigma}$ and $c_{k, \sigma}^{\dagger}$ in this equation annihilate and create electrons, respectively, of momentum $k$ and $|G S\rangle$ denotes the initial $N_{\sigma}$-electron ground state of energy $E_{G S}^{N_{\sigma}}$. The $v^{-}$and $v^{+}$summations run over the $N_{\sigma}-1$ and $N_{\sigma}+1$-electron excited energy eigenstates, respectively, and $E_{v^{-}}^{N_{\sigma}-1}$ and $E_{v^{+}}^{N_{\sigma}+1}$ are the corresponding energies.

The one-electron lower Hubbard band and upper Hubbard band can be defined for all densities and finite repulsive onsite interaction values in terms of the rotated electrons associated with the model BA solution [64]. As discussed in Section 4.3, 

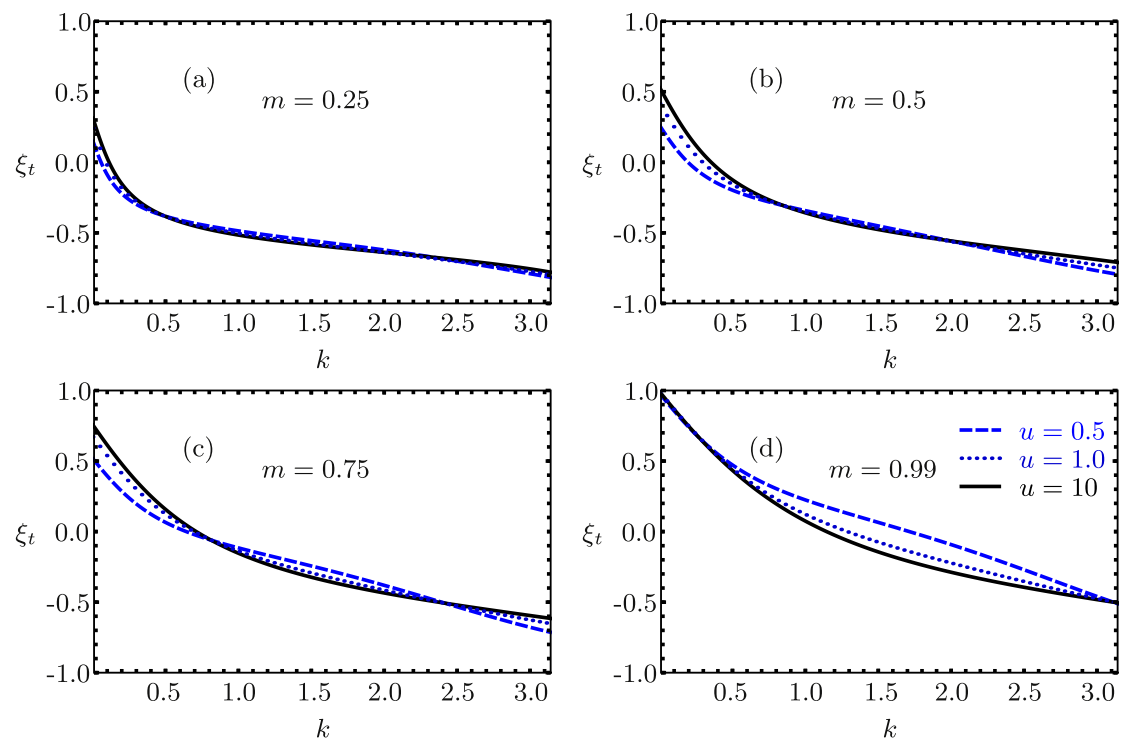

Fig. 26. The half-filled 1D Hubbard model exponent $\xi^{t}(k)$ corresponding to that plotted in Figs. 7-9 for the spin-1/2 XXX chain that controls the singularities in the vicinity of the lower thresholds of the transverse spin spectrum $\omega^{t}(k)$ plotted in Fig. 24 as a function of $k \in 10, \pi[$ for several values of $u$ and spin densities (a) $m=0.25$, (b) $m=0.50$, (c) $m=0.75$, and (d) $m=0.99$.

Source: From Ref. [63].

the 1D Hubbard model BA quantum numbers are directly related to the numbers of sites singly occupied, doubly occupied, and unoccupied by $\sigma$ rotated electrons. From the use of that relation it is found that for instance for electronic densities $n_{e} \in\left[0,1\left[\right.\right.$ and spin densities $m \in\left[0, n_{e}\right]$ the model ground states have zero rotated-electron double occupancy. The $\sigma$ one-electron LHB addition spectral function $B_{\sigma,+1}^{\mathrm{LHB}}(k, \omega)$ and $\mathrm{UHB}$ addition spectral function $B_{\sigma,+1}^{\mathrm{UHB}}(k, \omega)$ are then uniquely defined for $u>0$ as $B_{\sigma,+1}(k, \omega)=B_{\sigma,+1}^{\mathrm{LHB}}(k, \omega)+B_{\sigma,+1}^{\mathrm{UHB}}(k, \omega)$ where $B_{\sigma,+1}^{\mathrm{LHB}}(k, \omega)=\sum_{v_{0}^{+}}\left|\left\langle v_{0}^{+}\left|c_{k, \sigma}^{\dagger}\right| G S\right\rangle\right|^{2} \delta\left(\omega-\left(E_{v_{0}^{+}}^{N_{\sigma}+1}-E_{G S}^{N_{\sigma}}\right)\right)$ for $\omega \geq 0$ and $B_{\sigma,+1}^{\mathrm{UHB}}(k, \omega)=\sum_{v_{D}^{+}}\left|\left\langle v_{D}^{+}\left|c_{k, \sigma}^{\dagger}\right| G S\right\rangle\right|^{2} \delta\left(\omega-\left(E_{v_{D}^{+}}^{N_{\sigma}+1}-E_{G S}^{N_{\sigma}}\right)\right)$ for $\omega \geq 0$. Here the $v_{0}^{+}$and $v_{D}^{+}$summations run over the $N_{\sigma}+1$-electron excited energy eigenstates with zero and $D>0$, respectively, rotated-electron double occupancy and $E_{v_{0}^{+}}^{N_{\sigma}-1}$ and $E_{v_{D}^{+}}^{N_{\sigma}+1}$ are the corresponding energies.

The momentum dependent exponents that control the line shape of the $\sigma=\uparrow, \downarrow$ one-electron spectral functions $B_{\sigma, \gamma}(k, \omega)$, Eq. (201), near the main $c$ and $s 1$ branch lines are in Ref. [64] plotted as a function of $k$ for a large range of different $u, n_{e}$, and $m$ values. For simplicity, here we plot some of the momentum dependent exponents of the down-spinone-electron removal and LHB addition spectral function, Eq. (201) for $\sigma=\downarrow$ and $\gamma=-1$. Such a spectral function $c^{ \pm}$branch lines and $s 1$ branch line are generated by processes that correspond to particular cases of those generated by the leadingorder operators considered in Ref. [64]. These branch lines energy spectra are defined in that reference. The corresponding momentum dependent exponents $\xi_{c^{+}}^{\downarrow}(k)=\xi_{c^{-}}^{\downarrow}(-k)$ and $\xi_{s 1}^{\downarrow}(k)$ that control the spectral-function singularities in the vicinity of the $c^{+}$and $s 1$ branch lines are plotted in Figs. 27 and 28, respectively. The effects of varying $u$ on these one-electron exponents are stronger than in the case of the exponents of the spin dynamical structure factors plotted in Figs. 25 and 26.

\subsection{Relation between the pseudofermion dynamical theory and the mobile quantum impurity model}

For simplicity, here we use the PDT expressions for the dynamical correlation functions of the 1D Lieb-Liniger Bose gas discussed in Section 2.2 to clarify the relation between the PDT and the MQIM [52,53]. The basic relation found in the following is qualitatively similar to that of the more complex models also reviewed in this paper.

Within the MQIM, the pseudoparticles in the vicinity of the $\iota= \pm$ Fermi points are called particles. The MQIM relies on an effective Hamiltonian of general form [52,53],

$$
\begin{aligned}
\hat{H} & =\hat{H}_{0}+\hat{H}_{d}+\hat{H}_{\text {int }} \text { where } \hat{H}_{0}=\frac{v}{2 \pi} \int d x\left(K_{0}(\nabla \theta(x))^{2}+\frac{1}{K_{0}}(\nabla \phi(x))^{2}\right), \\
\hat{H}_{d} & =\int d x d^{\dagger}(x)\left(\varepsilon_{1}(k)-i \frac{\partial \varepsilon_{1}(k)}{\partial k} \frac{\partial}{\partial x}\right) d(x), \\
\hat{H}_{\text {int }} & =\int d x\left(V_{R} \nabla \frac{\theta(x)-\phi(x)}{2 \pi}-V_{L} \nabla \frac{\theta(x)+\phi(x)}{2 \pi}\right) d^{\dagger}(x) d(x) .
\end{aligned}
$$



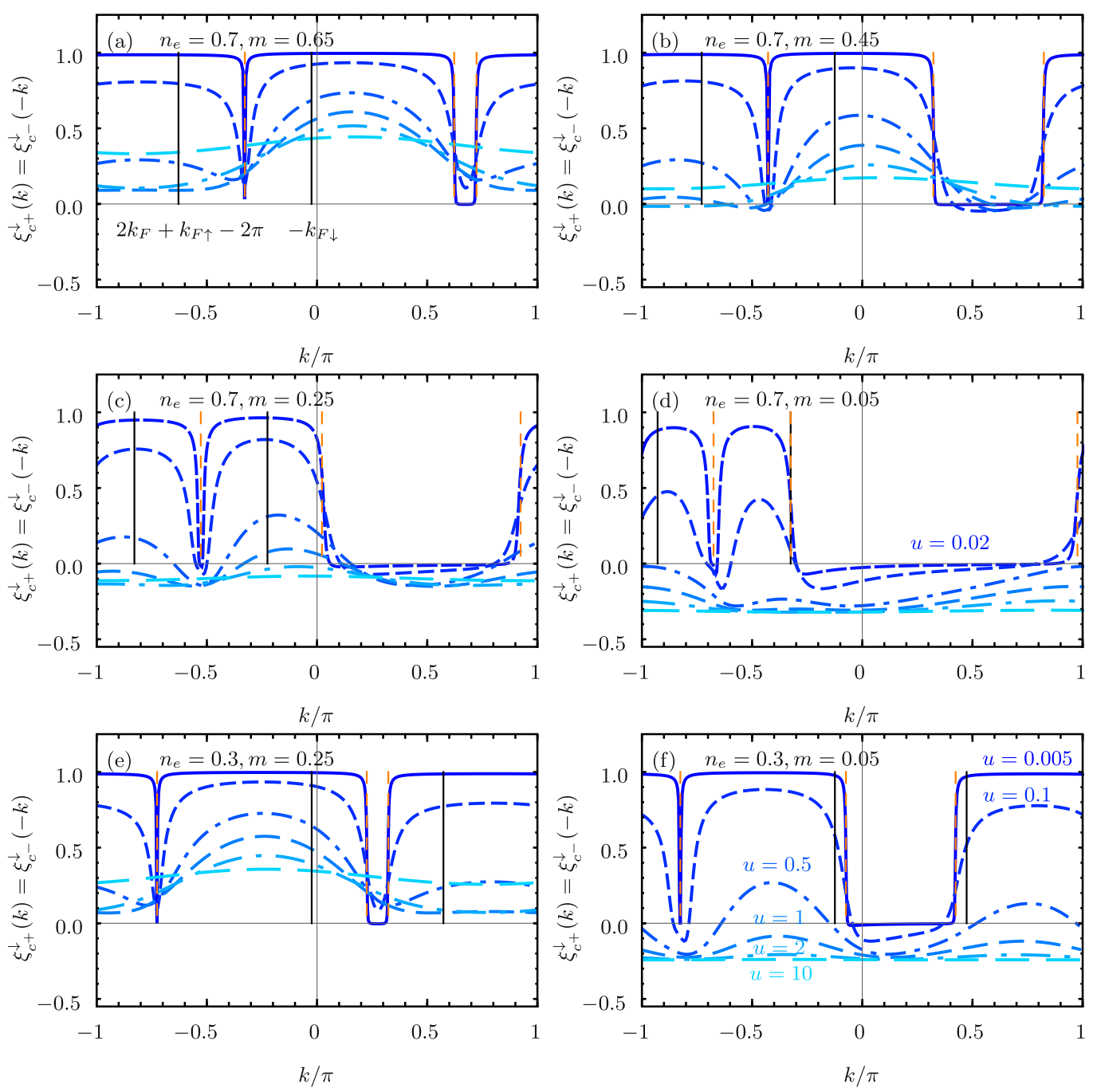

Fig. 27. The exponent $\xi_{c^{+}}^{\downarrow}(k)=\xi_{c^{-}}^{\downarrow}(-k)$ that controls the singularities in the vicinity of the $c^{+}$branch line of the $\sigma=\downarrow$ one-electron removal and LHB addition spectral function, Eq. (201) for $\sigma=\downarrow$ and $\gamma=-1$, as defined in Ref. [64], as a function of the momentum $k / \pi \in$ ] -1 , 1[ for several $u$ values, electronic density $n_{e}=0.7$, and spin densities (a) $m=0.65$, (b) $m=0.45$, (c) $m=0.25$, and (d) $m=0.05$, and for electronic density $n_{e}=0.3$ and spin densities (e) $m=0.25$ and (f) $m=0.05$.

Source: From Ref. [64].

Here $\hat{H}_{0}$ in whose expression $v$ is the particles Fermi velocity describes their kinetic energy near the BA Fermi points. The mobile impurity motion is described by $\hat{H}_{d}$. Furthermore, $\hat{H}_{\text {int }}$ contains the density-density interactions between the impurity and the particles in the vicinity of the Fermi points.

The operator $d^{\dagger}$ creates the mobile impurity with momentum near $k$ and energy near $\varepsilon_{1}(k)$. This is the excitation energy $\omega^{\tau}=c_{\tau} \varepsilon\left(q_{F}-k\right)$, Eq. (44). Its velocity is $\frac{\partial \varepsilon_{1}(k)}{\partial k} \frac{\partial}{\partial x}$. The quantities $\theta(x)$ and $\phi(x)$ in Eq. (202) are conventional bosonic fields. They satisfy the canonical commutation relation,

$$
\left[\phi(x), \nabla \theta\left(x^{\prime}\right)\right]=i \pi \delta\left(x-x^{\prime}\right) .
$$

The particles annihilation operator reads $\Psi_{B}(x, t) \approx e^{i \theta(x, t)}$. Moreover, $\nabla \frac{\theta(x)-\phi(x)}{2 \pi}$ and $-\nabla \frac{\theta(x)+\phi(x)}{2 \pi}$ are in the effective Hamiltonian $\hat{H}_{\text {int }}$ expression the densities of right and left movers, respectively. The interactions described by that effective Hamiltonian lead to the formation of low-energy particle-hole pairs. Those are crucial for the line shape of the dynamical correlation functions.

A key step of the MQIM method is that the interaction Hamiltonian term $\hat{H}_{\text {int }}$ can be removed by a unitary transformation, $\hat{U}^{\dagger}\left(\hat{H}_{0}+\hat{H}_{d}+\hat{H}_{\text {int }}\right) \hat{U}$, where [52,53],

$$
\hat{U}^{\dagger}=e^{i \int d x\left\{\frac{\delta_{+}}{2 \pi}[\tilde{\theta}(x)-\tilde{\phi}(x)]-\frac{\delta_{-}}{2 \pi}[\tilde{\theta}(x)+\tilde{\phi}(x)]\right\} d^{\dagger}(x) d(x)} \text { where } \delta_{ \pm}=2 \pi F_{B}\left( \pm Q \mid k_{0}\left(q_{F}-k\right)\right) .
$$



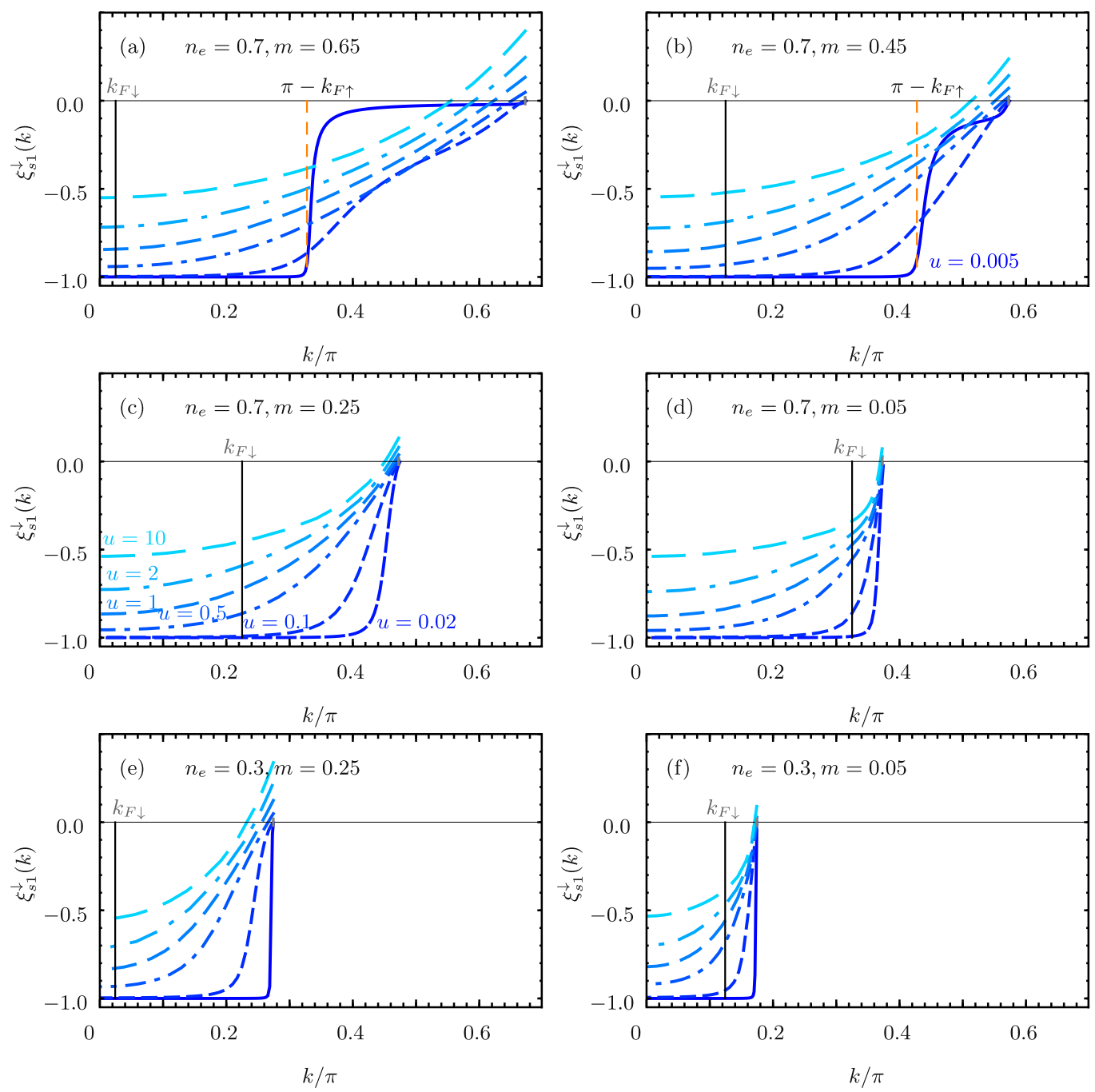

Fig. 28. The exponent $\xi_{s 1}^{\downarrow}(k)$ that controls the singularities in the vicinity of the $s 1$ branch line of the $\sigma=\downarrow$ one-electron removal and LHB addition spectral function, Eq. (201) for $\sigma=\downarrow$ and $\gamma=-1$, as defined in Ref. [64], as a function of the momentum $k / \pi \in$ ]0, 1[ for the same values of $u$, electronic density $n_{e}$, and spin density $m$ as in Fig. 27. (For $\left.k / \pi \in\right]-1,0\left[\right.$ the exponent $\xi_{s 1}^{\downarrow}(k)$ is given by $\xi_{s 1}^{\downarrow}(k)=\xi_{s 1}^{\downarrow}(-k)$ with $\left.-k / \pi \in\right] 0,1[$ as plotted here.) Source: From Ref. [64].

Here $\delta_{ \pm}$is a function defined in Eq. (15) of Ref. [57]. It is a particular case of the MQIM shift function $F_{B}\left(k \mid k^{\prime}\right)$ defined in Eqs. (7) and (8) of that reference. (As in Section 2.2, the MQIM shift-function $F_{B}(v \mid \mu)$ variables $v$ and $\mu$ of Ref. [57] are here denoted by $k$ and $k^{\prime}$, respectively, and the corresponding limiting values $\pm q$ by $\pm Q$, Eq. (11).)

We start by discussing the technical equivalence of the line shapes near the branch lines, as defined within the PDT and the MQIM. The relation between PDT and MQIM is addressed afterwards, from the point of view of the physical processes under consideration. A first issue to be clarified is the relation of the PDT $\tau=B, A, D$ exponents, Eq. (46), to those derived by the MQIM in Ref. [57]. In Appendix A it is rigorously shown that the following equalities exactly hold for the momentum range $k \in\left[0,2 \pi n_{b}\right]$ considered in that reference,

$$
\xi_{B}(k)=-\bar{\mu}_{-}(k), \quad \xi_{A}(k)=-\underline{\mu}_{+}(k) \text { and } \xi_{D}(k)=-\mu_{2}(k) .
$$

Such exact relations hold in spite of the apparent different expressions given in Eqs. (16)-(18) of Ref. [57] for the edge exponents on the right-hand side of the three equations in Eq. (205). The PDT exponent expressions,

$$
\xi_{\tau}(0)=\frac{\left(2 b_{\tau}-1\right)}{\xi^{1}}\left(1+\frac{\left(2 b_{\tau}-1\right)}{2 \xi^{1}}\right) \text { and } \xi_{\tau}(2 \pi n)=-2 \xi^{1}+2\left(\xi^{1}\right)^{2}+\frac{\left(2 b_{\tau}-1\right)}{\xi^{1}}\left(1+\frac{\left(2 b_{\tau}-1\right)}{2 \xi^{1}}\right),
$$


refer to the limiting momenta $k \rightarrow 0$ and $k \rightarrow 2 \pi n$, respectively. It follows from the equalities in Eq. (205) that they are fully equivalent to those provided for $\bar{\mu}_{-}(k)=-\xi_{B}(k), \mu_{+}(k)=-\xi_{A}(k)$, and $\mu_{2}(k)=-\xi_{D}(k)$ in Eqs. (19), (22), and (23) of Ref. [57] for $k \rightarrow 0$ and Eqs. (20), (24), and (25) of such a reference for $k \rightarrow 2 \pi n$.

On the one hand, the dynamical correlation functions exponents are within the PDT controlled by the pseudofermion phase shifts $2 \pi \Phi\left(q_{j}, q_{j^{\prime}}\right)=2 \pi \bar{\Phi}\left(k_{0}\left(\bar{q}_{j}\right), k_{0}\left(\bar{q}_{j^{\prime}}\right)\right)$, Eq. (12). They are thus also controlled by the related momentum rapidity phase shifts $2 \pi \bar{\Phi}\left(k, k^{\prime}\right)$, Eq. (13). Specifically, the edge singularities exponents are fully controlled by the pseudofermion phase shifts $2 \pi \Phi\left( \pm q_{F}, q^{\prime}\right)=2 \pi \bar{\Phi}\left( \pm Q, k_{0}\left(q^{\prime}\right)\right)$.

On the other hand, within the MQIM used in Ref. [57] to derive the same exponents, those are determined by the MQIM shift function $F_{B}\left(k \mid k^{\prime}\right)$. The two MQIM $\iota= \pm$ shift functions $F_{B}\left( \pm Q \mid k^{\prime}\right)$ play the major role, since they fully control the edge singularities exponents.

That the exact equalities in Eq. (205) hold for $k \in\left[0,2 \pi n_{b}\right]$ is an issue addressed in Appendix A. It is shown to result from the MQIM shift function $F_{B}\left(k \mid k^{\prime}\right)$ of Ref. [57] being exactly related to the momentum rapidity phase shift $\bar{\Phi}\left(k, k^{\prime}\right)$ in units of $2 \pi$, Eq. (13), as given in Eq. (A.8) of that Appendix. Hence the MQIM $\iota= \pm$ shift functions $F_{B}\left( \pm Q \mid k^{\prime}\right)=\delta_{ \pm} / 2 \pi$ that in Ref. [57] control the edge singularities exponents are simply related to the $\iota= \pm$ pseudofermion phase shifts $\Phi\left(\iota q_{F}, q^{\prime}\right)=\bar{\Phi}\left(\iota Q, k_{0}\left(q^{\prime}\right)\right)$ in units of $2 \pi$ as follows,

$$
F_{B}\left(\iota Q \mid k^{\prime}\right)=\frac{\delta_{\iota}}{2 \pi}=\frac{\xi^{1}}{2}-\bar{\Phi}\left(\iota Q, k^{\prime}\right)=\frac{\sqrt{K_{0}}}{2}-\bar{\Phi}\left(\iota Q, k^{\prime}\right) \text { for } \iota= \pm .
$$

Within the PDT, the $\iota= \pm$ pseudofermion phase shifts $2 \pi \Phi\left(\iota q_{F}, q^{\prime}\right)=2 \pi \bar{\Phi}\left(\iota Q, k_{0}\left(q^{\prime}\right)\right)$ control the important exponent functional, Eq. (37). In addition, they determine the parameter $\xi^{1}=1+\Phi\left(q_{F}, q_{F}\right)-\Phi\left(q_{F},-q_{F}\right)$, Eq. (18), that also appears in that exponent functional expression. This thus also applies the TLL parameter, $K_{0}=\left(\xi^{1}\right)^{2}=(1+$ $\left.\Phi\left(q_{F}, q_{F}\right)-\Phi\left(q_{F},-q_{F}\right)\right)^{2}$. The exact relation between the MIM shift functions $F_{B}\left(\iota Q \mid k^{\prime}\right)$ and the pseudofermion phase shifts $2 \pi \Phi\left(\iota q_{F}, q^{\prime}\right)=2 \pi \bar{\Phi}\left(\iota Q, k_{0}\left(q^{\prime}\right)\right)$ given in Eq. (207) clarifies the basic relation between the PDT and the MIM. (It plays the key role in the rigorous proof presented in Appendix A.)

The physical processes described by the MQIM are fully equivalent to those of the PDT that control the line shape of the dynamical correlation functions near the branch lines, Eqs. (196)-(195). Specifically:

(I) The unitary transformation, Eq. (204), that removes the interaction Hamiltonian term $\hat{H}_{\text {int }}$ is equivalent to the pseudoparticle-pseudofermion unitary transformation, $q_{j} \rightarrow q_{j}+2 \pi \Phi\left(q_{j}\right) / L$. Such a transformation removes the pseudoparticle energy spectrum interactions and introduces shake-up effects. Those result from the discrete canonical momentum value shifts $2 \pi \Phi\left(q_{j}\right) / L$, Eq. (33), under the transitions to the excited states. Such shake-up effects are behind a large number of small-momentum and low-energy pseudofermion-pseudofermion-hole processes (C). They occur in the linear part of the pseudofermion energy dispersions and lead to finite spectral-weight contributions. Similarly, in the case of the MQIM the interactions described by the effective Hamiltonian $\hat{H}_{\text {int }}$ lead to the formation of low-energy particle-hole pairs that are crucial for the line shape of the dynamical correlation functions.

(II) The mobile quantum impurity described by the effective Hamiltonian $\hat{H}_{d}$ in Eq. (202) corresponds to the pseudofermion or pseudofermion hole with canonical momentum away from the Fermi points. It is created within the PDT by processes (A). They occur under the transitions to excited states associated with the corresponding pseudofermion branch line.

(III) The effective Hamiltonian $\hat{H}_{0}$ in Eq. (202) describes the same processes near the Fermi points as the PDT processes of classes (B) and (C).

Hence although relying on apparently different physical starting points, the MQIM describes exactly the same processes as the PDT. This applies in the particular case of line shapes near the branch lines, as defined within the latter dynamical theory.

\section{General Outlook and future developments}

The static properties of the 1D Lieb-Liniger Bose gas, spin-1/2 isotropic Heisenberg chain, and 1D Hubbard model have been revisited in this review in terms of quantum liquids of pseudoparticles. The static quantities of these integrable systems are controlled by Landau parameters associated with the pseudoparticles residual interactions $f$ functions. The similarities to and differences from the usual Fermi liquid have been discussed.

The high-energy dynamical correlation functions of these integrable systems have been studied in the suitable and related pseudofermion representation of the BA solutions. The line shape in the vicinity of the high-energy one-particle spectral functions of the 1D Lieb-Liniger Bose gas and 1D Hubbard model is controlled by momentum dependent exponents. Such exponents have simple expressions in terms of the pseudofermions scattering phase shifts. For all models under review, the same applies to the line shape near the spectra edges of the two-particle dynamical correlation functions.

One of the goals of this review is to contribute to the further understanding of the fractionalized particles microscopic mechanisms that control the low- and high-energy properties of 1D correlated systems. In the case of the spin- $1 / 2 X X X$ chain, an exact expression of the spin currents of non-LWSs, Eq. (71), was used to study the elementary currents $j_{1}^{h}\left(q_{j}\right)$ in Eq. (72) that are conventionally associated with spinons. It has been found that the latter elementary currents describe the translational degrees of freedom of the model unpaired physical spins $1 / 2$ whose occupancies generate the energy eigenstates multiplet configurations. Our study includes an analysis of the exact elementary spin currents $j_{ \pm 1 / 2}$ in Eq. (72) carried by such physical 
spins $1 / 2$. It is found from it that one cannot associate the internal spin degrees of freedom of physical spins $1 / 2$ with the BA quantum numbers $q_{j}$ in the argument of the elementary current spectrum $j_{1}^{h}\left(q_{j}\right)$. Hence such internal spin degrees of freedom can neither be associated with the corresponding conventional spinons.

This reveals that spinons, as defined within integrable models, are not spin-1/2 particles. They do not contain the internal spin degrees of the physical spins whose translational degrees of freedom they describe. This clarification is important, because spinons are conventional fractionalized particles widely used in the description of the spin degrees of freedom of integrable systems. It applies to all integrable models with $S U(2)$ symmetries associated for instance with spin or $\eta$-spin degrees of freedom. However, conventional spinons describe correctly the translational degrees of freedom of the multiplets physical spins $1 / 2$. They thus can be successfully used in the description of some of the properties of $1 \mathrm{D}$ spin chains and electronic correlated models.

Moreover, the results under review confirm that the non-perturbative relation of the pseudoparticles to the models physical particles (bosons, spins $1 / 2$, and electrons) is more involved for models with more types of degrees of freedom and thus of increasing complexity. Specifically, it becomes more involved as one goes from the Abelian global $U(1)$ symmetry $1 \mathrm{D}$ Lieb-Liniger Bose gas, to the non-Abelian global $S U(2)$ symmetry spin-1/2 XXX chain, and further to the much more involved non-Abelian global $[S U(2) \otimes S U(2) \otimes U(1)] / Z_{2}^{2}$ symmetry 1D Hubbard model. The discussion of the increase in complexity and of the role played in it by the interplay of the BA pseudoparticles with the global symmetries representations, has unified the results being reviewed. It thus contributed to the further understanding of their physical meaning.

Concerning future studies in this field, an interesting future development would be to extend the computations of the PDT high-energy dynamical correlation functions beyond the vicinity of the one- and two-particle spectra edges and oneparticle singular branch and border lines at finite $u$. The derivation of accurate finite- $u$ line shapes over the whole $(k, \omega)$ plane, as those obtained in Refs. [156-158] for the $u \rightarrow \infty$ limit, would require more demanding numerical computations than those employed in these references. Future developments and improvements in numerical techniques may allow such computations of the finite- $u$ one- and two-electron spectral-weight distributions.

A development that also deserves future studies, refers to the need of accounting for electron finite-range and long-range interactions beyond the 1D Hubbard model in the description of the microscopic mechanisms behind the ARPES in 1D and quasi-1D metallic states of actual physical systems. Indeed, within that integrable model the parameter $\xi_{0}$ and related charge TLL parameter $K_{\rho}^{0} \equiv \xi_{0}^{2} / 2[3,4,272]$ can vary in the intervals $\xi_{0} \in[1, \sqrt{2}]$ and $K_{\rho}^{0} \in[1 / 2,1]$, respectively. (The $m \rightarrow 0$ parameter $\xi_{0}$ is related to phase shifts, as given Eq. (H.13) of Appendix H.) Through Eq. (164), such intervals correspond to a suppression of density of states exponent range $\alpha_{0} \in[0,1 / 8]$.

In contrast, the density of states suppression exponent $\alpha$ experimentally measured for instance in 1D metallic states of $\mathrm{Bi} / \mathrm{InSb}(001)$ [25], 1D line defects in transition dichalcogenides such as $\mathrm{MoSe}_{2}$ [26], and quasi-1D conductors [272] belongs typically to the interval $\alpha \in[0.50,0.80]$. This implies that for such systems the charge TLL parameter $K_{\rho}$ and the related parameter $\xi_{0}=\sqrt{2 K_{\rho}}$ have values $K_{\rho}<1 / 2$ and $\tilde{\xi}_{0}<1$, respectively. As discussed in Ref. [3], such values result from electron interactions beyond onsite, i.e. finite-range interactions (of at least one lattice spacing) or long-range interactions that must be accounted for. (Here $K_{\rho}$ and $\tilde{\xi}_{0}$ is our notation for the parameters corresponding to the 1D Hubbard model parameters $K_{\rho}^{0} \in[1 / 2,1]$ and $\xi_{0} \in[1, \sqrt{2}]$ in the general case of models with electron finite-range or long-range interactions for which they have values within the extended intervals $K_{\rho} \in[1 / 8,1]$ and $\tilde{\xi}_{0} \in[1 / 2, \sqrt{2}]$, respectively.)

The electron finite-range renormalized theory introduced in Ref. [26] involves the transformation of the 1D Hubbard model into non-integrable models. The $c$ pseudofermions are not well defined in such models except at and near the $c$ band Fermi points. The transformation involves gently turning on the finite-range part of electronic potentials $V_{\mathrm{el}}(r)$. This leads to a renormalized effective potential between the $c$ pseudofermions at and near the $c$ band Fermi points and the $c$-band hole created in that band under one-electron removal excitations. Within the MQIM, that $c$-band hole refers to the mobile quantum impurity. The corresponding interaction between such $c$ pseudofermions and the emerging $c$ band hole associated with their renormalized potential has an effective range equal or larger than one lattice spacing. The effective range plays an important role for instance in scattering of atoms [273].

The renormalized theory of Ref. [26] is applied in that reference to the metallic 1D line defects in MoSe $\mathrm{S}_{2}$ for which the effects of the $c$ pseudofermions and $c$ band-hole effective range are small. Specifically, the effects of such an effective range give rise to small changes in the spectral-function momentum dependent exponents that control the spectral peaks distribution that lay within the ARPES experimental uncertainty. Therefore, they have been neglected in the studies of Ref. [26]. An interesting development is to account for such interaction effective-range effects within the $c$ pseudofermions phase-shift renormalization. Indeed, this is needed in the case of the description of larger finite-range or long-range microscopic mechanisms behind the ARPES of other 1D metallic states as those in 1D Bi/InSb(001) [25] and quasi-1D conductors [13-16,272].

Another interesting future development would involve further advances in the use of ultra-cold atoms in optical lattices to simulate the 1D correlated models under review here and related models [28,30,33-38]. This would provide complementary information on both the spectral-functions line shapes over the whole $(k, \omega)$ plane and the exotic fractionalized particles and related composite particles reviewed in this paper.

Furthermore, spectral signatures of fractionalized particles have been clearly seen in quantum wires [39]. This makes them potential candidates for technological applications in quantum computers. As mentioned in Section 1, the further understanding the properties of the fractionalized particles may be important for such quantum technologies. It may as well 
as helping to develop more complete theories of superconductivity and conduction in low-dimensional condensed-matter systems.

Whether fractionalized particles and their composite particles also emerge in two-dimensional correlated problems is an open problem of high scientific interest. The global $[S U(2) \otimes S U(2) \otimes U(1)] / Z_{2}^{2}$ symmetry found in Ref. [147] applies to the Hubbard model on any bipartite lattice. Hence rotated-electron representations associated with the model energy eigenstates, as those reviewed in Section 4.3 for the 1D lattice, apply as well to the model on the square and other bipartite lattices. Whether the rotated-electron degrees of freedom separation reported in this paper partially survives for the Hubbard model on the square lattice or other low-dimensional bipartite lattices is a problem of physical interest that deserves further investigations.

A possible scenario is that for the Hubbard model on the square lattice fractionalized particles and/or their composite particles emerge from a rotated-electron degrees of freedom separation. However, their interactions would not be of the simple zero-momentum forward-scattering type. This is a property specific to the pseudofermions of integrable 1D models. It results from the occurrence of an infinite number of conservation laws [250,251,274], which is associated with their integrability. The Hubbard model on the square lattice is not integrable. Nonetheless, a scenario within which there are energy and momentum exchanges among the charge-like and spin-like fractionalized particles and/or their composite particles is a possible interesting future development.

The interactions of such elementary fractionalized particles and their possible composite particles could be a simpler problem to handle than that of the underlying many-electron interactions. This research direction could be of interest for developing a further understanding of the over 30 years old unsolved problem of the microscopic mechanisms behind the cuprates superconductivity [275-279] and its relation to the properties of the undoped Mott-Hubbard insulators parent compounds [280,281].

\section{Acknowledgments}

We thank M. A. N. Araújo, D. Baeriswyl, P.-A. Bares, D. Bozi, D. K. Campbell, A. H. Castro Neto, T. Čadež, R. G. Dias, J. M. E. Guerra, F. Guinea, P. Horsch, H. Q. Lin, A. Luther, L. M. Martelo, A. Moreno, S. Östlund, K. Penc, R. G. Pereira, N. M. R. Peres, T. Prosen, J. M. Román, M. J. Sampaio, and J. M. P. L. Santos for illuminating discussions and their contributions to common collaborations that led to some of the results on the issues being reviewed. We also thank N. Andrei, E. Castro, and $\mathrm{H}$. Johannesson for illuminating discussions, and M. Belsley for the critical reading of a preliminary version of the review manuscript and useful discussions. We acknowledge our former collaborator, the late S.-J. Gu, for his important contributions to the success of our common research related to the topics reviewed here. Over the long course of his study of this problem, J. M. P. C. has benefited from discussions with P. W. Anderson, M. C. Asensio, M. Batzill, L. Carlos, Y.-H. Chen, R. Claessen, F. Essler, J. Ferrer, X.-W. Guan, E. Jeckelmann, S.-i. Kimura, V. E. Korepin, P. A. Lee, R. Micnas, S. Nemati, Y. Ohtsubo, T. Ribeiro, A. W. Sandvik, M. Sing, A. L. L. Videira, J. Voit, X.-G. Wen, S. R. White, and X. Zotos. He especially wishes to acknowledge his former collaborators, the late K. Maki, A. Muramatsu, and A. A. Ovchinnikov, for illuminating discussions on 1D correlated systems and their contributions to his understanding of the Hubbard model. He also acknowledges the late A. Imambekov for discussions that were helpful in writing this review. P. D. S. thanks K. -J. -B. Lee, J. W. Rasul, and P. Schlottmann for discussions on integrable systems. We thank the FEDER through the COMPETE Program and the Portuguese FCT in the framework of the Strategic Projects UID/FIS/04650/2013 and UID/CTM/04540/2013 and the support of the Beijing Computational Science Research Center where part of this review was written.

\section{Appendix A. Equality of the Bose gas edge singularities exponents as derived by the PDT and MIM}

The goals of this Appendix are to obtain the 1D Bose gas pseudofermion phase shift relation to the MQIM shift function $\Phi\left(\iota q_{F}, q_{j^{\prime}}\right)=\frac{\xi^{1}}{2}-F_{B}\left(\iota k_{0}\left(q_{F}\right), k_{0}\left(q_{j^{\prime}}\right)\right)$ used to derive expression, Eq. (39), and to provide a rigorous proof of the equalities given in Eq. (205). As a first step to reach such goals, the relation provided in Eq. (207) is derived. As in Sections 2.2 and 7.4, here we denote the MQIM shift function $F_{B}(v \mid \mu)$ variables $v$ and $\mu$ of Ref. [57] by $k$ and $k^{\prime}$, respectively. The corresponding limiting values $\pm q$ are denoted by $\pm Q$, Eq. (11). Moreover, as elsewhere in this paper the Tomonaga-Luttinger liquid parameter is denoted by $K_{0}$. (In Ref. [57] it is denoted by $K$.)

The MQIM shift function $F_{B}\left(k \mid k^{\prime}\right)$ has been defined in Ref. [57] as the solution of the integral equation,

$$
F_{B}\left(k \mid k^{\prime}\right)=\frac{1}{2}-\frac{1}{\pi} \arctan \left(\frac{k-k^{\prime}}{c}\right)+\frac{1}{\pi c} \int_{-Q}^{Q} d k^{\prime \prime} \frac{F_{B}\left(k \mid k^{\prime}\right)}{1+\left(\frac{k-k^{\prime \prime}}{c}\right)^{2}} .
$$

The momentum rapidity phase shift $\bar{\Phi}\left(k, k^{\prime}\right)$ in units of $2 \pi$ and the MQIM shift function $F_{B}\left(k \mid k^{\prime}\right)$ obey integral equations, Eqs. (13) and (A.1), respectively, with the same kernel. Hence their sum $\bar{\Phi}\left(k, k^{\prime}\right)+F_{B}\left(k \mid k^{\prime}\right)$ obeys an integral equation with such a kernel and whose free term is merely the sum of those of Eqs. (13) and (A.1). This gives,

$$
\bar{\Phi}\left(k, k^{\prime}\right)+F_{B}\left(k \mid k^{\prime}\right)=\frac{1}{2}+\frac{1}{\pi c} \int_{-Q}^{Q} d k^{\prime \prime} \frac{\bar{\Phi}\left(k, k^{\prime}\right)+F_{B}\left(k \mid k^{\prime}\right)}{1+\left(\frac{k-k^{\prime \prime}}{c}\right)^{2}} .
$$


Next we show that the function $\bar{\Phi}\left(k, k^{\prime}\right)+F_{B}\left(k \mid k^{\prime}\right)$ does not depend on $k^{\prime}$. Indeed, one finds from the use of Eq. (A.2) that the derivative $\partial\left(\bar{\Phi}\left(k, k^{\prime}\right)+F_{B}\left(k \mid k^{\prime}\right)\right) / \partial k^{\prime}$ obeys the equation,

$$
\frac{\partial\left(\bar{\Phi}\left(k, k^{\prime}\right)+F_{B}\left(k \mid k^{\prime}\right)\right)}{\partial k^{\prime}}=\frac{1}{\pi c} \int_{-Q}^{Q} d k^{\prime \prime} \frac{\frac{\partial\left(\bar{\Phi}\left(k, k^{\prime}\right)+F_{B}\left(k \mid k^{\prime}\right)\right)}{\partial k^{\prime}}}{1+\left(\frac{k-k^{\prime \prime}}{c}\right)^{2}} .
$$

Since the free term of this equation vanishes, it follows from known properties of this type of integral equations that its unique solution is $\partial\left(\bar{\Phi}\left(k, k^{\prime}\right)+F_{B}\left(k \mid k^{\prime}\right)\right) / \partial k^{\prime}=0$. This confirms that $\alpha(k) \equiv \bar{\Phi}\left(k, k^{\prime}\right)+F_{B}\left(k \mid k^{\prime}\right)$ is an even function, $\alpha(k)=\alpha(-k)$, of only the variable $k$. As given in Eq. (A.2), it obeys the integral equation,

$$
\alpha(k)=\frac{1}{2}+\frac{1}{\pi c} \int_{-Q}^{Q} d k^{\prime} \frac{\alpha\left(k^{\prime}\right)}{1+\left(\frac{k-k^{\prime}}{c}\right)^{2}} .
$$

One finds from simple manipulations of the integral equation, Eq. (13), obeyed by the momentum rapidity phase shift $\bar{\Phi}\left(k, k^{\prime}\right)$ in units of $2 \pi$ that the function,

$$
\xi^{1}(k)=1+\bar{\Phi}(k, Q)-\bar{\Phi}(k,-Q),
$$

is the unique solution of the integral equation,

$$
\xi^{1}(k)=1+\frac{1}{\pi c} \int_{-Q}^{Q} d k^{\prime} \frac{\xi^{1}\left(k^{\prime}\right)}{1+\left(\frac{k-k^{\prime}}{c}\right)^{2}} .
$$

Since it is an even function, $\xi^{1}(k)=\xi^{1}(-k)$, one finds that $\xi^{1}=\sqrt{K_{0}}=\xi^{1}(\iota Q)$ where $\xi^{1}=1+\Phi\left(q_{F}, q_{F}\right)-\Phi\left(q_{F},-q_{F}\right)$ is the phase-shift parameter, Eq. (18). It is related to the TLL parameter as $K_{0}=\left(\xi^{1}\right)^{2}=\left(1+\Phi\left(q_{F}, q_{F}\right)-\Phi\left(q_{F},-q_{F}\right)\right)^{2}$.

The functions $\alpha(k)$ and $\xi^{1}(k)$ obey again integral equations, Eqs. (A.4) and (A.6), respectively, with the same kernel. Hence the difference function $\xi^{1}(k)-\alpha(k)$ obeys an integral equation with that kernel and whose free term is the difference of those of Eqs. (A.6) and (A.4). The latter free term reads 1/2. It follows that the difference function $\xi^{1}(k)-\alpha(k)$ obeys the same integral equation, Eq. (A.4), as the function $\alpha(k)$. Since the solution of that integral equation is unique, one arrives to the exact relations,

$$
\alpha(k)=\frac{\xi^{1}(k)}{2} \text { and } \alpha(\iota Q)=\frac{\xi^{1}}{2} .
$$

By combining these relations with the expressions $\alpha(k) \equiv \bar{\Phi}\left(k, k^{\prime}\right)+F_{B}\left(k \mid k^{\prime}\right)$ and $\alpha(\iota Q) \equiv \bar{\Phi}\left(\iota Q, k^{\prime}\right)+F_{B}\left(\iota Q \mid k^{\prime}\right)$, one readily finds that,

$$
F_{B}\left(k \mid k^{\prime}\right)=\frac{1}{2}[1+\bar{\Phi}(k, Q)-\bar{\Phi}(k,-Q)]-\bar{\Phi}\left(k, k^{\prime}\right),
$$

and thus $F_{B}\left(\iota Q \mid k^{\prime}\right)=\xi^{1} / 2-\bar{\Phi}\left(\iota Q, k^{\prime}\right)$. By accounting for the $\xi^{1}(k)$ expression, Eq. (A.5), the latter is indeed the expression, Eq. (207).

The important PDT $\iota= \pm c$ pseudofermion Fermi points fluctuations functionals, Eq. (37), can be expressed in terms of the MQIM shift function $F_{B}\left(k \mid k^{\prime}\right)$ as given in Eq. (39). To reach that expression, one uses the relation $F_{B}\left(\iota Q \mid k^{\prime}\right)=\xi^{1} / 2-\bar{\Phi}\left(\iota Q, k^{\prime}\right)$ that can be written as $\bar{\Phi}\left(\iota Q, k^{\prime}\right)=\xi^{1} / 2-F_{B}\left(\iota Q \mid k^{\prime}\right)$ so that,

$$
\Phi\left(\iota q_{F}, q_{j^{\prime}}\right)=\frac{\xi^{1}}{2}-F_{B}\left(\iota k_{0}\left(q_{F}\right) \mid k_{0}\left(q_{j^{\prime}}\right)\right) .
$$

Here $k_{0}\left(q_{j^{\prime}}\right)$ is the ground-state momentum rapidity function. The use of this relation in Eq. (37) readily leads to the functional dimensions expression, Eq. (39).

It follows from Eq. (207) that the $\tau=B, A, D$ exponents in Eq. (46) can be expressed in terms of the $\iota= \pm$ shift functions $F_{B}\left(\iota Q \mid k^{\prime}\right)$ as follows,

$$
\xi_{\tau}(k)=-1+\sum_{\iota= \pm}\left(\frac{\xi^{1}}{2}+\iota \frac{b_{\tau}}{\xi^{1}}-\bar{\Phi}\left(\iota Q, k_{0}\left(q_{F}-k\right)\right)\right)^{2}=-1+\sum_{\iota= \pm}\left(F_{B}\left(\iota Q \mid k_{0}\left(q_{F}-k\right)\right)+\iota \frac{b_{\tau}}{\xi^{1}}\right)^{2} .
$$

The function $k_{0}\left(q_{F}-k\right)$ in this expression is the ground-state momentum rapidity function $k_{0}(q)$ at $q=q_{F}-k$. (Such a function is the solution of the ground-state BA equation.)

It is useful to express the second expression in Eq. (A.10) in terms of the functions $\delta_{ \pm}$in Eq. (204), defined in Eq. (15) of Ref. [57]. This gives,

$$
\begin{aligned}
\xi_{\tau}(k) & =-1+\left(\frac{\delta_{+}}{2 \pi}+\frac{b_{\tau}}{\xi^{1}}\right)^{2}+\left(\frac{\delta_{-}}{2 \pi}-\frac{b_{\tau}}{\xi^{1}}\right)^{2}=-1+\frac{\delta_{+}^{2}+\delta_{-}^{2}}{(2 \pi)^{2}}+\frac{b_{\tau}\left(\delta_{+}-\delta_{-}\right)}{2 \pi \xi^{1}}+\frac{2 b_{\tau}^{2}}{\left(\xi^{1}\right)^{2}} \\
& =-1+\frac{\delta_{+}^{2}+\delta_{-}^{2}}{(2 \pi)^{2}}+\frac{b_{\tau}\left(\delta_{+}-\delta_{-}\right)}{2 \pi \sqrt{K_{0}}}+\frac{2 b_{\tau}^{2}}{K_{0}} .
\end{aligned}
$$


Or specifically for each of the three dynamical correlation functions under consideration,

$$
\begin{aligned}
& \xi_{B}(k)=-1+\frac{\delta_{+}^{2}+\delta_{-}^{2}}{(2 \pi)^{2}} \text { and } \xi_{A}(k)=-1+\frac{\delta_{+}^{2}+\delta_{-}^{2}}{(2 \pi)^{2}}+\frac{\delta_{+}-\delta_{-}}{2 \pi \sqrt{K_{0}}}+\frac{2}{K_{0}}, \\
& \xi_{D}(k)=-1+\frac{\delta_{+}^{2}+\delta_{-}^{2}}{(2 \pi)^{2}}+\frac{\delta_{+}-\delta_{-}}{4 \pi \sqrt{K_{0}}}+\frac{1}{2 K_{0}},
\end{aligned}
$$

where we used that $b_{B}=0, b_{A}=1$, and $b_{D}=1 / 2$.

The exponents $-\bar{\mu}_{-},-\underline{\mu}_{+}$, and $-\mu_{2}$ in Eq. (205) were found in Ref. [57] to read,

$$
\begin{aligned}
& -\bar{\mu}_{-}=-1+\frac{1}{2}\left(\frac{\delta_{+}-\delta_{-}}{2 \pi}\right)^{2}+\frac{1}{2}\left(\frac{\delta_{+}+\delta_{-}}{2 \pi}\right)^{2}, \\
& -\underline{\mu}_{+}=-1+\frac{1}{2}\left(\frac{2}{\sqrt{K_{0}}}+\frac{\delta_{+}-\delta_{-}}{2 \pi}\right)^{2}+\frac{1}{2}\left(\frac{\delta_{+}+\delta_{-}}{2 \pi}\right)^{2}, \\
& -\mu_{2}=-1+\frac{1}{2}\left(\frac{1}{\sqrt{K_{0}}}+\frac{\delta_{+}-\delta_{-}}{2 \pi}\right)^{2}+\frac{1}{2}\left(\frac{\delta_{+}+\delta_{-}}{2 \pi}\right)^{2},
\end{aligned}
$$

as given in Eqs. (16)-(18) of that reference. It is a simple exercise to show that these expressions can be rewritten as,

$$
\begin{aligned}
& -\bar{\mu}_{-}=-1+\frac{\delta_{+}^{2}+\delta_{-}^{2}}{(2 \pi)^{2}} ; \quad-\underline{\mu}_{+}=-1+\frac{\delta_{+}^{2}+\delta_{-}^{2}}{(2 \pi)^{2}}+\frac{\delta_{+}-\delta_{-}}{2 \pi \sqrt{K_{0}}}+\frac{2}{K_{0}}, \\
& -\mu_{2}=-1+\frac{\delta_{+}^{2}+\delta_{-}^{2}}{(2 \pi)^{2}}+\frac{\delta_{+}-\delta_{-}}{4 \pi \sqrt{K_{0}}}+\frac{1}{2 K_{0}},
\end{aligned}
$$

respectively.

Finally, comparison of the expressions provided in Eqs. (A.12) and (A.14) confirms the validity of the equalities given in Eq. (205).

\section{Appendix B. Some additional results on the spin-1/2 XXX chain and 1D Hubbard model TBA solutions} by,

The function $\Theta_{n n^{\prime}}(x)$ appearing in Eq. (58) for the spin-1/2 XXX chain and in Eq. (129) for the 1D Hubbard model is given

$$
\begin{aligned}
\Theta_{n n^{\prime}}(x) & =\delta_{n, n^{\prime}}\left\{2 \arctan \left(\frac{x}{2 n}\right)+\sum_{l=1}^{n-1} 4 \arctan \left(\frac{x}{2 l}\right)\right\} \\
& +\left(1-\delta_{n, n^{\prime}}\right)\left\{2 \arctan \left(\frac{x}{\left|n-n^{\prime}\right|}\right)+2 \arctan \left(\frac{x}{n+n^{\prime}}\right)+\sum_{l=1}^{\frac{n+n^{\prime}-\left|n-n^{\prime}\right|}{2}-1} 4 \arctan \left(\frac{x}{\left|n-n^{\prime}\right|+2 l}\right)\right\},
\end{aligned}
$$

where $n, n^{\prime}=1, \ldots, \infty$ and $\delta_{n, n^{\prime}}$ is the usual Kronecker symbol. Its derivative reads,

$$
\begin{aligned}
\Theta_{n n^{\prime}}^{[1]}(x) & =\frac{d \Theta_{n, n^{\prime}}(x)}{d x}=\delta_{n, n^{\prime}}\left\{\frac{1}{n\left(1+\left(\frac{x}{2 n}\right)^{2}\right)}+\sum_{l=1}^{n-1} \frac{2}{l\left(1+\left(\frac{x}{2 l}\right)^{2}\right)}\right\}+\left(1-\delta_{n, n^{\prime}}\right)\left\{\frac{2}{\left|n-n^{\prime}\right|\left(1+\left(\frac{x}{\left|n-n^{\prime}\right|}\right)^{2}\right)}\right. \\
& \left.+\sum_{l=1}^{\frac{n+n^{\prime}-\left|n-n^{\prime}\right|-2}{2}} \frac{4}{\left(\left|n-n^{\prime}\right|+2 l\right)\left(1+\left(\frac{x}{\left|n-n^{\prime}\right|+2 l}\right)^{2}\right)}+\frac{2}{\left(n+n^{\prime}\right)\left(1+\left(\frac{x}{n+n^{\prime}}\right)^{2}\right)}\right\} .
\end{aligned}
$$

The BA momentum bands have often exotic limiting values, in some cases dependent on the densities. In the case of the spin-1/2XXX chain, each $n$-band, such that $q_{j+1}-q_{j}=2 \pi / L$, has a momentum range $q_{j} \in\left[q_{n}^{-}, q_{n}^{+}\right]$whose limiting momentum values $q_{n}^{ \pm}$are given in Eq. (59). Within the TL they read,

$$
q_{n}^{ \pm}= \pm \frac{\pi}{L}\left(L_{n}-1\right) \approx \pm \pi m_{n} \text { where } m_{n}=n_{n}+n_{n}^{h}=L_{n} / L \text { and } n_{n}^{h}=N_{n}^{h} / L .
$$

The 1D Hubbard model set $j=1, \ldots, L_{\beta}$ of $\beta=c, \alpha n$ bands discrete momentum values $q_{j}$ belong to well-defined domains, $q_{j} \in\left[q_{\beta}^{-}, q_{\beta}^{+}\right]$, where

$$
\begin{aligned}
q_{c}^{ \pm} & = \pm \frac{\pi}{L}(L-1) \approx \pm \pi \text { for } N^{S U(2)} \text { odd } ; q_{c}^{ \pm}= \pm \frac{\pi}{L}(L-1 \pm 1) \approx \pm \pi \text { for } N^{S U(2)} \text { even }, \\
q_{\alpha n}^{ \pm} & = \pm \frac{\pi}{L}\left(L_{\alpha n}-1\right),
\end{aligned}
$$

and the number $N^{S U(2)}$ is given in Eq. (133). 
Finally, the 1D Hubbard model energy eigenvalues and related energy scales are provided. The former have the following general functional form when expressed in terms of the $\beta=c, \alpha$ band momentum distribution functions $N_{\beta}\left(q_{j}\right)$ and number $M_{\alpha,-1 / 2}$ of unpaired spins $(\alpha=s)$ and unpaired $\eta$-spins $(\alpha=\eta)$ of projection $-1 / 2$,

$$
E=\sum_{j=1}^{L}\left(N_{c}\left(q_{j}\right) E_{c}\left(q_{j}\right)+U / 4-\mu_{\eta}\right)+\sum_{\alpha=\eta, s} \sum_{n=1}^{\infty} \sum_{j=1}^{L_{\alpha n}} N_{\alpha n}\left(q_{j}\right) E_{\alpha n}\left(q_{j}\right)+\sum_{\alpha=\eta, s} 2 \mu_{\alpha} M_{\alpha,-1 / 2} .
$$

Here,

$$
2 \mu_{s}=2 \mu_{B}|H| ; \quad 2 \mu_{\eta}=2|\mu| \text { for } n_{e} \neq 1 ; \quad 2 \mu_{\eta}=2 \mu_{0} \text { for } n_{e}=1,
$$

and

$$
\begin{aligned}
E_{c}\left(q_{j}\right) & =-2 t \cos k^{c}\left(q_{j}\right)-U / 2+\mu_{\eta}-\mu_{s}, \\
E_{\alpha n}\left(q_{j}\right) & =2 n \mu_{\alpha}+\delta_{\alpha, \eta}\left(2 t \sum_{\iota= \pm 1} \sqrt{1-\left(\Lambda^{\eta n}\left(q_{j}\right)-i \iota n u\right)^{2}}-n U\right) \text { where } \alpha=\eta, s \text { and } n=1, \ldots, \infty .
\end{aligned}
$$

For each $u>0$ energy and momentum eigenstate, the momentum rapidity function $k^{c}\left(q_{j}\right)$, related $c$-band rapidity $\Lambda^{c}\left(q_{j}\right)=$ [sin $\left.k^{c}\left(q_{j}\right)\right] / u$, and the $n=1, \ldots, \infty$ rapidity functions $\Lambda^{\eta n}\left(q_{j}\right)$ are the solutions of the TBA equations, Eqs. (128) and (129).

The energy scale $2 \mu^{0}$ in Eq. (B.6) is the $n_{e}=1$ half-filling Mott-Hubbard gap [69-71]. For $u>0$ it is an even function of the spin density $m$ that remains finite for the whole interval, $m \in[-1,1]$. For instance, at spin densities $m=0[69,70]$ and $m=-1,1$ it reads,

$$
\begin{aligned}
2 \mu^{0} & =U-4 t+8 t \int_{0}^{\infty} d \omega \frac{J_{1}(\omega)}{\omega\left(1+e^{2 \omega u}\right)}=\frac{16 t^{2}}{U} \int_{1}^{\infty} d \omega \frac{\sqrt{\omega^{2}-1}}{\sinh \left(\frac{2 \pi t \omega}{U}\right)} \text { for } m=0, \\
& =\sqrt{(4 t)^{2}+U^{2}}-4 t \text { for } m=-1,1,
\end{aligned}
$$

respectively. Its $u \ll 1$ limiting behaviors [71] are $2 \mu^{0} \approx(8 / \pi) \sqrt{t U} e^{-2 \pi\left(\frac{t}{U}\right)}$ at $m=0$ and $2 \mu^{0} \approx U^{2} / 8 t$ for $m= \pm 1$. Its $u \gg 1$ behavior is $2 \mu^{0} \approx(U-4 t)$ for the whole $m \in[-1,1]$ range.

For electronic densities $\left.n_{e} \in\right] 0,1[$, spin density $m=0$, and the whole $u>0$ range the maximum sn pseudoparticle pairing energy in Eq. (184) vanishes. The maximum $\eta n$ pseudoparticle pairing energy in that equation has for such densities and limiting interaction values $u \rightarrow 0$ and $u \gg 1$ the following limiting behaviors,

$$
\begin{aligned}
W_{\eta n}^{\text {pair }} & =\left|\varepsilon_{\eta n}^{0}(0)\right|=4 t \cos \left(\frac{\pi}{2} n_{e}\right)=2|\mu| \text { for } u \rightarrow 0, \\
& =\frac{8\left(1-n_{e}\right) t^{2}}{n U}\left(1-\frac{\sin \left(2 \pi\left(1-n_{e}\right)\right)}{2 \pi\left(1-n_{e}\right)}\right) \text { for } u \gg 1,
\end{aligned}
$$

respectively.

For the electronic density interval $\left.n_{e} \in\right] 0,1\left[\right.$ and spin density $m \rightarrow n_{e}$, the maximum $s n$ and $\eta n$ pseudoparticle pairing energies in Eq. (184) have the following analytical expressions valid for the whole $u>0$ range,

$$
\begin{aligned}
W_{s n}^{\text {pair }} & =\left|\varepsilon_{s n}^{0}(0)\right|=\sqrt{(4 t)^{2}+(n U)^{2}} \frac{1}{\pi} \arctan \left(\frac{\sqrt{(4 t)^{2}+(n U)^{2}}}{(n U)} \tan \left(\pi n_{e}\right)\right) \\
& \left.-n U n_{e}-\frac{4 t}{\pi} \cos \left(\pi n_{e}\right) \arctan \left(\frac{4 t \sin \left(\pi n_{e}\right)}{n U}\right) \text { for } n_{e} \in\right] 0,1\left[\text { and } m \rightarrow n_{e},\right.
\end{aligned}
$$

and

$$
\begin{aligned}
W_{\eta n}^{\text {pair }} & =\left|\varepsilon_{\eta n}^{0}(0)\right|=\sqrt{(4 t)^{2}+(n U)^{2}} \frac{1}{\pi} \arctan \left(\frac{\sqrt{(4 t)^{2}+(n U)^{2}}}{(n U)} \tan \left(\pi\left(1-n_{e}\right)\right)\right) \\
& \left.-n U\left(1-n_{e}\right)-\frac{4 t}{\pi} \cos \left(\pi\left(1-n_{e}\right)\right) \arctan \left(\frac{4 t \sin \left(\pi\left(1-n_{e}\right)\right)}{n U}\right) \text { for } n_{e} \in\right] 0,1\left[\text { and } m \rightarrow n_{e},\right.
\end{aligned}
$$

respectively. For $u \rightarrow 0$ and $u \gg 1$ these expressions simplify to,

$$
\begin{aligned}
W_{s n}^{\text {pair }} & =\left|\varepsilon_{s n}^{0}(0)\right|=4 t \sin ^{2}\left(\frac{\pi n_{e}}{2}\right)=2 \mu_{B} H_{c} \text { for } u \rightarrow 0, \\
& =\frac{8 n_{e} t^{2}}{n U}\left(1-\frac{\sin \left(2 \pi n_{e}\right)}{2 \pi n_{e}}\right)=\frac{1}{n} 2 \mu_{B} H_{c} \text { for } u \gg 1,
\end{aligned}
$$

and

$$
W_{\eta n}^{\text {pair }}=\left|\varepsilon_{\eta n}^{0}(0)\right|=4 t \sin ^{2}\left(\frac{\pi\left(1-n_{e}\right)}{2}\right)=2|\mu| \text { for } u \rightarrow 0,
$$




$$
=\frac{8\left(1-n_{e}\right) t^{2}}{n U}\left(1-\frac{\sin \left(2 \pi\left(1-n_{e}\right)\right)}{2 \pi\left(1-n_{e}\right)}\right) \text { for } u \gg 1,
$$

respectively.

\section{Appendix C. Number of state representations of the spin-1/2 XXX chain and 1D Hubbard model symmetries}

The spin- $1 / 2 X X X$ chain has a global spin $S U(2)$ symmetry whose number of independent state representations is $2^{L}$. Out of such $\sum_{2 S=0 \text { (integers) }}^{L} \mathcal{N}(S)=2^{L}$ state representations, there is for a given spin $S$ a number $\mathcal{N}(S)=(2 S+1) \mathcal{N}_{\text {singlet }}(S)$ of representations. Those correspond to $(2 S+1)$ multiplet configurations and a number,

$$
\mathcal{N}_{\text {singlet }}(S)=\left(\begin{array}{c}
L \\
L / 2-S
\end{array}\right)-\left(\begin{array}{c}
L \\
L / 2-S-1
\end{array}\right),
$$

of singlet configurations. In Appendix A of Ref. [72] it is shown that for LWSs the dimension, Eq. (C.1), can alternatively be written for each $S$-fixed subspace as,

$$
\mathcal{N}_{\text {singlet }}(S)=\sum_{\left\{N_{n}\right\}} \prod_{n=1}^{\infty}\left(\begin{array}{c}
L_{n} \\
N_{n}
\end{array}\right) \text {. }
$$

As justified in Section 3.3, this expression also applies to the non-LWSs belonging to the same $S U(2)$ tower as the LWS it refers to. Here $\sum_{\left\{N_{n}\right\}}$ is a summation over all sets of the numbers $\left\{N_{n}\right\}$ corresponding to the same number of spin-singlet pairs, $\Pi=\sum_{n=1}^{\infty} n N_{n}=(L-2 S) / 2$, Eq. (64). The equality of the dimensions in Eqs. (C.1) and (C.2) confirms that the Hilbert space of the spin-1/2 XXX chain, Eq. (51), is spanned by a number $2^{L}$ of energy eigenstates. It equals that of its symmetry independent state representations.

As reported in Section 5.2, the 1D Hubbard model Hilbert-space dimension $4^{L}$ equals the number of independent state representations of its global $[S U(2) \otimes S U(2) \otimes U(1)] / Z_{2}^{2}$ symmetry. This is the second issue addressed in this Appendix. Such a model $c$ pseudoparticles, spins $1 / 2$, and $\eta$-spins $1 / 2$ configurations that generate an energy eigenstate are a superposition of local original lattice occupancy configurations. The rotated-electron occupancies of a number $L_{\eta}=L-N_{c}$ of original lattice sites separate into two degrees of freedom: Those of the $c$ lattice $U(1)$ symmetry associated with $N_{c}^{h}=L_{\eta} c$ band holes and the $\eta$-spin $S U(2)$ symmetry degrees of freedom associated with $L_{\eta}=L-N_{c} \eta$-spins $1 / 2$, respectively. The degrees of freedom of rotated-electron occupancies of the remaining $L_{S}=N_{c}$ original lattice sites also separate into two degrees of freedom: Those of the $c$ lattice $U(1)$ symmetry associated with $N_{c}=L_{S} c$ pseudoparticles and the spin $S U(2)$ symmetry degrees of freedom associated with $L_{s}=N_{c}$ spins $1 / 2$, respectively.

On the one hand, each $\alpha n$-pairs configuration occupies a number $2 n$ of original lattice sites. The set of such configurations of an energy eigenstate thus occupy a number $2 \Pi_{\alpha}=\sum_{n=1}^{\infty} 2 n N_{\alpha n}$ of original lattice sites. On the other hand, each of the $M_{\alpha}=2 S_{\alpha}$ unpaired spins $1 / 2(\alpha=s)$ and unpaired $\eta$-spins $1 / 2(\alpha=\eta)$ singly occupies an original lattice site. Similarly, each of the $N_{c} c$ pseudoparticles singly occupies an original lattice site. The remaining $N_{c}^{h}=L-N_{c}$ sites remain unoccupied in what their $c$ lattice $U(1)$ symmetry degrees of freedom is concerned. Therefore, for an energy eigenstate with fixed $N_{c} \in[0, L]$, spin $S_{s}$, and $\eta$-spin $S_{\eta}$ values, the following number of original lattice sites sum rules are fulfilled,

$$
L=\sum_{\alpha=\eta, s}\left(M_{\alpha}+2 \Pi_{\alpha}\right)=\sum_{\alpha=\eta, s}\left(2 S_{\alpha}+\sum_{n=1}^{\infty} 2 n N_{\alpha n}\right) \text { and } L=N_{c}^{h}+N_{c} .
$$

They refer to the two $S U(2)$ symmetries degrees of freedom of the $L$ original lattice sites occupancies and their $c$ lattice $U(1)$ symmetry degrees of freedom, respectively.

As for the spin-1/2 XXX chain, there is a strong requirement for each $\alpha n$-string referring to an $\alpha n$-pairs configuration. Such a configuration involves a number $2 n$ of spins $1 / 2(\alpha=s)$ and $\eta$-spins $1 / 2(\alpha=\eta)$ within $l=1, \ldots, n \alpha$-singlet pairs. The requirement under consideration is that in the dimension of any $S_{\alpha}$-fixed subspace $\mathcal{N}\left(S_{\alpha}\right)=\left(2 S_{\alpha}+1\right) \mathcal{N}_{\text {singlet }}\left(S_{\alpha}\right)$, the number of independent $\alpha$-singlet configurations $\mathcal{N}_{\text {singlet }}\left(S_{\alpha}\right)$ is exactly the same when obtained from the counting of the following two types of apparently different configurations: (i) Two $\alpha=\eta, s S U(2)$ group state representations associated with the spins $1 / 2(\alpha=s)$ and $\eta$-spins $1 / 2(\alpha=\eta)$ independent configurations with the same spin and $\eta$-spin, respectively, $S_{\alpha}$; (ii) The independent $n=1, \ldots, \infty$ bands $\left\{q_{j}\right\}$ occupancy configurations of the sets of $N_{\alpha n}$ TBA $\alpha n$-strings that obey the sum rule $\sum_{n=1}^{\infty} n N_{\alpha n}=\left(L_{\alpha}-2 S_{\alpha}\right) / 2$, Eq. (138). (The factor $\left(2 S_{\alpha}+1\right)$ in the dimension $\mathcal{N}\left(S_{\alpha}\right)=\left(2 S_{\alpha}+1\right) \mathcal{N}_{\text {singlet }}\left(S_{\alpha}\right)$ refers to the number of multiplet configurations of the $M_{\alpha}=2 S_{\alpha}$ unpaired spins $1 / 2(\alpha=s)$ and unpaired $\eta$-spins $1 / 2(\alpha=\eta)$ that are not paired within $\alpha n$-pairs configurations $\alpha$-singlet pairs.)

On the one hand, it follows directly from the $S U(2)$ symmetry algebra that number of independent spin $(\alpha=s)$ and $\eta$-spin $(\alpha=\eta) S U(2)$ symmetry state representations of the $1 \mathrm{D}$ Hubbard model in a fixed $-N_{c}$ and fixed- $S_{\alpha}$ subspace is given by $\mathcal{N}\left(S_{\alpha}, L_{\alpha}\right)=\left(2 S_{\alpha}+1\right) \mathcal{N}_{\text {singlet }}\left(S_{\alpha}, L_{\alpha}\right)$. Here,

$$
\mathcal{N}_{\text {singlet }}\left(S_{\alpha}, L_{\alpha}\right)=\left(\begin{array}{c}
L_{\alpha} \\
\Pi_{\alpha}
\end{array}\right)-\left(\begin{array}{c}
L_{\alpha} \\
\Pi_{\alpha}-1
\end{array}\right) \text { for } \alpha=\eta, s,
$$

is the corresponding number of independent spin $(\alpha=s)$ and $\eta$-spin $(\alpha=\eta) \alpha$-singlet state representations. 
On the other hand, as for the spin-1/2XXX chain, the value of the number $N_{\alpha n}^{h}=L_{\alpha n}-N_{\alpha n}$ of $\alpha n$-band holes that naturally emerges from the TBA, Eq. (130), ensures that for each $S_{\alpha}$-fixed subspace the $\alpha$-singlet dimension $\mathcal{N}_{\text {singlet }}\left(S_{\alpha}\right)$ given in Eq. (C.4) can indeed alternatively be written as,

$$
\mathcal{N}_{\text {singlet }}\left(S_{\alpha}, N_{c}\right)=\sum_{\left\{N_{\alpha n}\right\}} \prod_{n=1}^{\infty}\left(\begin{array}{c}
L_{\alpha n} \\
N_{\alpha n}
\end{array}\right) \text { for } \alpha=\eta, s .
$$

The summation $\sum_{\left\{N_{\alpha n}\right\}}$ runs here over all sets of $\alpha$-strings numbers $\left\{N_{\alpha n}\right\}$ corresponding to the same fixed spin $(\alpha=s)$ and $\eta$-spin $(\alpha=\eta) S_{\alpha}=L_{\alpha} / 2-\sum_{n=1}^{\infty} n N_{\alpha n}$. This is imposed by the exact sum rule, Eq. (138).

The demonstration in Appendix A of Ref. [72] for spin LWSs of the spin-1/2 XXX chain of the equality of the dimensions given in Eqs. (C.4) and (C.5), respectively, also applies to the 1D Hubbard model. Specifically, it applies to that model spin LWSs $(\alpha=s)$ and $\eta$-spins LWSs $(\alpha=\eta)$. This also holds for the multiplet towers of non-LWSs generated from $S_{\alpha}>0$ LWSs. All $2 S_{\alpha}+1$ states of such a tower have indeed exactly the same $\alpha$-singlet configurations as the corresponding $S_{\alpha}>0$ LWS.

In each subspace with fixed values for $L_{s}=N_{c}, L_{\eta}=N_{c}^{h}=L-N_{c}, S_{s}$, and $S_{\eta}$, there are $\mathcal{N}\left(S_{\eta}, L_{\eta}\right) \times \mathcal{N}\left(S_{s}, L_{s}\right) \times d_{c}\left(N_{c}\right)$ state representations of the $S U(2) \otimes S U(2) \otimes U(1)$ symmetry in the model two $S U(2) \otimes S U(2) \otimes U(1)] / Z_{2}^{2}$ symmetry. Here,

$$
d_{c}=\left(\begin{array}{c}
L \\
N_{c}
\end{array}\right)=\left(\begin{array}{c}
L \\
N_{c}^{h}
\end{array}\right) \text {, }
$$

gives the number of independent $c$ pseudoparticles occupancy configurations. It equals that of state representations of the $c$ lattice $U(1)$ symmetry in the subspace under consideration.

The following completeness sum rule has been obtained in Refs. [243-245] by use of the $\alpha=\eta, s$ dimensions $\mathcal{N}_{\text {singlet }}\left(S_{\alpha}, N_{c}\right)$ and $c$ dimension $d_{c}$, Eqs. (C.5) and (C.6), respectively,

$$
\begin{aligned}
4^{L}= & \sum_{\substack{N_{c}=0 \\
\text { (integers) }}}^{L} \sum_{\substack{2 S_{\eta}=0 \\
\text { (integers) }}}^{L_{\eta}=L-N_{c}} \sum_{\substack{2 S_{s}=0 \\
\text { (integers) }}}^{L_{s}=N_{c}} C\left(N_{c}, S_{\eta}, S_{S}\right) \mathcal{N}\left(S_{\eta}, L_{\eta}\right) \times \mathcal{N}\left(S_{s}, L_{s}\right) \times d_{c}\left(N_{c}\right), \\
& C\left(N_{c}, S_{\eta}, S_{s}\right)=\left|\cos \left(\frac{\pi}{2}\left(2 S_{\eta}+N_{c}\right)\right) \cos \left(\frac{\pi}{2}\left(2 S_{s}+N_{c}\right)\right)\right|=0,1 .
\end{aligned}
$$

The role of the phase factor, $C\left(N_{c}, S_{\eta}, S_{s}\right)=0,1$, is to select the allowed independent representations of the model two $S U(2)$ symmetries.

The main issue under consideration here is the equality of the dimensions given in Eqs. (C.4) and (C.5). Beyond the results of Refs. [243-245], it shows that the number of independent state representations of the 1D Hubbard model global $[S U(2) \otimes S U(2) \otimes U(1)] / Z_{2}^{2}$ symmetry exactly equals its Hilbert-space dimension $4^{L}$.

\section{Appendix D. Spin-1/2 XXX chain specific heat in the critical regime}

Here it is shown that in the $2 \mu_{B}\left|H-H_{C}\right| \ll k_{B} T$ limit where the expression, Eq. (89), is valid it is exactly the same as that obtained from the scaling function of the specific heat in the critical regime considered in Ref. [206]. The latter is denoted here by $c_{V} / L$. In the units used in that reference it reads,

$$
\frac{c_{V}}{L}=\sqrt{\frac{T}{\pi J}}\left(-\frac{3}{8} f_{3 / 2}^{s}+\frac{1}{2} \frac{\Delta}{T} f_{1 / 2}^{s}-\frac{1}{2}\left(\frac{\Delta}{T}\right)^{2} f_{-1 / 2}^{s}\right),
$$

where $f_{n}^{s}=\mathrm{Li}_{n}\left(-e^{\frac{\Delta}{T}}\right), \Delta=4 J-h$, and $\operatorname{Li}_{n}(x)=\sum_{l=1}^{\infty} x^{l} / l^{n}$.

Up to the first order in $\Delta / T \ll 1$ that the expression, Eq. (89), refers to, only the first two terms in Eq. (D.1) contribute through the following expansions,

$$
\begin{aligned}
f_{3 / 2}^{s} & \approx \mathrm{Li}_{3 / 2}(-1-\Delta / T) \approx \mathrm{Li}_{3 / 2}(-1)-\left.\frac{\Delta}{T} \frac{\partial \mathrm{Li}_{3 / 2}(x)}{\partial x}\right|_{x=-1}=-\sum_{l=1}^{\infty} \frac{(-1)^{l-1}}{l^{3 / 2}}-\frac{\Delta}{T} \sum_{l=1}^{\infty} \frac{(-1)^{l-1}}{l^{1 / 2}} \\
& =-\frac{1}{\sqrt{2}}(\sqrt{2}-1) \zeta(3 / 2)+\frac{\Delta}{T}(\sqrt{2}-1) \zeta(1 / 2), \\
f_{1 / 2}^{s} & \approx \mathrm{Li}_{1 / 2}(-1)=-\sum_{l=1}^{\infty} \frac{(-1)^{l-1}}{l^{1 / 2}}=(\sqrt{2}-1) \zeta(1 / 2) .
\end{aligned}
$$

On the one hand, the use of these expansions in the expression, Eq. (D.1), leads to the following specific heat expansion up to first order in $\Delta / T \ll 1$,

$$
\frac{c_{V}}{L}=\sqrt{\frac{T}{\pi J}}\left(\frac{3}{8 \sqrt{2}}(\sqrt{2}-1) \zeta(3 / 2)+\frac{1}{8} \frac{\Delta}{T}(\sqrt{2}-1) \zeta(1 / 2)\right) .
$$


On the other hand, the use in Eq. (89) of the coefficient expressions in Eq. (90) accounting for that $\Gamma(1 / 2)=\sqrt{\pi}$ and $\Gamma(3 / 2)=\sqrt{\pi} / 2$ leads to,

$$
\frac{c_{V}}{L}=k_{B} \sqrt{\frac{2 k_{B} T}{\pi J}}\left(\frac{3}{8 \sqrt{2}}(\sqrt{2}-1) \zeta(3 / 2)+\frac{1}{8} \frac{2 \mu_{B}\left(H_{c}-H\right)}{T}(\sqrt{2}-1) \zeta(1 / 2)\right) .
$$

The studies of Ref. [206] and those of this review use different units. From analysis of the corresponding Hamiltonian expressions, one finds that the parameters $k_{B}, J$, and $2 \mu_{B}$ used in this review correspond in the units of Ref. [206] to 1, 2J, and 1 , respectively. Hence $H_{c}=J / \mu_{B}$ becomes 4J. Finally, under the corresponding units transformations $k_{B} \rightarrow 1, J \rightarrow 2 J$, $2 \mu_{B} \rightarrow 1$, and $H_{c} \rightarrow 4 J$, the expansion, Eq. (D.4), becomes exactly that given in Eq. (D.3).

\section{Appendix E. Spin-1/2 XXX chain current expression, susceptibility, and zero-temperature stiffness}

The spin-1/2 XXX chain spin susceptibility $\chi$ and zero-temperature spin stiffness $D$ expressions in Eq. (91) are derived in this Appendix by means of procedures that resemble those of a Fermi liquid. The latter stiffness is related to the current operator expectation values. We start by confirming that in the TL their usual expression obtained from the BA for LWSs can be written in the $n$-bands holes representation, as given in Eq. (67).

Such LWSs current operator expectation values can be derived from the $\Phi / L$ dependence of the energy eigenvalues $E(\Phi / L)$ of the spin-1/2 XXX chain in a uniform vector potential $\Phi / L$, Eq. (A2) of Ref. [87]. The spin currents are then given by $\left\langle J^{z}\right\rangle=d E(\Phi / L) /\left.d(\Phi / L)\right|_{\Phi=0}$. This straightforwardly leads to $[87,88]\left\langle\hat{J}_{L W S}^{z}\left(l_{\mathrm{r}}, S\right)\right\rangle=\sum_{n=1}^{\infty} \sum_{j=1}^{L_{n}} N_{n}\left(q_{j}\right) j_{n}\left(q_{j}\right)$. Here $j_{n}\left(q_{j}\right)$ is given by $j_{n}\left(q_{j}\right)=-j_{n}^{h}\left(q_{j}\right)$, Eq. (68).

In order to confirm the equality within the TL of this $\left\langle\hat{V}_{L W S}^{z}\left(l_{\mathrm{r}}, S\right)\right\rangle$ expression and that in Eq. (67), it is useful to replace the set of $n$-bands discrete momentum values $\left\{q_{j}\right\}$ by a continuum momentum variable $q \in\left[q_{n}^{-}, q_{n}^{+}\right]$. The elementary currents $j_{n}^{h}\left(q_{j}\right)$ in Eq. (68) can then be exactly written as $j_{n}^{h}\left(q_{j}\right)=-2 J d \cos k^{n}(q) / d q$ for $q \in\left[q_{n}^{-}, q_{n}^{+}\right]$. It was used here that $d k^{n}(q) / d q=1 /\left[2 \pi \sigma^{n}\left(k^{n}(q)\right)\right]$. The equality under consideration requires that $\sum_{n=1}^{\infty} \sum_{j=1}^{L_{n}} j_{n}^{h}\left(q_{j}\right)=0$. This quantity can be written as,

$$
-\frac{L}{2 \pi} \sum_{n=1}^{\infty} \int_{q_{n}^{-}}^{q_{n}^{+}} 2 J \frac{d}{d q} \cos k^{n}(q)=-\frac{L}{2 \pi} \sum_{n=1}^{\infty} \sum_{\iota= \pm}(\iota) 2 J \cos k^{n}\left(q_{n}^{l}\right)=0 .
$$

It indeed vanishes. Here the relation $q_{n}^{-}=-q_{n}^{+}$was used.

The spin susceptibility is controlled by transitions between ground states referring to different canonical ensembles. As given in Eq. (75), those are not populated by $n$-pseudoparticles with $n>1$ pairs. Hence here we consider the spin chain in the subspace spanned by energy eigenstates that are not populated by such pseudoparticles. In that subspace the general energy functional, Eq. (77), simplifies to,

$$
\begin{aligned}
\delta E & =\sum_{j=1}^{L_{1}} \varepsilon\left(q_{j}\right) \delta N\left(q_{j}\right)+\frac{1}{L} \sum_{j=1}^{L_{1}} \sum_{j^{\prime}=1}^{L_{1}} \frac{1}{2} f\left(q_{j}, q_{j^{\prime}}\right) \delta N\left(q_{j}\right) \delta N\left(q_{j^{\prime}}\right) \\
& =\frac{L}{2 \pi} \int_{-k_{F} \uparrow}^{k_{F \uparrow}} d q \varepsilon(q) \delta N(q)+\frac{L}{4 \pi^{2}} \int_{-k_{F} \uparrow}^{k_{F \uparrow}} d q \int_{-k_{F} \uparrow}^{k_{F} \uparrow} d q^{\prime} \frac{1}{2} f\left(q, q^{\prime}\right) \delta N(q) \delta N\left(q^{\prime}\right) .
\end{aligned}
$$

Here $\delta N\left(q_{j}\right) \equiv \delta N_{1}\left(q_{j}\right), \varepsilon\left(q_{j}\right) \equiv \varepsilon_{1}\left(q_{j}\right), f\left(q_{j}, q_{j^{\prime}}\right) \equiv f_{11}\left(q_{j}, q_{j^{\prime}}\right)$. Within the TL, we have again replaced in the second expression the discrete momentum values such that $q_{j+1}-q_{j}=2 \pi / L$ by continuum momentum variables.

As in a Fermi liquid, the energy contributions of second order in the $n=1$ band momentum distribution deviations in Eq. (E.2) lead to corrections in the $n=1$ energy dispersion $\varepsilon^{0}(q) \equiv \varepsilon_{1}^{0}(q)$, Eq. (79) for $n=1$. Up to first order in these deviations one finds,

$$
\breve{\varepsilon}^{0}(q)=\varepsilon^{0}(q)+\frac{1}{2 \pi} \int_{-k_{F} \uparrow}^{k_{F \uparrow}} d q^{\prime} f\left(q, q^{\prime}\right) \delta N\left(q^{\prime}\right) .
$$

The derivation of the spin susceptibility involves small deviations $\delta q_{F}=\delta k_{F \downarrow}$ in the $n=1$ band Fermi momentum associated with transitions to ground states. For them that band momentum distribution in Eq. (75) reads,

$$
N(q)=\theta\left(k_{F \downarrow}+\delta k_{F \downarrow}-|q|\right) .
$$

Expanding this distribution around that of the initial ground state, leads to $N(q)=\theta\left(k_{F \downarrow}-|q|\right)+\delta N(q)$ where the deviation $\delta N(q)$ is given by,

$$
\delta N(q)=\delta\left(k_{F \downarrow}-|q|\right) \delta k_{F \downarrow} .
$$

Here $\delta(x)$ is the usual delta function. 
Inserting the deviations, Eq. (E.5), in the energy dispersion, Eq. (E.3), and replacing the obtained expressions in Eq. (82), which defines the magnetization curve, leads to,

$$
\frac{\partial h(m)}{\partial m}=-\frac{1}{2 \mu_{B}}\left(v+\frac{1}{2 \pi} \sum_{\iota= \pm} f\left(k_{F \downarrow}, \iota k_{F \downarrow}\right)\right) \frac{\delta k_{F \downarrow}}{\delta m}=-\frac{v\left(\xi^{0}\right)^{2}}{2 \mu_{B}} \frac{\delta k_{F \downarrow}}{\delta m}=-\frac{v^{0}}{2 \mu_{B}} \frac{\delta k_{F \downarrow}}{\delta m} .
$$

Here $v^{0}=v+\frac{1}{2 \pi} \sum_{\iota= \pm} f\left(k_{F \downarrow}, \iota k_{F \downarrow}\right)=v\left(\xi^{0}\right)^{2}$ and $\xi^{0}=1+\Phi\left(k_{F \downarrow}, k_{F \downarrow}\right)+\Phi\left(k_{F \downarrow},-k_{F \downarrow}\right)$ where $i=0,1, v \equiv v_{1}\left(k_{F \downarrow}\right)$ and $\Phi \equiv \Phi_{11}$

From the use of the relation $\delta k_{F \downarrow}=-\pi \delta m$ one finds $\delta k_{F \downarrow} / \delta m=-\pi$. The use of Eq. (E.6) with $\delta k_{F \downarrow} / \delta m=-\pi$ in the spin susceptibility expression $\chi=2 \mu_{B} /(\partial h(m) / \partial m)$ readily leads to $\chi=4 \mu_{B}^{2} /\left(\pi v_{0}\right)$. This is the expression given in Eq. (91).

The derivation of the zero-temperature spin stiffness $D$ involves again the spin-1/2 XXX chain in the subspace spanned by energy eigenstates that are not populated by pseudoparticles with $n>1$ spin-singlet pairs. For simplicity, the $n=1$ $n$-pseudoparticles are here called pseudoparticles. We consider low-frequency excitations involving a small density of pseudoparticles with momentum $q$ in the vicinity of the $n=1$ band Fermi points $\pm k_{F \downarrow}$. As in a Fermi liquid, such excitations are described by deviations $\delta N(q ; x, t)$. They depend explicitly on both position $x$ and time $t$. The corresponding momentum distribution functions reads,

$$
N(q)=\theta\left(k_{F \downarrow}-|q|\right)+\delta N(q ; x, t) .
$$

These distribution functions describe true low-frequency excitations of the spin chain. The particular form of the inhomogeneous time-dependent pseudoparticle deviations that describe these excitations can be obtained by solving kinetic equations. Those are introduced below.

Now the energy dispersion $\varepsilon\left(q_{j}\right) \equiv \varepsilon_{1}\left(q_{j}\right)$ in Eq. (79) for $n=1$ becomes a local function of the pseudoparticle deviations $\delta N(q ; x, t)$. To first order in these deviations it is given by,

$$
\breve{\varepsilon}(q ; x, t)=\varepsilon(q)+\frac{1}{2 \pi} \int_{-k_{F \uparrow}}^{k_{F} \uparrow} d q^{\prime} f\left(q, q^{\prime}\right) \delta N\left(q^{\prime} ; x, t\right) .
$$

To compute the flow of pseudoparticles through each side of a small volume element (1D segment), one considers the balance of the flow inward and outward. This leads to the following kinetic equation,

$$
\frac{\partial N(q ; x, t)}{\partial t}+\frac{\partial N(q ; x, t)}{\partial x} \frac{\partial \breve{\varepsilon}(q ; x, t)}{\partial q}-\frac{\partial N(q ; x, t)}{\partial q} \frac{\partial \breve{\varepsilon}(q ; x, t)}{\partial x}=0 .
$$

It is useful to introduce a weak inhomogeneous magnetic-field probe $\frac{1}{2} h(x, t)$. It is associated with the system conserved spin projection $S^{z}$. This requires an additional term on the left-hand side of Eq. (E.9). It is given by $\frac{\partial N(q ; x, t)}{\partial q} \mathcal{F}(q ; x, t)$. Here $\mathcal{F}(q ; x, t)=-\frac{\partial \frac{1}{2} h(x, t)}{\partial x}$ is the force felt by the pseudoparticles due to the applied magnetic-field probe, $\frac{1}{2} h(x, t)$.

The expression in Eq. (E.8) is only valid for excitations involving a small density of pseudoparticles. From the use of Eq. (E.7) in the kinetic equation, Eq. (E.9), keeping contributions up to first order in the deviations and introducing the term associated with the coupling to the external probe, we obtain,

$$
\frac{\partial \delta N(q ; x, t)}{\partial t}+v(q) \frac{\partial \delta N(q ; x, t)}{\partial x}-\frac{\partial N^{0}(q)}{\partial q}\left(\frac{1}{2 \pi} \int_{-k_{F} \uparrow}^{k_{F \uparrow}} d q^{\prime} f\left(q, q^{\prime}\right) \frac{\partial \delta N\left(q^{\prime} ; x, t\right)}{\partial x}+\frac{\partial \frac{1}{2} h(x, t)}{\partial x}\right)=0 .
$$

Here $N^{0}(q)=\theta\left(k_{F \downarrow}-|q|\right)$.

We now consider excitations that are periodic in space and time with wave vector $k$ and angular frequency $\omega$. They are characterized by the distribution function,

$$
\delta N(q ; x, t)=\delta N(q ; k, \omega) e^{i(k x-\omega t)}+\text { c.c. . }
$$

The magnetic-field probe $h(x, t) / 2$ is also assumed to be periodic in space and time, $h(x, t) / 2=[h(k, \omega) / 2] e^{i(k x-\omega t)}+$ c.c..

The real part of the spin conductivity can be written for $\omega \rightarrow 0$ as [177],

$$
\operatorname{Re} \sigma(\omega)=\operatorname{Re}\left(\lim _{k \rightarrow 0} i\left(\frac{1}{2}\right)^{2} \frac{\omega}{k^{2}} \chi(k, \omega)\right) .
$$

$\chi(k, \omega)$ is here the response function to the magnetic-field probe, $\chi(k, \omega)=\delta\left\langle S^{z}(k, \omega)\right\rangle /[h(k, \omega) / 2]$, for small $k$ and low $\omega$. To derive this response function one uses the distribution, Eq. (E.11), and the above applied magnetic-field probe $h(x, t) / 2$ in the kinetic equation, Eq. (E.10). The solution of the obtained equation leads after some manipulations to the following form for the response function $\chi(k, \omega)$ that is valid for small $k$ and low $\omega$,

$$
\chi(k, \omega)=-\frac{1}{\pi} \frac{(4 k)^{2} v^{1}}{v^{2} k^{2}-(\omega+i \delta)} .
$$

Here $v^{1}=v+\frac{1}{2 \pi} \sum_{\iota= \pm}(\iota) f\left(k_{F \downarrow}, \iota k_{F \downarrow}\right)=v\left(\xi^{1}\right)^{2}$. 
Finally, from the use of this expression on the right-hand side of Eq. (E.12), one arrives after some straightforward algebra to $\operatorname{Re} \sigma(\omega)=4 v^{1} \delta(\omega)$. From the equality $2 \pi D \delta(\omega)=4 v^{1} \delta(\omega)$ where $D$ is the spin stiffness, one finds that at zero temperature it reads $D=2 v_{1} / \pi$. This is the expression given in Eq. (91).

\section{Appendix F. The 1D Hubbard model three fractionalized effective lattices}

In this Appendix the 1D Hubbard model $c$ effective lattice and the squeezed spin and $\eta$-spin spin effective lattices considered in Section 4.3 for $u>0$ are shown to naturally emerge from the $u \rightarrow \infty$ model properties. Such properties refer to the electron occupancy configurations that generate the states $\left|l_{\mathrm{r}}, l_{\eta s}, \infty\right\rangle$. Such properties apply for $u>0$ to the rotated electrons that populate the corresponding states $\left|l_{\mathrm{r}}, l_{\eta s}, u\right\rangle=\hat{V}^{\dagger}\left|l_{\mathrm{r}}, l_{\eta s}, \infty\right\rangle$ of the same $V(u)$-set.

In the $u \rightarrow \infty$ limit the rotated electrons become electrons and the rotated-electron numbers apply as well to electron occupancies. The corresponding electron spatial coordinates are used in Eq. (2.23) of Ref. [119] for the wave functions of the LWSs $\left|l_{\mathrm{r}}, l_{\eta s}^{0}, \infty\right\rangle$. Here it is denoted by $\Psi_{l_{\mathrm{r}}, l_{\eta}, \infty}, \infty\left(x^{s}, \ldots ; x^{d}, \ldots ; x^{s \downarrow}, \ldots\right)=\left\langle x^{s}, \ldots ; x^{d}, \ldots ; x^{s \downarrow}, \ldots \mid l_{\mathrm{r}}, l_{\eta s}^{0}, \infty\right\rangle$. In their argument, $x^{s}, \ldots ; x^{d}, \ldots ; x^{s \downarrow}, \ldots$ is a shorten notation for the spatial coordinates $x^{s}, \ldots, x_{N_{R}^{s, 0}}^{s} ; x^{d}, \ldots, x_{N_{R,-1 / 2}^{n, 0}}^{d} ; x^{s \downarrow}, \ldots, x_{N_{R,-1 / 2}^{s \downarrow}}^{s, 0}$ of the $N_{R}^{s, 0}$ singly occupied sites, $N_{R,-1 / 2}^{\eta, 0}$ doubly occupied sites, and $N_{R,-1 / 2}^{s, 0}$ spin-down singly occupied sites, respectively.

One straightforwardly finds that, given two arbitrary operators $\tilde{M}=\hat{V}^{\dagger} \hat{M} \hat{V}$ and $\tilde{N}=\hat{V}^{\dagger} \hat{N}_{e} \hat{V}$, the matrix-element relations $\left\langle l_{\mathrm{r}}, l_{\eta s}, u|\tilde{M} \tilde{N}| 0_{\text {elec }}\right\rangle=\left\langle l_{\mathrm{r}}, l_{\eta s}, \infty\left|\hat{M} \hat{N}_{e}\right| 0_{\text {elec }}\right\rangle$ and $\left\langle l_{\mathrm{r}}, l_{\eta s}, u|\tilde{M} \tilde{N}| l_{\mathrm{r}}^{\prime}, l_{\eta s}^{\prime}, u\right\rangle=\left\langle l_{\mathrm{r}}, l_{\eta s}, \infty\left|\hat{M}_{\hat{N}}\right| \hat{N}_{\mathrm{r}}, l_{\eta s}^{\prime}, \infty\right\rangle$ hold. Here the electron vacuum invariance $\hat{V}\left|0_{\text {elec }}\right\rangle=\left|0_{\text {elec }}\right\rangle$ and the $V(u)$-set of states transformation $\left|l_{\mathrm{r}}, l_{\eta s}, u\right\rangle=\hat{V}^{\dagger}\left|l_{\mathrm{r}}, l_{\eta s}, \infty\right\rangle$ were used. These matrix-element relations reveal that finite- $u$ correlators of two operators, $\tilde{M}$ and $\tilde{N}$, exactly equal those of the corresponding two unrotated operators, $\hat{M}$ and $\hat{N}_{e}$, in the $u \rightarrow \infty$ limit.

We denote by $\Psi_{l_{\mathrm{r}}, l_{\eta}, r t}\left(x^{s}, \ldots ; x^{d}, \ldots ; x^{s \downarrow}, \ldots\right)=\left\langle x^{s}, \ldots ; x^{d}, \ldots ; x^{s \downarrow}, \ldots \mid l_{\mathrm{r}}, l_{\eta s}^{0}, u\right\rangle$ the $N_{e}$-rotated-electron wave function of a finite- $u$ LWS $\left|l_{\mathrm{r}}, l_{\eta s}^{0}, u\right\rangle=\hat{V}^{\dagger}\left|l_{\mathrm{r}}, l_{\eta s}^{0}, \infty\right\rangle$. It is a function of corresponding rotated-electron spatial coordinates. From the use of the above matrix-element relations one finds that $\Psi_{l_{\mathrm{r}}, l_{\eta s}, r t}\left(x^{s}, \ldots ; x^{d}, \ldots ; x^{s \downarrow}, \ldots\right)=\Psi_{l_{\mathrm{r}}, l_{\eta_{s}}^{0}, \infty}\left(x^{s}, \ldots ; x^{d}, \ldots ; x^{s \downarrow}, \ldots\right)$ for the whole finite- $u$ range.

Combining the equalities $\Psi_{l_{\mathrm{r}}, l_{s}, r t}=\Psi_{l_{\mathrm{r}}, l_{\eta_{s}}^{0}, r t}$ for finite $u$ and $\Psi_{l_{\mathrm{r}}, l_{\eta_{s}}^{0}, r t}=\Psi_{l_{\mathrm{r}}, l_{\eta_{s}}^{0}, \infty}$ with the expression given in Eq. (2.23) of Ref. [119] for $\Psi_{l_{\mathrm{r}}, l_{n,}^{0}, \infty}$ leads to,

$$
\Psi_{l_{\mathrm{r}}, l_{\eta s}, r t}\left(x^{s}, \ldots ; x^{d}, \ldots ; x^{s \downarrow}\right)=\frac{1}{\sqrt{\mathcal{C}_{r t}}}\left(\phi_{U(1)}^{c}\left(x^{s}, \ldots\right) \times \phi_{S U(2)}^{\eta}\left(x^{d}, \ldots\right) \times \phi_{S U(2)}^{s}\left(x^{s \downarrow}, \ldots\right)\right) .
$$

Here $\phi_{U(1)}^{c}\left(x^{s}, \ldots\right)=(-1)^{Q} \operatorname{det}\left(e^{i k_{p j}^{\infty} x_{0 j}^{s}}\right)$ is a $c$ pseudofermion Slater determinant. The $c$ pseudofermions occupy exactly the same sites as the $c$ pseudoparticles. Moreover, $\phi_{S U(2)}^{\eta}\left(x^{d}, \ldots\right)=(-1)^{\left(N^{0}-2 S_{c}\right) / 2} \phi_{1}\left(x^{d}, \ldots\right)$ refers to the $\eta$-spins $1 / 2$, $\phi_{S U(2)}^{s}\left(x^{s \downarrow}, \ldots\right)=\phi_{2}\left(x^{s \downarrow}, \ldots\right)$ corresponds to the spins $1 / 2$, and $\mathcal{C}_{r t}$ is a normalization constant. Since such wave functions refer to rotated electrons, the BA rapidities on which they depend are $u$ independent. They are actually those of the electrons in the $u \rightarrow \infty$ limit.

For a LWS the spatial coordinates of the wave functions $\phi_{U(1)}^{c}\left(x^{s}, \ldots\right), \phi_{S U(2)}^{\eta}\left(x^{d}, \ldots\right)$, and $\phi_{S U(2)}^{s}\left(x^{s \downarrow}, \ldots\right)$ refer in the TL to well-defined lattice sites subsets of the $c$ effective lattice, squeezed $\eta$-spin effective lattice, and squeezed spin effective, respectively. The $c$ effective lattice is identical to the original lattice. On the contrary, the numbers of sites $L_{\eta}$ and $L_{s}$ of the squeezed $\eta$-spin and spin effective lattices, respectively, are in general smaller than $L$, as given in Eq. (123).

The subset of the $c$ effective lattice $N_{c}$ sites of spatial coordinates $x^{s}, \ldots$ in the argument of $\phi_{U(1)}^{c}\left(x^{s}, \ldots\right)$ refers to those occupied. The remaining $N_{c}^{h}=L-N_{c}$ sites whose spatial coordinates are uniquely defined are unoccupied. The $L_{\eta}=N_{c}^{h}$ sites of the squeezed $\eta$-spin effective lattice correspond to those of the original lattice that have the same spatial coordinates as the $c$ effective lattice unoccupied sites. The subset of $N_{R,-1 / 2}^{\eta, 0}$ sites of spatial coordinates $x^{d}, \ldots$ in $\phi_{S U(2)}^{\eta}\left(x^{d}, \ldots\right)$ corresponds to those occupied by $\eta$-spins of projection $-1 / 2$. The remaining $N_{R,+1 / 2}^{\eta, 0}=L_{\eta}-N_{R,-1 / 2}^{\eta, 0}$ sites whose spatial coordinates are those left over are occupied by $\eta$-spins of projection $+1 / 2$.

Similarly, the $L_{s}=N_{c}$ sites of the squeezed spin effective lattice refer to those of the original lattice that have the same spatial coordinates as the $c$ effective lattice occupied sites. The subset of $N_{R,-1 / 2}^{s, 0}$ sites of spatial coordinates $x^{s \downarrow}, \ldots$ in $\phi_{S U(2)}^{s}\left(x^{s \downarrow}, \ldots\right)$ corresponds to those occupied by spins of projection $-1 / 2$. The remaining $N_{R,+1 / 2}^{s, 0}=L_{s}-N_{s,-1 / 2}^{\eta, 0}$ sites whose spatial coordinates are uniquely defined are occupied by spins of projection $+1 / 2$. Within the present TL, the spin effective lattice (and $\eta$-spin effective lattice) has $j=1, \ldots, L_{s}$ sites located at $x=a_{s} j$. (and $j=1, \ldots, L_{\eta}$ sites located at $x=a_{\eta} j$ where $j=1, \ldots, L_{\eta}$.) The spacings $a_{s}$ and $a_{\eta}$ are given in Eq. (124).

\section{Appendix G. The 1D Hubbard model $s 1$ pseudoparticle operator representation}

The $s 1$ pseudoparticle operator representation considered in Section 5.2 for the 1D Hubbard model is valid in the extended Takahashi subspaces. A related Takahashi subspace is spanned by all LWSs with the same fixed values for the set of numbers $N_{c}$ and $\left\{N_{\alpha n}\right\}$ for $\alpha=\eta, s$ and $n=1, \ldots, \infty$. The corresponding extended Takahashi subspace is spanned by such LWSs and the 
$\left(2 S_{\eta}+1\right) \times\left(2 S_{S}+1\right)-1$ non-LWSs generated from each of them as given in Eq. (110). A general $\alpha n$ pseudoparticle operator representation can be introduced for the $1 \mathrm{D}$ Hubbard model in an extended Takahashi subspace. For the results reviewed in this paper only the $s 1$ pseudoparticle operator representation is though needed.

That in fixed- $L_{s 1}$ extended Takahashi subspaces the local $s 1$ pseudoparticle operators obey a fermionic algebra, can be confirmed in terms of their statistical interactions [262]. The local $s 1$ pseudoparticle creation and annihilation operators may be written as,

$$
f_{j, s 1}^{\dagger}=e^{i \phi_{j, s 1}} g_{j, s 1}^{\dagger} \text { and } f_{j, s 1}=\left(f_{j, s 1}^{\dagger}\right)^{\dagger} \text { for } j=1, \ldots, L_{s 1} \text {. }
$$

Here $\phi_{j, s 1}=\sum_{j^{\prime} \neq j_{j}, s 1}^{\dagger}$ and the operator $g_{j, s 1}^{\dagger}$ obeys a hard-core bosonic algebra. This algebra is justified by the corresponding statistical interaction vanishing for the model in fixed- $L_{s 1}$ extended Takahashi subspaces. The $s 1$ effective lattice has been constructed inherently to that algebra being of hard-core type for the operators $g_{j, s 1}^{\dagger}$ and $g_{j, s 1}$. Therefore, through a JordanWigner transformation, $f_{j, s 1}^{\dagger}=e^{i \phi_{j, s 1}} g_{j, s 1}^{\dagger}$ (see for instance Ref. [282]), the operators $f_{j, s 1}^{\dagger}$ and $f_{j, s 1}=\left(f_{j, s 1}^{\dagger}\right)^{\dagger}$ in Eq. $(\mathrm{G} .1)$ obey indeed a Fermionic algebra, Eq. (141).

\section{Appendix H. Integral equations that define the 1D Hubbard model pseudofermion rapidity phase shifts}

The rapidity phase shifts, Eq. (154), are in units of $2 \pi$ uniquely defined by the following integral equations [99],

$$
\begin{aligned}
\bar{\Phi}_{s 1 c}\left(r, r^{\prime}\right) & =-\frac{1}{\pi} \arctan \left(r-r^{\prime}\right)+\int_{-r_{s}^{0}}^{r_{s}^{0}} d r^{\prime \prime} G\left(r, r^{\prime \prime}\right) \bar{\Phi}_{s 1 c}\left(r^{\prime \prime}, r^{\prime}\right), \\
\bar{\Phi}_{s 1 \eta n}\left(r, r^{\prime}\right) & =-\frac{1}{\pi^{2}} \int_{-r_{c}^{0}}^{r_{c}^{0}} d r^{\prime \prime} \frac{\arctan \left(\frac{r^{\prime \prime}-r^{\prime}}{n}\right)}{1+\left(r-r^{\prime \prime}\right)^{2}}+\int_{-r_{s}^{0}}^{r_{s}^{0}} d r^{\prime \prime} G\left(r, r^{\prime \prime}\right) \bar{\Phi}_{s 1 \eta n}\left(r^{\prime \prime}, r^{\prime}\right), \\
\bar{\Phi}_{s 1 s n}\left(r, r^{\prime}\right) & =\frac{\delta_{1, n}}{\pi} \arctan \left(\frac{r-r^{\prime}}{2}\right)+\frac{\left(1-\delta_{1, n}\right)}{\pi}\left(\arctan \left(\frac{r-r^{\prime}}{n-1}\right)+\arctan \left(\frac{r-r^{\prime}}{n+1}\right)\right) \\
& -\frac{1}{\pi^{2}} \int_{-r_{c}^{0}}^{r_{c}^{0}} d r^{\prime \prime} \frac{\arctan \left(\frac{r^{\prime \prime}-r^{\prime}}{n}\right)}{1+\left(r-r^{\prime \prime}\right)^{2}}+\int_{-r_{s}^{0}}^{r_{s}^{0}} d r^{\prime \prime} G\left(r, r^{\prime \prime}\right) \bar{\Phi}_{s 1 s 1}\left(r^{\prime \prime}, r^{\prime}\right),
\end{aligned}
$$

$$
\bar{\Phi}_{c c}\left(r, r^{\prime}\right)=\frac{1}{\pi} \int_{-r_{s}^{0}}^{r_{s}^{0}} d r^{\prime \prime} \frac{\bar{\Phi}_{s 1 c}\left(r^{\prime \prime}, r^{\prime}\right)}{1+\left(r-r^{\prime \prime}\right)^{2}}
$$$$
\bar{\Phi}_{c \alpha n}\left(r, r^{\prime}\right)=-\frac{1}{\pi} \arctan \left(\frac{r-r^{\prime}}{n}\right)+\frac{1}{\pi} \int_{-r_{s}^{0}}^{r_{s}^{0}} d r^{\prime \prime} \frac{\bar{\Phi}_{s 1 \alpha n}\left(r^{\prime \prime}, r^{\prime}\right)}{1+\left(r-r^{\prime \prime}\right)^{2}}, \quad \text { where } \alpha=\eta, s \text {, }
$$$$
\bar{\Phi}_{\eta n c}\left(r, r^{\prime}\right)=\frac{1}{\pi} \arctan \left(\frac{r-r^{\prime}}{n}\right)-\frac{1}{\pi} \int_{-r_{c}^{0}}^{+r_{c}^{0}} d r^{\prime \prime} \frac{\bar{\Phi}_{c c}\left(r^{\prime \prime}, r^{\prime}\right)}{n\left[1+\left(\frac{r-r^{\prime \prime}}{n}\right)^{2}\right]},
$$$$
\bar{\Phi}_{\eta n \eta n^{\prime}}\left(r, r^{\prime}\right)=\frac{1}{2 \pi} \Theta_{n, n^{\prime}}\left(r-r^{\prime}\right)-\frac{1}{\pi} \int_{-r_{c}^{0}}^{+r_{c}^{0}} d r^{\prime \prime} \frac{\bar{\Phi}_{c \eta n^{\prime}}\left(r^{\prime \prime}, r^{\prime}\right)}{n\left[1+\left(\frac{r-r^{\prime \prime}}{n}\right)^{2}\right]},
$$$$
\bar{\Phi}_{\eta n s n^{\prime}}\left(r, r^{\prime}\right)=-\frac{1}{\pi} \int_{-r_{c}^{0}}^{+r_{c}^{0}} d r^{\prime \prime} \frac{\bar{\Phi}_{c s n^{\prime}}\left(r^{\prime \prime}, r^{\prime}\right)}{n\left[1+\left(\frac{r-r^{\prime \prime}}{n}\right)^{2}\right]},
$$

$$
\bar{\Phi}_{s n c}\left(r, r^{\prime}\right)=-\frac{1}{\pi} \arctan \left(\frac{r-r^{\prime}}{n}\right)+\frac{1}{\pi} \int_{-r_{c}^{0}}^{r_{c}^{0}} d r^{\prime \prime} \frac{\bar{\Phi}_{c, c}\left(r^{\prime \prime}, r^{\prime}\right)}{n\left[1+\left(\frac{r-r^{\prime \prime}}{n}\right)^{2}\right]}-\int_{-r_{s}^{0}}^{r_{s}^{0}} d r^{\prime \prime} \bar{\Phi}_{s 1 c}\left(r^{\prime \prime}, r^{\prime}\right) \frac{\Theta_{n, 1}^{[1]}\left(r-r^{\prime \prime}\right)}{2 \pi} \text { for } n>1 \text {, }
$$

$$
\bar{\Phi}_{s n \eta n^{\prime}}\left(r, r^{\prime}\right)=\frac{1}{\pi} \int_{-r_{c}^{0}}^{r_{c}^{0}} d r^{\prime \prime} \frac{\bar{\Phi}_{c, \eta n^{\prime}}\left(r^{\prime \prime}, r^{\prime}\right)}{n\left[1+\left(\frac{r-r^{\prime \prime}}{n}\right)^{2}\right]}-\int_{-r_{s}^{0}}^{r_{s}^{0}} d r^{\prime \prime} \bar{\Phi}_{s 1 \eta n^{\prime}}\left(r^{\prime \prime}, r^{\prime}\right) \frac{\Theta_{n, 1}^{[1]}\left(r-r^{\prime \prime}\right)}{2 \pi} \text { for } n>1
$$

$$
\bar{\Phi}_{s n s n^{\prime}}\left(r, r^{\prime}\right)=\frac{1}{2 \pi} \Theta_{n, n^{\prime}}\left(r-r^{\prime}\right)+\frac{1}{\pi} \int_{-r_{c}^{0}}^{r_{c}^{0}} d r^{\prime \prime} \frac{\bar{\Phi}_{c s n^{\prime}}\left(r^{\prime \prime}, r^{\prime}\right)}{n\left[1+\left(\frac{r-r^{\prime \prime}}{n}\right)^{2}\right]}-\int_{-r_{s}^{0}}^{r_{s}^{0}} d r^{\prime \prime} \bar{\Phi}_{s 1 s n^{\prime}}\left(r^{\prime \prime}, r^{\prime}\right) \frac{\Theta_{n, 1}^{[1]}\left(r-r^{\prime \prime}\right)}{2 \pi} .
$$


In the above equations, the parameters $r_{c}^{0}$ and $r_{s}^{0}$ are given in Eq. (153), the functions $\Theta_{n, n^{\prime}}(x)$ and $\Theta_{n, n^{\prime}}^{[1]}(x)$ are defined in Eqs. (B.1) and (B.2) of Appendix B, respectively, and the kernel $G\left(r, r^{\prime}\right)$ reads [92],

$$
\begin{aligned}
G\left(r, r^{\prime}\right) & =-\frac{1}{2 \pi}\left[\frac{1}{1+\left(\left(r-r^{\prime}\right) / 2\right)^{2}}\right] \\
& \times\left(1-\frac{1}{2 \pi} \sum_{\iota= \pm 1}(\iota)\left(\arctan \left(r+\iota r_{c}^{0}\right)+\arctan \left(r^{\prime}+\iota r_{c}^{0}\right)+\frac{1}{\left(r-r^{\prime}\right)} \ln \frac{1+\left(r+\iota r_{c}^{0}\right)^{2}}{1+\left(r^{\prime}+\iota r_{c}^{0}\right)^{2}}\right)\right) .
\end{aligned}
$$

The relation of the $m \rightarrow 0$ parameter $\xi_{0}$ in Eq. (163) to phase shifts is obtained by combining such equation with Eq. (162). This gives,

$$
\begin{aligned}
& \lim _{m \rightarrow 0} Z^{1}=\left[\begin{array}{cc}
\xi_{0} & \xi_{0} / 2 \\
0 & 1 / \sqrt{2}
\end{array}\right]=\left[\begin{array}{ll}
1 & 0 \\
0 & 1
\end{array}\right]+\sum_{\iota= \pm}\left[\begin{array}{ll}
\Phi_{c c}\left(\iota 2 k_{F}, 2 k_{F}\right) & \Phi_{c s 1}\left(\iota 2 k_{F}, k_{F}\right) \\
\Phi_{s 1 c}\left(\iota k_{F}, 2 k_{F}\right) & \Phi_{s 1 s 1}\left(\iota k_{F}, k_{F}\right)
\end{array}\right], \\
& \lim _{m \rightarrow 0} Z^{0}=\left[\begin{array}{cc}
1 / \xi_{0} & 0 \\
-\xi_{0} / 2 & \sqrt{2}
\end{array}\right]=\left[\begin{array}{ll}
1 & 0 \\
0 & 1
\end{array}\right]+\sum_{\iota= \pm}(\iota)\left[\begin{array}{cc}
\Phi_{c, c}\left(\iota 2 k_{F} 2 k_{F}\right) & \Phi_{c s 1}\left(\iota 2 k_{F}, k_{F}\right) \\
\Phi_{s 1, c}\left(\iota k_{F} 2 k_{F}\right) & \Phi_{s 1 s 1}\left(\iota k_{F}, k_{F}\right)
\end{array}\right] .
\end{aligned}
$$

Here $Z^{0}=\left(\left(Z^{1}\right)^{-1}\right)^{T}$. In the above equations we have accounted for that the $c$ and $s 1$ band Fermi points in Eq. (147) read $q_{F C}=2 k_{F}$ and $q_{F s 1}=k_{F}$, respectively, in the $m \rightarrow 0$ limit

Conversely, in the $m \rightarrow 0$ limit the $c$ and $s 1$ pseudofermion phase shifts with both momenta at the Fermi points can be expressed in terms of only the parameter $\xi_{0}$ as follows,

$$
\begin{aligned}
2 \pi \Phi_{c c}\left(\iota 2 k_{F}, 2 k_{F}\right)=\iota 2 \pi \Phi_{c, c}\left(2 k_{F}, \iota 2 k_{F}\right) & =\frac{\pi\left(\xi_{0}-1\right)^{2}}{\xi_{0}} \text { for } \iota=+, \\
& =\frac{\pi\left(\xi_{0}^{2}-1\right)}{\xi_{0}} \text { for } \iota=-, \\
2 \pi \Phi_{c s 1}\left(\iota 2 k_{F}, k_{F},\right)=\iota 2 \pi \Phi_{c s 1}\left(2 k_{F}, \iota k_{F},\right) & =\frac{\pi}{2} \xi_{0} \text { for } \iota= \pm .
\end{aligned}
$$

\section{Appendix I. $\delta S_{\alpha}=\mp n \alpha$-multiplet elementary processes associated with creation and annihilation of one $\alpha n$ pseudoparticle in the spin density $m \rightarrow n_{e}$ limit}

For electronic densities in the interval $\left.n_{e} \in\right] 0,1\left[\right.$, spin density $m \rightarrow n_{e}$, and the whole $u>0$ range the energy dispersions $\varepsilon_{\alpha n}^{0}\left(q_{j}\right)$ and $\varepsilon_{\alpha n}\left(q_{j}\right)$ are for small momentum $q_{j}(\alpha=s)$ and small momentum deviations $\left(q_{j} \mp q_{\eta n}\right)(\alpha=\eta)$ given by,

$$
\begin{aligned}
& \varepsilon_{s n}^{0}\left(q_{j}\right) \approx \frac{q_{j}^{2}}{2 m_{s n}^{*}}-W_{s n}=\frac{q_{j}^{2}}{2 m_{s n}^{*}}-W_{s n}^{\text {pair }} \text { for } q_{j} \approx 0 \text { and } \varepsilon_{s n}\left(q_{j}\right)=\varepsilon_{s n}^{0}\left(q_{j}\right)+n 2 \mu_{B} H, \\
& \varepsilon_{\eta n}^{0}\left(q_{j}\right) \approx \frac{\left(q_{j} \mp q_{\eta n}\right)^{2}}{2 m_{\eta n}^{*}} \text { for }\left(q_{j} \mp q_{\eta n}\right) \approx 0 \text { and } \varepsilon_{\eta n}\left(q_{j}\right)=\varepsilon_{\eta n}^{0}\left(q_{j}\right)+n 2 \mu .
\end{aligned}
$$

Here $W_{s n}=W_{s n}^{\text {pair }}$ is given in Eq. (B.10) of Appendix B.

The effective masses $m_{s n}^{*}$ and $m_{\eta n}^{*}$ in these expressions have the following analytical expressions that are functions of $n_{e}$ and $U / t$,

$$
\left.m_{s n}^{*}=\frac{n U}{4 t^{2}} \frac{\frac{\sqrt{(4 t)^{2}+n U^{2}}}{n U} \frac{1}{\pi} \arctan \left(\frac{\sqrt{(4 t)^{2}+(n U)^{2}}}{n U} \tan \left(\pi n_{e}\right)\right)}{1-\frac{\sqrt{(4 t)^{2}+(n U)^{2}}}{n U}} \frac{1}{1+\left(\frac{4 t \sin \left(\pi n_{e}\right)}{n U}\right)^{2}} \frac{\sin \left(2 \pi n_{e}\right)}{2 \arctan \left(\frac{\sqrt{(4 t)^{2}+(n U)^{2}}}{n U} \tan \left(\pi n_{e}\right)\right)} \text { for } n_{e} \in\right] 0,1\left[\text { and } m \rightarrow n_{e},\right.
$$

and

$$
\left.m_{\eta n}^{*}=\frac{n U}{4 t^{2}} \frac{\frac{\sqrt{(4 t)^{2}+n U^{2}}}{n U} \frac{1}{\pi} \arctan \left(\frac{\sqrt{(4 t)^{2}+(n U)^{2}}}{n U} \tan \left(\pi\left(1-n_{e}\right)\right)\right)}{1-\frac{\sqrt{(4 t)^{2}+(n U)^{2}}}{n U} \frac{1}{1+\left(\frac{4 t \sin \left(\pi\left(1-n_{e}\right)\right)}{n U}\right)^{2}} \frac{\sin \left(2 \pi\left(1-n_{e}\right)\right)}{2 \arctan \left(\frac{\sqrt{(4 t)^{2}+(n U)^{2}}}{n U} \tan \left(\pi\left(1-n_{e}\right)\right)\right)}} \text { for } n_{e} \in\right] 0,1\left[\text { and } m \rightarrow n_{e},\right.
$$

respectively. In the $u \rightarrow 0$ and $u \gg 1$ limits these expressions read,

$$
m_{s n}^{*}=\frac{1}{2 t} \text { for } u \rightarrow 0
$$




$$
m_{s n}^{*}=\frac{n U}{4 t^{2}} \frac{n_{e}}{\left(1-\frac{\sin \left(2 \pi n_{e}\right)}{2 \pi n_{e}}\right)} \text { for } u \gg 1,
$$

and

$$
\begin{aligned}
& m_{\eta n}^{*}=\frac{1}{2 t} \text { for } u \rightarrow 0 \\
& m_{\eta n}^{*}=\frac{n U}{4 t^{2}} \frac{\left(1-n_{e}\right)}{\left(1-\frac{\sin \left(2 \pi\left(1-n_{e}\right)\right)}{2 \pi\left(1-n_{e}\right)}\right)} \text { for } u \gg 1,
\end{aligned}
$$

respectively. In the case of the $s 1$ band, the energy dispersion, Eq. (I.1) for $\alpha n=s 1$, is that given in Eq. (168). The corresponding effective triplet mass is provided in Eq. (170).

For $h \approx H_{c}$ and electronic densities $\left.n_{e} \in\right] 0,1[$ not too close to 0 and 1 , the elementary process associated with creation of one $s n$ pseudoparticle or one $\eta n$ pseudoparticle onto the ground state leads to a spin or $\eta$-spin deviation $\delta S_{s}=-n=-1, \ldots,-L_{s} / 2$ or $\delta S_{\eta}=-n=-1, \ldots,-L_{\eta} / 2$, respectively. Under such an elementary process, a number $2 n=2,4, \ldots, L_{s}$ or $2 n=2,4, \ldots, L_{\eta}$ of initial-state unpaired spins $1 / 2$ or unpaired $\eta$-spins $1 / 2$, respectively, become paired within the final-state pseudoparticle spin-singlet $s n$-pairs configuration or $\eta$-spin-singlet $\eta n$-pairs configuration, respectively.

The opposite elementary process associated with the annihilation of one $s n$ pseudoparticle $(\alpha=s)$ or one $\eta n$ pseudoparticle $(\alpha=\eta)$ to return to the initial state, involves the breaking of all its $n \alpha$-singlet pairs. This gives rise to the emergence of $2 n$ unpaired spins $1 / 2$ or unpaired $\eta$-spins $1 / 2$, respectively, in the new final state. Such an opposite elementary process leads to a spin or $\eta$-spin deviation $\delta S_{s}=n=1, \ldots, L_{s} / 2$ or $\delta S_{\eta}=n=1, \ldots, L_{\eta} / 2$, respectively.

Each above mentioned $\delta S_{\alpha}=-n$ elementary process has a minimum excitation energy given by $\Delta_{s n}^{\min }=\varepsilon_{s n}(0)=$

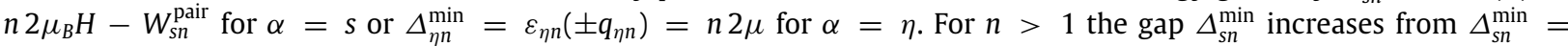
$(n-1) 2 \mu_{B} H+2 \mu_{B}\left(H-H_{c}\right) \approx(n-1) 2 \mu_{B} H$ for $u \rightarrow 0$ to $\Delta_{s n}^{\min }=(n-1 / n) 2 \mu_{B} H+2 \mu_{B}\left(H-H_{c}\right) / n \approx(n-1 / n) 2 \mu_{B} H$ for $u \gg 1$ and $\Delta_{\eta n}^{\min }$ reads $\Delta_{\eta n}^{\min }=n\left(U+4 t \cos \left(\pi n_{e}\right)\right)+n 2 \mu_{B}\left(H-H_{c}\right) \approx n\left(U+4 t \cos \left(\pi n_{e}\right)\right)$.

Hence within the low-temperature crossover critical regime considered in Section 6.2, for which $2 \mu_{B}\left|H-H_{c}\right| \ll k_{B} T$, only the spin-triplet channel associated with elementary $\delta S_{S}= \pm 1$ spin-triplet processes with $\delta S_{S}=-1$ is available. Its excitation energy reads $\Delta_{s 1}^{\min }=2 \mu_{B}\left(H-H_{c}\right)$. The next two minimum gaps read $\Delta_{s 2}^{\min }=\varepsilon_{s 2}(0)=4 \mu_{B} h-W_{s 2}^{\text {pair }}$ for a spin $\delta S_{s}=-2$ elementary process, which is the minimum gap $\Delta_{s}^{\min }$, Eqs. (166) and (167), and $\Delta_{\eta 1}^{\min }=\left(U+4 t \cos \left(\pi n_{e}\right)\right)$ for an $\eta$-spin-triplet $\delta S_{\eta}=-1$ elementary process. Those refer though to high-energy processes such that $\Delta_{s 2}^{\min } \gg 2 \mu_{B}\left(H-H_{c}\right)$ and $\Delta_{\eta 1}^{\min } \gg 2 \mu_{B}\left(H-H_{c}\right)$.

Finally, one finds that $W_{\alpha n}^{\text {pair }}$ and $m_{\alpha n}^{*}$ are related yet different quantities whose interplay partially controls the $\delta S_{\alpha}= \pm n$ elementary processes under consideration. This is achieved from inspection for electronic densities $n_{e} \in$ ]0, 1[ and spin

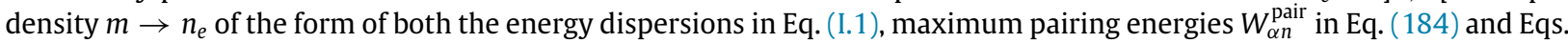
(B.10)-(B.13) of Appendix B, and effective masses $m_{\alpha n}^{*}$ in Eqs. (170) and (171) and Eqs. (I.2)-(I.5).

This applies to the spin-triplet $S_{S}= \pm 1$ elementary processes. Those contribute to the low-temperature specific heat expressions, Eqs. (172) and (173), in the crossover critical regime. It refers to a small field window $2 \mu_{B}\left|H-H_{c}\right| \ll k_{B} T$ around $H_{c}$. In this case, the spin-triplet effective mass $m_{s 1}^{*}$, Eqs. (170) and (171), and the maximum pairing energy $W_{s 1}^{\text {pair }}=$ $W_{s 1}=2 \mu_{B} H_{c}$, Eq. (169), associated with the spin-singlet configuration binding of the two paired spins $1 / 2$ within the $s 1$ pseudoparticle are different yet related quantities. They are given by $m_{s 1}^{*}=\left.\frac{\partial^{2} \varepsilon_{s 1}^{0}(q)}{\partial q^{2}}\right|_{q=0}$ and $W_{s 1}=\left(\varepsilon_{s 1}^{0}\left(q_{s 1}\right)-\varepsilon_{s 1}^{0}(0)\right)=$ $-\varepsilon_{s 1}^{0}(0)=2 \mu_{B} H_{c}$, respectively. Here $q_{s 1}=2 k_{F}=\pi n_{e}$ for $m \rightarrow n_{e}$.

\section{Appendix J. Derivation of the $\beta=c, s 1$ pseudofermion spectral function within the TL and example of momentum- dependent exponents}

The 1D Hubbard model $\beta=c, s 1$ pseudofermion spectral function general expression, Eq. (193), involves the $\beta=c, s 1$ lowest peak weight $A_{\beta}^{(0,0)}$ and relative weight $a_{\beta}=a_{\beta}\left(m_{\beta,+1}, m_{\beta,-1}\right)$. After a suitable algebra similar to that reported in Ref. [158] for the $u \rightarrow \infty$ spin-less fermion spectral function, one finds that the former weight refers to a Slater determinant of $\beta=c, s 1$ pseudofermion operators. It involves $\beta=c, s 1$ pseudofermion anticommutators associated with two $\beta$ pseudofermions of canonical momentum $\bar{q}_{j}$ and $\bar{q}_{j^{\prime}}$, respectively. Here $\bar{q}_{j}$ and $\bar{q}_{j^{\prime}}=q_{j^{\prime}}$ correspond to a PS excited state and the corresponding ground-state $\beta$ band, respectively. One then finds the anticommutators [51],

$$
\left\{f_{\bar{q}_{j}, \beta}^{\dagger}, f_{\bar{q}_{j^{\prime}}, \beta}\right\}=\frac{1}{L_{\beta}} e^{-i\left(\bar{q}_{j}-\bar{q}_{j^{\prime}}\right) / 2} e^{i 2 \pi \Phi_{\beta}^{T}\left(q_{j}\right) / 2} \frac{\sin \left(2 \pi \Phi_{\beta}^{T}\left(q_{j}\right) / 2\right)}{\sin \left(\left[\bar{q}_{j}-\bar{q}_{j^{\prime}}\right] / 2\right)} \text { and } 2 \pi \Phi_{\beta}^{T}\left(q_{j}\right)=2 \pi \Phi_{\beta}^{0}+2 \pi \Phi_{\beta}\left(q_{j}\right),
$$

and $\left\{f_{\bar{q}_{j}, \beta}^{\dagger}, f_{\bar{q}_{j^{\prime}}, \beta}^{\dagger}\right\}=\left\{f_{\bar{q}_{j}, \beta}, f_{\bar{q}_{j^{\prime}}, \beta}\right\}=0$. Here $2 \pi \Phi_{\beta}^{0}$, Eq. (150), is the non-scattering part of the overall $\beta$ pseudofermion phase shift denoted by $2 \pi \Phi_{\beta}^{T}\left(q_{j}\right)$ in this equation. 
The use of the Slater determinant of $\beta=c, s 1$ pseudofermion operators that involves such anticommutators leads after some algebra to the following general expression for the $\beta=c, s 1$ lowest peak weight $A_{\beta}^{(0,0)}[51,63,64]$,

$$
\begin{aligned}
A_{\beta}^{(0,0)} & =\left(\frac{1}{L}\right)^{2 N_{\beta}^{\odot}} \prod_{j=1}^{L_{\beta}} \sin ^{2}\left(\frac{\pi}{2}\left(1-\left(1-2 \Phi_{\beta}^{T}\left(q_{j}\right)\right) N_{\beta}^{\odot}\left(q_{j}\right)\right)\right) \prod_{j=1}^{L_{\beta}-1}\left(\sin \left(\frac{\pi j}{L}\right)\right)^{2\left(L_{\beta}-j\right)} \\
& \times \prod_{i=1}^{L_{\beta}} \prod_{j=1}^{L_{\beta}} \theta(j-i) \sin ^{2}\left(\frac{\pi}{2}\left(1-\left(1-\frac{\left(2(j-i)+2 \Phi_{\beta}^{T}\left(q_{j}\right)-2 \Phi_{\beta}^{T}\left(q_{i}\right)\right)}{L}\right) N_{\beta}^{\odot}\left(q_{j}\right) N_{\beta}^{\odot}\left(q_{i}\right)\right)\right) \\
& \times \prod_{i=1}^{L_{\beta}} \prod_{j=1}^{L_{\beta}} \frac{1}{\sin ^{2}\left(\frac{\pi}{2}\left(1-\left(1-\frac{2(j-i)+2 \Phi_{\beta}^{T}\left(q_{j}\right)}{L}\right) N_{\beta}^{\odot}\left(q_{i}\right) N_{\beta}^{\odot}\left(q_{j}\right)\right)\right)} \text { where } \beta=c, s 1 .
\end{aligned}
$$

The numbers of $\beta=c, s 1$ band discrete momentum values, $L_{\beta}, \beta=c, s 1$ pseudofermions, $N_{\beta}^{\odot}=\sum_{j=1}^{L_{\beta}} N_{\beta}^{\odot}\left(q_{j}\right)$, and the corresponding $\beta$ band momentum distribution function, $N_{\beta}^{\odot}\left(q_{j}\right)$, are in this expression those of the PS excited state generated by the processes (A) and (B) as defined in Section 7.2. Moreover, $\Phi_{\beta}^{T}\left(q_{j}\right)$ is the phase-shift functional in Eq. (J.1) in units of $2 \pi$.

The general expression of the relative weights $a_{\beta}=a_{\beta}\left(m_{\beta,+1}, m_{\beta,-1}\right)$ in Eq. (193), reads [51,63,64],

$$
a_{\beta}\left(m_{\beta,+1}, m_{\beta,-1}\right)=\left(\prod_{l= \pm} a_{\beta, l}\left(m_{\beta, l}\right)\right)(1+\mathcal{O}(\ln L / L)) \text { where } \beta=c, s 1
$$

where,

$$
a_{\beta, \iota}\left(m_{\beta, \iota}\right)=\prod_{j=1}^{m_{\beta, \iota}} \frac{\left(2 \Delta_{\beta}^{\iota}+j-1\right)}{j}=\frac{\Gamma\left(m_{\beta, \iota}+2 \Delta_{\beta}^{\iota}\right)}{\Gamma\left(m_{\beta, \iota}+1\right) \Gamma\left(2 \Delta_{\beta}^{\iota}\right)} \text { where } \beta=c, s 1 \text { and } \iota= \pm,
$$

and $\Gamma(x)$ is the usual gamma function. The relative weights, Eq. (J.3), are associated with the tower of excited energy eigenstates generated by the processes $(C)$ as defined in Section 7.2.

For $m_{\beta, l}=1$, Eq. (J.4) leads to,

$$
a_{\beta, \iota}(1)=2 \Delta_{\beta}^{\iota}=\left(\frac{\delta \bar{q}_{F \beta}^{\iota}}{(2 \pi / L)}\right)^{2} \text { where } \beta=c, s 1 \text { and } \iota= \pm .
$$

Here $a_{\beta, \iota}(1)$ is the relative weight of the $\alpha, \iota$ pseudofermion spectral function $m_{\beta, \iota}=1$ peaks. It can be written as given in Eq. (194). Moreover, $\delta \bar{q}_{F \beta}^{\iota} /(2 \pi / L)=\iota \delta N_{\beta, \iota}^{F}+\Phi_{\beta}\left(\iota q_{F \beta}\right)$ is the excited-state canonical momentum $\beta=c, s 1 ; \iota= \pm$ Fermipoint deviation. The $\beta=c, s 1$ weights $a_{\beta, \iota}(1)$ correspond to the particular cases $a_{\beta}(1,0)=2 \Delta_{\beta}^{+1}$ and $a_{\beta}(0,1)=2 \Delta_{\beta}^{-1}$ of the general relative weights, Eq. (J.3),

The $\delta$-functions in the pseudofermion spectral function expression, Eq. (193), impose the important equality $\left(\left(L / 4 \pi v_{\beta}\right)\right.$ $\left.\left(\omega^{\prime}+\iota v_{\beta} k^{\prime}\right)-\Delta_{\beta}^{\iota}\right)=m_{\beta, \imath}$. Hence $\left(\left(L / 4 \pi v_{\beta}\right)\left(\omega^{\prime}+\iota v_{\beta} k^{\prime}\right)-\Delta_{\beta}^{\iota}\right)$ is proportional to $L$. This implies that for any arbitrarily small $k^{\prime}$ and $\omega^{\prime}$ values for which $0<\left(\omega^{\prime}+\iota v k^{\prime}\right) /(4 \pi v) \ll 1$ the corresponding values of the $\iota= \pm$ integer numbers,

$$
\left.m_{\iota}=\frac{L}{4 \pi v_{\beta}}\left(\omega^{\prime}+\iota v_{\beta} k^{\prime}\right)-\Delta_{\beta}^{\iota}\right),
$$

are in the TL such that $m_{\beta, \iota} \gg 1$. Hence in the TL the $\beta, \iota$ relative weight, Eq. (J.4), has the following asymptotic behavior $[51,63,64]$,

$$
a_{\beta, \iota}\left(m_{\beta, \iota}\right) \approx \frac{1}{\Gamma\left(2 \Delta_{\beta}^{\iota}\right)}\left(m_{\beta, \iota}+\Delta_{\beta}^{\iota}\right)^{2 \Delta_{\beta}^{\iota}-1} \text { for } 2 \Delta_{\beta}^{\iota} \neq 0 \text { where } \beta=c, s 1 \text { and } \iota= \pm .
$$

Furthermore, in the TL the $\beta=c, s 1$ lowest peak weight $A_{\beta}^{(0,0)}$, Eq. (J.2), can be written as,

$$
A_{\beta}^{(0,0)}=\frac{F_{\beta}^{(0,0)}}{\left(L S_{\beta}\right)^{-1+2 \Delta_{\beta}^{+1}+2 \Delta_{\beta}^{-1}}} \text { where } \beta=c, s 1 .
$$

Here $F_{\beta}^{(0,0)}$ and $S_{\beta}$ are in the TL independent of $L$ and $2 \Delta_{c}^{+1}, 2 \Delta_{c}^{-1}, 2 \Delta_{s 1}^{+1}$, and $2 \Delta_{s 1}^{-1}$ are the four functionals, Eq. (J.5).

In the general case in which the values of such four functionals are finite, one finds from the use of Eq. (J.7) in the $\beta=c, s 1$ pseudofermion spectral function expression, Eq. (193), that in the TL it can be written as,

$$
B_{Q_{\beta}}\left(k^{\prime}, \omega^{\prime}\right)=\frac{L}{4 \pi v_{\beta}} A_{\beta}^{(0,0)} \prod_{\iota= \pm} a_{\beta, \iota}\left(\frac{\omega^{\prime}+\iota v_{\beta} k^{\prime}}{4 \pi v_{\beta} / L}\right) \text { where } \beta=c, s 1 .
$$


Further use in this expression of Eqs. (J.7) and (J.8) leads finally to the following expression for the $\beta=c, s 1$ pseudofermion spectral function, Eq. (193), valid in the TL,

$$
B_{Q_{\beta}}\left(k^{\prime}, \omega^{\prime}\right)=\frac{F_{\beta}^{(0,0)}}{4 \pi v_{\beta} S_{\beta}} \prod_{\imath= \pm} \frac{\Theta\left(\omega^{\prime}+\iota v_{\beta} k^{\prime}\right)}{\Gamma\left(2 \Delta_{\beta}^{\prime}\right)}\left(\frac{\omega^{\prime}+\iota v_{\beta} k^{\prime}}{4 \pi v_{\beta} S_{\beta}}\right)^{-1+2 \Delta_{\beta}^{\prime}} \text { where } \beta=c, s 1 .
$$

Here $F_{\beta}^{(0,0)}$ and $S_{\beta}$ are the $L$ independent quantities in the $A_{\beta}^{(0,0)}$ expression, Eq. (J.8). (The product $S_{c} \times S_{s 1}$ reads 1 both in the $u \rightarrow 0$ and $u \rightarrow \infty$ limits.)

In applications of the 1D Hubbard model PDT to the description of the ARPES of 1D metallic states in physical systems, the one-electron removal spectral function at zero spin density is that of interest. For electronic densities in the range $n_{e} \in[0,1]$, that spectral function has three main branch lines called $c, c^{\prime}$, and $s 1$ branch line. Their energy spectra have the following simple expressions in terms of the $\beta=c$ and $\beta=s 1$ bands energy dispersions $\varepsilon_{\beta}\left(q_{j}\right)$ defined in Eq. (152),

$$
\begin{aligned}
\omega_{c}(k) & =\varepsilon_{c}\left(|k|+k_{F}\right) \leq 0 \\
k & =-\operatorname{sgn}\{k\} k_{F}-q \in\left[-k_{F}, k_{F}\right] \\
\omega_{c^{\prime}}(k) & =\varepsilon_{c}\left(|k|-k_{F}\right) \leq 0 \\
k & =\operatorname{sgn}\{k\} k_{F}-q \in\left[-3 k_{F}, 3 k_{F}\right] . \\
\omega_{s 1}(k) & =\varepsilon_{S 1}(k)=\varepsilon_{S 1}(k) \leq 0 \\
k & =-q \in\left[-k_{F}, k_{F}\right] .
\end{aligned}
$$

Here $k$ is the one-electron excitation momentum.

The corresponding PDT momentum dependent exponents in Eq. (195) that control the line shape of the one-electron removal spectral function near the $c, c^{\prime}$, and $s 1$ branch lines are given by,

$$
\begin{aligned}
\zeta_{c}(k) & =-\frac{1}{2}+\sum_{\imath= \pm 1}\left(\frac{\xi_{0}}{4}-\Phi_{c c}\left(\iota 2 k_{F}, q\right)\right)^{2} \\
k & =\in\left[-k_{F}, k_{F}\right], \\
q & =-\operatorname{sgn}\{k\} k_{F}-k \in\left[-2 k_{F},-k_{F}\right] \text { and } \in\left[k_{F}, 2 k_{F}\right], \\
\zeta_{c^{\prime}}(k) & =-\frac{1}{2}+\sum_{\iota= \pm 1}\left(\frac{\xi_{0}}{4}-\Phi_{c c}\left(\iota 2 k_{F}, q\right)\right)^{2} \\
k & =\in\left[-3 k_{F}, 3 k_{F}\right], \\
q & =\operatorname{sgn}\{k\} k_{F}-k \in\left[-2 k_{F}, k_{F}\right] \text { and } \in\left[-k_{F}, 2 k_{F}\right] . \\
\zeta_{s 1}(k) & =-1+\sum_{\iota= \pm 1}\left(\frac{\iota}{2 \xi_{0}}+\Phi_{c s 1}\left(\iota 2 k_{F}, q^{\prime}\right)\right)^{2} \\
k & \in\left[-k_{F}, k_{F}\right] \text { and } q^{\prime}=-k \in\left[-k_{F}, k_{F}\right] .
\end{aligned}
$$

The parameter $\xi_{0}$ appearing here is given by $\xi_{0}=\xi_{0}\left(r_{c}^{0}\right)$ where the function $\xi_{0}(r)$ is the unique solution of the integral equation, Eq. (74) of Ref [92] with $x=r$. It has limiting values $\xi_{0}=\sqrt{2}$ for $u \rightarrow 0$ and $\xi_{0}=1$ for $u \rightarrow \infty$. The $c$ pseudofermion phase shifts $\Phi_{c c}\left( \pm 2 k_{F}, q\right)$ and $\Phi_{c s 1}\left( \pm 2 k_{F}, q^{\prime}\right)$ also appearing in the exponents expressions provided in Eq. (J.12) are through the general relation $\Phi_{\beta \beta^{\prime}}\left(q_{j}, q_{j^{\prime}}\right)=\bar{\Phi}_{\beta \beta^{\prime}}\left(r, r^{\prime}\right)$, Eq. (154), defined in terms of corresponding rapidity phase shifts $\bar{\Phi}_{c c}\left( \pm r_{c}^{0}, r\right)$ and $\bar{\Phi}_{c s 1}\left( \pm r_{c}^{0}, r^{\prime}\right)$, respectively. The latter are defined by the integral equations given in Appendix $H$.

\section{References}

[1] B. Sutherland, Beautiful Models: 70 Years of Exactly Solved Quantum Many-Body Problems, World Scientific, Singapore, 2004.

[2] J. Voit, Rep. Progr. Phys. 57 (1994) 977.

[3] H.J. Schulz, Phys. Rev. Lett. 64 (1990) 2831.

[4] K.-V. Pham, M. Gabay, P. Lederer, Phys. Rev. B 61 (2000) 16397.

[5] B.J. Kim, H. Koh, E. Rotenberg, S.-J. Oh, H. Eisaki, N. Motoyama, S. Uchida, T. Tohyama, S. Maekawa, Z.-X. Shen, C. Kim, Nat. Phys. 2 (2006) 397.

[6] R. Claessen, Nature 485 (2012) 46.

[7] J. Schlappa, K. Wohlfeld, K.J. Zhou, M. Mourigal, M.W. Haverkort, V.N. Strocov, L. Hozoi, C. Monney, S. Nishimoto, S. Singh, A. Revcolevschi, J.-S. Caux, L. Patthey, H.M. Rønnow, J. van den Brink, T. Schmitt, Nature 485 (2012) 82.

[8] V. Bisogni, K. Wohlfeld, S. Nishimoto, C. Monney, J. Trinckauf, K. Zhou, R. Kraus, K. Koepernik, C. Sekar, V. Strocov, B. Büchner, T. Schmitt, J. van den Brink, J. Geck, Phys. Rev. B 114 (2015) 096402.

[9] J.W. Allen, G.-H. Gweon, R. Claessen, K. Matho, J. Phys. Chem. Solids 56 (1995) 1849.

[10] F. Zwick, S. Brown, G. Margaritondo, C. Merlic, M. Onellion, J. Voit, M. Grioni, Phys. Rev. Lett. 79 (1997) 3982.

[11] J.W. Allen, Solid State Commun. 123 (2002) 469, Section 6.

[12] G.-H. Gweon, J.D. Denlingerb, C.G. Olsonc, H. Höchstd, J. Marcuse, C. Schlenkere, Physica B 312-313 (2002) 584.

[13] J.M.P. Carmelo, D. Bozi, K. Penc, J. Phys.: Condens. Matter 20 (2008) 415103. 
[14] R. Claessen, M. Sing, U. Schwingenschlögl, P. Blaha, M. Dressel, C.S. Jacobsen, Phys. Rev. Lett. 88 (2002) 096402.

[15] M. Sing, U. Schwingenschlögl, R. Claessen, P. Blaha, J.M.P. Carmelo, L.M. Martelo, P.D. Sacramento, M. Dressel, C.S. Jacobsen, Phys. Rev. B 68 (2003) 125111.

[16] J.M.P. Carmelo, K. Penc, L.M. Martelo, P.D. Sacramento, J.M.B. Lopes dos Santos, R. Claessen, M. Sing, U. Schwingenschlögl, Europhys. Lett. 67 (2004) 233.

[17] C. Kim, A.Y. Matsuura, Z.-X. Shen, N. Motoyama, H. Eisaki, S. Uchida, T. Tohyama, S. Maekawa, Phys. Rev. Lett. 77 (1996) 4054.

[18] C. Kim, Z.-X. Shen, N. Motoyama, H. Eisaki, S. Uchida, T. Tohyama, S. Maekawa, Phys. Rev. B 56 (1997) 15589.

[19] H. Fujisawa, T. Yokoya, T. Takahashi, S. Miyasaka, M. Kibune, H. Takagi, Phys. Rev. B 59 (1999) 7358.

[20] M.Z. Hasan, P.A. Montano, E.D. Isaacs, Z.-X. Shen, H. Eisaki, S.K. Sinha, Z. Islam, N. Motoyama, S. Uchida, Phys. Rev. Lett. 88 (2002) 177403.

[21] K. Kobayashi, T. Mizokawa, A. Fujimori, M. Isobe, Y. Ueda, Phys. Rev. Lett. 80 (1998) 3121.

[22] K. Kobayashi, T. Mizokawa, A. Fujimori, M. Isobe, Y. Ueda, T. Tohyama, S. Maekawa, Phys. Rev. Lett. 82 (1999) 803.

[23] S. Suga, A. Shigemoto, A. Sekiyama, S. Imada, A. Yamasaki, A. Irizawa, S. Kasai, Y. Saithoh, T. Muro, N. Tomita, K. Nasu, H. Eisaki, Y. Ueda, Phys. Rev. B 70 (2004) 155106.

[24] T.E. Kidd, T. Valla, P.D. Johnson, K.W. Kim, G.D. Gu, C.C. Homes, Phys. Rev. B 77 (2008) 054503.

[25] Y. Ohtsubo, J.-I. Kishib, K. Hagiwara, P. Le Fèvre, F. Bertran, A. Taleb-Ibrahimi, H. Yamane, S.-I. Ideta, M. Matsunami, K. Tanaka, S.-I. Kimura, Phys. Rev. Lett. 115 (2015) 256404.

[26] Y. Ma, H.C. Diaz, J. Avila, C. Chen, V. Kalappattil, R. Das, M.-H. Phan, T. Čadež, J.M.P. Carmelo, M.C. Asensio, M. Batzill, Nature Comm. 8 (2017) 14231.

[27] J. Fink, M. Knupfer, S. Atzkern, M.S. Golden, J. Elec. Spect. Rel. Phenom. 117 (2001) 287.

[28] X.-W. Guan, M.T. Batchelor, C. Lee, Rev. Modern Phys. 85 (2013) 1633.

[29] Y. Jianga, X.-W. Guan, J. Caoe, H.-Q. Lin, Nuclear Phys. B 895 (2015) 206.

[30] M.A. Cazalilla, R. Citro, T. Giamarchi, E. Orignac, M. Rigol, Rev. Modern Phys. 83 (2011) 1405.

[31] D.S. Petrov, G.V. Shlyapnikov, J.T.M. Walraven, Phys. Rev. Lett. 85 (2000) 3745.

[32] S. Murmann, F. Deuretzbacher, G. Zürn, J. Bjerlin, S.M. Reimann, L. Santos, T. Lompe, S. Jochim, Phys. Rev. Lett. 115 (2015) 215301.

[33] V.N. Golovach, A. Minguzzi, L.I. Glazman, Phys. Rev. A 80 (2009) 043611.

[34] N. Fabbri, M. Panfil, D. Clément, L. Fallani, M. Inguscio, C. Fort, J.-S. Caux, Phys. Rev. A 91 (2015) 043617.

[35] D. Jaksch, P. Zoller, Ann. Phys. 315 (2005) 52.

[36] F. Massel, V. Penna, Phys. Rev. A 72 (2005) 053619.

[37] M.-X. Huo, D.G. Angelakis, L.C. Kwek, New J. Phys. 14 (2012) 075027.

[38] M. Cheneau, P. Barmettler, D. Poletti, M. Endres, P. Schauss, T. Fukuhara, C. Gross, I. Bloch, C. Kollath, S. Kuhr, Nature 481 (2012) 484.

[39] Y. Jompol, C.J.B. Ford, J.P. Griffiths, I. Farrer, G.A.C. Jones, D. Anderson, D.A. Ritchie, T.W. Silk, A.J. Schofield, Science 325 (2009) 597.

[40] H. Bethe, Z. Phys. 71 (1931) 205.

[41] W. Heisenberg, Z. Phys. 49 (1928) 619.

[42] A. Luther, I. Peschel, Phys. Rev. B 9 (1974) 2911.

[43] A. Luther, I. Peschel, Phys. Rev. B 12 (1975) 3908.

[44] J. Sólyom, Adv. Phys. 28 (1979) 201.

[45] H.W. Blöte, J.L. Cardy, M.P. Nightingale, Phys. Rev. Lett. 56 (1985) 742.

[46] I. Affleck, Phys. Rev. Lett. 56 (1985) 746.

[47] S. Tomonaga, Progr. Theoret. Phys. 5 (1950) 544.

[48] J.M. Luttinger, J. Math. Phys. 4 (1963) 1154.

[49] F.A. Smirnov, Form Factors in Completely Integrable Models of Quantum Field Theory, in: Advanced Series in Mathematical Physics, vol. 14, World Scientific, Singapore, 1992.

[50] M. Jimbo, T. Miwa, Algebraic Analysis of Solvable Lattice Models, American Mathematical Society, Providence, 1994.

[51] J.M.P. Carmelo, K. Penc, D. Bozi, Nuclear Phys. B 725 (2005) 421; Nuclear Phys. B 737 (2006) 351 (erratum).

[52] A. Imambekov, L.I. Glazman, Science 323 (2009) 228.

[53] A. Imambekov, T.L. Schmidt, L.I. Glazman, Rev. Modern Phys. 84 (2012) 1253.

[54] F.H.L. Essler, Phys. Rev. B 81 (2010) 205120.

[55] L. Seabra, F.H.L. Essler, F. Pollmann, I. Schneider, T. Veness, Phys. Rev. B 90 (2014) 245127.

[56] R.G. Pereira, K. Penc, S.R. White, P.D. Sacramento, J.M.P. Carmelo, Phys. Rev. B 85 (2012) 165132.

[57] A. Imambekov, L.I. Glazman, Phys. Rev. Lett. 100 (2008) 206805.

[58] J.M.P. Carmelo, K. Penc, P.D. Sacramento, M. Sing, R. Claessen, J. Phys.: Condens. Matter 18 (2006) 5191.

[59] D. Bozi, J.M.P. Carmelo, K. Penc, P.D. Sacramento, J. Phys.: Condens. Matter 20 (2008) 022205.

[60] A. Moreno, A. Muramatsu, J.M.P. Carmelo, Phys. Rev. B 87 (2013) 075101.

[61] J.M.P. Carmelo, P.D. Sacramento, Ann. Phys. 369 (2016) 102.

[62] J.M.P. Carmelo, P.D. Sacramento, J.D.P. Machado, D.K. Campbell, J. Phys.: Condens. Matter. 27 (2015) 406001.

[63] J.M.P. Carmelo, T. Čadež, Nuclear Phys. B 904 (2016) 39.

[64] J.M.P. Carmelo, T. Čadež, Nuclear Phys. B 914 (2017) 461.

[65] N. Andrei, in: S. Lundquist, G. Morandi, Yu Lu (Eds.), Integrable Models in Condensed Matter Physics, in: Series on Modern Condensed Matter Physics, vol. 6, p. 458, World Scientific, Lecture Notes of ICTP Summer Course, cond-mat/9408101.

[66] F.H.L. Essler, V.E. Korepin, Phys. Rev. Lett. 72 (1994) 908.

[67] F.H.L. Essler, V.E. Korepin, Nuclear Phys. B 426 (1994) 505, Section 5.

[68] J. des Cloizeaux, J.J. Pearson, Phys. Rev. 128 (1962) 2131.

[69] E.H. Lieb, F.Y. Wu, Phys. Rev. Lett. 20 (1968) 1445; Phys. Rev. Lett. 21 (1968) 192 (erratum).

[70] E.H. Lieb, F.Y. Wu, Physica A 321 (2003) 1.

[71] A.A. Ovchinnikov, Sov. Phys. JETP-USSR 30 (1970) 1160.

[72] M. Takahashi, Progr. Theoret. Phys. 46 (1971) 401.

[73] M. Takahashi, Progr. Theoret. Phys. 47 (1972) 69.

[74] C.F. Coll, Phys. Rev. B 9 (1974) 2150.

[75] M. Fowler, M.W. Puga, Phys. Rev. B 18 (1978) 421.

[76] N. Andrei, J. Lowenstein, Phys. Rev. Lett. 43 (1979) 1698.

[77] N. Andrei, J. Lowenstein, Phys. Lett. 91B (1980) 401.

[78] L.D. Faddeev, L.A. Takhtajan, Phys. Lett. 85A (1981) 375.

[79] P.W. Anderson, Science 235 (1987) 1196.

[80] N.F. Mott, Rev. Modern Phys. 40 (1968) 677.

[81] L.C. Bartel, H.S. Jarret, Phys. Rev. B 10 (1974) 946. 
[82] J. Marbach, F.X. Bronold, H. Fehske, Phys. Rev. B 86 (2012) 115417.

[83] V.E. Korepin, Theoret. Math. Phys. 41 (1979) 953.

[84] V.E. Korepin, Comm. Math. Phys. 76 (1980) 165.

[85] A.H. Castro Neto, H.Q. Lin, Y.-H. Chen, J.M.P. Carmelo, Phys, Rev. B 50 (1994) 14032.

[86] J.M.P. Carmelo, H.Q. Lin, Modern Phys. Lett. B 7 (1993) 1387.

[87] J.M.P. Carmelo, T. Prosen, D.K. Campbell, Phys. Rev. B 92 (2015) 165133.

[88] J.M.P. Carmelo, T. Prosen, Nuclear Phys. B 92 (2017) 62.

[89] J.M.P. Carmelo, P. Horsch, P.A. Bares, A.A. Ovchinnikov, Phys. Rev. B 44 (1991) 9967.

[90] J. Carmelo, A.A. Ovchinnikov, J. Phys.: Condens. Matter. 3 (1991) 757.

[91] J.M.P. Carmelo, P. Horsch, Phys. Rev. Lett. 68 (1992) 871.

[92] J.M.P. Carmelo, P. Horsch, A.A. Ovchinnikov, Phys. Rev. B 45 (1992) 7899.

[93] J.M.P. Carmelo, P. Horsch, A.A. Ovchinnikov, Phys. Rev. B 46 (1992) 14728.

[94] J.M.P. Carmelo, A.H. Castro Neto, Phys. Rev. Lett. 70 (1993) 1904; 74 (1995) 3089 (erratum).

[95] J.M.P. Carmelo, P. Horsch, D.K. Campbell, A.H. Castro Neto, Phys. Rev. B (RC) 48 (1993) 4200

[96] J.M.P. Carmelo, A.H. Castro Neto, D.K. Campbell, Phys. Rev. B 50 (1994) 3667.

[97] J.M.P. Carmelo, A.H. Castro Neto, D.K. Campbell, Phys. Rev. B 50 (1994) 3683.

[98] J.M.P. Carmelo, N.M.R. Peres, D.K. Campbell, A.W. Sandvik, Z. Phys. B 103 (1997) 217.

[99] N.M.R. Peres, J.M.P. Carmelo, Phys. Rev. B 56 (1997) 3717.

[100] J.M.P. Carmelo, F. Guinea, P.D. Sacramento, Phys. Rev. B 55 (1997) 7565.

[101] J.M.P. Carmelo, J.M.E. Guerra, J.M.B. Lopes dos Santos, A.H. Castro Neto, Phys. Rev. Lett. 83 (1999) 3892.

[102] J.M.P. Carmelo, N.M.R. Peres, P.D. Sacramento, Phys. Rev. Lett. 84 (2000) 4673.

[103] N.M.R. Peres, R.G. Dias, P.D. Sacramento, J.M.P. Carmelo, Phys. Rev. B 61 (2000) 5169

[104] J.M.P. Carmelo, P.D. Sacramento, Phys. Rev. B 68 (2003) 085104.

[105] J.M.P. Carmelo, J.M. Román, K. Penc, Nuclear Phys. B 683 (2004) 387.

[106] E.H. Lieb, W. Liniger, Phys. Rev. 130 (1963) 1605.

[107] E.H. Lieb, Phys. Rev. 130 (1963) 1616.

[108] F.A. Berezin, G.P. Pokhil, V.M. Finkelberg, Vestn. Mosk. Uni. 1 (1964) 21.

[109] J.B. McGuire, J. Math. Phys. 5 (1964) 622.

[110] C.N. Yang, C.P. Yang, J. Math. Phys. 10 (1969) 1115.

[111] H.B. Thacker, Rev. Modern Phys. 53 (1981) 253.

[112] V.E. Korepin, N.M. Bogoliubov, A.G. Izergin, Quantum Inverse Scattering Method and Correlation Functions, Cambridge University Press, London, 1993.

[113] E.J.K.P. Nandani, R.A. Römer, S. Tan, X.-W. Guan, New J. Phys. 18 (2016) 055014

[114] G. Müller, H. Thomas, H. Beck, J.C. Bonner, Phys. Rev. B 24 (1981) 1429.

[115] M. Takahashi M, Thermodynamics of One-Dimensional Solvable Models, Cambridge University Press, London, 1999.

[116] M.C. Gutzwiller, Phys. Rev. Lett. 10 (1963) 159.

[117] J. Hubbard, Proc. R. Soc. Lond. Ser. A Math. Phys. Eng. Sci. 276 (1963) 238.

[118] F. Woynarovich, J. Phys. C: Solid State Phys. 15 (1982) 85.

[119] F. Woynarovich, J. Phys. C: Solid State Phys. 15 (1982) 97

[120] B.S. Shastry, Phys. Rev. Lett. 56 (1986) 1529; Phys. Rev. Lett. 56 (1986) 2334 (erratum).

[121] B.S. Shastry, Phys. Rev. Lett. 56 (1986) 2453.

[122] B.S. Shastry, J. Stat. Phys. 50 (1988) 57.

[123] E. Olmedilla, M. Wadati, Y. Akutsu, J. Phys. Soc. Japan 56 (1987) 2298.

[124] E. Olmedilla, M. Wadati, Phys. Rev. Lett. 60 (1988) 1595.

[125] M. Wadati, E. Olmedilla, Y. Akutsu, J. Phys. Soc. Japan 56 (1987) 1340.

[126] M. Shiroishi, M. Wadati, J. Phys. Soc. Japan 64 (1995) 57.

[127] P.B. Ramos, M.J. Martins, J. Phys. A: Math. Gen. 30 (1997) L195.

[128] M.J. Martins, P.B. Ramos, Nuclear Phys. B 522 (1998) 413.

[129] R. Yue, T. Deguchi, J. Phys. A 30 (1997) 849.

[130] F.H.L. Essler, H. Frahm, F. Göhmann, A. Klümper, V.E. Korepin, The One-Dimensional Hubbard Model, Cambridge University Press, Cambridge, UK, 2005, Section 7.2

[131] R. Hagemans, J.-S. Caux, J. Phys. A 40 (2007) 14605

[132] T. Deguchi, F.H.L. Essler, F. Göhmann, A. Klümper, V.E. Korepin, K. Kusakabe, Phys. Rep. 331 (2000) 197

[133] J. Sirker, R.G. Pereira, I. Affleck, Phys. Rev. B 83 (2011) 035115.

[134] S. Glawion, J. Heidler, M.W. Haverkort, L.C. Duda, T. Schmitt, V.N. Strocov, C. Monney, K.J. Zhou, A. Ruff, M. Sing, R. Claessen, Phys. Rev. Lett. 107 (2011) 107402

[135] S.E. Nagler, D.A. Tennant, R.A. Cowley, T.G. Perring, S.K. Satija, Phys. Rev. B 44 (1991) 12361.

[136] M.B. Stone, D.H. Reich, C. Broholm, K. Lefmann, C. Rischel, C.P. Landee, M.M. Turnbull, Phys. Rev. Lett. 91 (2003) 037205.

[137] I.A. Zaliznyak, H. Woo, T.G. Perring, C.L. Broholm, C.D. Frost, H. Takagi, Phys. Rev. Lett. 93 (2004) 087202.

[138] B. Lake, D.A. Tennant, C.D. Frost, S.E. Nagler, Nature Mater. 4 (2005) 329.

[139] M. Enderle, C. Mukherjee, B. Fåk, R.K. Kremer, J.-M. Broto, H. Rosner, S.-L. Drechsler, J. Richter, J. Malek, A. Prokofiev, W. Assmus, S. Pujol, J.-L. Raggazzoni1, H. Rakoto5, M. Rheinstädter, H.M. Rønnow, Europhys. Lett. 70 (2005) 237.

[140] G.J. Nilsen, H.M. Rønnow, A.M. Läuchli, F.P.A. Fabbiani, J. Sanchez-Benitez, K.V. Kamenev, A. Harrison, Chem. Mater. 20 (2008) 8.

[141] A.C. Walters, T.G. Perring, J.-S. Caux, A.T. Savici, G.D. Gu, C.-C. Lee, W. Ku, I.A. Zaliznyak, Nat. Phys. 5 (2009) 867.

[142] L. Braicovich, M. Moretti Sala, L.J.P. Ament, V. Bisogni, M. Minola, G. Balestrino, D. Di Castro, G.M. De Luca, M. Salluzzo, G. Ghiringhelli, J. van den Brink, Phys. Rev. B 81 (2010) 174533.

[143] O.J. Heilmann, E.H. Lieb, Ann. New York Acad. Sci. 172 (1971) 583.

[144] C.N. Yang, Phys. Rev. Lett. 63 (1989) 2144.

[145] C.N. Yang, S.C. Zhang, Modern Phys. Lett. B 4 (1990) 759.

[146] E.H. Lieb, Phys. Rev. Lett. 62 (1989) 1201.

[147] J.M.P. Carmelo, S. Östlund, M.J. Sampaio, Ann. Phys. 325 (2010) 1550.

[148] D. Baeriswyl, J. Carmelo, K. Maki, Synth. Met. 21 (1987) 271.

[149] C.N. Yang, Phys. Rev. Lett. 19 (1967) 1312.

[150] M. Ogata, H. Shiba, Phys. Rev. B 41 (1990) 2326. 
[151] M. Ogata, T. Sugiyama, H. Shiba, Phys. Rev. B 43 (1991) 8401.

[152] A. Parola, S. Sorella, Phys. Rev. Lett. 64 (1990) 1831.

[153] A. Parola, S. Sorella, Phys. Rev. Lett. 45 (1992) 13156

[154] Z.Y. Weng, D.N. Sheng, C.S. Ting, Z.B. Su, Phys. Rev. B 45 (1992) 7850.

[155] Z.Y. Weng, Phys. Rev. B 50 (1994) 13837.

[156] K. Penc, F. Mila, H. Shiba, Phys. Rev. Lett. 75 (1995) 894.

[157] K. Penc, K. Hallberg, F. Mila, H. Shiba, Phys. Rev. Lett. 77 (1996) 1390.

[158] K. Penc, K. Hallberg, F. Mila, H. Shiba, Phys. Rev. B 55 (1997) 15475.

[159] Y.R. Wang, Phys. Rev. B 51 (1995) 234.

[160] F.B. Gallagher, S. Mazumdar, Phys. Rev. B 56 (1997) 15025.

[161] F. Gebhard, K. Bott, M. Scheidler, P. Thomas, S.W. Koch, Phil. Mag. B 75 (1997) 47.

[162] K.A. Sidorova, S.G. Ovchinnikova, N.V. Tikhonov, J. Exp. Theor. Phys. 116 (2013) 330.

[163] D. Poilblanc, A. Feiguin, M. Troyer, E. Ardonne, P. Bonderson, Phys. Rev. B 87 (2013) 085106.

[164] M. Kormos, G. Mussardo, A. Trombettoni, Phys. Rev. A 81 (2010) 043606.

[165] B. Pozsgay, J. Stat. Mech. Theory Exp. (2011) P01011.

[166] B. Bertini, L. Piroli, P. Calabrese, J. Stat. Mech. Theory Exp. (2016) 063102.

[167] A.H. van Amerongen, J.J.P. van Es, P. Wicke, K.V. Kheruntsyan, N.J. van Druten, Phys. Rev. Lett. 100 (2008) 090402.

[168] N. Fabbri, D. Clément, L. Fallani, C. Fort, M. Inguscio, Phys. Rev. A 83 (2011) 031604.

[169] F. Meinert, M. Panfil, M.J. Mark, K. Lauber, J.-S. Caux, H.-C. Nägerl, Prob. Phys. Rev. Lett. 115 (2015) 085301.

[170] B. Fang, A. Johnson, T. Roscilde, I. Bouchoule, Phys. Rev. Lett. 116 (2016) 050402.

[171] L. Tonks, Phys. Rev. 50 (1936) 955.

[172] M. Girardeau, J. Math. Phys. 1 (1960) 516

[173] L. Hulthén, Ark. Mat. Astron. Fys. 26 (1938) 1

[174] N.M. Bogoliubov, A.G. Izergin, V.E. Korepin, Nuclear Phys. B 275 (1986) 687.

[175] N.M. Bogoliubov, A.G. Izergin, N.Y. Reshetikin, J. Phys. A 20 (1987) 5361.

[176] F. Woynarovich, H.P. Eckle, T.T. Truong, J. Phys. A 22 (1989) 4027.

[177] B.S. Shastry, Bill Sutherland, Phys. Rev. Lett. 65 (1990) 243.

[178] H. Nyquist, Phys. Rev. 32 (1928) 110.

[179] H.B. Callen, T.A. Welton, Phys. Rev. 83 (1951) 34.

[180] S. Mukerjee, B.S. Shastry, Phys. Rev. 77 (2008) 245131.

[181] J.M.P. Carmelo, L.M. Martelo, K. Penc, Nuclear Phys. B 737 (2006) 237.

[182] P.W. Anderson, Phys. Rev. Lett. 18 (1967) 1049.

[183] J.-S. Caux, P. Calabrese, Phys. Rev. A 74 (2006) 031605.

[184] N. Motoyama, H. Eisaki, S. Uchida, Phys. Rev. Lett. 76 (1996) 3212.

[185] K.R. Thurber, A.W. Hunt, T. Imai, F.C. Chou, Phys. Rev. Lett. 87 (2001) 247202.

[186] P.W. Anderson, Phys. Rev. 86 (1952) 694.

[187] E. Manousakis, Rev. Modern Phys. 63 (1991) 1.

[188] R.B. Griffiths, Phys. Rev. 133 (1964) A768.

[189] C.N. Yang, C.P. Yang, Phys. Rev. 150 (1966) 321.

[190] C.N. Yang, C.P. Yang, Phys. Rev. 150 (1966) 327.

[191] C.N. Yang, C.P. Yang, Phys. Rev. 151 (1966) 258.

[192] M. Gaudin, Phys. Rev. Lett. 26 (1971) 1301.

[193] M. Karbach, G. Müller, A.H. Bougourzi, A. Fledderjohann, K.H. Mütter, Phys. Rev. B 55 (1997) 12510.

[194] A. Abada, A.H. Bougourzi, B. Si-Lakhal, Nuclear Phys. B 497 (1997) 733.

[195] A.H. Bougourzi, M. Couture, M. Kacir, Phys. Rev. B 54 (1996) R12669.

[196] J.-.S. Caux, R. Hagemans, J. Stat. Mech. (2006) P12013.

[197] A. Imambekov, L.I. Glazman, Phys. Rev. Lett. 102 (2009) 126405.

[198] J.-.S. Caux, H. Konno, M. Sorrell, R. Weston, Phys. Rev. Lett. 106 (2011) 217203.

[199] A. Klauser, J. Mossel, J.-S. Caux, J. Brink, Phys. Rev. Lett. 106 (2011) 157205.

[200] X. Zotos, Phys. Rev. Lett. 82 (1999) 1764.

[201] T. Prosen, Phys. Rev. Lett. 106 (2011) 217206.

[202] T. Prosen, E. Ilievski, Phys. Rev. Lett. 111 (2013) 057203.

[203] T. Prosen, Phys. Rev. E 89 (2014) 012142.

[204] E. Ilievski, J. De Nardis, Phys. Rev. Lett. 119 (2017) 020602.

[205] M. Medenjak, C. Karrasch, T. Prosen, Phys. Rev. Lett. 119 (2017) 080602.

[206] F. He, Y.-Z. Jiang, Y.-C. Yu, H.-Q. Lin, X.-W. Guan, Phys. Rev. B 96 (2017) 220401.

[207] L.P. Kadanoff, A.C. Brown, Ann. Phys. NY 121 (1979) 318.

[208] A.A. Belavin, A.M. Polyakov, A.B. Zamolodchikov, Nuclear Phys. B 241 (1984) 333.

[209] P. Di Francesco, P. Mathieu. D. Sénéchal, Conformal Field Theory, Springer, Berlin, 1997 and references therein.

[210] I. Affleck, Les Houches, Session XLIX, Fields, Strings and Critical Phenomena, 1989.

[211] A.O. Gogolin, A.A. Nersesyan, A.M. Tsvelik, Bosonization and Strongly Correlated Systems, Cambridge University Press, Cambridge, 1998.

[212] I. Affleck, J. Phys. A: Math. Gen. 31 (1998) 4573.

[213] S. Lukyanov, Phys. Rev. B 59 (1999) 11163.

[214] S. Lukyanov, V. Terras, Nuclear Phys. B 654 (2003) 323.

[215] K. Lefmann, C. Rischel, Phys. Rev. B 54 (1996) 6340.

[216] M. Karbach, G. Müller, Phys. Rev. B 62 (2000) 14871.

[217] M. Karbach, D. Biegel, G. Müller, Phys. Rev. B 66 (2002) 054405.

[218] D. Biegel, M. Karbach, G. Müller, Europhys. Lett. 59 (2002) 882.

[219] N. Kitanine, J.M. Maillet, V. Terras, Nuclear Phys. B 554 (1999) 647.

[220] N. Kitanine, J.M. Maillet, V. Terras, Nuclear Phys. B 567 (2000) 554.

[221] J.-S. Caux, J.M. Maillet, Phys. Rev. Lett. 95 (2005) 077201.

[222] R.G. Pereira, S.R. White, I. Affleck, Phys. Rev. Lett. 100 (2008) 027206.

[223] M. Kohno, Phys. Rev. Lett. 102 (2009) 037203.

[224] H. Frahm, V.E. Korepin, Phys. Rev. B 42 (1990) 10553. 
[225] H. Frahm, V.E. Korepin, Phys. Rev. B 43 (1991) 5653.

[226] K. Penc, F. Woynarovich, Z. Phys. B 85 (1991) 269.

[227] H. Shiba, Phys. Rev. B 6 (1972) 930.

[228] M. Takahashi, Progr. Theoret. Phys. 42 (1969) 1098.

[229] A. Klümper, A. Schadschneider, J. Zittartz, Z. Phys. B 78 (1990) 99

[230] F. Woynarovich, J. Phys. C 16 (1983) 5293.

[231] F. Woynarovich, J. Phys. C 16 (1983) 6593.

[232] N. Kawakami, T. Usuki, A. Okiji, Phys. Lett. A 137 (1989) 287.

[233] T. Usuki, N. Kawakami, A. Okiji, J. Phys. Soc. Japan 59 (1990) 1357.

[234] F. Woynarovich, H.P. Eckle, J. Phys. A 20 (1987) L443.

[235] F. Woynarovich, J. Phys. A 22 (1989) 4243.

[236] D. Controzzi, F.H.L. Essler, Phys. Rev. B 66 (2002) 165112.

[237] F.H.L. Essler, A.M. Tsvelik, Phys. Rev. B 65 (2002) 115117.

[238] F.H.L. Essler, A., M. Tsvelik, Phys. Rev. Lett. 90 (2003) 126401.

[239] E. Jeckelmann, F. Gebhard, F.H.L. Essler, Phys. Rev. Lett. 85 (2000) 3910.

[240] E. Melzer, Nuclear Phys. B 443 (1995) 553.

[241] F. Woynarovich, P. Forgacs, Nuclear Phys. B 498 (1997) 565.

[242] F. Woynarovich, P. Forgacs, Nuclear Phys. B 538 (1999) 701.

[243] F.H.L. Essler, V.E. Korepin, K. Schoutens, Phys. Rev. Lett. 67 (1991) 3848.

[244] F.H.L. Essler, V.E. Korepin, K. Schoutens, Nuclear Phys. B 372 (1992) 559.

[245] F.H.L. Essler, V.E. Korepin, K. Schoutens, Nuclear Phys. B 384 (1992) 431.

[246] G. Jüttner, A. Klümper, J. Suzuki, Nuclear Phys. B 522 (1998) 471.

[247] K. Tsunetsugu, J. Phys. Soc. Japan 60 (1991) 1460.

[248] Y. Umeno, M. Shiroishi, A. Klümper, Europhys. Lett. 62 (2003) 384.

[249] D.B. Uglov, V.E. Korepin, Phys. Lett. A 190 (1994) 238.

[250] S. Murakami, F. Göhmann, Phys. Lett. A 227 (1997) 216

[251] S. Murakami, F. Göhmann, Nuclear Phys. B 512 (1998) 637.

[252] S. Östlund, E. Mele, Phys. Rev. B 44 (1991) 12413.

[253] D. Braak, N. Andrei, Nuclear Phys. B 542 (1999) 551.

[254] J. Stein, J. Stat. Phys. 88 (1997) 487.

[255] A.L. Chernyshev, D. Galanakis, P. Phillips, A.V. Rozhkov, A.-M.S. Tremblay, Phys. Rev. B 70 (2004) 235111.

[256] J.-Y.P. Delannoy, M.J.P. Gingras, P.C. Holdsworth, A.-M.S. Tremblay, Phys. Rev. B 79 (2009) 235130.

[257] A.H. MacDonald, S.M. Girvin, D. Yoshioka, Phys. Rev. B 37 (1988) 9753.

[258] A.H. MacDonald, S.M. Girvin, D. Yoshioka, Phys. Rev. B 41 (1990) 2565.

[259] A.B. Harris, R.V. Lange, Phys. Rev. 157 (1967) 295.

[260] S. Östlund, M. Granath, Phys. Rev. Lett. 96 (2006) 066404.

[261] H.V. Kruis, I.P. McCulloch, Z. Nussinov, J. Zaanen, Phys. Rev. B 70 (2004) 075109.

[262] F.D.M. Haldane, Phys. Rev. Lett. 67 (1991) 937.

[263] S.-J. Gu, N.M.R. Peres, J.M.P. Carmelo, J. Phys.: Condens. Matter 19 (2007) 506203.

[264] J.M.P. Carmelo, S. Nemati, T. Prosen, Nuclear Phys. B 930 (2018) 418.

[265] D. Larsson, H. Johannesson, Phys. Rev. Lett. 95 (2005) 196406; Phys. Rev. Lett. 96 (2006) 169906 (erratum).

[266] S.-J. Gu, S.-S. Deng, Y.-Q. Li, H.-Q. Lin, Phys. Rev. Lett. 93 (2004) 086402.

[267] E. Ilievski, J. De Nardis, Phys. Rev. B 96 (2017) 081118(R).

[268] J.M.P. Carmelo, J. Phys.: Condens. Matter 17 (2005) 5517.

[269] H. Benthien, F. Gebhard, E. Jeckelmann, Phys. Rev. Lett. 92 (2004) 256401.

[270] F.H.L. Essler, V.E. Korepin, Phys. Rev. B 59 (1999) 1734.

[271] M.J. Bhaseen, F.H.L. Essler, A. Grage, Phys. Rev. B 71 (2005) 020405(R).

[272] C. Blumenstein, J. Schäfer, S. Mietke, S. Meyer, A. Dollinger, M. Lochner, X.Y. Cui, L. Patthey, R. Matzdorf, R. Claessen, Nat. Phys. 7 (2011) 776 and Supplementary Information.

[273] V.V. Flambaum, G.F. Gribakin, C. Harabati, Phys. Rev. A 59 (1999) 1998.

[274] J.M.P. Carmelo, T. Prosen, D.K. Campbell, Phys. Rev. B 63 (2001) 205114.

[275] A. Damascelli, Z. Hussain, Z.-X. Shen, Rev. Modern Phys. 75 (2003) 473.

[276] V.Z. Kresin, Y.N. Ovchinnikov, S.A. Wolf, Phys. Rep. 431 (2006) 231.

[277] P.A. Lee, N. Nagaosa, X.-G. Wen, Rev. Modern Phys. 78 (2006) 17.

[278] S. Hüfner, M.A. Hussain, A. Damascelli, G.A. Sawatzky, Rep. Progr. Phys. 71 (2008) 062501.

[279] D.J. Scalapino, Rev. Modern Phys. 84 (2012) 1383.

[280] P. Phillips, Rev. Modern Phys. 82 (2010) 1719.

[281] J.M.P. Carmelo, M.A.N. Araújo, S.R. White, M.J. Sampaio, Phys. Rev. B 86 (2012) 064520.

[282] Y.R. Wang, Phys. Rev. B 46 (1992) 151. 